Supporting Information for:

\title{
Two Complementary Routes to 7-Substituted Chlorins. Partial Mimics of Chlorophyll $b$
}

\author{
Chinnasamy Muthiah, Marcin Ptaszek, Tien M. Nguyen, Kyle M. Flack \\ and Jonathan S. Lindsey
}

Table of Contents

\begin{tabular}{|c|c|c|}
\hline I. & Exploratory Work & S1-S2 \\
\hline II. & Streamlined Synthesis of Chlorin $\mathbf{Z n C}-\mathbf{T}^{\mathbf{5}}$ & $\mathrm{S} 2$ \\
\hline III. & ${ }^{1} \mathrm{H}$ NMR Spectra of Selected Bromochlorins & S3 \\
\hline IV. & Experimental Section & S3-S5 \\
\hline $\mathrm{V}$. & X-ray Structural Data for 7-Bromochlorin $\mathbf{Z n C}-\mathbf{B r}^{7^{7}} \mathbf{T}^{\mathbf{1 0}}$ & S6-S13 \\
\hline VI. & References & $\mathrm{S} 13$ \\
\hline VII & Spectral Data & S13-S11 \\
\hline
\end{tabular}

\section{Exploratory Work}

During the course of exploring new routes to 7 -substituted chlorins, we prepared several novel dipyrromethanes. The syntheses of the dipyrromethanes are reported here.

The dithiolane group has been extensively used as a potent formyl synthon in organic synthesis. ${ }^{\mathrm{S} 1}$ The route to an Eastern half bearing a dithiolane unit begins with protection of the formyl group of pyrrole-2-carboxaldehyde (Scheme S2). Thus, 2-(1,3-dithiolane-2-yl)pyrrole ${ }^{4}$ was readily prepared from pyrrole-2-carboxaldehyde. Vilsmeier formylation of 2-(1,3dithiolane-2-yl)pyrrole afforded the mono-protected 2,5-diformyl product $\mathbf{S 1}^{4}$ in $55 \%$ yield. Treatment of $\mathbf{S 1}$ with 1 molar equivalent of NBS at $-78{ }^{\circ} \mathrm{C}$ gave the 4-bromo derivative $\mathbf{S 2}$ in $51 \%$ yield. Reduction of $\mathbf{S 2}$ with $\mathrm{LiBH}_{4}$ at $0{ }^{\circ} \mathrm{C}$ afforded the corresponding carbinol derivative. Treatment of the carbinol with an excess amount of pyrrole under acidic conditions afforded the $\beta$-substituted, mono-protected dipyrromethane $\mathbf{S 3}$ in $61 \%$ yield. Deprotection of the 1,3dithiolane group has been achieved under numerous conditions: ${ }^{\mathrm{S} 2 \mathrm{~S} 5}$ (1) $\mathrm{BF}_{3} \cdot \mathrm{Et}_{2} \mathrm{O}$ and $\mathrm{HgO}$ in THF or aqueous THF was used to deprotect the dithiolane group in $\beta$-alkyl substituted dipyrromethanes; (2) $\mathrm{HgCl}_{2}$ and $\mathrm{CaCO}_{3}$ in $\mathrm{CH}_{3} \mathrm{CN}$ or aqueous $\mathrm{CH}_{3} \mathrm{CN}$ was used to deprotect the dithiolane group both in pyrrole and in non-pyrrolic derivatives; (3) NBS in aqueous acetone was used to deprotect the dithiolane group in pyrrole derivatives; and (4) Selectfluor ${ }^{\mathrm{TM}}$ was also known to cleave the dithiolane group. Attempted deprotection of the 1,3-dithiolane group in $\mathbf{S 3}$ to the corresponding formydipyrromethane using the above reaction conditions was unsuccessful.

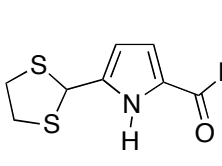

S1

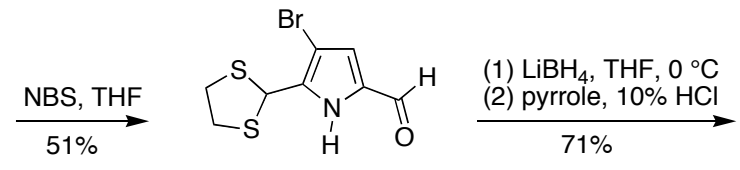

S2

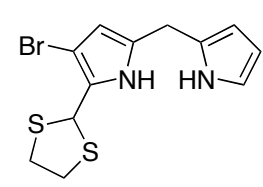

S3

The synthesis of a $\beta$-bromo substituted dipyrromethane is shown in Scheme S3. Reduction of $\mathbf{S 4}^{\mathrm{S} 6}$ with $\mathrm{LiBH}_{4}$ at $0{ }^{\circ} \mathrm{C}$ gave the corresponding pyrrole-carbinol derivative. Treatment of the latter with an excess amount of pyrrole under acidic conditions afforded the $\beta$ - 
bromo substituted dipyrromethane $\mathbf{S 5}$ in 58\% yield. Attempts to brominate 2bromodipyromethane $\mathbf{S 5}$ at the 9-position ( $\alpha$-position of the pyrrole lacking a bromine atom) were unsuccessful.

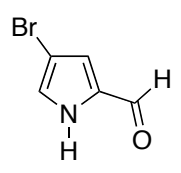

S4

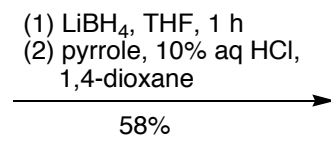

(1) $\mathrm{LiBH}_{4}, \mathrm{THF}, 1 \mathrm{~h}$
(2) pyrrole, $10 \%$ aq $\mathrm{HCl}$,
1,4-dioxane

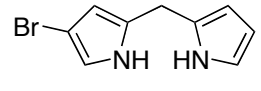

S5

The synthesis of a dipyrromethane bearing a carbomethoxy group is shown in Scheme S4. Treatment of 4-carbomethoxypyrrole-2-carboxaldehyde (S6) ${ }^{\mathrm{S} 7}$ with $p$-tolylmagnesium bromide gave the pyrrole-carbinol S7. Treatment of the latter with 2-decylthiopyrrole in he presence of $\mathrm{InCl}_{3}$ gave dipyrromethane $\mathbf{S 8}$ in $62 \%$ yield. Attempts to use $\mathbf{S 8}$ in chlorin syntheses gave poor results.

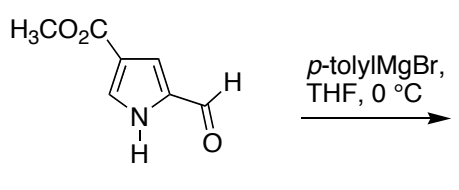

S6
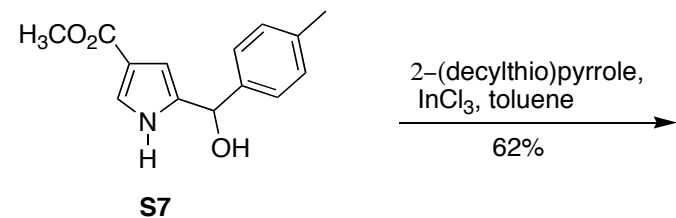

S7

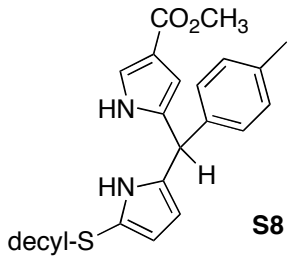

\section{Streamlined Synthesis of Chlorin ${\mathrm{ZnC}-\mathrm{T}^{5}}^{5}$}

The synthesis of chlorin $\mathbf{Z n C - \mathbf { T } ^ { \mathbf { 5 } }}$ was carried out in a streamlined fashion as shown in Scheme S1. Thus, 1-(4-methylbenzoyl)dipyrromethane (S9) ${ }^{10}$ was treated with NBS in THF solution at $-78{ }^{\circ} \mathrm{C}$ to give the 9-bromo derivative. After $1 \mathrm{~h}$ the reaction mixture was brought to room temperature, diluted with THF and methanol, and treated with excess $\mathrm{NaBH}_{4}$ to give the 9bromodipyrromethane-1-carbinol (Eastern half). Then the reaction mixture was quenched by the addition of aqueous $\mathrm{NH}_{4} \mathrm{Cl}$ and extracted with diethyl ether. The organic extract was diluted with $\mathrm{CH}_{3} \mathrm{CN}$, concentrated at room temperature and immediately subjected to acid-catalyzed condensation with 6 (Western half) followed by metal-mediated oxidative cyclization. The resulting $\mathbf{Z n C}-\mathbf{T}^{5}$ was obtained in $20 \%$ yield.

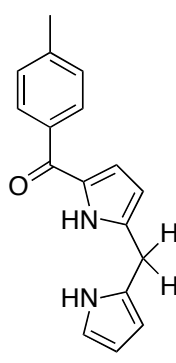

S9
(1) NBS, THF $-78^{\circ} \mathrm{C}$

(2) $\mathrm{NaBH}_{4}, \mathrm{THF} / \mathrm{MeOH}(3: 1), \mathrm{RT}$

(3) $\mathrm{NH}_{4} \mathrm{Cl}$ aq.

(4) 6, TFA, $\mathrm{CH}_{3} \mathrm{CN}, \mathrm{RT}$

(5) 2,2,6,6-tetramethylpiperidine $\mathrm{Zn}(\mathrm{OAc})_{2}, \mathrm{AgOTF}, \mathrm{CH}_{3} \mathrm{CN}$, reflux $20 \%$

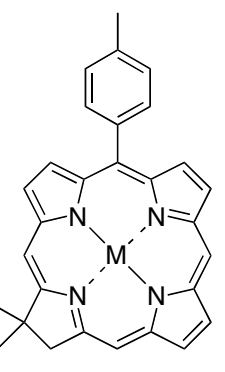

$\mathrm{ZnC}-\mathrm{T}^{5}(\mathrm{M}=\mathrm{Zn})$
(1) TFA, $\mathrm{CH}_{2} \mathrm{Cl}_{2}$

$\underset{84 \%}{\stackrel{(2) \mathrm{TEA}}{ }}$ FbC-T ${ }^{5}(\mathrm{M}=\mathrm{H}, \mathrm{H})$ 


\section{III. ${ }^{1}$ H NMR Spectra of Selected Bromochlorins.}
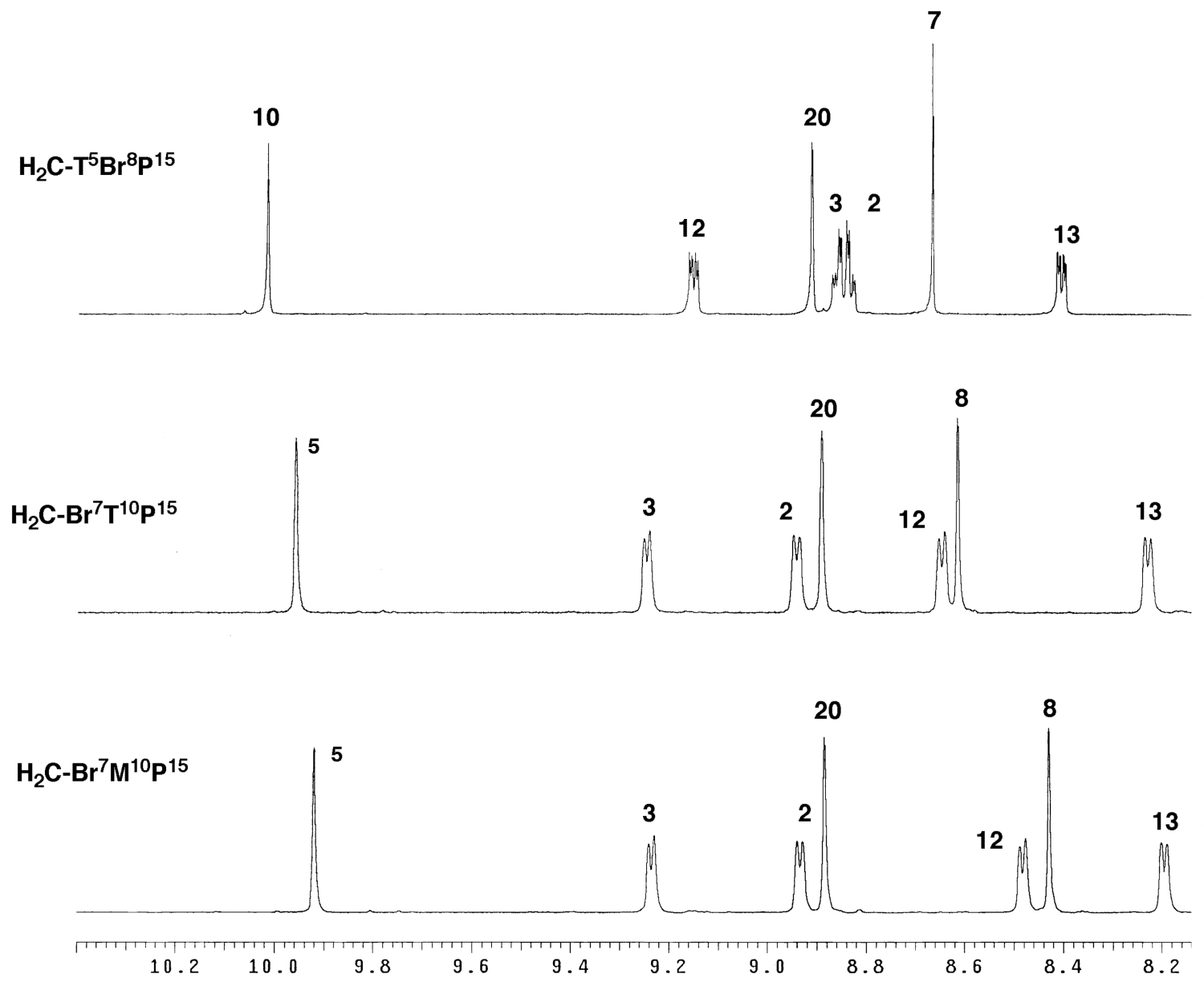

\section{Experimental Section}

General. ${ }^{1} \mathrm{H}$ NMR (400 MHz) and ${ }^{13} \mathrm{C}$ NMR (100 MHz) spectra were collected at room temperature in $\mathrm{CDCl}_{3}$ unless noted otherwise. Absorption spectra were obtained in toluene at room temperature. Chlorins were analyzed by laser desorption mass spectrometry (LD-MS) in the absence of a matrix. ${ }^{\mathrm{S} 8}$ Fast atom bombardment mass spectrometry (FAB-MS) data are reported for the molecule ion or protonated molecule ion. Melting points are uncorrected. All commercially available materials were used as received. All palladium-coupling reactions were carried out using standard Schlenk-line techniques. Toluene and triethylamine (TEA) were degassed with argon for 5 min prior to use. THF was distilled from $\mathrm{Na}$ /benzophenone as required. All bromination reactions (except noted otherwise) were performed using a freshly prepared $0.10 \mathrm{M}$ solution of NBS in THF.

Noncommercial Compounds. Compounds $1,{ }^{58} 4,{ }^{56} \mathbf{6},{ }^{17} \mathbf{8 a},{ }^{61} \mathbf{8 b},{ }^{14} \mathbf{1 0},{ }^{15} \mathbf{F b C}-\mathbf{P}^{15},{ }^{11}$ FbC-M ${ }^{10} \mathbf{B r}^{15},{ }^{11} \mathbf{S 1},{ }^{4} \mathbf{S 4},{ }^{\mathrm{S} 6} \mathbf{S 6},{ }^{\mathrm{S} 7}$ and $\mathbf{S 9}{ }^{10}$ were prepared following literature procedures.

4-Bromo-2-formyl-5-(1,3-dithiolan-2-yl)pyrrole (S2). A solution of S1 (400 mg, 2.00 $\mathrm{mmol})$ in dry THF $(20 \mathrm{~mL})$ at $-78^{\circ} \mathrm{C}$ under argon was treated with NBS (356 mg, $\left.2.00 \mathrm{mmol}\right)$. The reaction mixture was stirred for $1 \mathrm{~h}$ at $-78^{\circ} \mathrm{C}$. Hexanes was added to the reaction mixture at 
$-20{ }^{\circ} \mathrm{C}$. The reaction mixture was then allowed to warm to $0{ }^{\circ} \mathrm{C}$. Ethyl acetate was added. The organic layer was washed with water, dried $\left(\mathrm{Na}_{2} \mathrm{SO}_{4}\right)$ and concentrated. The resulting residue was chromatographed [silica, hexanes/ $\mathrm{CH}_{2} \mathrm{Cl}_{2} /$ ethyl acetate $(7: 2: 1)$ ] to afford a white solid (348 mg, 51\%): mp 92-94 ${ }^{\circ} \mathrm{C}(\mathrm{dec}) ;{ }^{1} \mathrm{H}$ NMR $\delta 3.31-3.46(\mathrm{~m}, 4 \mathrm{H}), 5.72(\mathrm{~s}, 1 \mathrm{H}), 6.90-6.91(\mathrm{~m}, 1 \mathrm{H})$, 9.42 (s, 1H), 9.63 (brs, 1H); ${ }^{13} \mathrm{C}$ NMR $\delta 39.8,48.0,110.5,122.1,132.6,140.6,179.1$; Anal. Calcd for $\mathrm{C}_{8} \mathrm{H}_{8} \mathrm{BrNOS}_{2}$ : C, 34.54; H, 2.90; N, 5.03. Found: C, 34.62; H, 2.94; N, 5.08.

2-Bromo-1-(1,3-dithiolan-2-yl)dipyrromethane (S3). A solution of S2 (323 mg, 1.16 mmol) in dry THF $(6.5 \mathrm{~mL})$ at $0{ }^{\circ} \mathrm{C}$ was treated with $\mathrm{LiBH}_{4}(101 \mathrm{mg}, 4.64 \mathrm{mmol})$ and the mixture was stirred for $1 \mathrm{~h}$. Water was added, and the resulting mixture was extracted with ethyl acetate. The organic layer was separated, dried $\left(\mathrm{Na}_{2} \mathrm{SO}_{4}\right)$, and concentrated to dryness. The resulting crude solid (4-bromo-2-hydroxymethylpyrrole) was dissolved in 1,4-dioxane (15 $\mathrm{mL})$, whereupon pyrrole $(1.30 \mathrm{~mL}, 17.4 \mathrm{mmol})$ was added. The solution was treated with $10 \%$ aqueous $\mathrm{HCl}(1.5 \mathrm{~mL})$, and the mixture was stirred at room temperature. The reaction was monitored by TLC [silica, hexanes/ethyl acetate (3:1)]. After $30 \mathrm{~min}$, the reaction mixture was quenched by the addition of saturated aqueous $\mathrm{NaHCO}_{3}$. The resulting mixture was extracted with $\mathrm{CH}_{2} \mathrm{Cl}_{2}$. The organic extract was washed with water, dried $\left(\mathrm{Na}_{2} \mathrm{SO}_{4}\right)$, and concentrated. The resulting solid was purified by column chromatography [silica, hexanes $/ \mathrm{CH}_{2} \mathrm{Cl}_{2} /$ ethyl acetate $(7: 2: 1)$ ], affording a brown solid $(271 \mathrm{mg}, 71 \%)$ : $\mathrm{mp} 83-85{ }^{\circ} \mathrm{C}(\mathrm{dec}) ;{ }^{1} \mathrm{H}$ NMR $\delta 3.25-$ $3.44(\mathrm{~m}, 4 \mathrm{H}), 3.91(\mathrm{~s}, 2 \mathrm{H}), 5.84(\mathrm{~s}, 1 \mathrm{H}), 5.93-5.94(\mathrm{~m}, 1 \mathrm{H}), 6.02-6.03(\mathrm{~m}, 1 \mathrm{H}), 6.14-6.17(\mathrm{~m}$, 1H), 6.68-6.70 (m, 1H), 7.98 (brs, 1H), 8.35 (brs, 1H); ${ }^{13} \mathrm{C}$ NMR $\delta 26.7,37.5,40.1,47.5,96.5$, 106.9, 108.7, 110.0, 117.5, 117.6, 131.3; Anal. Calcd for $\mathrm{C}_{12} \mathrm{H}_{13} \mathrm{BrN}_{2} \mathrm{~S}_{2}$ : C, 43.77; H, 3.98; N, 8.51. Found: C, 44.07; H, 3.98; N, 8.39.

2-Bromodipyrromethane (S5). A solution of $\mathbf{S 4}(382 \mathrm{mg}, 1.37 \mathrm{mmol})$ in dry THF (7.7 $\mathrm{mL})$ at $0{ }^{\circ} \mathrm{C}$ was treated with $\mathrm{LiBH}_{4}(119 \mathrm{mg}, 5.49 \mathrm{mmol})$. The mixture was stirred for $1 \mathrm{~h}$. Water was added, and the resulting mixture was extracted with ethyl acetate. The organic layer was separated, dried $\left(\mathrm{Na}_{2} \mathrm{SO}_{4}\right)$, and concentrated to dryness. The resulting crude solid was used in the next reaction. The crude product was dissolved in 1,4-dioxane $(17.7 \mathrm{~mL})$, whereupon pyrrole $(1.50 \mathrm{~mL}, 20.5 \mathrm{mmol})$ was added. The solution was treated with $10 \%$ aqueous $\mathrm{HCl}(1.7$ $\mathrm{mL}$ ), and the mixture was stirred for $30 \mathrm{~min}$ at room temperature. The reaction was monitored by TLC [silica, hexanes/ethyl acetate (3:1)]. After $30 \mathrm{~min}$, the reaction mixture was quenched by the addition of saturated aqueous $\mathrm{NaHCO}_{3}$. The resulting mixture was extracted with ethyl acetate. The organic extract was washed with $\mathrm{H}_{2} \mathrm{O}$, dried $\left(\mathrm{K}_{2} \mathrm{CO}_{3}\right)$, and concentrated. The resulting residue was purified by column chromatography [silica, hexanes $/ \mathrm{CH}_{2} \mathrm{Cl}_{2} /$ ethyl acetate (8:1:1)], affording a brown oil (402 mg, 58\%): ${ }^{1} \mathrm{H}$ NMR (THF- $\left.d_{8}\right) \delta 3.83(\mathrm{~s}, 2 \mathrm{H}), 5.81-5.84(\mathrm{~m}$, 2H), 5.91-5.93 (m, 1H), 6.55-6.58 (m, 2H), 9.68-9.90 (brs, 2H); ${ }^{13} \mathrm{C}$ NMR (THF- $\left.d_{8}\right) \delta 27.3$, 95.8, 106.8, 108.3, 109.3, 117.6, 117.7, 129.5, 132.1.

2-Methoxycarbonyl-9-decylthio-5-p-tolyldipyrromethane (S8). Following a reported procedure, ${ }^{4} p$-tolylmagnesium bromide $(1.0 \mathrm{M}, 0.760 \mathrm{~mL})$ was added to a solution of $\mathbf{S 6}(152$ $\mathrm{mg}, 0.690 \mathrm{mmol}$ ) in $5.0 \mathrm{~mL}$ of $\mathrm{THF}$ at $0{ }^{\circ} \mathrm{C}$. The reaction mixture was allowed to warm to room temperature with stirring for $3 \mathrm{~h}$. The reaction mixture was diluted with ethyl acetate, and then quenched by the addition of saturated aqueous $\mathrm{NH}_{4} \mathrm{Cl}$. The resulting mixture was washed with water. The organic layer was separated, dried $\left(\mathrm{Na}_{2} \mathrm{SO}_{4}\right)$ and concentrated. The resulting residue was purified by column chromatography [silica, hexanes $/ \mathrm{CH}_{2} \mathrm{Cl}_{2} /$ ethyl acetate $(7: 2: 1 \rightarrow 4: 3: 3 \rightarrow 1: 2: 2)$ ], affording the pyrrole-carbinol $\mathbf{S} 7$ as a thick oil (137 $\mathrm{mg}, 81 \%)$. Samples of $\mathbf{S} 7$ (137 mg, $0.558 \mathrm{mmol}$ ) and 2-(decylthio)pyrrole (134 $\mathrm{mg}, 0.558 \mathrm{mmol}$ ) were dissolved in toluene $(5.5 \mathrm{~mL})$. The solution was treated with $\mathrm{InCl}_{3}(12.3 \mathrm{mg}, 0.0558 \mathrm{mmol})$ at room 
temperature for $30 \mathrm{~min}$. The reaction mixture was diluted with ethyl acetate. The resulting mixture was washed with saturated $\mathrm{NaHCO}_{3}$ and $\mathrm{H}_{2} \mathrm{O}$. The organic layer was separated, dried $\left(\mathrm{Na}_{2} \mathrm{SO}_{4}\right)$, and concentrated. The resulting residue was purified by column chromatography [silica, hexanes $/ \mathrm{CH}_{2} \mathrm{Cl}_{2} /$ ethyl acetate $(7: 2: 1)$ ], affording a yellow oil $(162 \mathrm{mg}, 62 \%):{ }^{1} \mathrm{H} \mathrm{NMR} \delta$ 0.86-0.89 (m, 3H), 1.24-1.32 (m, 14H), 1.45-1.52 (m, 2H), 2.33 (s, 3H), 2.57 (t, J = 7.2 Hz, 2H), $3.75(\mathrm{~s}, 3 \mathrm{H}), 5.33(\mathrm{~s}, 1 \mathrm{H}), 5.85-5.87(\mathrm{~m}, 1 \mathrm{H}), 6.24-6.26(\mathrm{~m}, 1 \mathrm{H}), 6.29-6.30(\mathrm{~m}, 1 \mathrm{H}), 7.05$ $(\mathrm{d}, J=8.4 \mathrm{~Hz}, 2 \mathrm{H}), 7.12(\mathrm{~d}, J=8.4 \mathrm{~Hz}, 2 \mathrm{H}), 7.27-7.29(\mathrm{~m}, 1 \mathrm{H}), 8.00$ (brs, $1 \mathrm{H}), 8.42$ (brs, $1 \mathrm{H})$; ${ }^{13} \mathrm{C}$ NMR $\delta 14.3,21.2,22.8,28.6,29.5,29.8,30.0,32.1,38.1,43.7,51.2,108.5,109.2,116.3$, $116.8,119.4,123.5,128.3,129.7,133.8,134.4$, 137.2, 137.8, 165.7; FAB-MS obsd 466.2663, calcd $466.2654\left(\mathrm{C}_{28} \mathrm{H}_{38} \mathrm{~N}_{2} \mathrm{O}_{2} \mathrm{~S}\right)$.

Streamlined Synthesis of Zn(II)-17,18-Dihydro-18,18-dimethyl-5-p-tolylporphyrin $\left(\mathbf{Z n C}-\mathbf{T}^{\mathbf{5}}\right)$. A solution of 1-(4-methylbenzoyl)dipyrromethane S9 (0.264 g, $\left.1.00 \mathrm{mmol}\right)$ in THF $(20 \mathrm{~mL})$ was treated at $-78{ }^{\circ} \mathrm{C}$ with NBS $(0.178 \mathrm{~g}, 1.00 \mathrm{mmol})$. The resulting mixture was stirred at $-78{ }^{\circ} \mathrm{C}$ for $1 \mathrm{~h}$, then $\mathrm{MeOH}(20 \mathrm{~mL})$ and THF $(40 \mathrm{~mL})$ were added, and the reaction mixture was allowed to warm to room temperature. A sample of $\mathrm{NaBH}_{4}(0.756 \mathrm{~g}, 20.0 \mathrm{mmol})$ was added portionwise. The resulting mixture was stirred for $30 \mathrm{~min}$, at which point TLC analysis [silica, $\mathrm{CH}_{2} \mathrm{Cl}_{2}$ /ethyl acetate $(5: 1)$ ] showed the presence of starting material. Another batch of solid $\mathrm{NaBH}_{4}(1.51 \mathrm{~g}, 40.0 \mathrm{mmol})$ was added portionwise. The resulting mixture was stirred for $30 \mathrm{~min}$ and quenched by the addition of saturated aqueous $\mathrm{NH}_{4} \mathrm{Cl}$. The resulting mixture was extracted with diethyl ether. The organic extract was washed (water and brine) and dried $\left(\mathrm{K}_{2} \mathrm{CO}_{3}\right)$. Anhydrous $\mathrm{CH}_{3} \mathrm{CN}(10 \mathrm{~mL})$ was added. The volatile components (mostly diethyl ether) were removed under reduced pressure without heating. (Note that complete evaporation of solvent resulted in extensive decomposition of the carbinol and drastically reduced the yield of $\mathbf{Z n C}-\mathbf{T}^{\mathbf{5}}$.) The resulting solution was treated with $\mathbf{6}$ (0.190 g, $\left.1.00 \mathrm{mmol}\right)$ and TFA $(77 \mu \mathrm{L}, 1.0 \mathrm{mmol})$. The resulting mixture was stirred at room temperature for $30 \mathrm{~min}$ and then treated with 2,2,6,6-tetramethylpiperidine $(5.06 \mathrm{~mL}, 30.0 \mathrm{mmol})$ and $\mathrm{CH}_{3} \mathrm{CN}(90 \mathrm{~mL})$. Samples of $\mathrm{Zn}(\mathrm{OAc})_{2}(2.76 \mathrm{~g}, 15.0 \mathrm{mmol})$ and $\mathrm{AgOTf}(0.770 \mathrm{~g}, 3.00 \mathrm{mmol})$ were added. The resulting mixture was refluxed exposed to air for $24 \mathrm{~h}$. Chromatography [silica, hexanes $/ \mathrm{CH}_{2} \mathrm{Cl}_{2}$ $(1: 1)$ ] afforded a blue-green solid $(99 \mathrm{mg}, 20 \%)$. The characterization data $\left({ }^{1} \mathrm{H} \mathrm{NMR}, \mathrm{LD}-\mathrm{MS}\right.$, FAB-MS and UV-vis) were consistent with those reported previously. ${ }^{10}$

17,18-Dihydro-18,18-dimethyl-5-p-tolylporphyrin (FbC- $\mathbf{T}^{\mathbf{5}}$ ). Following a reported procedure, ${ }^{11}$ a solution of $\mathbf{Z n C}-\mathbf{T}^{\mathbf{5}}(99 \mathrm{mg}, 0.20 \mathrm{mmol})$ in $\mathrm{CH}_{2} \mathrm{Cl}_{2}(100 \mathrm{~mL})$ was treated with TFA $(0.770 \mathrm{~mL}, 10.0 \mathrm{mmol})$. The resulting mixture was stirred for $1 \mathrm{~h}$. The mixture was neutralized with excess TEA $(5 \mathrm{~mL})$. The mixture was concentrated and chromatographed [silica, hexanes $/ \mathrm{CH}_{2} \mathrm{Cl}_{2}(1: 1)$ ] to afford a green solid (72 $\mathrm{mg}, 84 \%$ ). The characterization data ( ${ }^{1} \mathrm{H}$ NMR, LD-MS, FAB-MS and UV-vis) were consistent with those reported previously. ${ }^{11}$ 


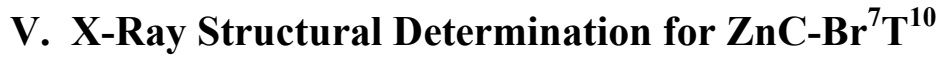

A single-crystal X-ray structure of $\mathbf{Z n C}-\mathbf{B r}^{7} \mathbf{T}^{\mathbf{1 0}}$ was determined (Figure S1).

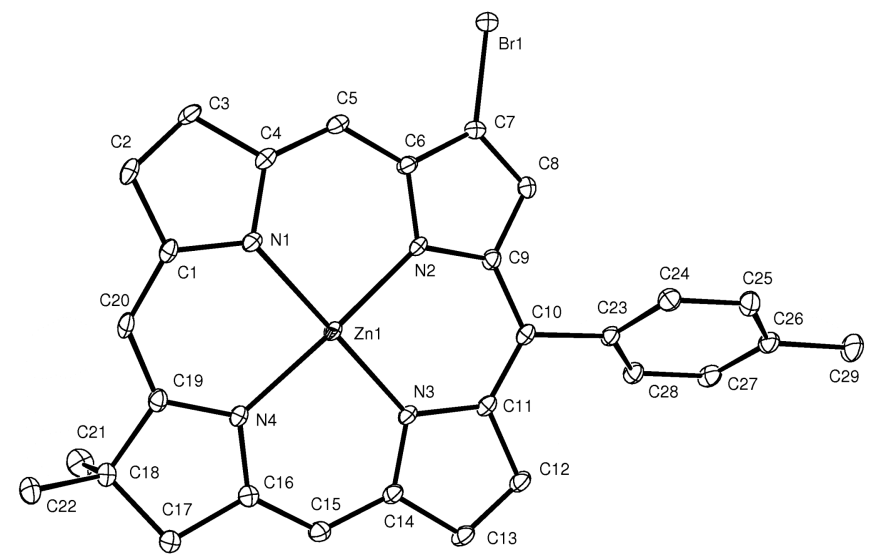

Figure S1. ORTEP drawing of $\mathbf{Z n C - B r} \mathbf{T}^{\mathbf{7}} \mathbf{1}^{\mathbf{1 0}}$ showing naming and numbering scheme. Ellipsoids are at the $50 \%$ probability level and hydrogen atoms are omitted for clarity.

Sample. A sample of $\mathbf{Z n C}-\mathbf{B r}^{7} \mathbf{T}^{\mathbf{1 0}}$ in $\mathrm{CDCl}_{3}$ at room temperature was crystallized by slow evaporation.

Data Collection. The sample was mounted on 20-micron nylon loop with a small amount of immersion oil. All X-ray measurements were made on a Bruker-Nonius X8 Apex2 CCD diffractometer at $110 \mathrm{~K}$. The unit cell dimensions were determined from a symmetry constrained fit of 9918 reflections with $4.74^{\circ}<2$ (theta) $<66.88^{\circ}$. The data collection strategy was a number of $\omega$ and $\varphi$ scans which collected data up to $70.0^{\circ}$ (2(theta)). The frame integration was performed using SAINT $+{ }^{\mathrm{S} 9}$ The resulting raw data was scaled and absorption corrected using a multi-scan averaging of symmetry equivalent data using SADABS. ${ }^{\text {S10 }}$

Structure Solution and Refinement. The structure was solved by direct methods using the SIR92 program. ${ }^{\text {S11 }}$ All non-hydrogen atoms were obtained from the initial E-map. The hydrogen atoms were introduced at idealized positions and were allowed to ride on the parent carbon atoms. The structural model was fit to the data using full matrix least-squares based on F. The calculated structure factors included corrections for anomalous dispersion from the usual tabulation. The structure was refined using the LSTSQ program from NRCVAX, ${ }^{\mathrm{S} 12}$ graphic plots were produced using the ORTEP implementation included in the NRCVAX crystallographic program suite. Additional information and other relevant literature references can be found in the reference section of the Facility's Web page (http://www.xray.ncsu.edu). 


\begin{tabular}{|c|c|}
\hline \multicolumn{2}{|c|}{ Crystallographic data and structure refinement for $\mathbf{Z n C}-\mathrm{Br}^{7} \mathbf{T}^{10}$} \\
\hline Formula & $\mathrm{C}_{30} \mathrm{H}_{24} \mathrm{BrCl}_{3} \mathrm{~N}_{4} \mathrm{Zn}$ \\
\hline Formula Weight $(\mathrm{g} / \mathrm{mol})$ & 692.18 \\
\hline Crystal Dimensions $(\mathrm{mm})$ & $0.42 \times 0.16 \times 0.02$ \\
\hline Crystal Color and Habit & dark red plate \\
\hline Crystal System & triclinic \\
\hline Space Group & $\mathrm{P}-1$ \\
\hline Temperature, $\mathrm{K}$ & 110 \\
\hline$a, \AA$ & $9.7091(4)$ \\
\hline$b, \AA$ & $9.8972(3)$ \\
\hline$c, \AA$ & $15.0704(5)$ \\
\hline$\alpha,^{\circ}$ & $76.1630(19)$ \\
\hline$\beta$, & $77.145(2)$ \\
\hline$\gamma,{ }^{\circ}$ & $78.0220(18)$ \\
\hline $\mathrm{V}, \AA^{3}$ & $1352.55(8)$ \\
\hline Number of reflections to determine final unit cell & 9918 \\
\hline Min and Max $2 \theta$ for cell determination, ${ }^{\circ}$ & $4.74,66.88$ \\
\hline $\mathrm{Z}$ & 2 \\
\hline $\mathrm{F}(000)$ & 697.37 \\
\hline$\rho(\mathrm{g} / \mathrm{cm})$ & 1.700 \\
\hline$\lambda, \AA,(\mathrm{MoK} \alpha)$ & 0.71073 \\
\hline$\mu,\left(\mathrm{cm}^{-1}\right)$ & 2.72 \\
\hline Diffractometer Type & Bruker-Nonius X8 Apex2 \\
\hline Scan Type(s) & omega and phi scans \\
\hline Max $2 \theta$ for data collection, ${ }^{\circ}$ & 70.0 \\
\hline Measured fraction of data & 0.96 \\
\hline Number of reflections measured & 67870 \\
\hline Unique reflections measured & 11398 \\
\hline $\mathrm{R}_{\text {merge }}$ & 0.037 \\
\hline Number of reflections included in refinement & 8914 \\
\hline Cut off Threshold Expression & Inet $>1.0$ sigma(Inet) \\
\hline Structure refined using & full matrix least-squares using $\mathrm{F}$ \\
\hline Weighting Scheme & $1 /\left(\operatorname{sigma}^{2}(\mathrm{~F})+0.0004 \mathrm{~F}^{2}\right)$ \\
\hline Number of parameters in least-squares & 352 \\
\hline $\mathrm{R}_{\mathrm{f}}$ & 0.043 \\
\hline $\mathrm{R}_{\mathrm{w}}$ & 0.042 \\
\hline $\mathrm{R}_{\mathrm{f}}$ (all data) & 0.072 \\
\hline $\mathrm{R}_{\mathrm{w}}$ (all data) & 0.043 \\
\hline
\end{tabular}




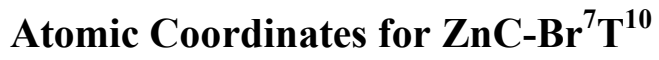

\begin{tabular}{|c|c|c|c|c|}
\hline Atom & $\mathrm{X}$ & $\mathrm{Y}$ & Z & $\mathrm{U}_{\text {iso/equiv }}$ \\
\hline $\mathrm{Zn} 1$ & $0.860912(20)$ & $0.02009(2)$ & $0.941256(14)$ & $0.01224(9)$ \\
\hline N1 & $1.01771(15)$ & $-0.15355(16)$ & $0.94975(10)$ & $0.0127(6)$ \\
\hline $\mathrm{N} 2$ & $0.74290(15)$ & $-0.07097(15)$ & $1.06374(10)$ & $0.0117(6)$ \\
\hline N3 & $0.69933(15)$ & $0.18405(15)$ & $0.92843(10)$ & $0.0120(6)$ \\
\hline N4 & $0.96731(15)$ & $0.09756(16)$ & $0.80672(10)$ & $0.0127(6)$ \\
\hline $\mathrm{C} 1$ & $1.13127(17)$ & $-0.18446(19)$ & $0.88108(12)$ & $0.0123(8)$ \\
\hline $\mathrm{C} 2$ & $1.20438(18)$ & $-0.32489(20)$ & $0.90809(13)$ & $0.0152(8)$ \\
\hline $\mathrm{C} 3$ & $1.13234(18)$ & $-0.38202(19)$ & $0.99204(12)$ & $0.0145(8)$ \\
\hline $\mathrm{C} 4$ & $1.01323(18)$ & $-0.27614(19)$ & $1.01760(12)$ & $0.0129(8)$ \\
\hline $\mathrm{C} 5$ & $0.90374(18)$ & $-0.29887(19)$ & $1.09246(12)$ & $0.0131(8)$ \\
\hline $\mathrm{C} 6$ & $0.77678(17)$ & $-0.20542(18)$ & $1.11296(11)$ & $0.0118(8)$ \\
\hline $\mathrm{C} 7$ & $0.65899(18)$ & $-0.23522(19)$ & $1.18621(12)$ & $0.0132(8)$ \\
\hline $\mathrm{Br} 1$ & $0.650873(19)$ & $-0.407833(19)$ & $1.271016(12)$ & $0.01638(8)$ \\
\hline $\mathrm{C} 8$ & $0.55434(18)$ & $-0.11954(19)$ & $1.18267(12)$ & $0.0134(8)$ \\
\hline $\mathrm{C} 9$ & $0.60941(17)$ & $-0.01676(19)$ & $1.10630(11)$ & $0.0121(8)$ \\
\hline $\mathrm{C} 10$ & $0.53590(17)$ & $0.12129(18)$ & $1.07768(12)$ & $0.0116(7)$ \\
\hline $\mathrm{C} 11$ & $0.57871(17)$ & $0.21331(18)$ & $0.99522(12)$ & $0.0123(8)$ \\
\hline $\mathrm{C} 12$ & $0.50733(18)$ & $0.35434(19)$ & $0.96506(12)$ & $0.0145(8)$ \\
\hline $\mathrm{C} 13$ & $0.58235(18)$ & $0.40807(19)$ & $0.88152(12)$ & $0.0146(8)$ \\
\hline $\mathrm{C} 14$ & $0.70180(18)$ & $0.30085(18)$ & $0.85949(12)$ & $0.0126(8)$ \\
\hline $\mathrm{C} 15$ & $0.80447(19)$ & $0.31879(19)$ & $0.77758(12)$ & $0.0148(8)$ \\
\hline $\mathrm{C} 16$ & $0.92491(18)$ & $0.22483(19)$ & $0.75305(12)$ & $0.0138(8)$ \\
\hline C17 & $1.03218(19)$ & $0.2529(2)$ & $0.66438(12)$ & $0.0173(8)$ \\
\hline $\mathrm{C} 18$ & $1.11807(18)$ & $0.10693(19)$ & $0.65696(12)$ & $0.0146(8)$ \\
\hline C19 & $1.08663(17)$ & $0.02885(19)$ & $0.75814(12)$ & $0.0128(8)$ \\
\hline $\mathrm{C} 20$ & $1.16283(17)$ & $-0.09807(19)$ & $0.79315(12)$ & $0.0137(8)$ \\
\hline $\mathrm{C} 21$ & $1.0538(2)$ & $0.0359(2)$ & $0.59900(13)$ & $0.0217(10)$ \\
\hline $\mathrm{C} 22$ & $1.27684(19)$ & $0.1100(2)$ & $0.61655(13)$ & $0.0176(9)$ \\
\hline $\mathrm{C} 23$ & $0.40634(17)$ & $0.17243(18)$ & $1.14389(12)$ & $0.0122(7)$ \\
\hline $\mathrm{C} 24$ & $0.41664(18)$ & $0.17173(20)$ & $1.23458(12)$ & $0.0154(8)$ \\
\hline $\mathrm{C} 25$ & $0.29882(20)$ & $0.2246(2)$ & $1.29612(13)$ & $0.0182(8)$ \\
\hline C26 & $0.16704(19)$ & $0.27820(20)$ & $1.26883(13)$ & $0.0164(8)$ \\
\hline $\mathrm{C} 27$ & $0.15657(18)$ & $0.27732(20)$ & $1.17854(13)$ & $0.0159(8)$ \\
\hline $\mathrm{C} 28$ & $0.27415(18)$ & $0.22504(19)$ & $1.11718(12)$ & $0.0140(8)$ \\
\hline C29 & $0.0401(2)$ & $0.3375(2)$ & $1.33437(14)$ & $0.0238(9)$ \\
\hline $\mathrm{C} 1 \mathrm{~s}$ & $0.5952(2)$ & $0.2907(2)$ & $0.52449(13)$ & $0.0221(9)$ \\
\hline $\mathrm{Cl1s}$ & $0.71127(6)$ & $0.40979(7)$ & $0.51571(4)$ & $0.0353(3)$ \\
\hline $\mathrm{Cl} 2 \mathrm{~s}$ & $0.66030(6)$ & $0.12100(6)$ & $0.58216(4)$ & $0.0322(3)$ \\
\hline $\mathrm{Cl3s}$ & $0.56934(6)$ & $0.28571(6)$ & $0.41328(4)$ & $0.0291(3)$ \\
\hline $\mathrm{H} 2$ & 1.289 & -0.370 & 0.873 & 0.0249 \\
\hline H3 & 1.156 & -0.475 & 1.028 & 0.0239 \\
\hline H5 & 0.916 & -0.388 & 1.135 & 0.0228 \\
\hline H8 & 0.463 & -0.109 & 1.223 & 0.0229 \\
\hline $\mathrm{H} 12$ & 0.422 & 0.402 & 0.998 & 0.0240 \\
\hline H13 & 0.561 & 0.500 & 0.844 & 0.0242 \\
\hline
\end{tabular}




$\begin{array}{lllll}\mathrm{H} 15 & 0.788 & 0.406 & 0.734 & 0.0240 \\ \mathrm{H} 17 \mathrm{a} & 0.985 & 0.292 & 0.612 & 0.0257 \\ \mathrm{H} 17 \mathrm{~b} & 1.093 & 0.316 & 0.668 & 0.0257 \\ \mathrm{H} 20 & 1.248 & -0.132 & 0.753 & 0.0234 \\ \mathrm{H} 21 \mathrm{a} & 0.954 & 0.034 & 0.625 & 0.0322 \\ \mathrm{H} 21 \mathrm{~b} & 1.064 & 0.088 & 0.536 & 0.0322 \\ \mathrm{H} 21 \mathrm{c} & 1.103 & -0.059 & 0.600 & 0.0322 \\ \mathrm{H} 22 \mathrm{a} & 1.316 & 0.154 & 0.653 & 0.0269 \\ \mathrm{H} 22 \mathrm{~b} & 1.327 & 0.015 & 0.617 & 0.0269 \\ \mathrm{H} 22 \mathrm{c} & 1.287 & 0.163 & 0.554 & 0.0269 \\ \mathrm{H} 24 & 0.506 & 0.134 & 1.255 & 0.0248 \\ \mathrm{H} 25 & 0.309 & 0.223 & 1.358 & 0.0275 \\ \mathrm{H} 27 & 0.066 & 0.313 & 1.158 & 0.0253 \\ \mathrm{H} 28 & 0.264 & 0.226 & 1.055 & 0.0238 \\ \mathrm{H} 29 \mathrm{a} & 0.067 & 0.330 & 1.393 & 0.0317 \\ \mathrm{H} 29 \mathrm{~b} & 0.009 & 0.435 & 1.309 & 0.0317 \\ \mathrm{H} 29 \mathrm{c} & -0.037 & 0.285 & 1.343 & 0.0317 \\ \mathrm{H} 1 \mathrm{~s} & 0.505 & 0.323 & 0.560 & 0.0305\end{array}$

Bond Lengths for $\mathrm{ZnC}-\mathrm{Br}^{7} \mathrm{~T}^{10}$

$\begin{array}{llll}\text { Zn1-N1 } & 2.0452(14) & \mathrm{C} 15-\mathrm{C} 16 & 1.375(2) \\ \text { Zn1-N2 } & 2.0553(15) & \mathrm{C} 15-\mathrm{H} 15 & 0.96 \\ \text { Zn1-N3 } & 2.0149(14) & \mathrm{C} 16-\mathrm{C} 17 & 1.507(2) \\ \text { Zn1-N4 } & 2.1022(14) & \mathrm{C} 17-\mathrm{C} 18 & 1.526(3) \\ \mathrm{N} 1-\mathrm{C} 1 & 1.373(2) & \mathrm{C} 17-\mathrm{H} 17 \mathrm{a} & 0.96 \\ \mathrm{~N} 1-\mathrm{C} 4 & 1.389(2) & \mathrm{C} 17-\mathrm{H} 17 \mathrm{~b} & 0.96 \\ \mathrm{~N} 2-\mathrm{C} 6 & 1.373(2) & \mathrm{C} 18-\mathrm{C} 19 & 1.531(2) \\ \mathrm{N} 2-\mathrm{C} 9 & 1.373(2) & \mathrm{C} 18-\mathrm{C} 21 & 1.537(3) \\ \mathrm{N} 3-\mathrm{C} 11 & 1.394(2) & \mathrm{C} 18-\mathrm{C} 22 & 1.530(2) \\ \mathrm{N} 3-\mathrm{C} 14 & 1.357(2) & \mathrm{C} 19-\mathrm{C} 20 & 1.369(2) \\ \mathrm{N} 4-\mathrm{C} 16 & 1.362(2) & \mathrm{C} 20-\mathrm{H} 20 & 0.96 \\ \text { N4-C19 } & 1.369(2) & \mathrm{C} 21-\mathrm{H} 21 \mathrm{a} & 0.96 \\ \mathrm{C} 1-\mathrm{C} 2 & 1.432(2) & \mathrm{C} 21-\mathrm{H} 21 \mathrm{~b} & 0.96 \\ \mathrm{C} 1-\mathrm{C} 20 & 1.403(3) & \mathrm{C} 21-\mathrm{H} 21 \mathrm{c} & 0.96 \\ \mathrm{C} 2-\mathrm{C} 3 & 1.356(3) & \mathrm{C} 22-\mathrm{H} 22 \mathrm{a} & 0.96 \\ \mathrm{C} 2-\mathrm{H} 2 & 0.96 & \mathrm{C} 22-\mathrm{H} 22 \mathrm{~b} & 0.96 \\ \mathrm{C} 3-\mathrm{C} 4 & 1.441(2) & \mathrm{C} 22-\mathrm{H} 22 \mathrm{c} & 0.96 \\ \mathrm{C} 3-\mathrm{H} 3 & 0.96 & \mathrm{C} 23-\mathrm{C} 24 & 1.391(2) \\ \mathrm{C} 4-\mathrm{C} 5 & 1.378(2) & \mathrm{C} 23-\mathrm{C} 28 & 1.390(2) \\ \mathrm{C} 5-\mathrm{C} 6 & 1.402(2) & \mathrm{C} 24-\mathrm{C} 25 & 1.398(2) \\ \mathrm{C} 5-\mathrm{H} 5 & 0.96 & \mathrm{C} 24-\mathrm{H} 24 & 0.96 \\ \mathrm{C} 6-\mathrm{C} 7 & 1.427(2) & \mathrm{C} 25-\mathrm{C} 26 & 1.391(3) \\ \mathrm{C} 7-\mathrm{B} 1 & 1.8775(17) & \mathrm{C} 25-\mathrm{H} 25 & 0.96 \\ \mathrm{C} 7-\mathrm{C} 8 & 1.363(2) & \mathrm{C} 26-\mathrm{C} 27 & 1.389(3) \\ \mathrm{C} 8-\mathrm{C} 9 & 1.427(3) & \mathrm{C} 26-\mathrm{C} 29 & 1.508(2) \\ \mathrm{C} 8-\mathrm{H} 8 & \mathrm{C} 27-\mathrm{C} 28 & 1.393(2) \\ \mathrm{C} 9-\mathrm{C} 10 & 1.419(2) & \mathrm{C} 27-\mathrm{H} 27 & 0.96\end{array}$




$\begin{array}{llll}\mathrm{C} 10-\mathrm{C} 11 & 1.390(2) & \mathrm{C} 28-\mathrm{H} 28 & 0.96 \\ \mathrm{C} 10-\mathrm{C} 23 & 1.501(2) & \mathrm{C} 29-\mathrm{H} 29 \mathrm{a} & 0.96 \\ \mathrm{C} 11-\mathrm{C} 12 & 1.439(2) & \mathrm{C} 29-\mathrm{H} 29 \mathrm{~b} & 0.96 \\ \mathrm{C} 12-\mathrm{C} 13 & 1.353(3) & \mathrm{C} 29-\mathrm{H} 29 \mathrm{c} & 0.96 \\ \mathrm{C} 12-\mathrm{H} 12 & 0.96 & \mathrm{H} 1 \mathrm{~s}-\mathrm{C} 1 \mathrm{~s} & 0.96 \\ \mathrm{C} 13-\mathrm{C} 14 & 1.438(2) & \mathrm{C} 1 \mathrm{~s}-\mathrm{C} 11 \mathrm{~s} & 1.757(2) \\ \mathrm{C} 13-\mathrm{H} 13 & 0.96 & \mathrm{C} 1 \mathrm{~s}-\mathrm{C} 12 \mathrm{~s} & 1.755(2) \\ \mathrm{C} 14-\mathrm{C} 15 & 1.404(2) & \mathrm{C} 1 \mathrm{~s}-\mathrm{Cl3s} & 1.7624(20)\end{array}$

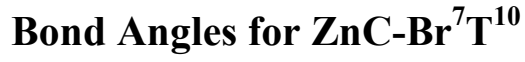

\begin{tabular}{|c|c|c|c|}
\hline N1-Zn1-N2 & $90.39(6)$ & N4-C16-C15 & $125.09(16)$ \\
\hline N1-Zn1-N3 & $175.92(6)$ & N4-C16-C17 & $110.80(14)$ \\
\hline $\mathrm{N} 1-\mathrm{Zn} 1-\mathrm{N} 4$ & $89.08(6)$ & C15-C16-C17 & $124.05(16)$ \\
\hline $\mathrm{N} 2-\mathrm{Zn} 1-\mathrm{N} 3$ & $89.32(6)$ & C16-C17-C18 & $103.41(15)$ \\
\hline $\mathrm{N} 2-\mathrm{Zn} 1-\mathrm{N} 4$ & $171.78(6)$ & C16-C17-H17a & 110.88 \\
\hline N3-Zn1-N4 & $90.61(6)$ & C16-C17-H17b & 110.94 \\
\hline Zn1-N1-C1 & $126.73(12)$ & C18-C17-H17a & 111.11 \\
\hline Zn1-N1-C4 & $126.01(11)$ & C18-C17-H17b & 110.92 \\
\hline C1-N1-C4 & $105.94(14)$ & H17a-C17-H17b & 109.47 \\
\hline Zn1-N2-C6 & $125.68(11)$ & C17-C18-C19 & $100.69(13)$ \\
\hline Zn1-N2-C9 & $127.12(12)$ & C17-C18-C21 & $110.75(15)$ \\
\hline C6-N2-C9 & $106.84(14)$ & C17-C18-C22 & $112.95(15)$ \\
\hline Zn1-N3-C11 & $127.26(11)$ & C19-C18-C21 & $107.33(15)$ \\
\hline Zn1-N3-C14 & $125.18(11)$ & C19-C18-C22 & $114.85(14)$ \\
\hline C11-N3-C14 & $106.46(14)$ & $\mathrm{C} 21-\mathrm{C} 18-\mathrm{C} 22$ & $109.86(14)$ \\
\hline Zn1-N4-C16 & $124.59(11)$ & N4-C19-C18 & $111.18(14)$ \\
\hline Zn1-N4-C19 & $126.28(12)$ & N4-C19-C20 & $124.75(16)$ \\
\hline C16-N4-C19 & $109.12(14)$ & C18-C19-C20 & $123.93(15)$ \\
\hline N1-C1-C2 & $110.06(15)$ & C1-C20-C19 & $127.58(15)$ \\
\hline $\mathrm{N} 1-\mathrm{C} 1-\mathrm{C} 20$ & $125.34(15)$ & C1-C20-H20 & 116.12 \\
\hline $\mathrm{C} 2-\mathrm{C} 1-\mathrm{C} 20$ & $124.26(15)$ & C19-C20-H20 & 116.30 \\
\hline $\mathrm{C} 1-\mathrm{C} 2-\mathrm{C} 3$ & $107.50(15)$ & C18-C21-H21a & 109.44 \\
\hline $\mathrm{C} 1-\mathrm{C} 2-\mathrm{H} 2$ & 126.26 & C18-C21-H21b & 109.30 \\
\hline $\mathrm{C} 3-\mathrm{C} 2-\mathrm{H} 2$ & 126.24 & C18-C21-H21c & 109.67 \\
\hline $\mathrm{C} 2-\mathrm{C} 3-\mathrm{C} 4$ & $106.87(15)$ & $\mathrm{H} 21 \mathrm{a}-\mathrm{C} 21-\mathrm{H} 21 \mathrm{~b}$ & 109.47 \\
\hline $\mathrm{C} 2-\mathrm{C} 3-\mathrm{H} 3$ & 126.78 & $\mathrm{H} 21 \mathrm{a}-\mathrm{C} 21-\mathrm{H} 21 \mathrm{c}$ & 109.5 \\
\hline $\mathrm{C} 4-\mathrm{C} 3-\mathrm{H} 3$ & 126.36 & $\mathrm{H} 21 \mathrm{~b}-\mathrm{C} 21-\mathrm{H} 21 \mathrm{c}$ & 109.47 \\
\hline $\mathrm{N} 1-\mathrm{C} 4-\mathrm{C} 3$ & $109.50(15)$ & C18-C22-H22a & 109.44 \\
\hline $\mathrm{N} 1-\mathrm{C} 4-\mathrm{C} 5$ & $125.38(15)$ & C18-C22-H22b & 109.45 \\
\hline $\mathrm{C} 3-\mathrm{C} 4-\mathrm{C} 5$ & $124.76(16)$ & C18-C22-H22c & 109.52 \\
\hline C4-C5-C6 & $126.73(16)$ & $\mathrm{H} 22 \mathrm{a}-\mathrm{C} 22-\mathrm{H} 22 \mathrm{~b}$ & 109.47 \\
\hline C4-C5-H5 & 116.58 & $\mathrm{H} 22 \mathrm{a}-\mathrm{C} 22-\mathrm{H} 22 \mathrm{c}$ & 109.47 \\
\hline C6-C5-H5 & 116.69 & $\mathrm{H} 22 \mathrm{~b}-\mathrm{C} 22-\mathrm{H} 22 \mathrm{c}$ & 109.47 \\
\hline N2-C6-C5 & $125.55(15)$ & $\mathrm{C} 10-\mathrm{C} 23-\mathrm{C} 24$ & $120.21(15)$ \\
\hline N2-C6-C7 & $108.43(14)$ & $\mathrm{C} 10-\mathrm{C} 23-\mathrm{C} 28$ & $122.10(15)$ \\
\hline $\mathrm{C} 5-\mathrm{C} 6-\mathrm{C} 7$ & $125.98(16)$ & C24-C23-C28 & $117.67(15)$ \\
\hline C6-C7-Br1 & $124.81(13)$ & C23-C24-C25 & $121.11(16)$ \\
\hline
\end{tabular}




\begin{tabular}{|c|c|c|c|}
\hline $\mathrm{C} 6-\mathrm{C} 7-\mathrm{C} 8$ & $108.78(15)$ & $\mathrm{C} 23-\mathrm{C} 24-\mathrm{H} 24$ & 119.35 \\
\hline $\mathrm{Br} 1-\mathrm{C} 7-\mathrm{C} 8$ & $126.40(13)$ & $\mathrm{C} 25-\mathrm{C} 24-\mathrm{H} 24$ & 119.54 \\
\hline $\mathrm{C} 7-\mathrm{C} 8-\mathrm{C} 9$ & $105.53(15)$ & $\mathrm{C} 24-\mathrm{C} 25-\mathrm{C} 26$ & $121.01(16)$ \\
\hline $\mathrm{C} 7-\mathrm{C} 8-\mathrm{H} 8$ & 127.28 & $\mathrm{C} 24-\mathrm{C} 25-\mathrm{H} 25$ & 119.60 \\
\hline $\mathrm{C} 9-\mathrm{C} 8-\mathrm{H} 8$ & 127.19 & $\mathrm{C} 26-\mathrm{C} 25-\mathrm{H} 25$ & 119.38 \\
\hline N2-C9-C8 & $110.39(15)$ & $\mathrm{C} 25-\mathrm{C} 26-\mathrm{C} 27$ & $117.78(15)$ \\
\hline N2-C9-C10 & $124.79(16)$ & $\mathrm{C} 25-\mathrm{C} 26-\mathrm{C} 29$ & $121.30(17)$ \\
\hline C8-C9-C10 & $124.81(15)$ & C27-C26-C29 & $120.91(16)$ \\
\hline C9-C10-C11 & $124.75(15)$ & C26-C27-C28 & $121.16(16)$ \\
\hline C9-C10-C23 & $116.90(15)$ & C26-C27-H27 & 119.23 \\
\hline $\mathrm{C} 11-\mathrm{C} 10-\mathrm{C} 23$ & $118.30(15)$ & $\mathrm{C} 28-\mathrm{C} 27-\mathrm{H} 27$ & 119.61 \\
\hline N3-C11-C10 & $125.32(15)$ & $\mathrm{C} 23-\mathrm{C} 28-\mathrm{C} 27$ & $121.26(16)$ \\
\hline N3-C11-C12 & $108.77(15)$ & $\mathrm{C} 23-\mathrm{C} 28-\mathrm{H} 28$ & 119.35 \\
\hline $\mathrm{C} 10-\mathrm{C} 11-\mathrm{C} 12$ & $125.91(15)$ & $\mathrm{C} 27-\mathrm{C} 28-\mathrm{H} 28$ & 119.39 \\
\hline C11-C12-C13 & $107.53(15)$ & C26-C29-H29a & 109.39 \\
\hline C11-C12-H12 & 126.17 & C26-C29-H29b & 109.56 \\
\hline C13-C12-H12 & 126.29 & C26-C29-H29c & 109.47 \\
\hline C12-C13-C14 & $106.87(15)$ & H29a-C29-H29b & 109.5 \\
\hline C12-C13-H13 & 126.78 & H29a-C29-H29c & 109.47 \\
\hline C14-C13-H13 & 126.35 & $\mathrm{H} 29 \mathrm{~b}-\mathrm{C} 29-\mathrm{H} 29 \mathrm{c}$ & 109.47 \\
\hline N3-C14-C13 & $110.36(15)$ & $\mathrm{H} 1 \mathrm{~s}-\mathrm{C} 1 \mathrm{~s}-\mathrm{C} 11 \mathrm{~s}$ & 108.10 \\
\hline N3-C14-C15 & $126.36(15)$ & $\mathrm{H} 1 \mathrm{~s}-\mathrm{C} 1 \mathrm{~s}-\mathrm{Cl} 2 \mathrm{~s}$ & 108.27 \\
\hline C13-C14-C15 & $123.29(16)$ & $\mathrm{H} 1 \mathrm{~s}-\mathrm{C} 1 \mathrm{~s}-\mathrm{C} 13 \mathrm{~s}$ & 108.38 \\
\hline C14-C15-C16 & $127.08(17)$ & $\mathrm{Cl} 1 \mathrm{~s}-\mathrm{C} 1 \mathrm{~s}-\mathrm{Cl} 2 \mathrm{~s}$ & $111.36(11)$ \\
\hline C14-C15-H15 & 116.47 & $\mathrm{Cl} 1 \mathrm{~s}-\mathrm{C} 1 \mathrm{~s}-\mathrm{Cl} 3 \mathrm{~s}$ & $110.33(11)$ \\
\hline C16-C15-H15 & 116.45 & $\mathrm{Cl} 2 \mathrm{~s}-\mathrm{C} 1 \mathrm{~s}-\mathrm{Cl} 3 \mathrm{~s}$ & $110.30(11)$ \\
\hline
\end{tabular}

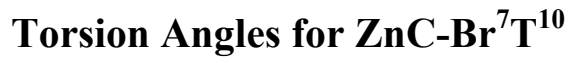

$\begin{array}{ll}\text { N2-Zn1-N1-C1 } & 168.4(3) \\ \text { N2-Zn1-N1-C4 } & 3.45(18) \\ \text { N3-Zn1-N1-C1 } & 82.4(2) \\ \text { N3-Zn1-N1-C4 } & -82.6(2) \\ \text { N4-Zn1-N1-C1 } & -3.35(18) \\ \text { N4-Zn1-N1-C4 } & -168.3(3) \\ \text { N1-Zn1-N2-C6 } & -5.15(18) \\ \text { N1-Zn1-N2-C9 } & -177.3(3) \\ \text { N3-Zn1-N2-C6 } & 170.8(3) \\ \text { N3-Zn1-N2-C9 } & -1.37(18) \\ \text { N4-Zn1-N2-C6 } & 81.2(2) \\ \text { N4-Zn1-N2-C9 } & -91.0(2) \\ \text { N1-Zn1-N3-C11 } & 96.5(2) \\ \text { N1-Zn1-N3-C14 } & -97.1(2) \\ \text { N2-Zn1-N3-C11 } & 10.46(18) \\ \text { N2-Zn1-N3-C14 } & 176.9(3) \\ \text { N4-Zn1-N3-C11 } & -177.8(3) \\ \text { N4-Zn1-N3-C14 } & -11.36(18) \\ \text { N1-Zn1-N4-C16 } & -176.9(3)\end{array}$

$\begin{array}{ll}\text { Br1-C7-C8-C9 } & -179.2(4) \\ \text { C7-C8-C9-N2 } & 1.3(2) \\ \text { C7-C8-C9-C10 } & -179.4(5) \\ \text { N2-C9-C10-C11 } & 10.0(2) \\ \text { N2-C9-C10-C23 } & -167.3(5) \\ \text { C8-C9-C10-C11 } & -169.2(5) \\ \text { C8-C9-C10-C23 } & 13.6(2) \\ \text { C9-C10-C11-N3 } & 0.17(19) \\ \text { C9-C10-C11-C12 } & -179.1(5) \\ \text { C23-C10-C11-N3 } & 177.3(4) \\ \text { C23-C10-C11-C12 } & -2.0(2) \\ \text { C9-C10-C23-C24 } & 55.1(3) \\ \text { C9-C10-C23-C28 } & -126.6(4) \\ \text { C11-C10-C23-C24 } & -122.3(4) \\ \text { C11-C10-C23-C28 } & 56.0(3) \\ \text { N3-C11-C12-C13 } & 0.7(2) \\ \text { C10-C11-C12-C13 } & -179.9(5) \\ \text { C11-C12-C13-C14 } & -0.4(2) \\ \text { C12-C13-C14-N3 } & 0.0(2)\end{array}$




\begin{tabular}{|c|c|c|c|}
\hline N1-Zn1-N4-C19 & $4.74(18)$ & C12-C13-C14-C15 & $-179.8(5)$ \\
\hline N2-Zn1-N4-C16 & $96.7(2)$ & N3-C14-C15-C16 & $-2.9(2)$ \\
\hline N2-Zn1-N4-C19 & $-81.7(2)$ & C13-C14-C15-C16 & $176.8(5)$ \\
\hline N3-Zn1-N4-C16 & $7.19(19)$ & C14-C15-C16-N4 & $-2.0(2)$ \\
\hline N3-Zn1-N4-C19 & $-171.2(3)$ & C14-C15-C16-C17 & $-178.7(5)$ \\
\hline Zn1-N1-C1-C2 & $-170.9(4)$ & N4-C16-C17-C18 & $18.2(2)$ \\
\hline Zn1-N1-C1-C20 & $2.72(16)$ & C15-C16-C17-C18 & $-164.6(5)$ \\
\hline C4-N1-C1-C2 & $-3.5(2)$ & C16-C17-C18-C19 & $-20.8(2)$ \\
\hline C4-N1-C1-C20 & $170.1(4)$ & C16-C17-C18-C21 & $92.6(4)$ \\
\hline Zn1-N1-C4-C3 & $171.0(4)$ & C16-C17-C18-C22 & $-143.7(4)$ \\
\hline Zn1-N1-C4-C5 & $-2.36(17)$ & C17-C18-C19-N4 & $18.9(2)$ \\
\hline C1-N1-C4-C3 & $3.4(2)$ & C17-C18-C19-C20 & $-165.2(5)$ \\
\hline C1-N1-C4-C5 & $-169.9(5)$ & C21-C18-C19-N4 & $-97.0(4)$ \\
\hline Zn1-N2-C6-C5 & $6.01(17)$ & C21-C18-C19-C20 & $78.9(3)$ \\
\hline Zn1-N2-C6-C7 & $-172.0(4)$ & C22-C18-C19-N4 & $140.5(4)$ \\
\hline C9-N2-C6-C5 & $179.5(4)$ & C22-C18-C19-C20 & $-43.6(3)$ \\
\hline C9-N2-C6-C7 & $1.5(2)$ & N4-C19-C20-C1 & $3.62(20)$ \\
\hline Zn1-N2-C9-C8 & $171.6(4)$ & C18-C19-C20-C1 & $-171.7(5)$ \\
\hline Zn1-N2-C9-C10 & $-7.70(16)$ & C10-C23-C24-C25 & $177.2(5)$ \\
\hline C6-N2-C9-C8 & $-1.8(2)$ & $\mathrm{C} 28-\mathrm{C} 23-\mathrm{C} 24-\mathrm{C} 25$ & $-1.2(2)$ \\
\hline C6-N2-C9-C10 & $179.0(4)$ & $\mathrm{C} 10-\mathrm{C} 23-\mathrm{C} 28-\mathrm{C} 27$ & $-177.4(5)$ \\
\hline Zn1-N3-C11-C10 & $-11.67(17)$ & $\mathrm{C} 24-\mathrm{C} 23-\mathrm{C} 28-\mathrm{C} 27$ & $1.0(2)$ \\
\hline Zn1-N3-C11-C12 & $167.8(4)$ & $\mathrm{C} 23-\mathrm{C} 24-\mathrm{C} 25-\mathrm{C} 26$ & $0.7(2)$ \\
\hline C14-N3-C11-C10 & $179.9(5)$ & $\mathrm{C} 24-\mathrm{C} 25-\mathrm{C} 26-\mathrm{C} 27$ & $0.1(2)$ \\
\hline C14-N3-C11-C12 & $-0.7(2)$ & C24-C25-C26-C29 & $-179.0(5)$ \\
\hline Zn1-N3-C14-C13 & $-168.3(4)$ & $\mathrm{C} 25-\mathrm{C} 26-\mathrm{C} 27-\mathrm{C} 28$ & $-0.3(2)$ \\
\hline Zn1-N3-C14-C15 & $11.47(17)$ & C29-C26-C27-C28 & $178.8(5)$ \\
\hline C11-N3-C14-C13 & $0.4(2)$ & $\mathrm{C} 26-\mathrm{C} 27-\mathrm{C} 28-\mathrm{C} 23$ & $-0.3(2)$ \\
\hline C11-N3-C14-C15 & $-179.8(4)$ & $\mathrm{Cl} 2 \mathrm{~s}-\mathrm{C} 1 \mathrm{~s}-\mathrm{Cl1} \mathrm{s}-\mathrm{Cl} 2 \mathrm{~s}$ & $0.00(7)$ \\
\hline Zn1-N4-C16-C15 & $-2.23(18)$ & $\mathrm{Cl} 2 \mathrm{~s}-\mathrm{C} 1 \mathrm{~s}-\mathrm{Cl1s}-\mathrm{Cl} 3 \mathrm{~s}$ & $122.8(2)$ \\
\hline Zn1-N4-C16-C17 & $174.9(4)$ & $\mathrm{Cl} 3 \mathrm{~s}-\mathrm{C} 1 \mathrm{~s}-\mathrm{Cl1s}-\mathrm{Cl} 2 \mathrm{~s}$ & $-122.8(2)$ \\
\hline C19-N4-C16-C15 & $176.4(5)$ & $\mathrm{Cl3s}-\mathrm{C} 1 \mathrm{~s}-\mathrm{Cl1s}-\mathrm{Cl} 3 \mathrm{~s}$ & $0.00(7)$ \\
\hline C19-N4-C16-C17 & $-6.5(2)$ & $\mathrm{Cl1s}-\mathrm{C} 1 \mathrm{~s}-\mathrm{Cl} 2 \mathrm{~s}-\mathrm{Cl1s}$ & $0.00(8)$ \\
\hline Zn1-N4-C19-C18 & $170.2(3)$ & $\mathrm{Cl1s}-\mathrm{C} 1 \mathrm{~s}-\mathrm{Cl} 2 \mathrm{~s}-\mathrm{Cl} 3 \mathrm{~s}$ & $-122.9(2)$ \\
\hline Zn1-N4-C19-C20 & $-5.63(17)$ & $\mathrm{Cl} 3 \mathrm{~s}-\mathrm{C} 1 \mathrm{~s}-\mathrm{Cl} 2 \mathrm{~s}-\mathrm{Cl1s}$ & $122.9(2)$ \\
\hline C16-N4-C19-C18 & $-8.4(2)$ & $\mathrm{Cl} 3 \mathrm{~s}-\mathrm{C} 1 \mathrm{~s}-\mathrm{Cl} 2 \mathrm{~s}-\mathrm{Cl} 3 \mathrm{~s}$ & $0.00(7)$ \\
\hline C16-N4-C19-C20 & $175.8(5)$ & $\mathrm{Cl1s}-\mathrm{C} 1 \mathrm{~s}-\mathrm{Cl} 3 \mathrm{~s}-\mathrm{Cl1s}$ & $0.00(8)$ \\
\hline N1-C1-C2-C3 & $2.2(2)$ & $\mathrm{Cl1s}-\mathrm{C} 1 \mathrm{~s}-\mathrm{Cl} 3 \mathrm{~s}-\mathrm{Cl} 2 \mathrm{~s}$ & $123.5(2)$ \\
\hline $\mathrm{C} 20-\mathrm{C} 1-\mathrm{C} 2-\mathrm{C} 3$ & $-171.5(5)$ & $\mathrm{Cl} 2 \mathrm{~s}-\mathrm{C} 1 \mathrm{~s}-\mathrm{Cl} 3 \mathrm{~s}-\mathrm{Cl1s}$ & $-123.5(2)$ \\
\hline N1-C1-C20-C19 & $-2.0(2)$ & $\mathrm{Cl} 2 \mathrm{~s}-\mathrm{C} 1 \mathrm{~s}-\mathrm{Cl} 3 \mathrm{~s}-\mathrm{Cl} 2 \mathrm{~s}$ & $0.00(7)$ \\
\hline C2-C1-C20-C19 & $170.7(5)$ & $\mathrm{C} 1 \mathrm{~s}-\mathrm{C} 11 \mathrm{~s}-\mathrm{Cl} 2 \mathrm{~s}-\mathrm{C} 1 \mathrm{~s}$ & $0.00(20)$ \\
\hline $\mathrm{C} 1-\mathrm{C} 2-\mathrm{C} 3-\mathrm{C} 4$ & $0.0(2)$ & $\mathrm{C} 1 \mathrm{~s}-\mathrm{Cl1s}-\mathrm{Cl} 2 \mathrm{~s}-\mathrm{Cl} 3 \mathrm{~s}$ & $33.78(15)$ \\
\hline $\mathrm{C} 2-\mathrm{C} 3-\mathrm{C} 4-\mathrm{N} 1$ & $-2.1(2)$ & $\mathrm{Cl} 3 \mathrm{~s}-\mathrm{Cl1s}-\mathrm{Cl} 2 \mathrm{~s}-\mathrm{C} 1 \mathrm{~s}$ & $-33.78(15)$ \\
\hline $\mathrm{C} 2-\mathrm{C} 3-\mathrm{C} 4-\mathrm{C} 5$ & $171.2(5)$ & $\mathrm{Cl} 3 \mathrm{~s}-\mathrm{Cl1} \mathrm{s}-\mathrm{Cl} 2 \mathrm{~s}-\mathrm{Cl} 3 \mathrm{~s}$ & $0.00(3)$ \\
\hline N1-C4-C5-C6 & $1.48(19)$ & $\mathrm{C} 1 \mathrm{~s}-\mathrm{Cl1s}-\mathrm{Cl3s}-\mathrm{C} 1 \mathrm{~s}$ & $0.00(20)$ \\
\hline $\mathrm{C} 3-\mathrm{C} 4-\mathrm{C} 5-\mathrm{C} 6$ & $-170.9(5)$ & $\mathrm{C} 1 \mathrm{~s}-\mathrm{Cl1s}-\mathrm{Cl} 3 \mathrm{~s}-\mathrm{Cl} 2 \mathrm{~s}$ & $-33.21(15)$ \\
\hline $\mathrm{C} 4-\mathrm{C} 5-\mathrm{C} 6-\mathrm{N} 2$ & $-3.5(2)$ & $\mathrm{Cl} 2 \mathrm{~s}-\mathrm{Cl1s}-\mathrm{Cl} 3 \mathrm{~s}-\mathrm{C} 1 \mathrm{~s}$ & $33.21(15)$ \\
\hline C4-C5-C6-C7 & $174.2(5)$ & $\mathrm{Cl} 2 \mathrm{~s}-\mathrm{Cl1} \mathrm{s}-\mathrm{Cl} 3 \mathrm{~s}-\mathrm{Cl} 2 \mathrm{~s}$ & $0.00(3)$ \\
\hline
\end{tabular}




$\begin{array}{llll}\text { N2-C6-C7-Br1 } & 178.1(4) & \mathrm{C} 1 \mathrm{~s}-\mathrm{Cl} 2 \mathrm{~s}-\mathrm{Cl} 3 \mathrm{~s}-\mathrm{C} 1 \mathrm{~s} & 0.00(20) \\ \text { N2-C6-C7-C8 } & -0.7(2) & \mathrm{C} 1 \mathrm{~s}-\mathrm{Cl} 2 \mathrm{~s}-\mathrm{Cl} 3 \mathrm{~s}-\mathrm{Cl1s} & 33.22(15) \\ \mathrm{C} 5-\mathrm{C} 6-\mathrm{C} 7-\mathrm{Br} 1 & 0.16(16) & \mathrm{Cl1s}-\mathrm{Cl} 2 \mathrm{~s}-\mathrm{Cl} 3 \mathrm{~s}-\mathrm{C} 1 \mathrm{~s} & -33.22(15) \\ \mathrm{C} 5-\mathrm{C} 6-\mathrm{C} 7-\mathrm{C} 8 & -178.7(5) & \mathrm{C} 11 \mathrm{~s}-\mathrm{Cl} 2 \mathrm{~s}-\mathrm{Cl} 3 \mathrm{~s}-\mathrm{Cl1s} & 0.00(4) \\ \text { C6-C7-C8-C9 } & -0.4(2) & & \end{array}$

\section{References}

(S1) (a) Vedejs, E.; Fuchs, P. L. J. Org. Chem. 1971, 36, 366-367. (b) Cadamuro, S.; Degani, I.; Fochi, R.; Gatti, A.; Piscopo, L. J. Chem. Soc. Perkin Trans. 1 1993, 2939-2943. (c) Cadamuro, S.; Degani, I.; Fochi, R.; Gatti, A.; Piscopo, L. J. Chem. Soc. Perkin Trans. 1 1996, 2365-2369. (d) Barnhurst, L. A.; Wan, Y.; Kutateladze, A. G. Org. Lett. 2000, 2, 799-801. (e) Vakalopoulos, A.; Hoffmann, H. M. R. Org. Lett. 2001, 3, 177-180. (f) Vakalopoulos, A.; Hoffmann, H. M. R. Org. Lett. 2001, 3, 2185-2188. (g) Fleming, F. F.; Funk, L.; Altundas, R.; Tu, Y. J. Org. Chem. 2001, 66, 6502-6504.

(S2) (a) Clezy, P. S.; Fookes, C. J. R. Aust. J. Chem. 1974, 27, 371-382. (b) Clezy, P. S.; Crowley, R. J.; Hai, T. T. Aust. J. Chem. 1982, 35, 411-421.

(S3) (a) Plantier-Royon, R.; Portella, C. Tetrahedron Lett. 1996, 37, 6113-6114. (b) Abell, A. D.; Nabbs, B. K.; Battersby, A. R. J. Org. Chem. 1998, 63, 8163-8169. (c) Biswas, G.; Ghorai, S.; Bhattacharjya, A. Org. Lett. 2006, 8, 313-316.

(S4) Wallace, D. M.; Leung, S. H.; Senge, M. O.; Smith, K. M. J. Org. Chem. 1993, 58, 72457257.

(S5) Liu, J.; Wong, C.-H. Tetrahedron Lett. 2002, 43, 4037-4039.

(S6) Anderson, H. J.; Lee, S.-F. Can. J. Chem. 1965, 43, 409-414.

(S7) Anderson, H. J.; Loader, C. E.; Foster, A. Can. J. Chem. 1980, 58, 2527-2530.

(S8) Srinivasan, N.; Haney, C. A.; Lindsey, J. S.; Zhang, W.; Chait, B. T. J. Porphyrins Phthalocyanines 1999, 3, 283-291.

(S9) Bruker-Nonius, SAINT+ version 7.34A, 2006, Bruker-Nonius, Madison, WI 53711, USA.

(S10) Bruker-Nonius, SADABS version 2.10, 2004, Bruker-Nonius, Madison, WI 53711, USA.

(S11) Altomare, A.; Burla, M. C.; Camalli, M.; Cascarano, G. L.; Giacovazzo, C.; Guagliardi, A.; Moliterni, A. G. G.; Polidori, G.; Spagna, R. J. Appl. Cryst. 1999, 32, 115-119.

(S12) Gabe, E. J.; Le Page, Y.; Charland, J.-P.; Lee, F. L.; White, P. S. J. Appl. Cryst. 1989, 22, 384-387.

\section{Spectral Data}

Assignment of the ${ }^{1} \mathbf{H}$ NMR Spectra. The structure of the 7-substituted chlorins was established in part by 2D NMR (H-H COSY and NOESY). The main features which allowed the full assignment of the resonances in ${ }^{1} \mathrm{H}$ NMR spectra include: (1) the geminal dimethyl groups at the pyrroline ring exhibit a NOE with $\mathrm{H}^{20}$; (2) the protons at the meso positions exhibit a NOE with the adjacent $\beta$-pyrrolic protons; and (3) the protons at the ortho positions of the meso-aryl substituents exhibit a NOE with the adjacent $\beta$-pyrrolic protons. 


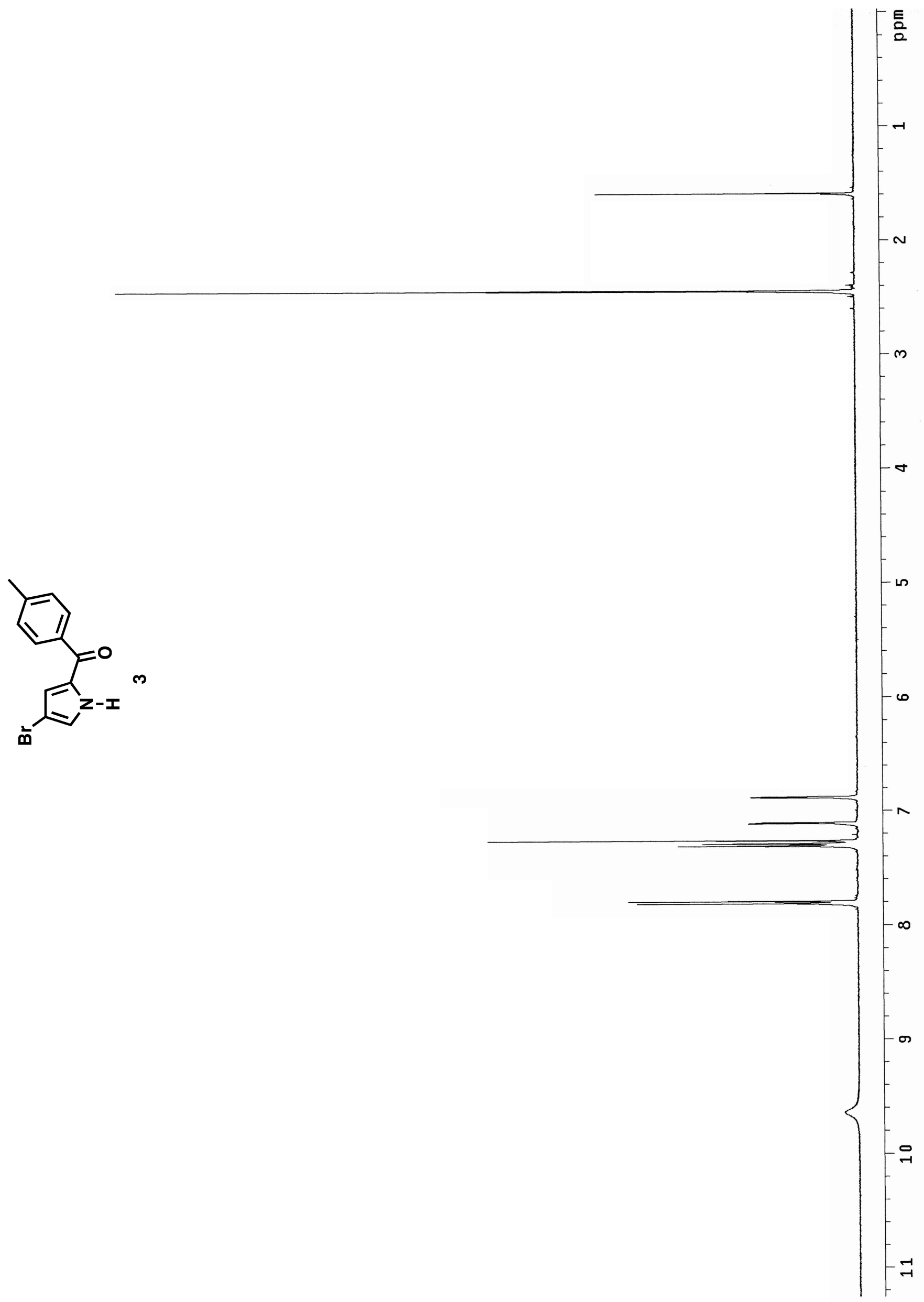




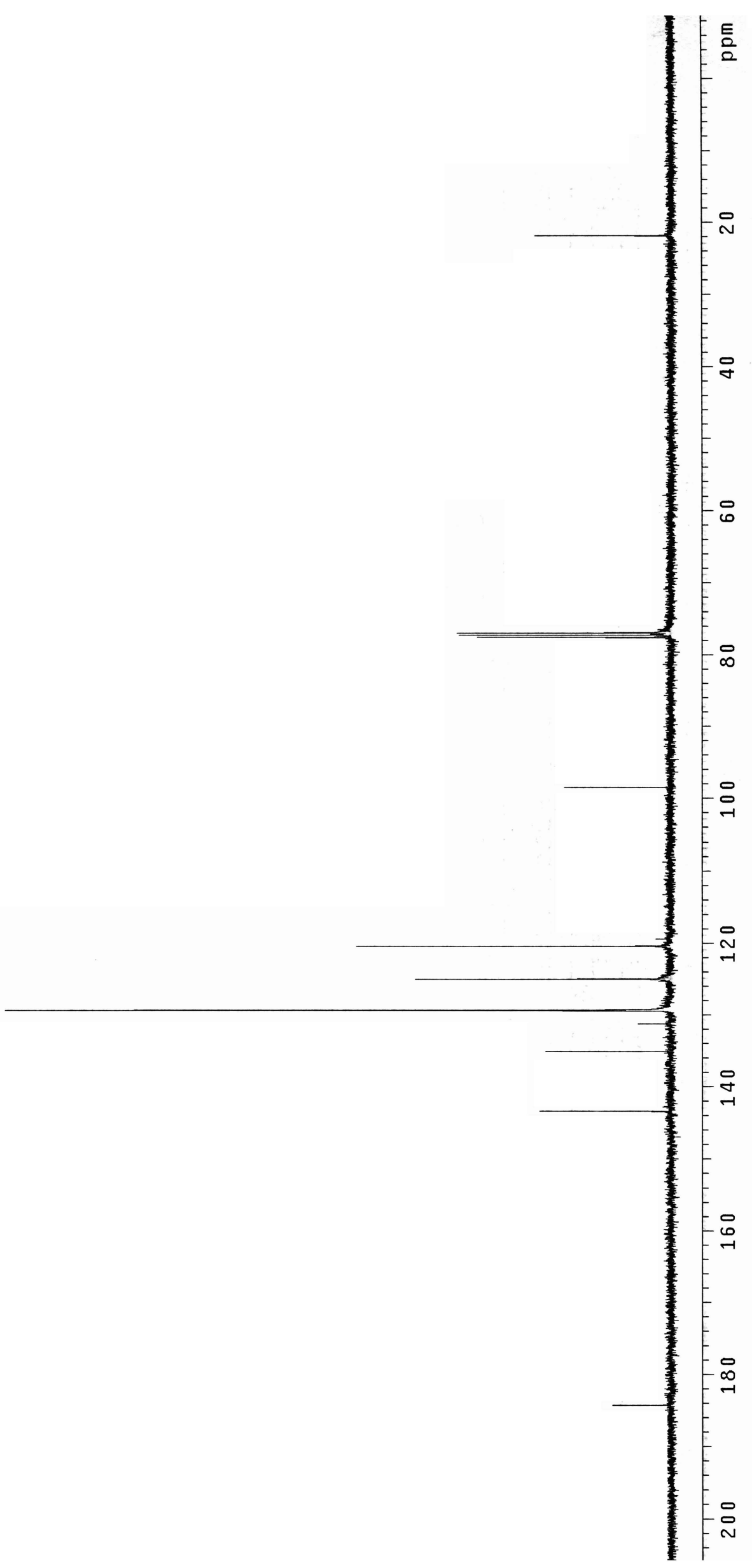




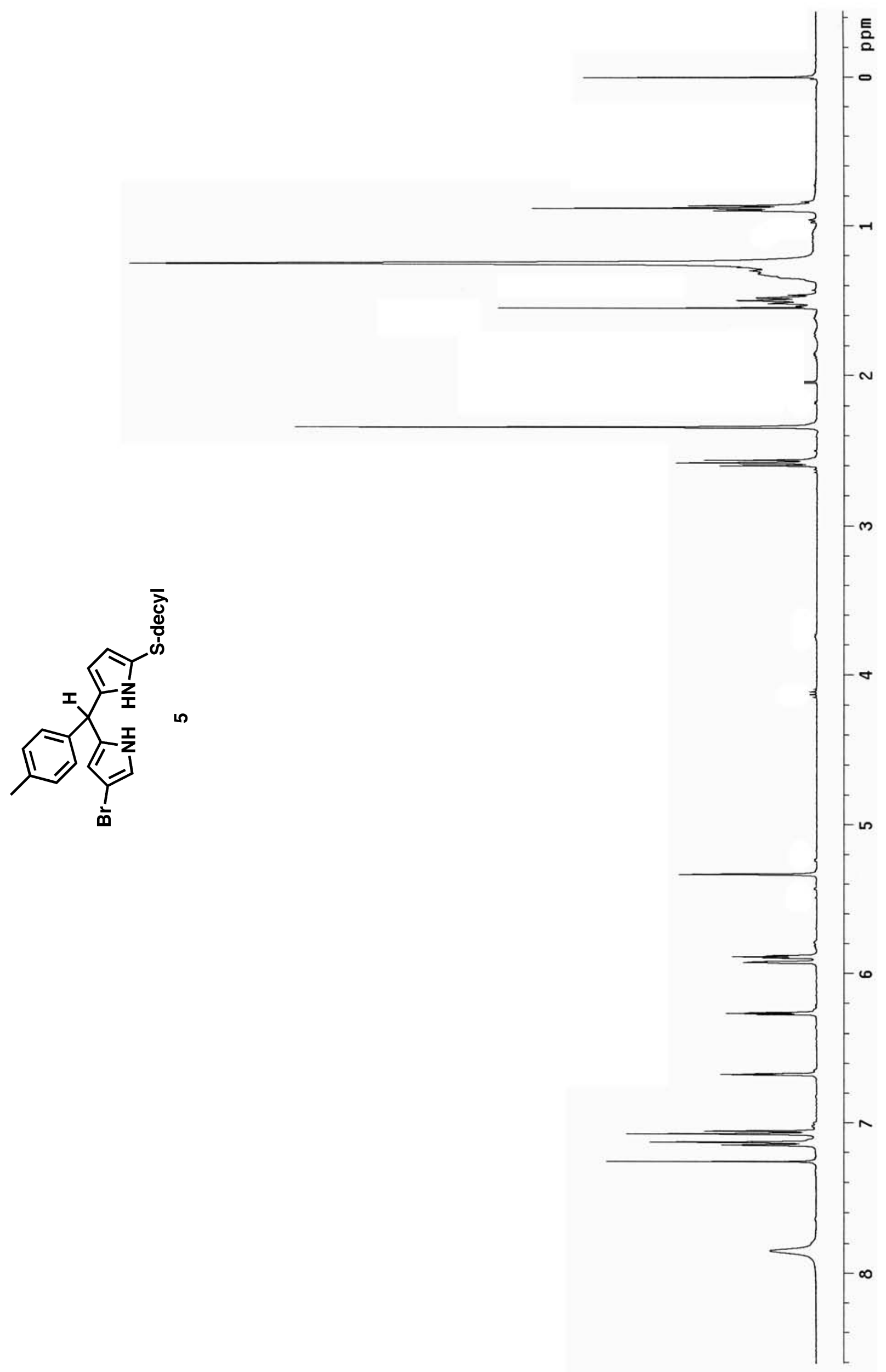

S16 

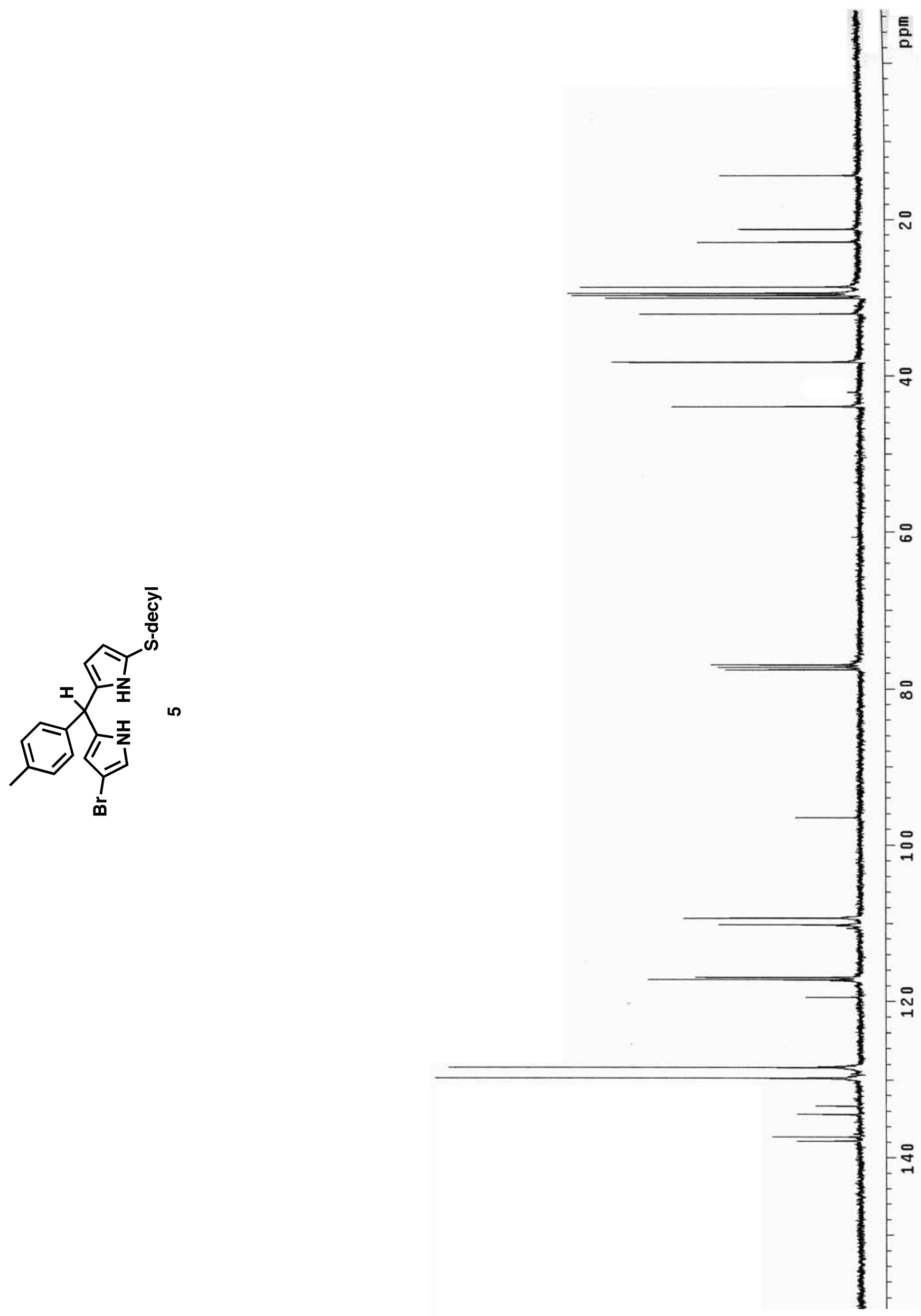


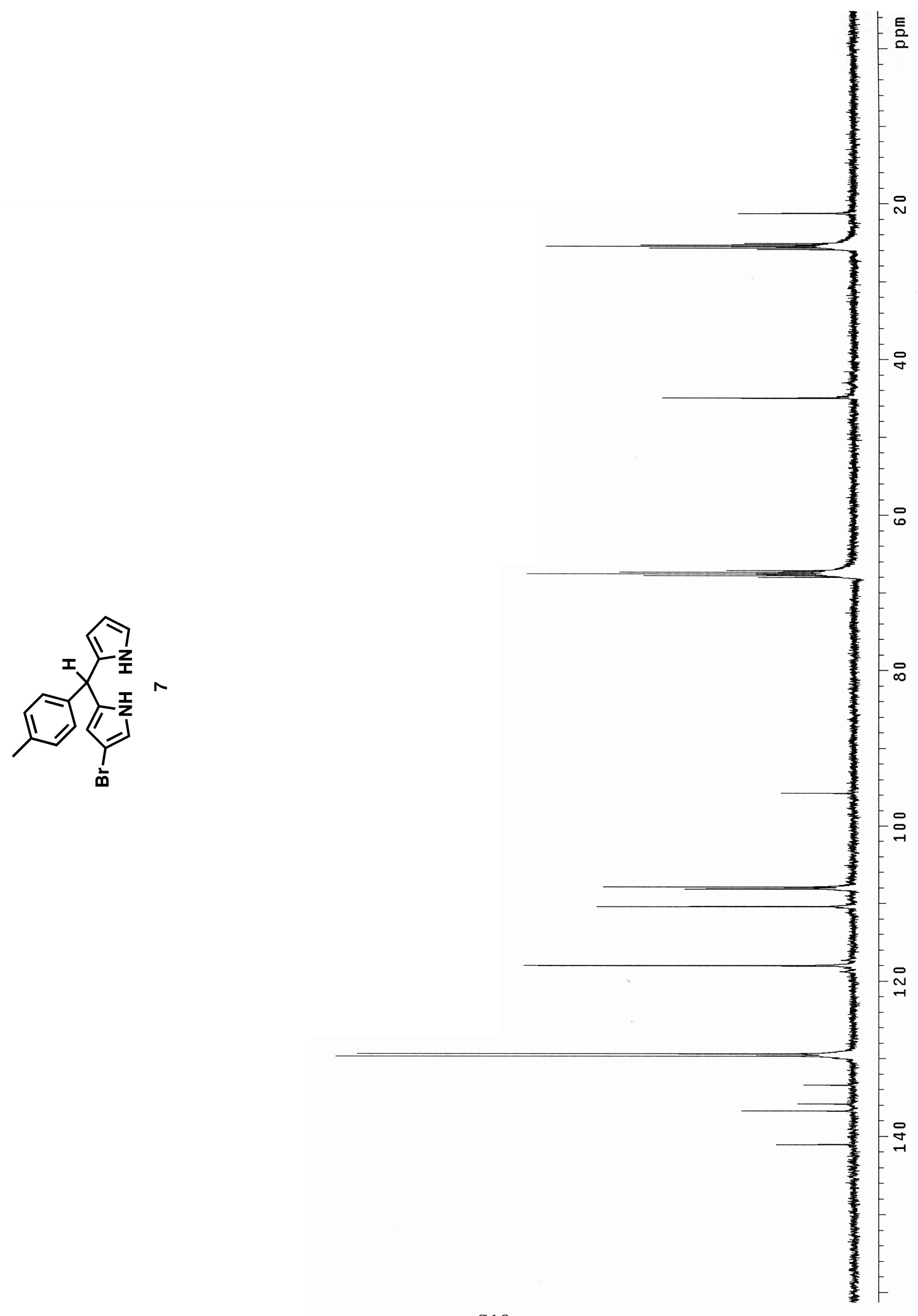




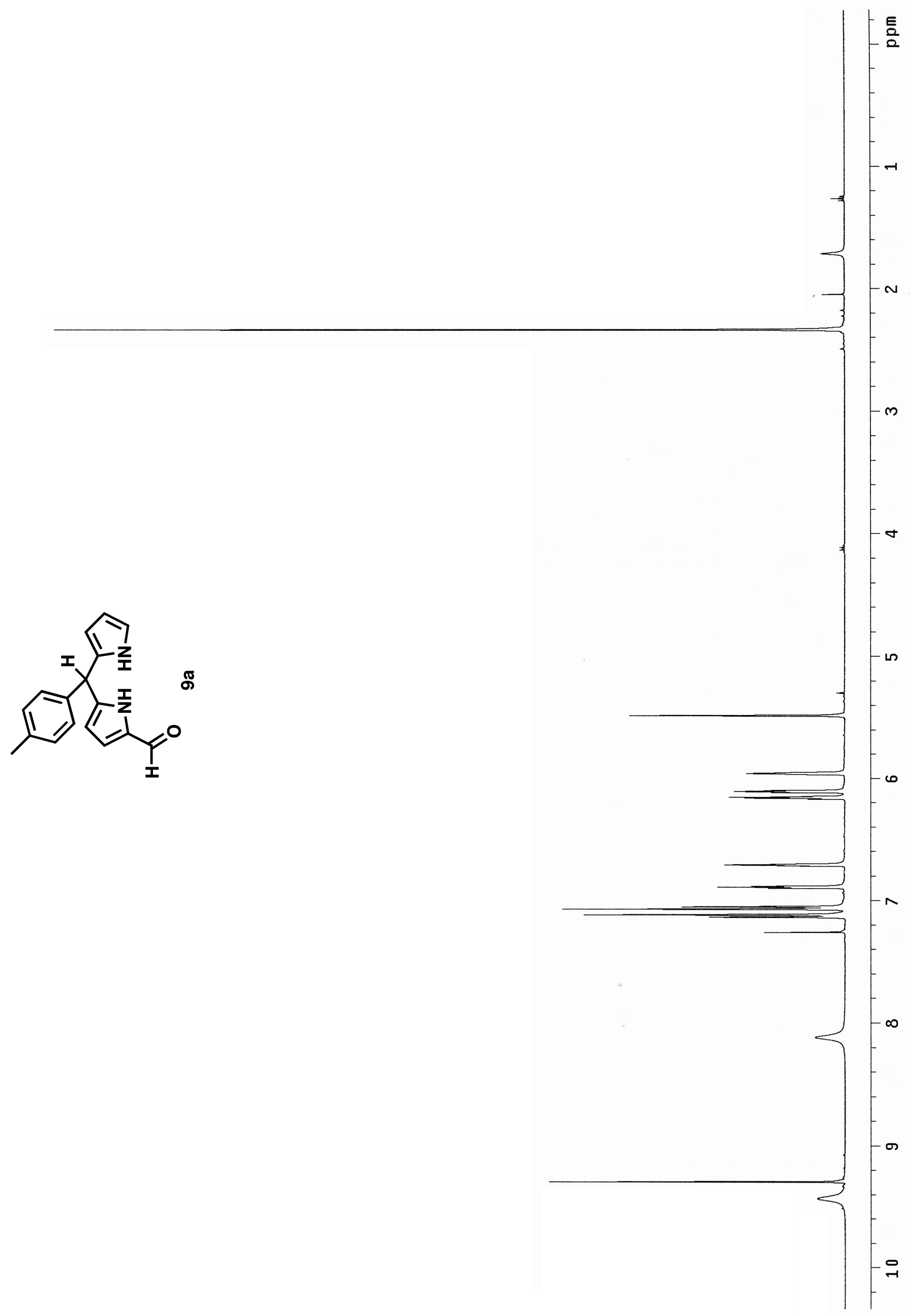




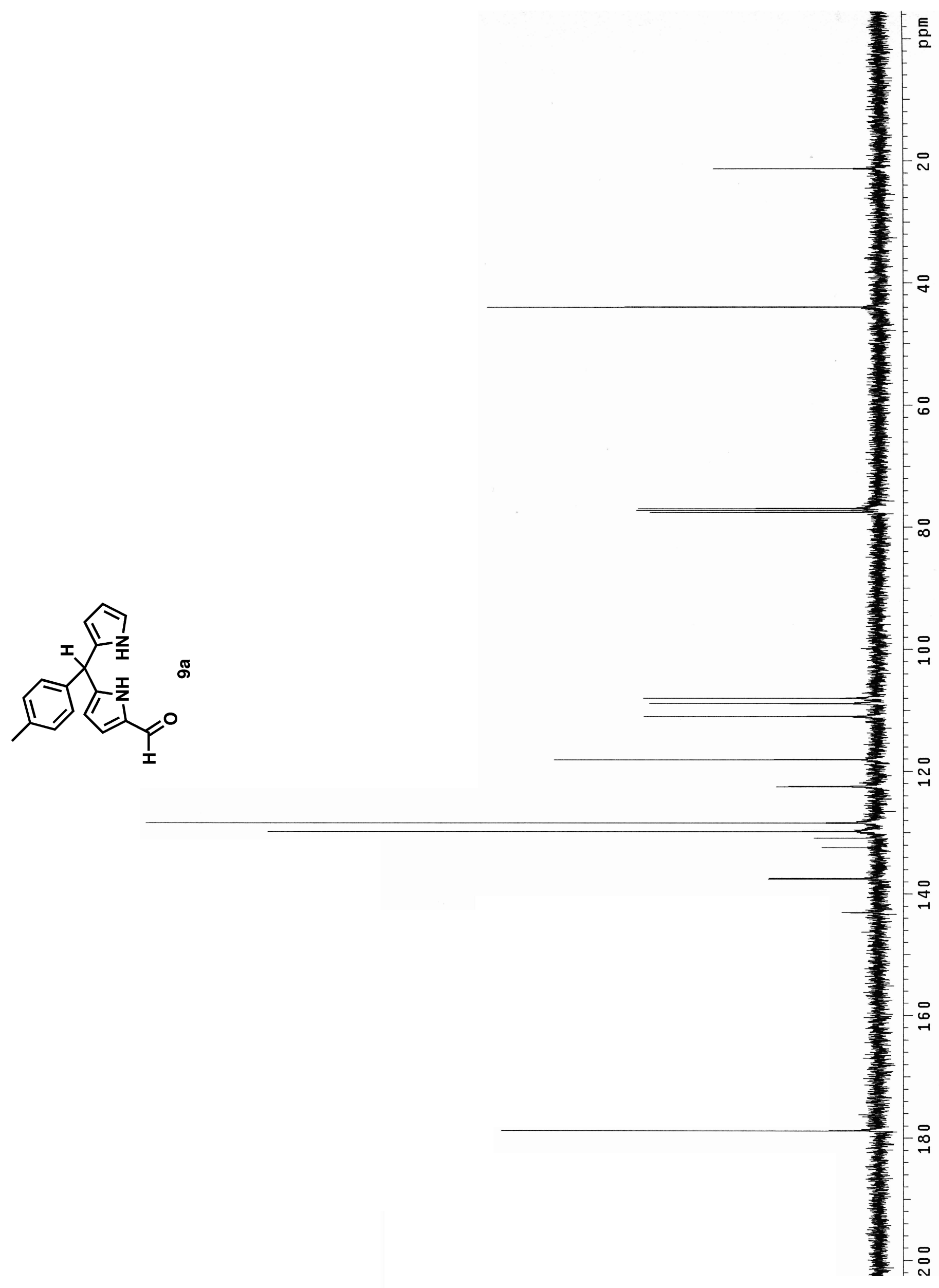




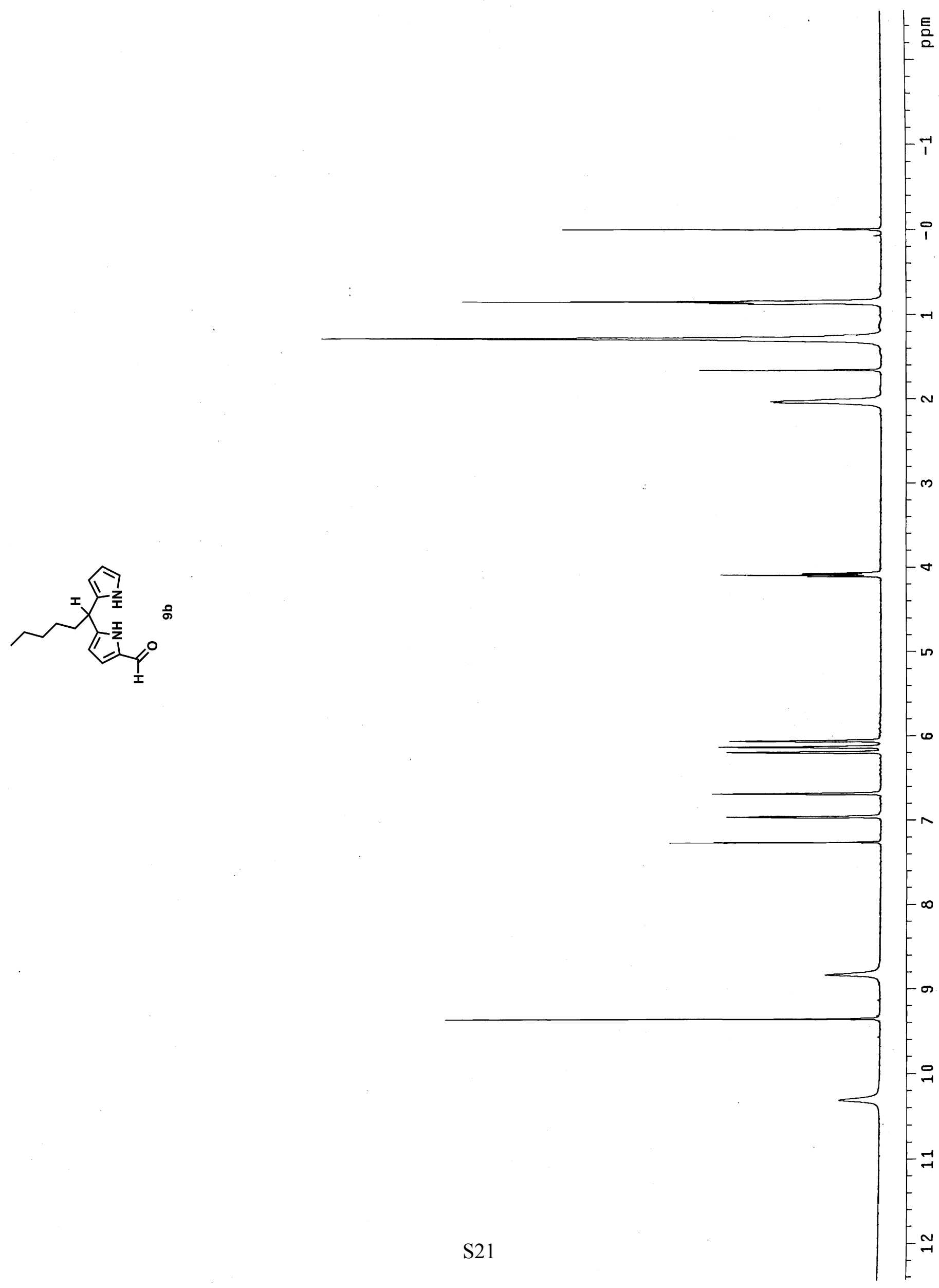




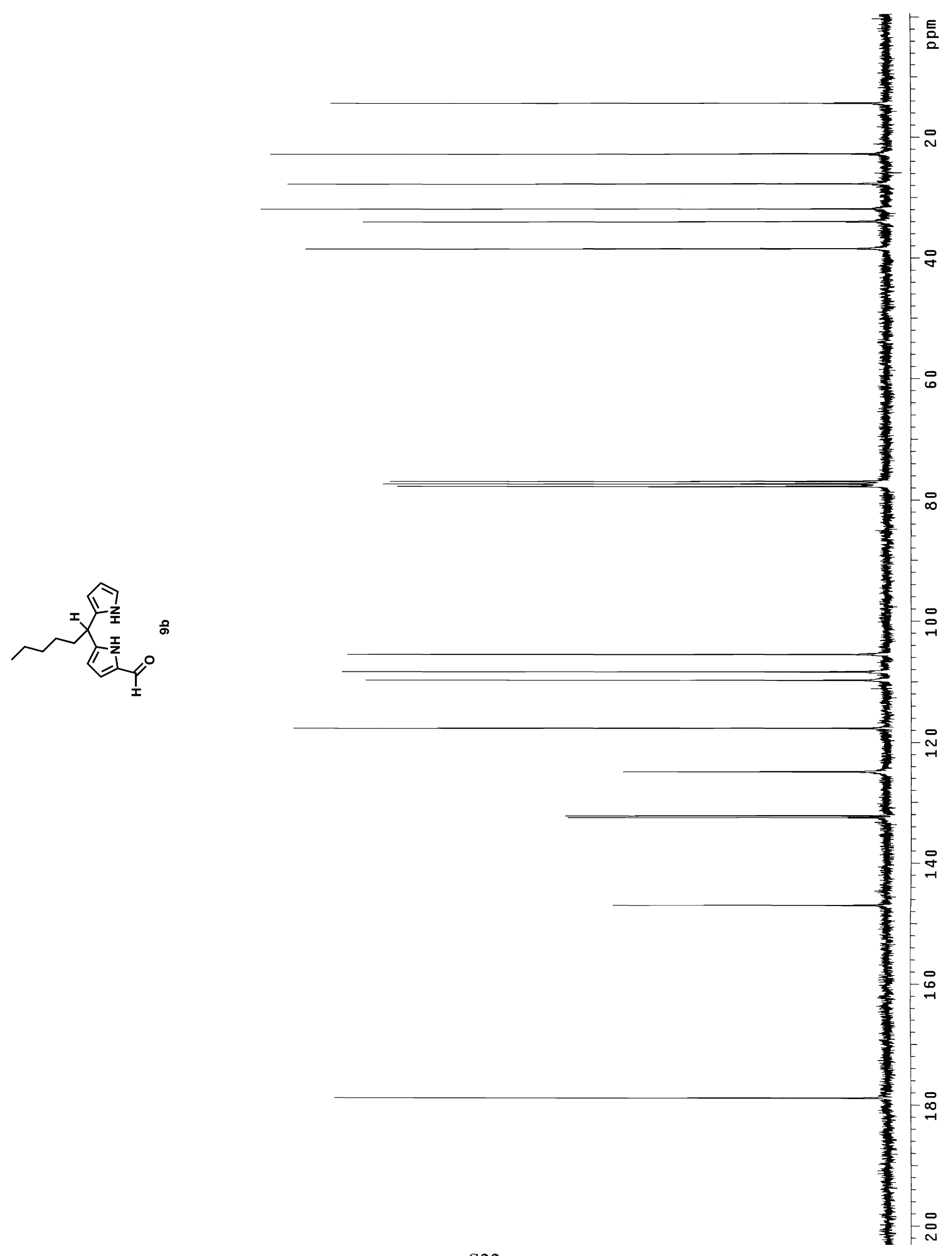




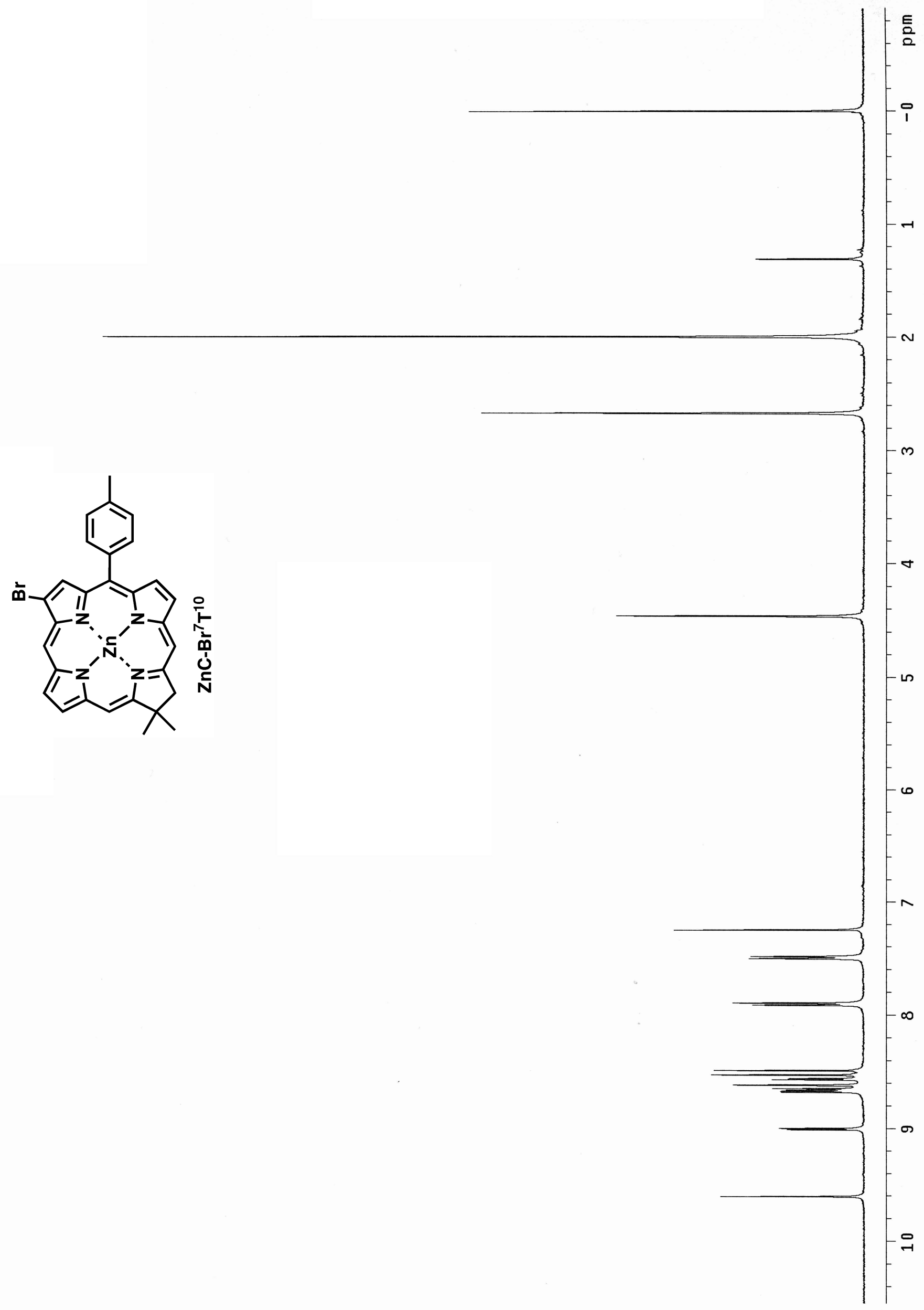




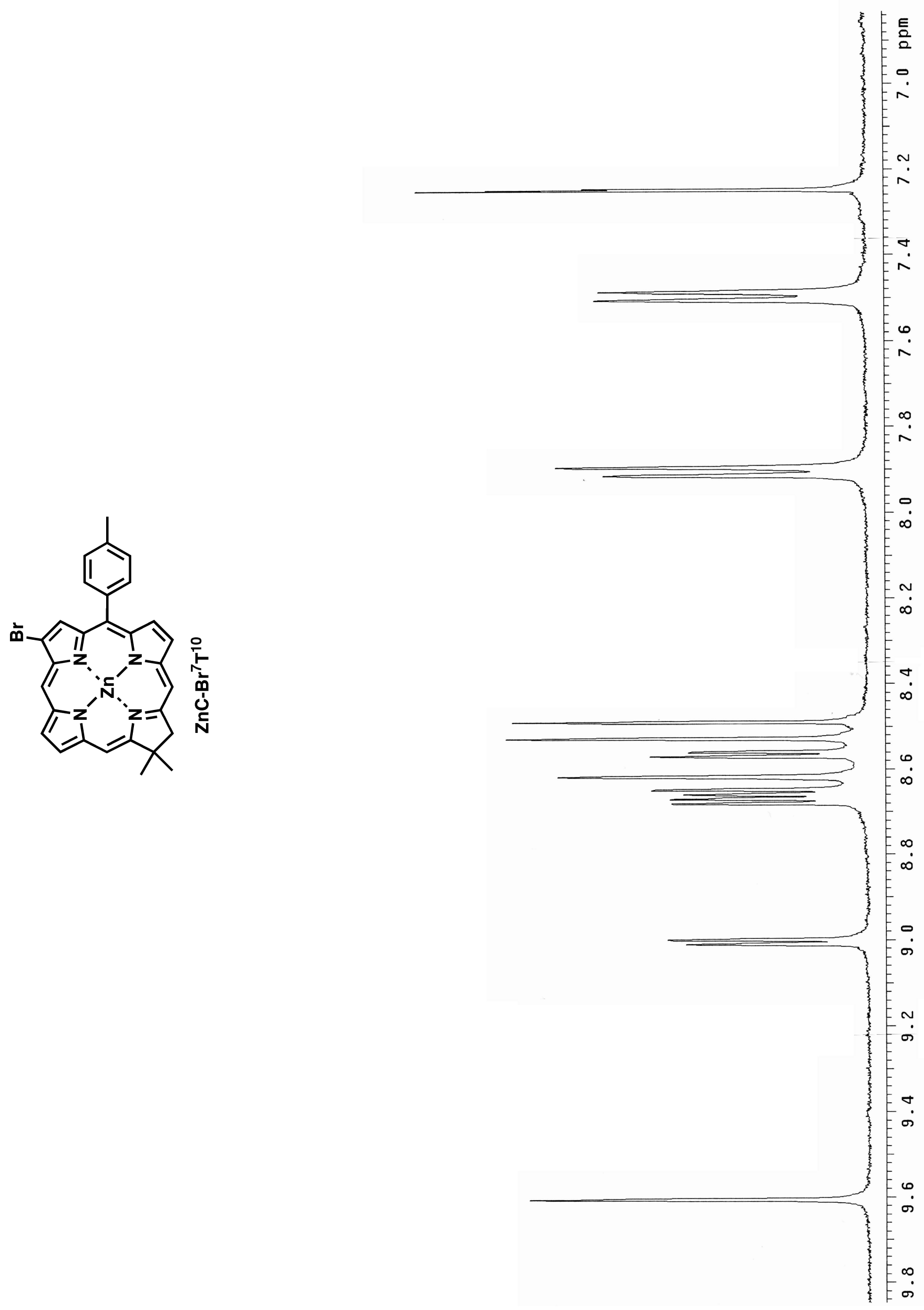




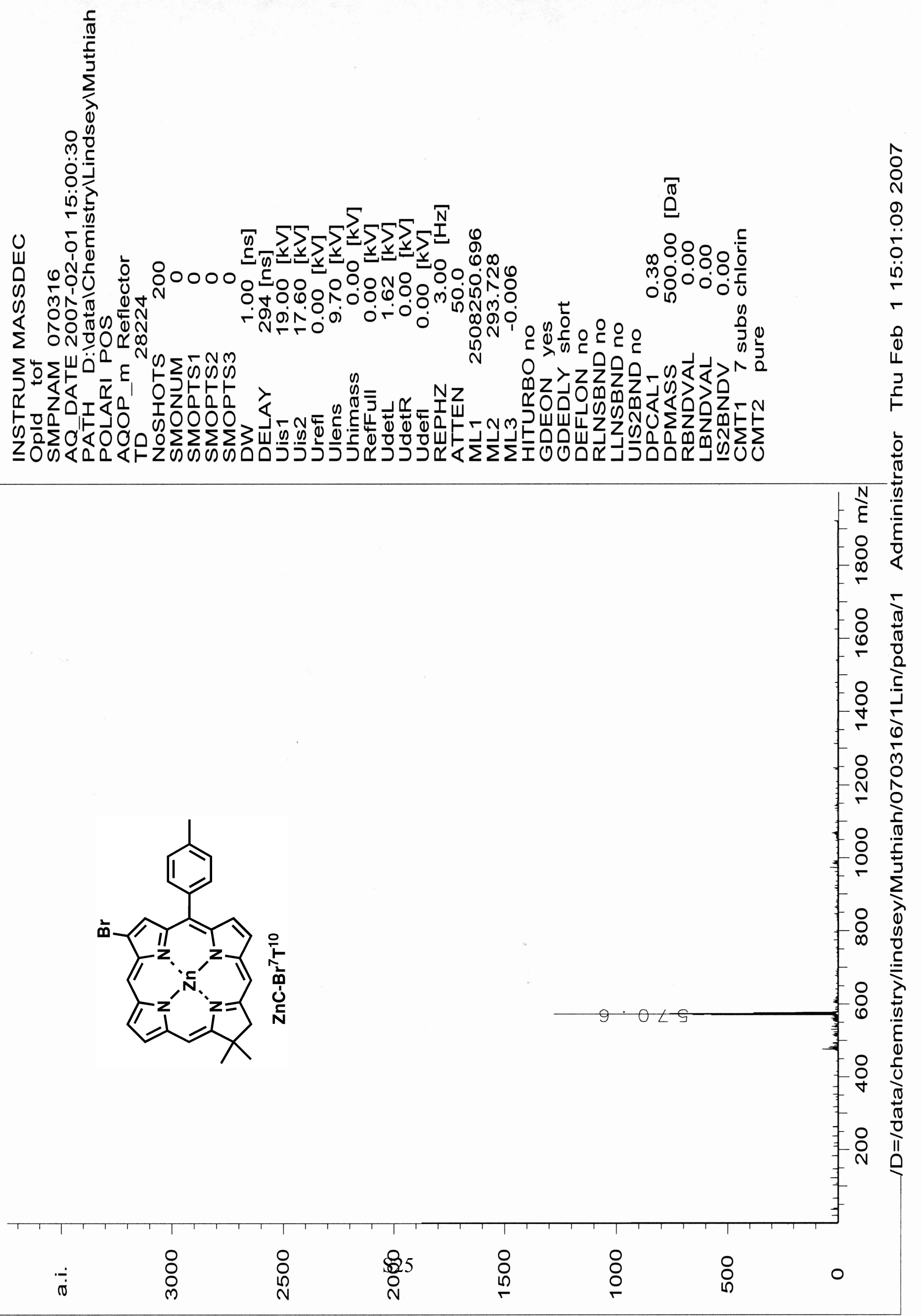




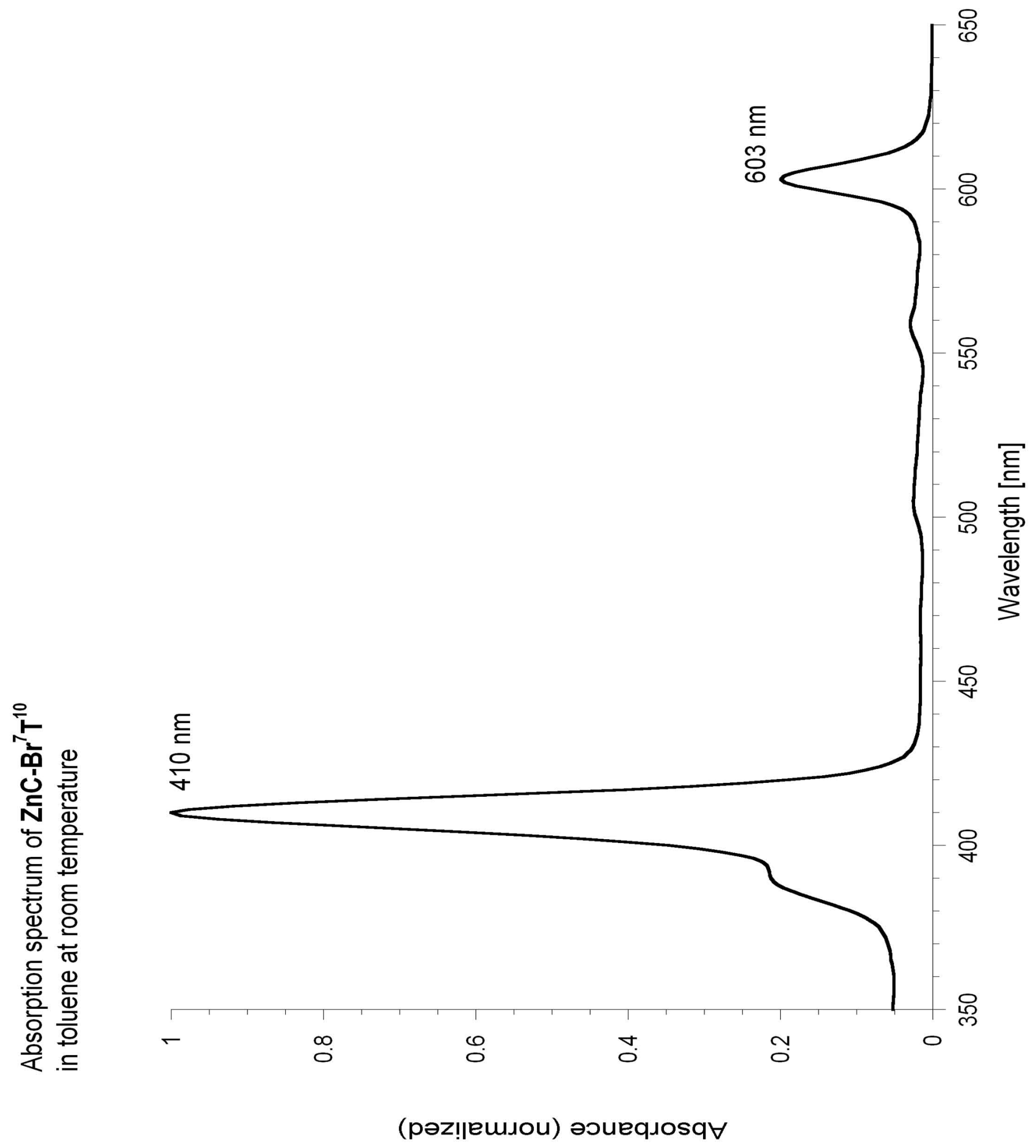




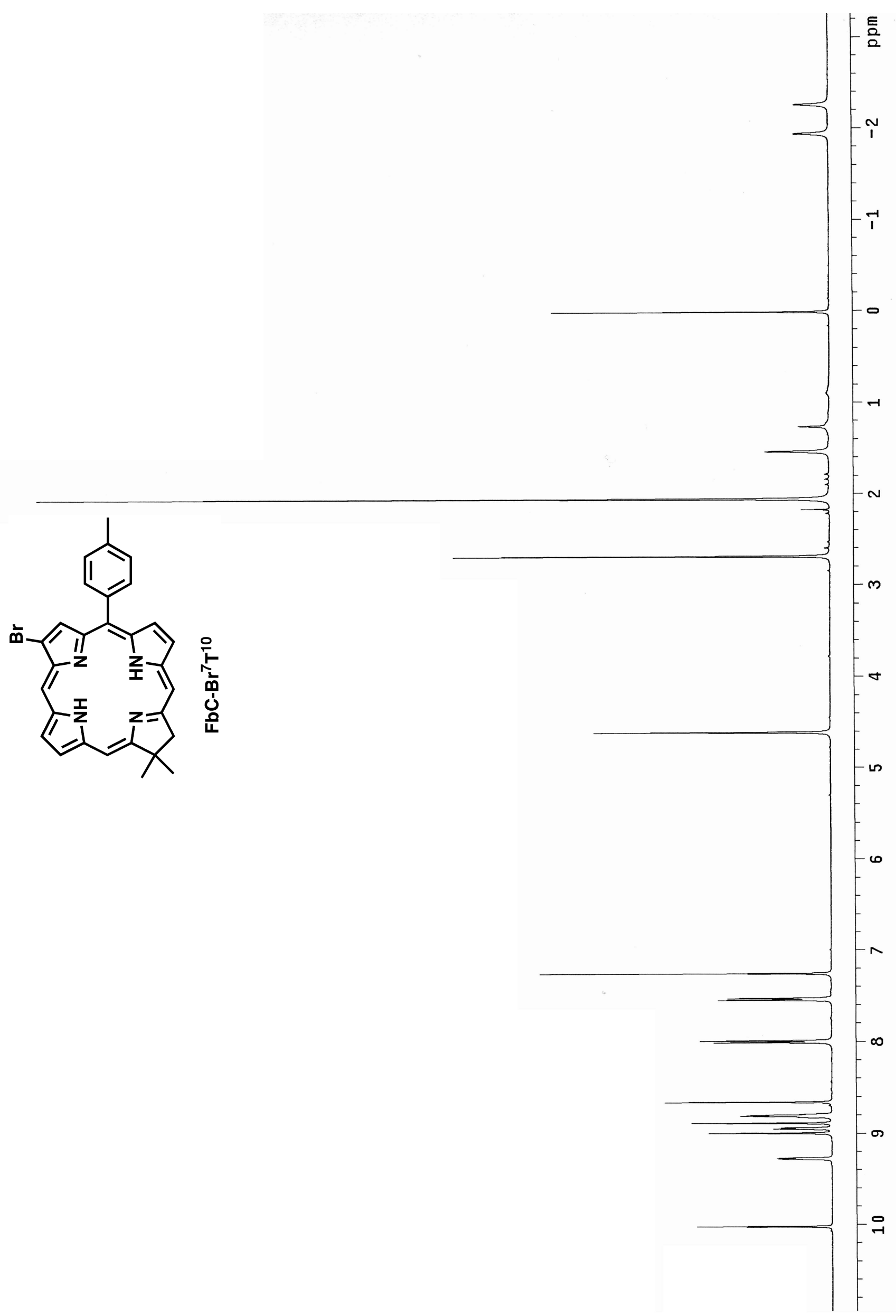




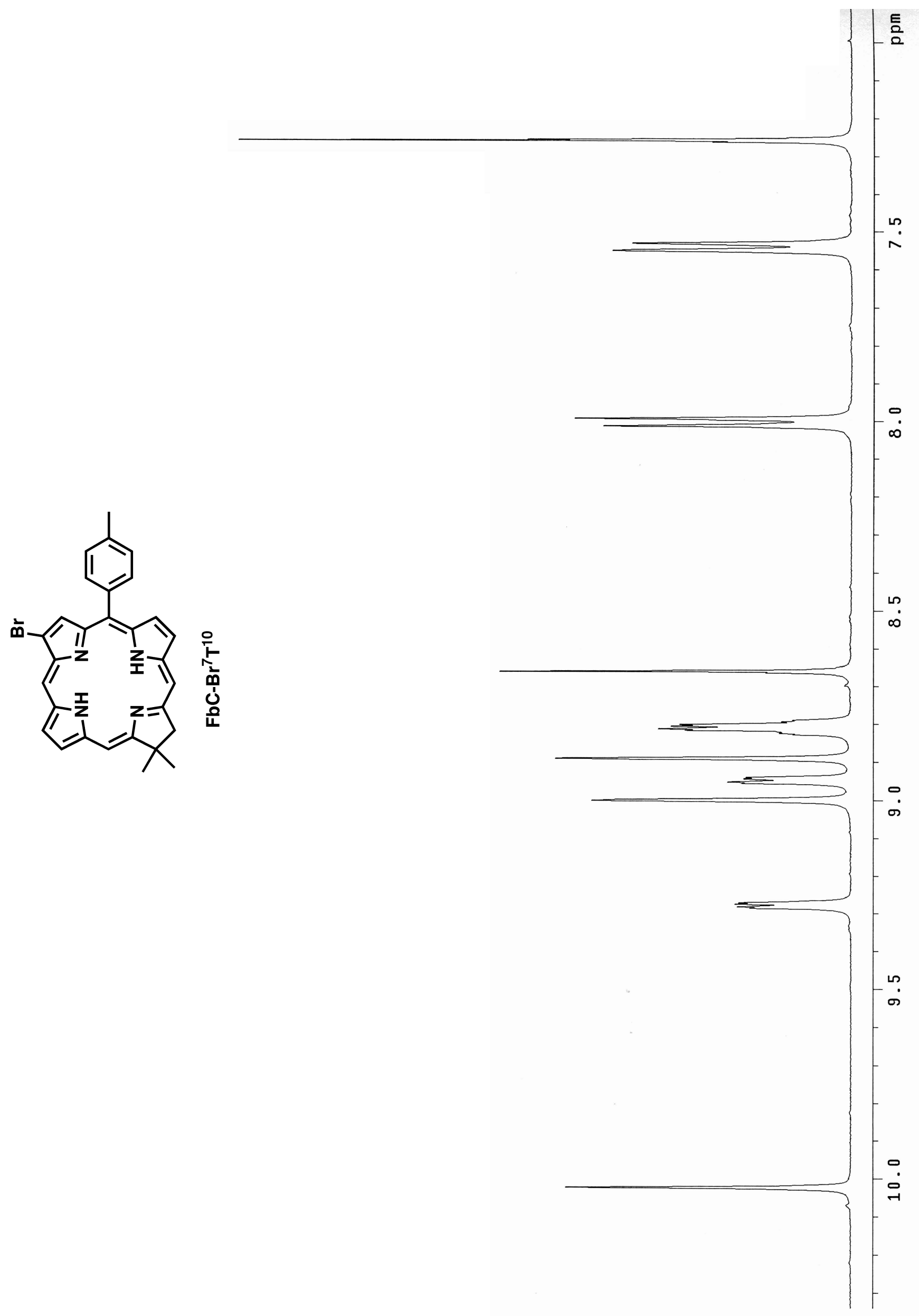




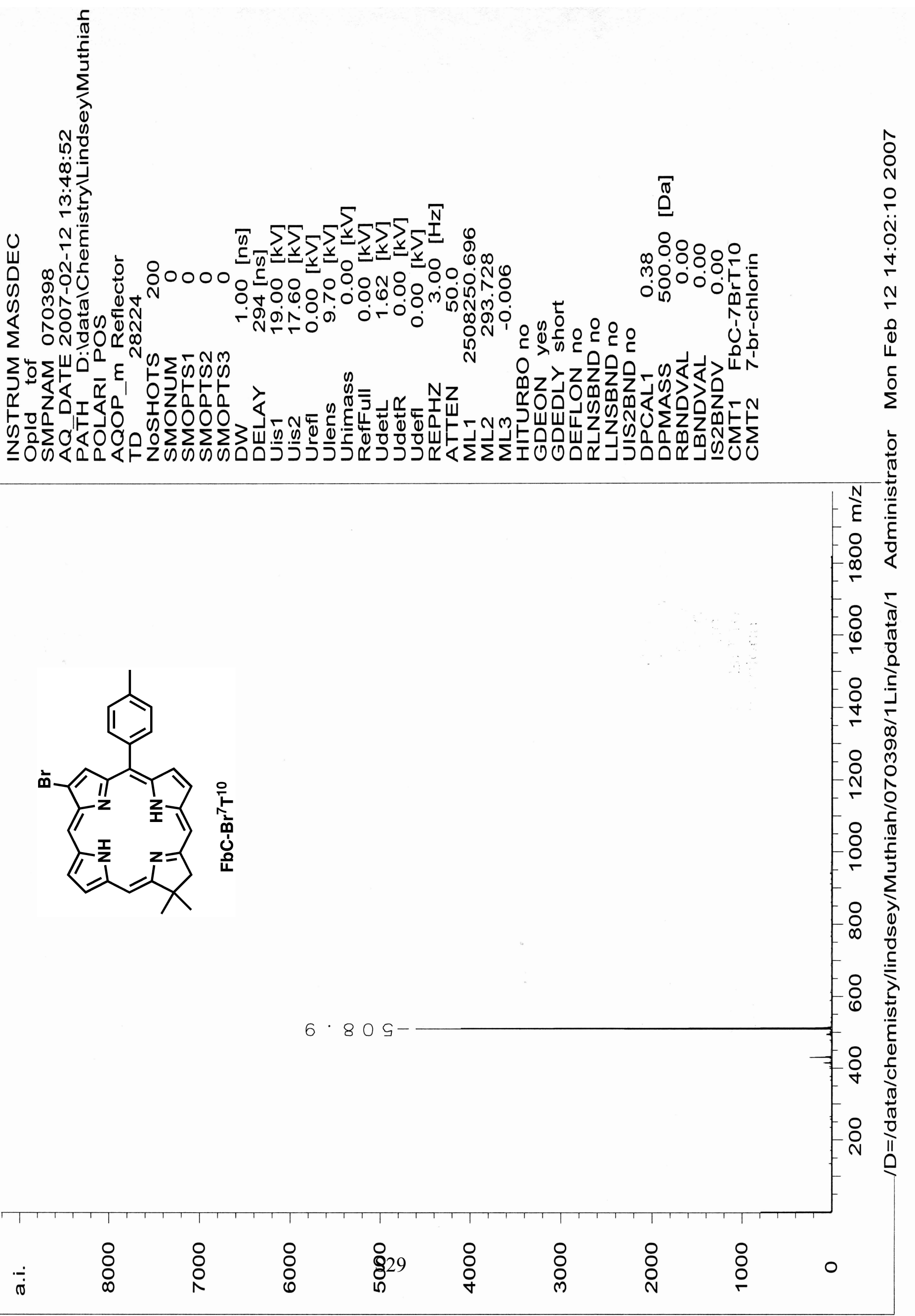




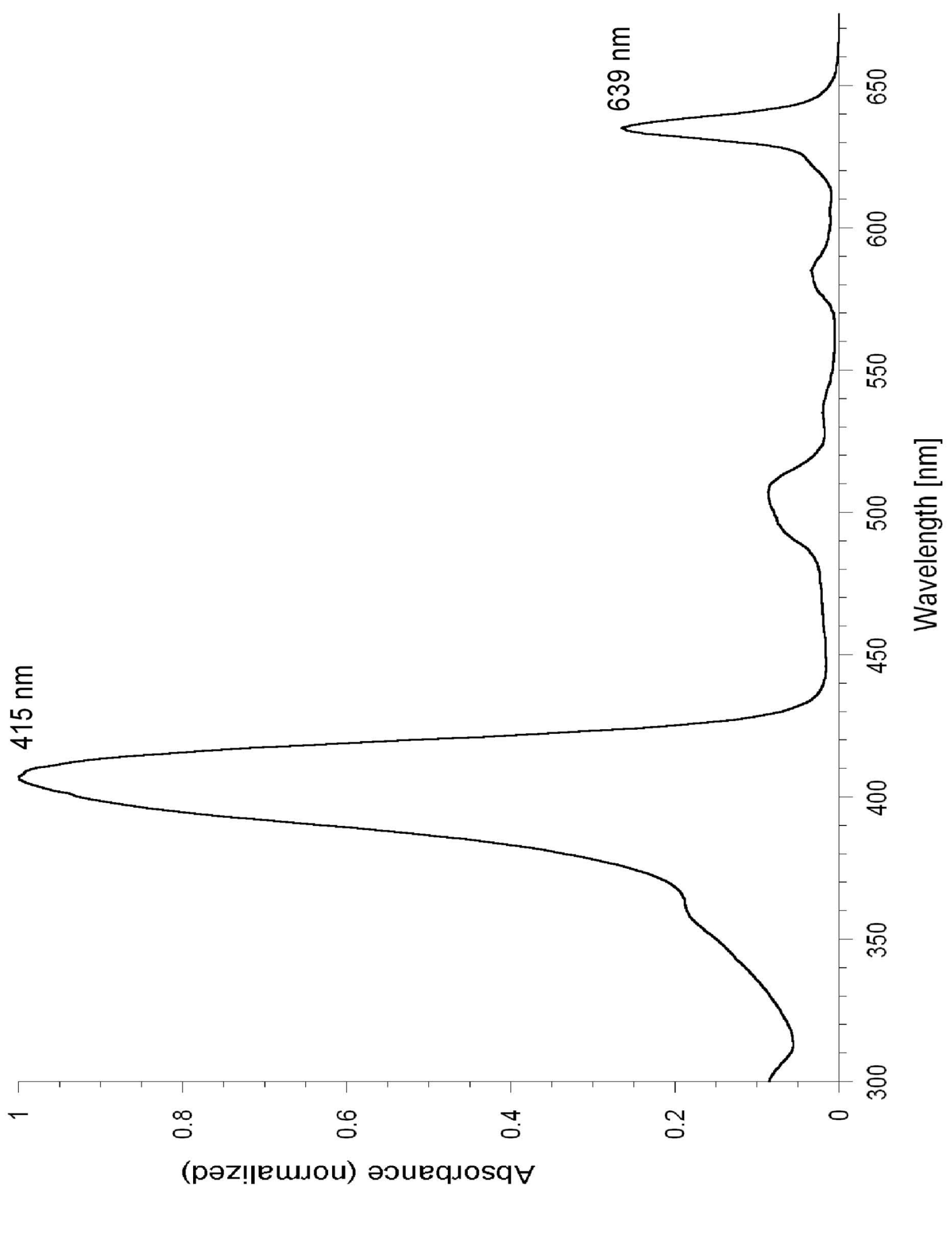




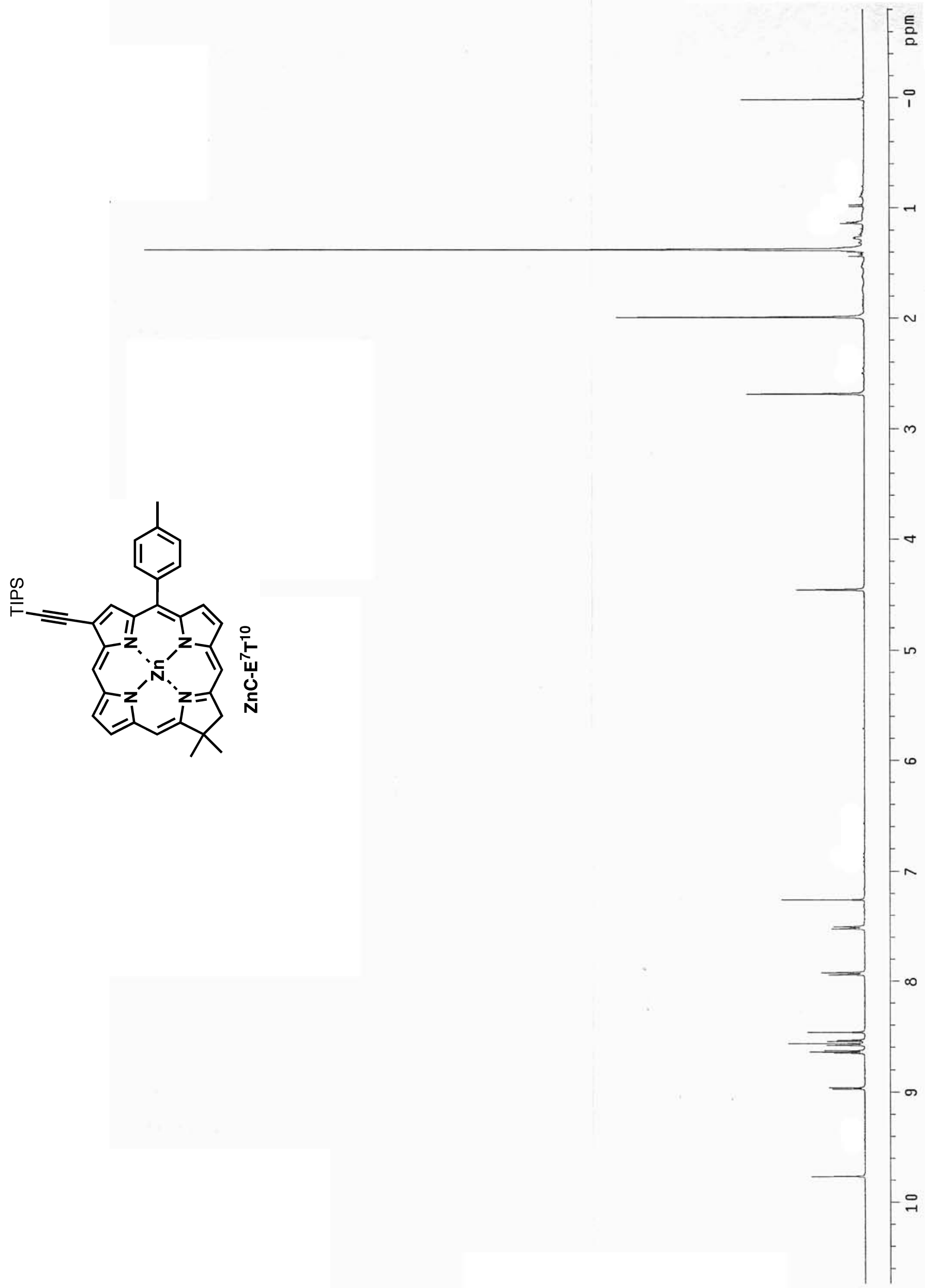




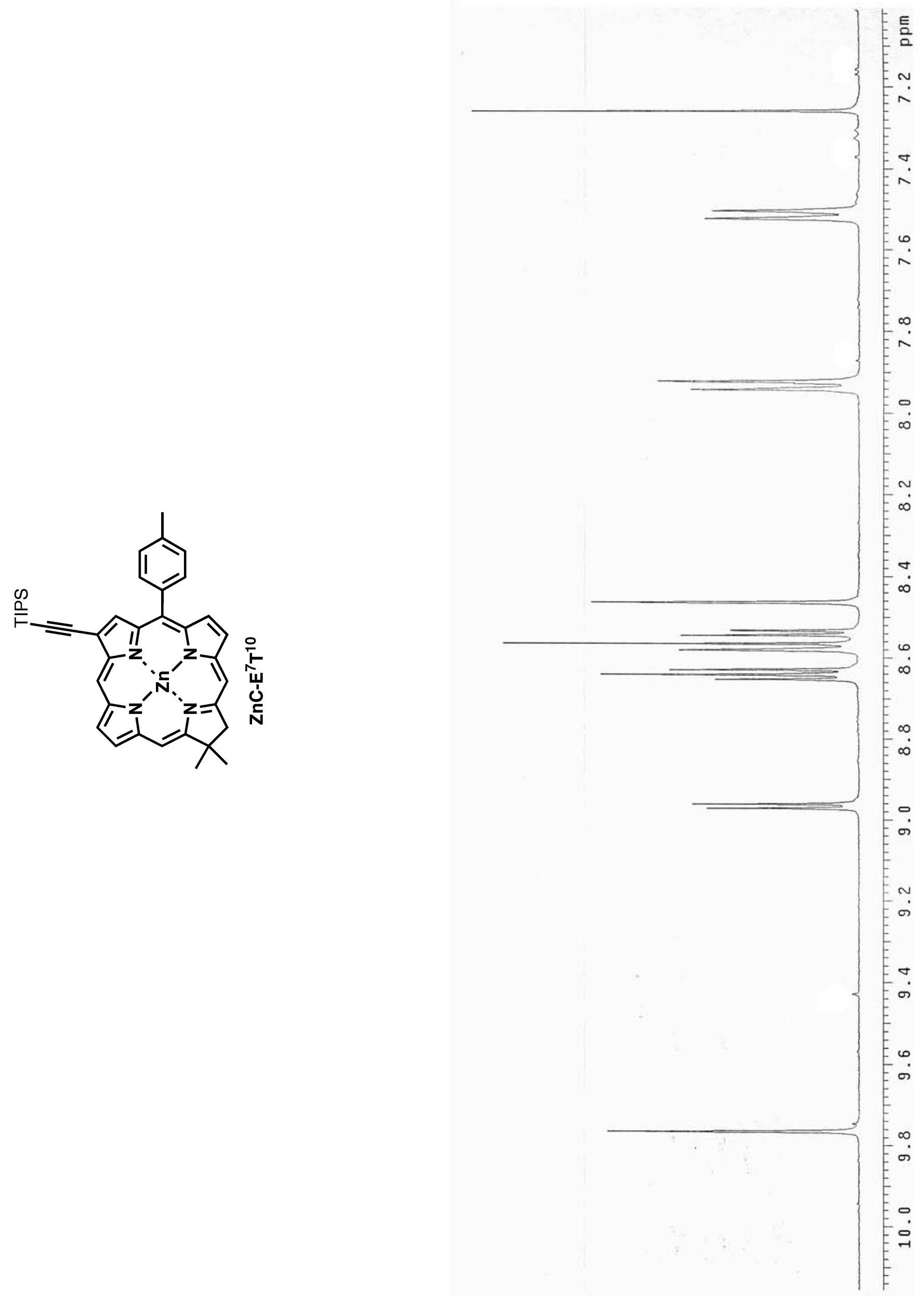




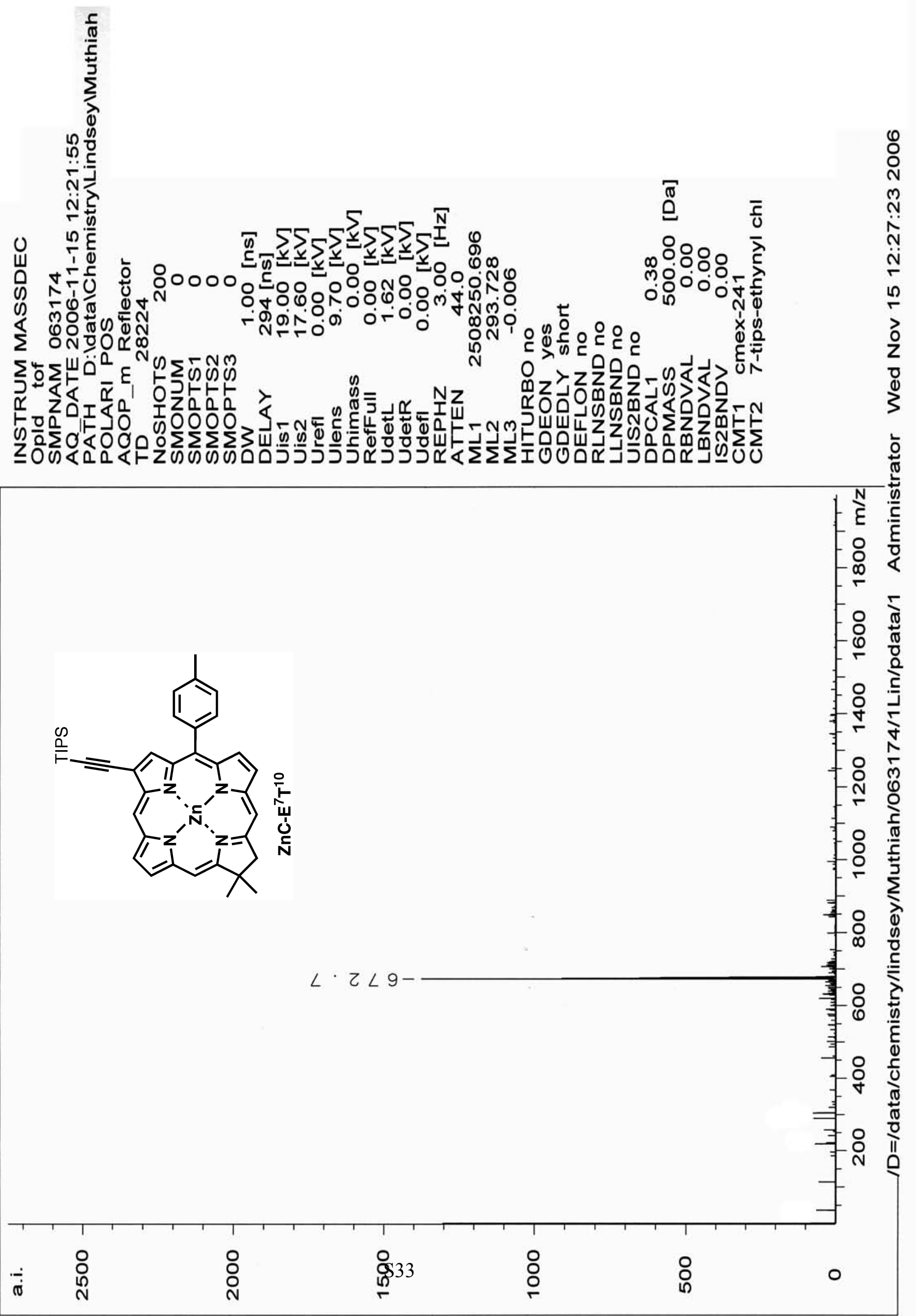




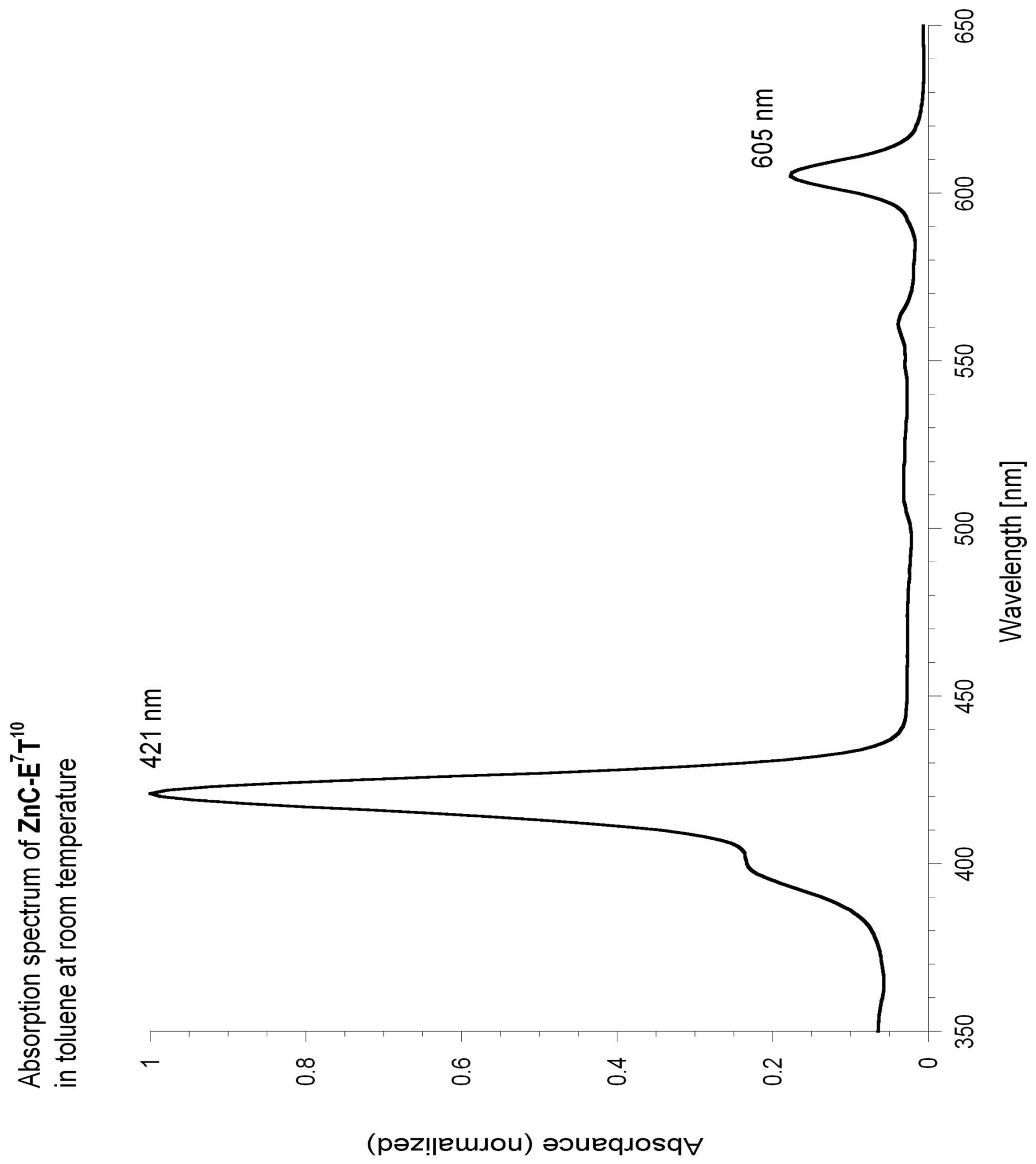




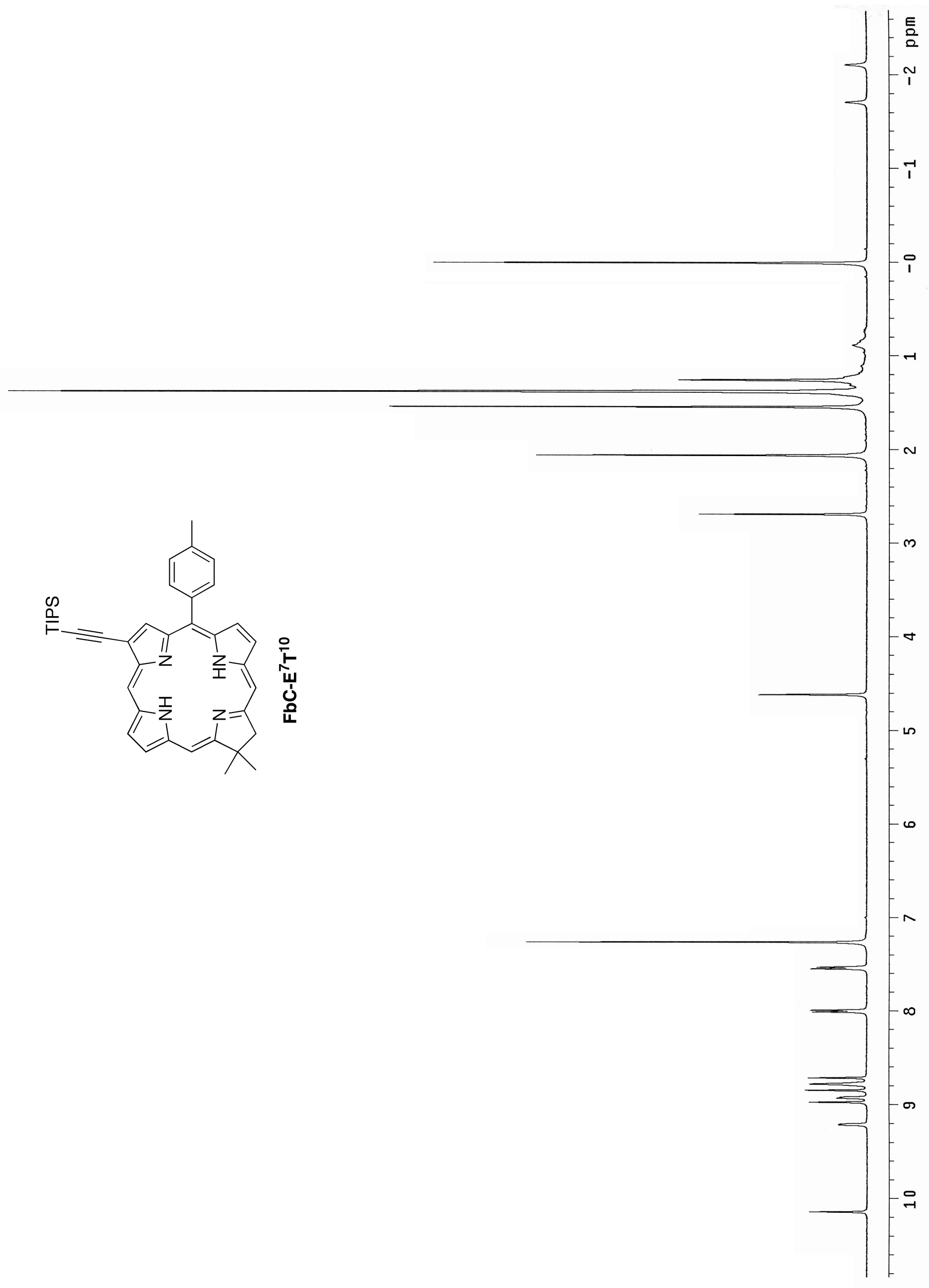




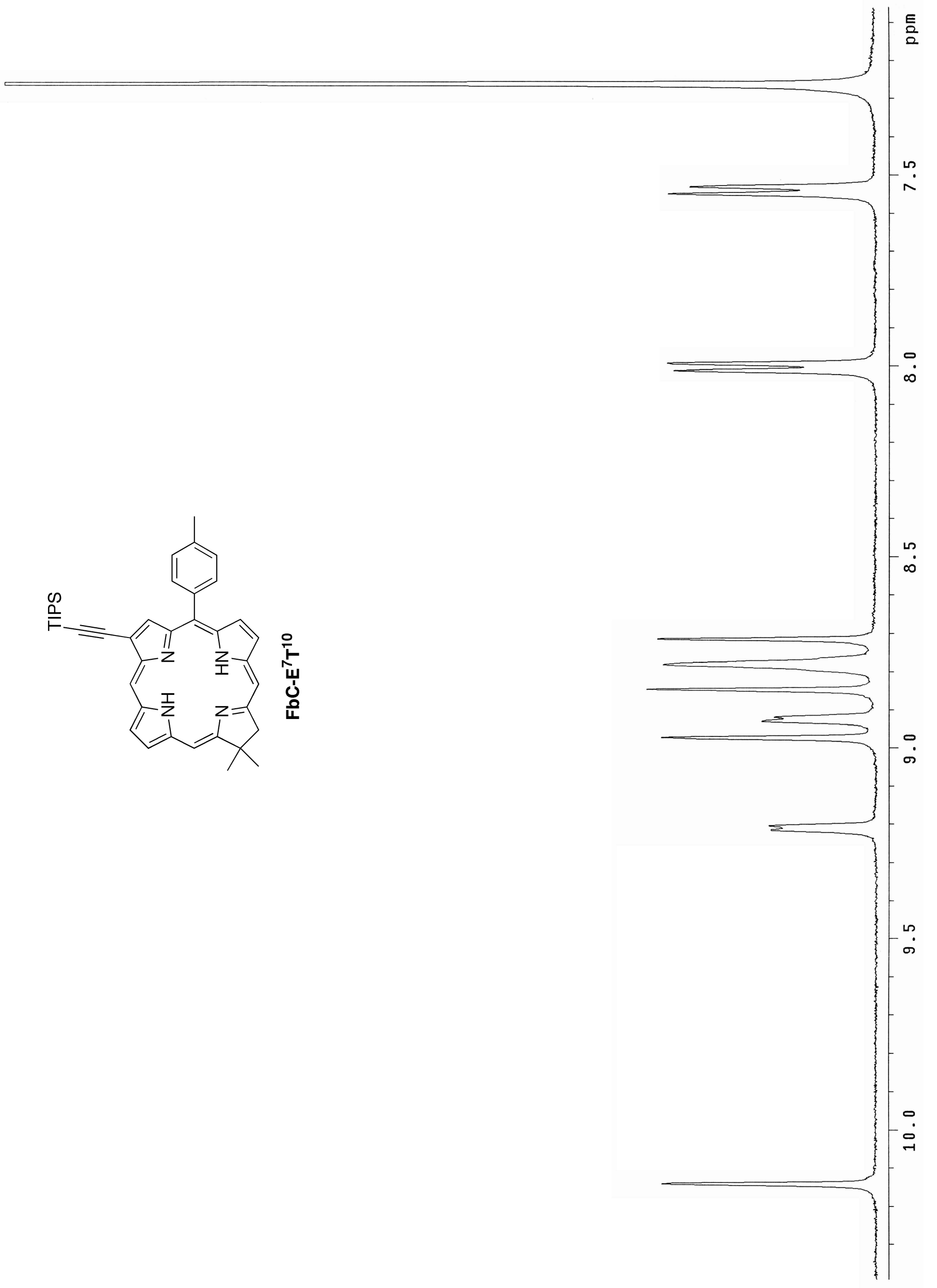




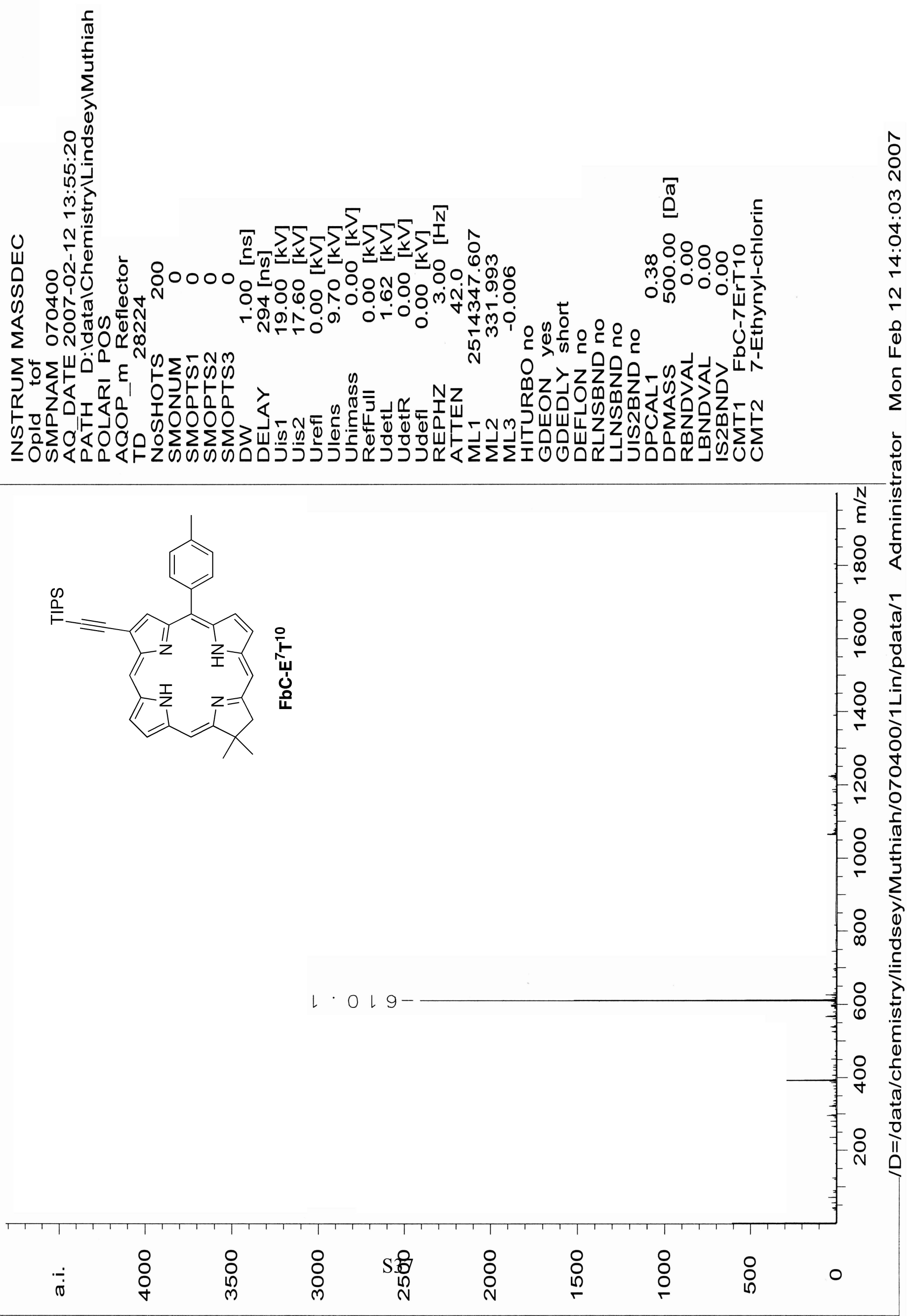




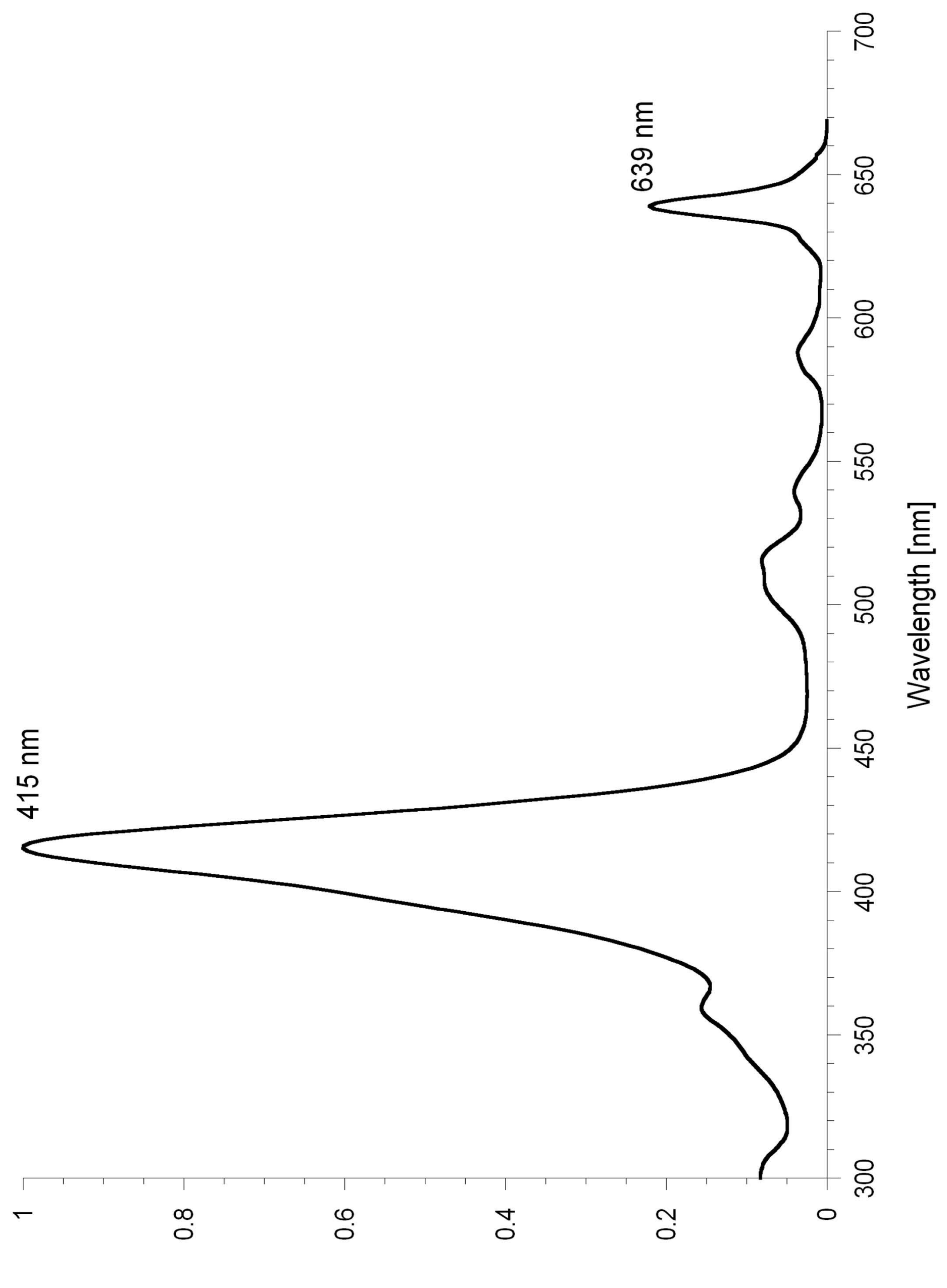




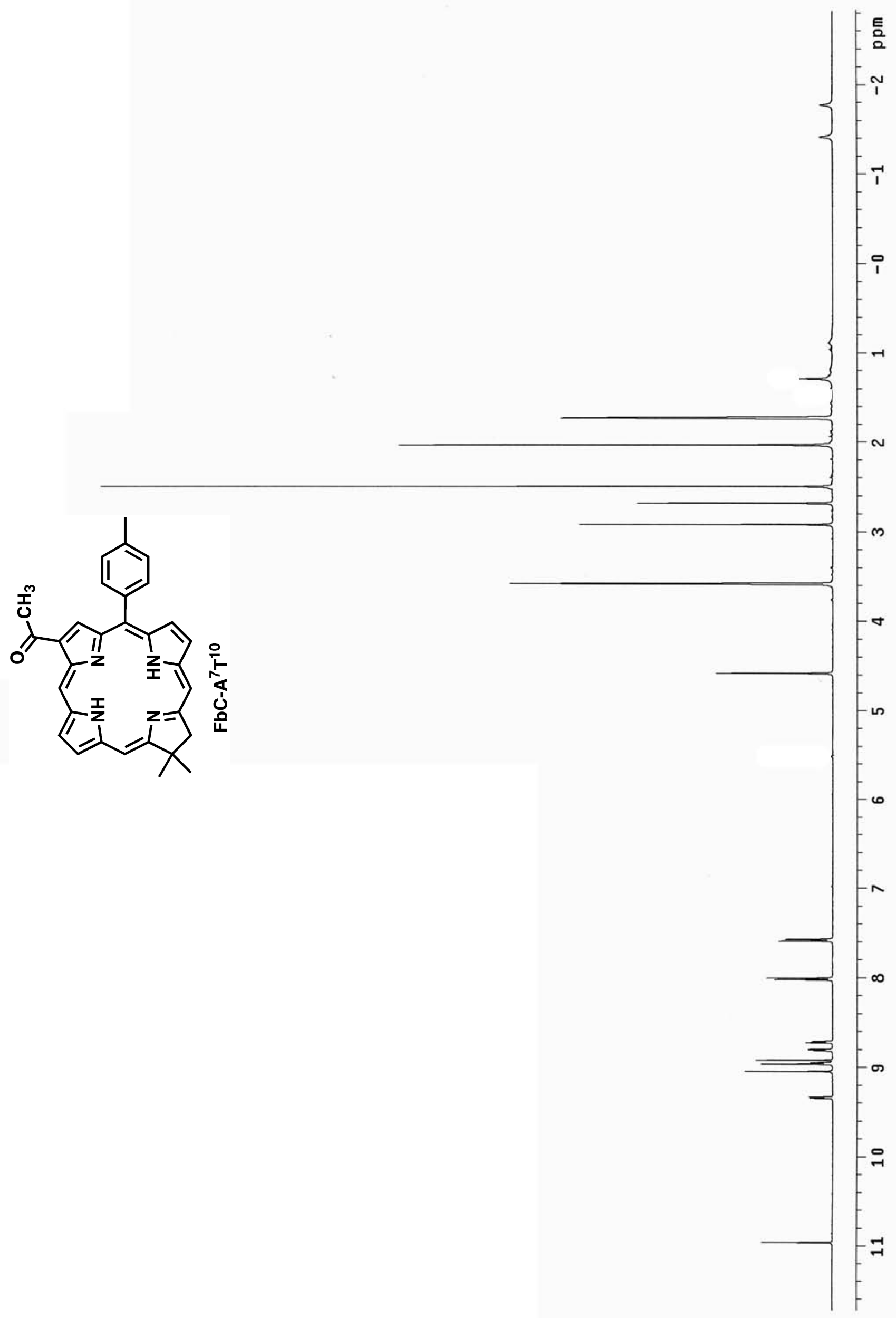




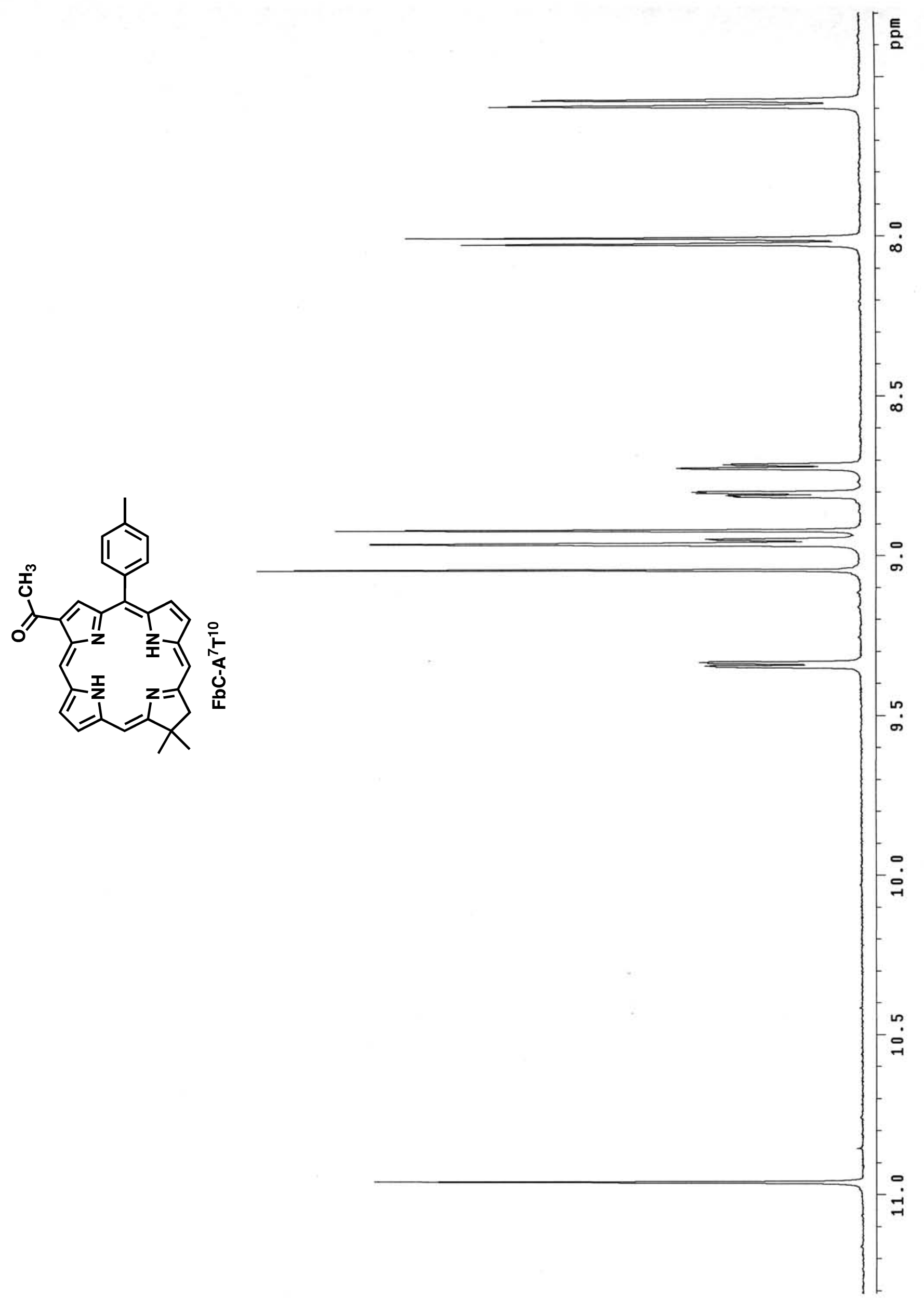




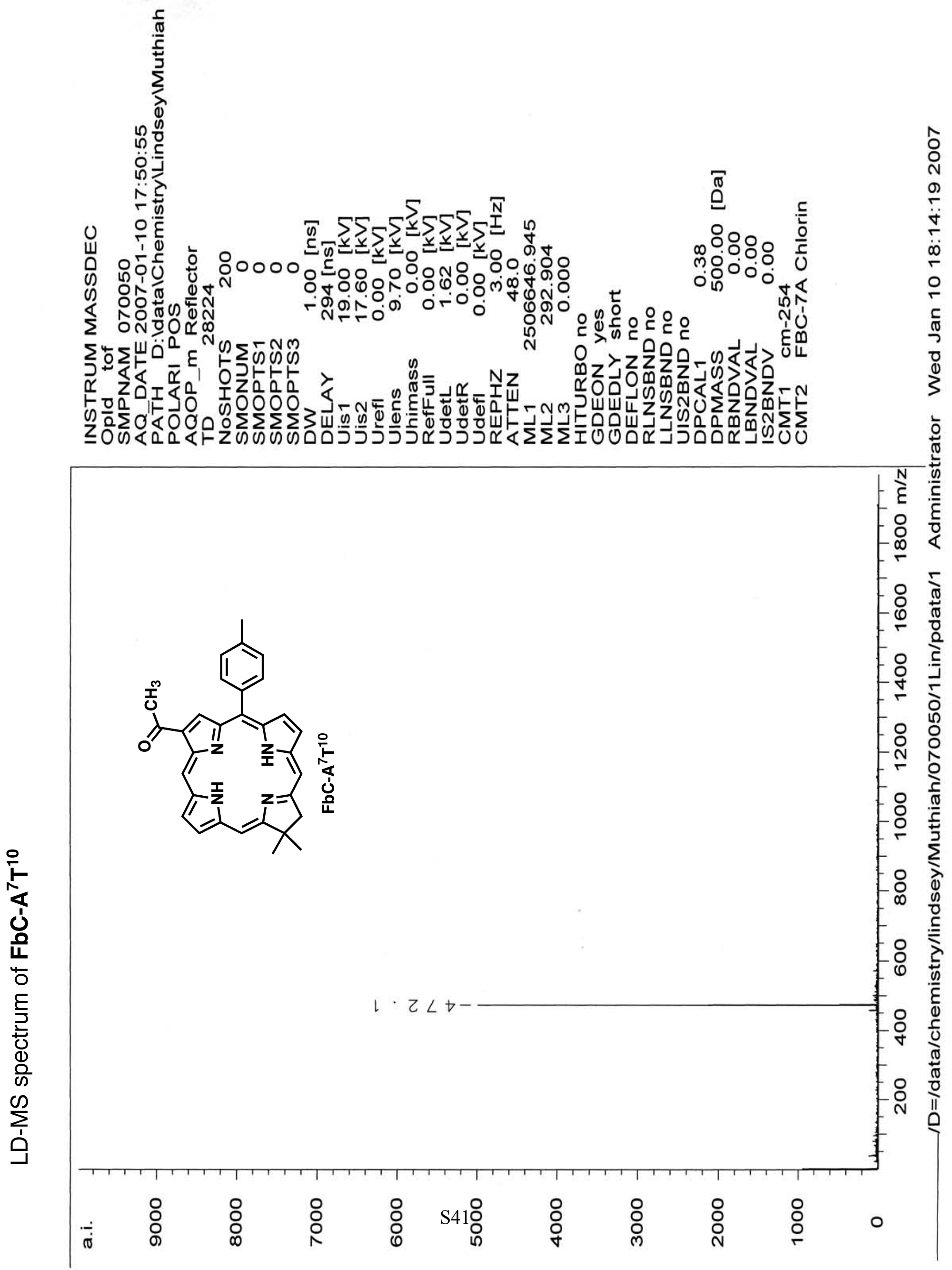




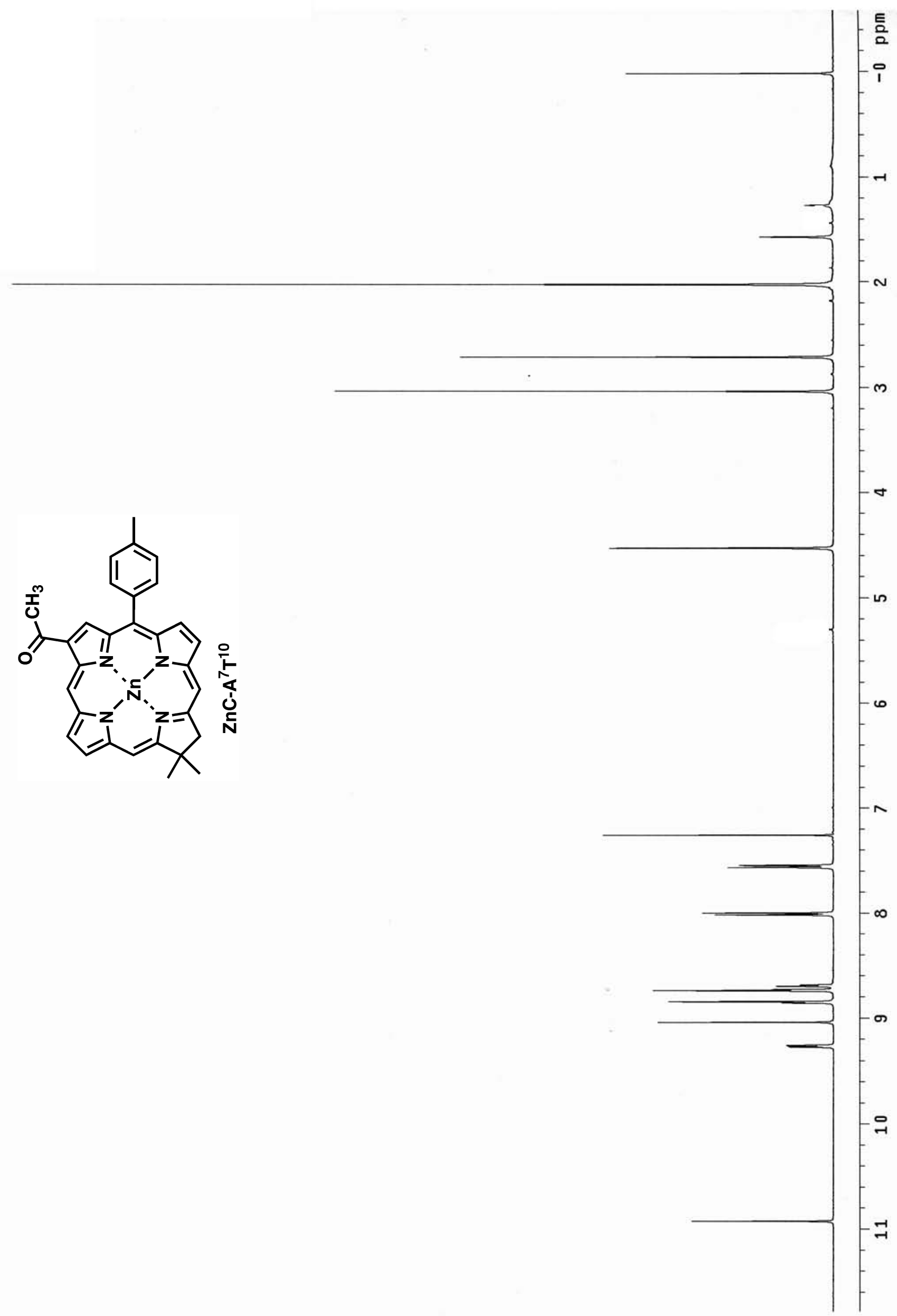




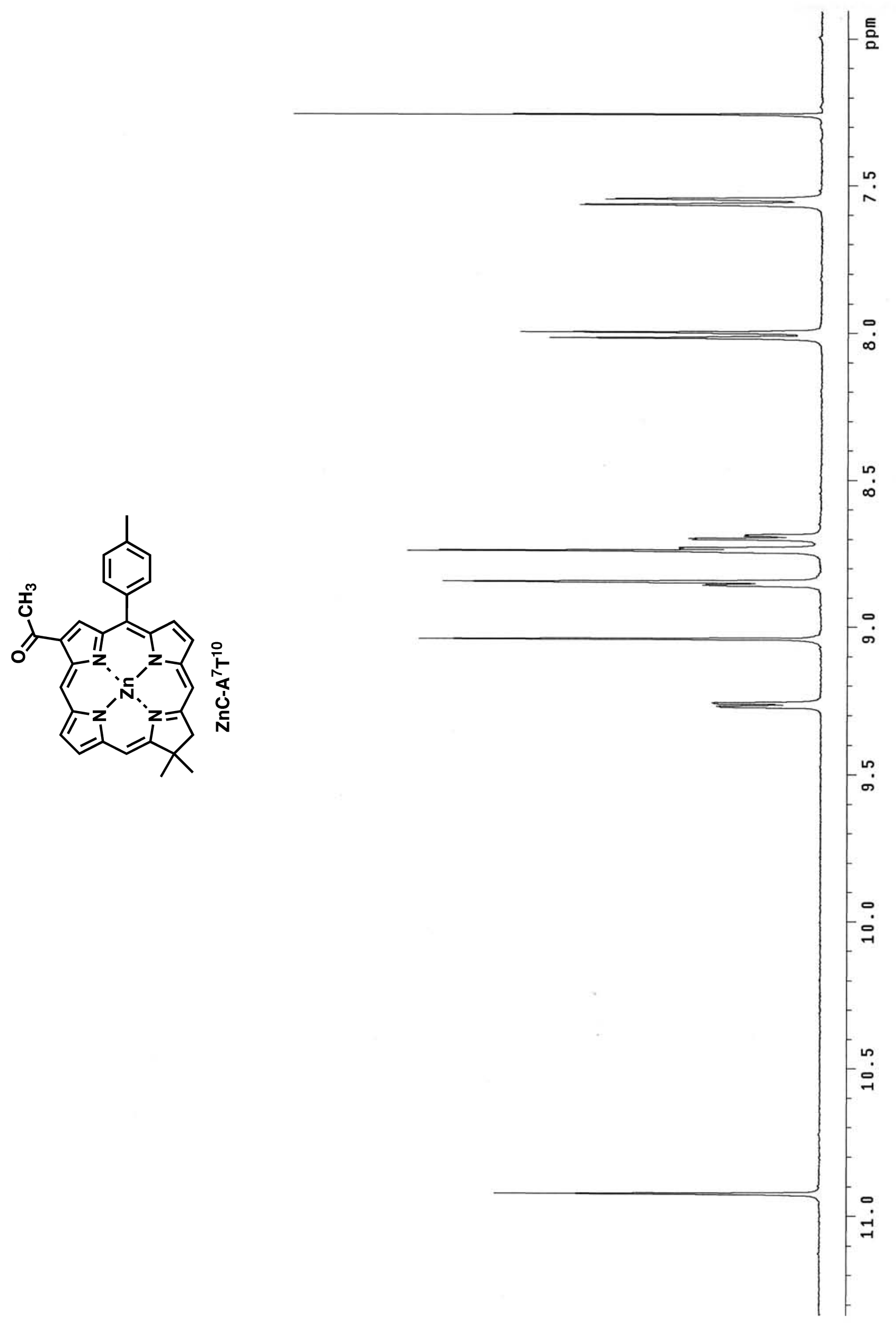




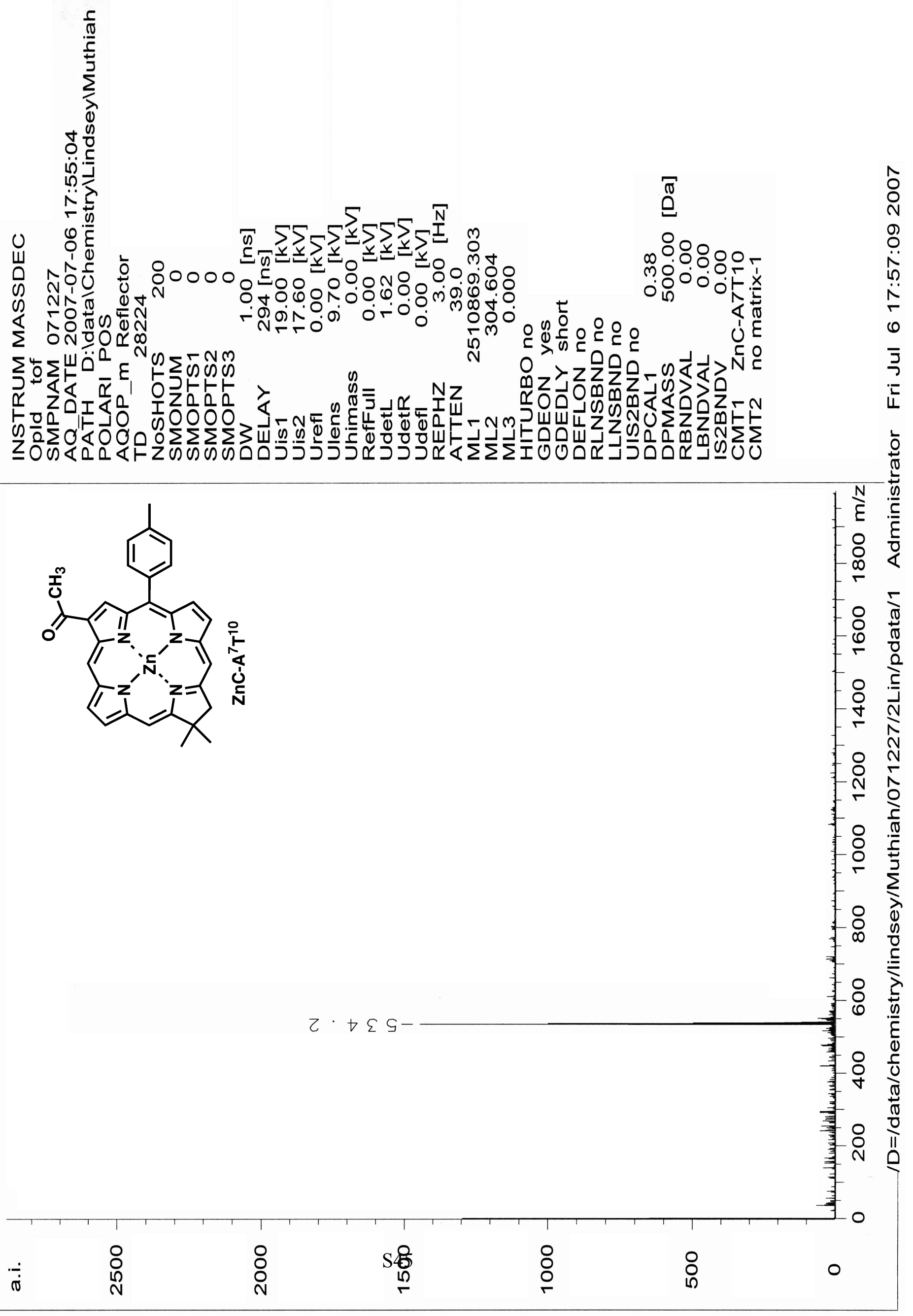



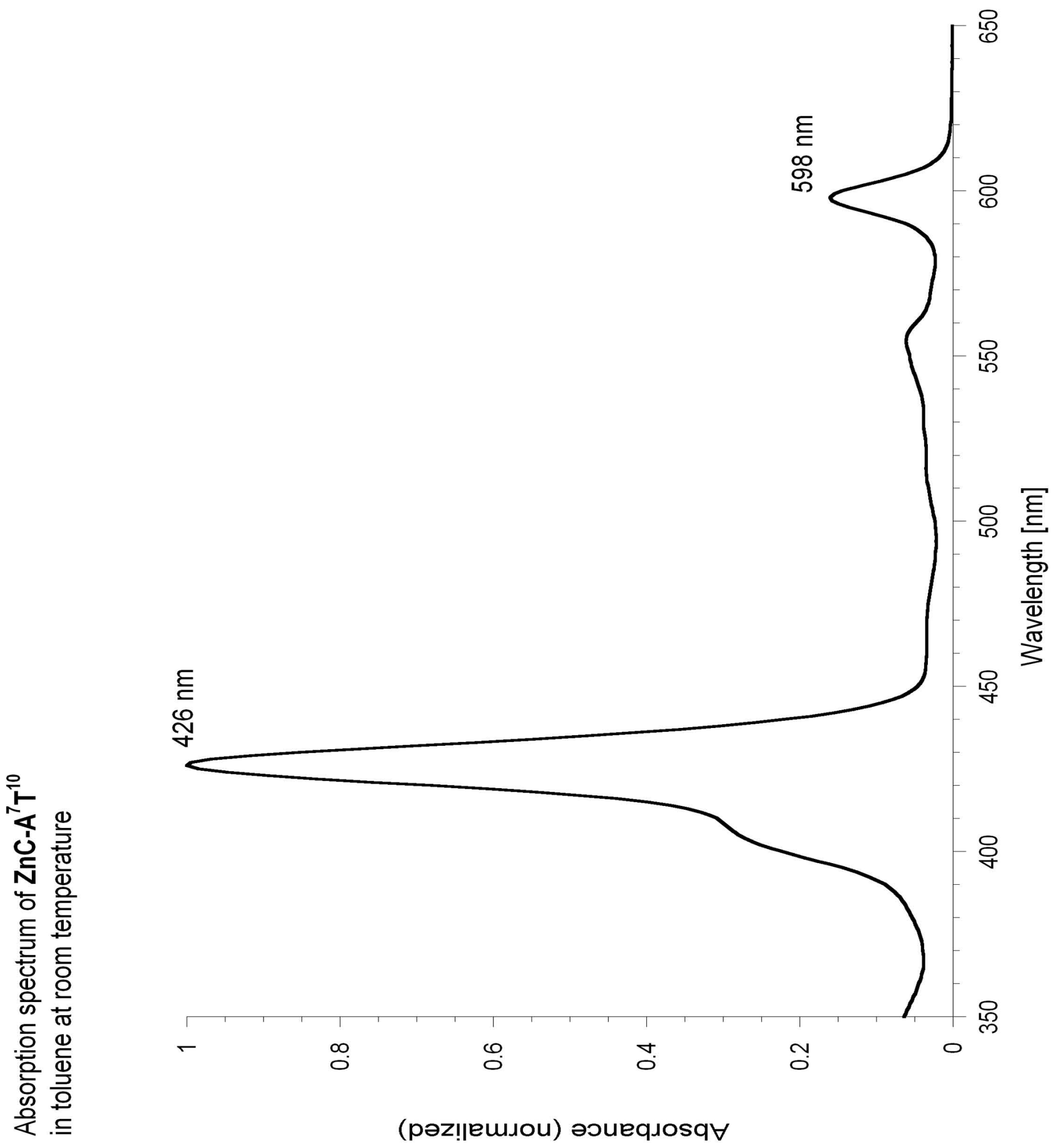


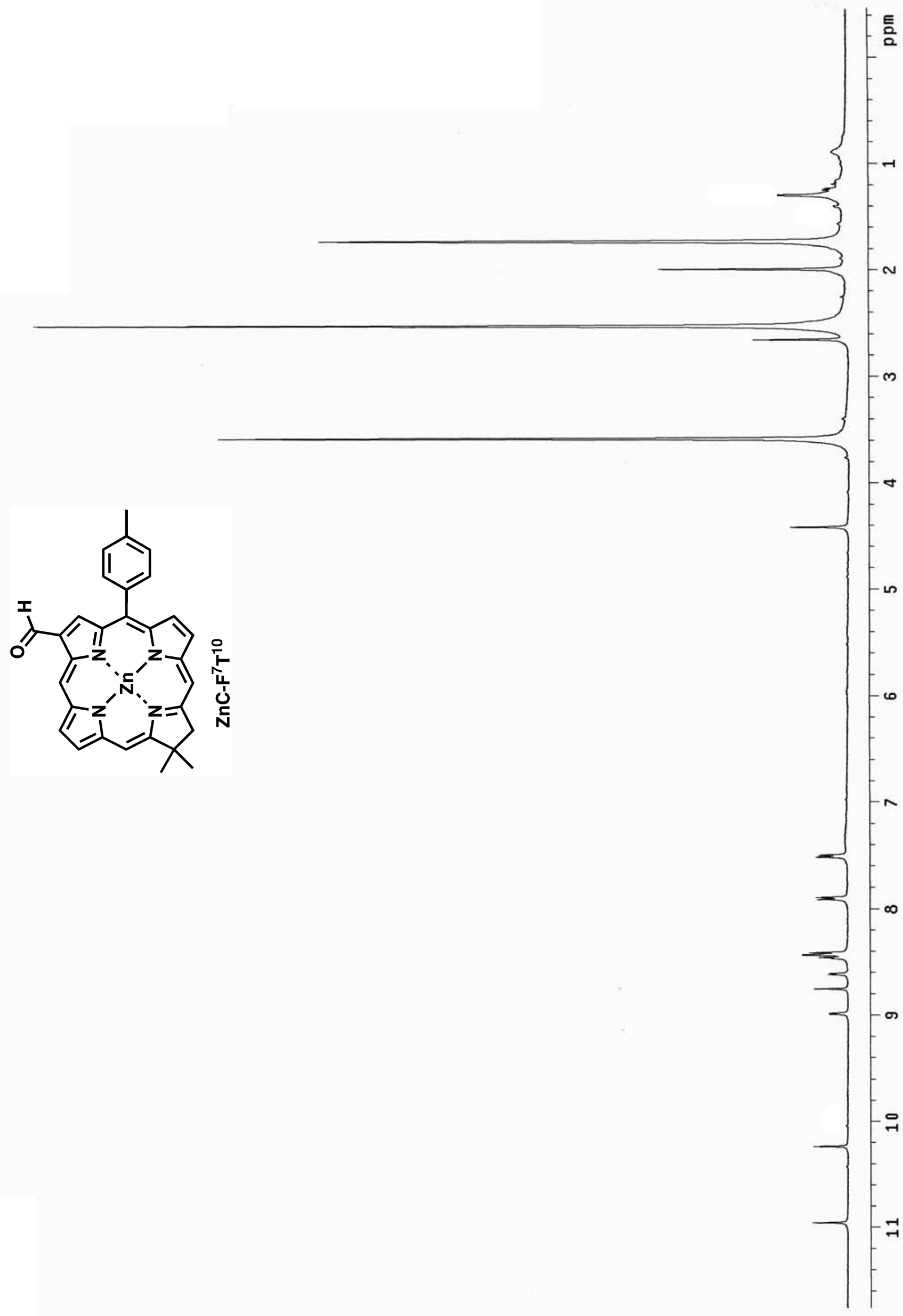




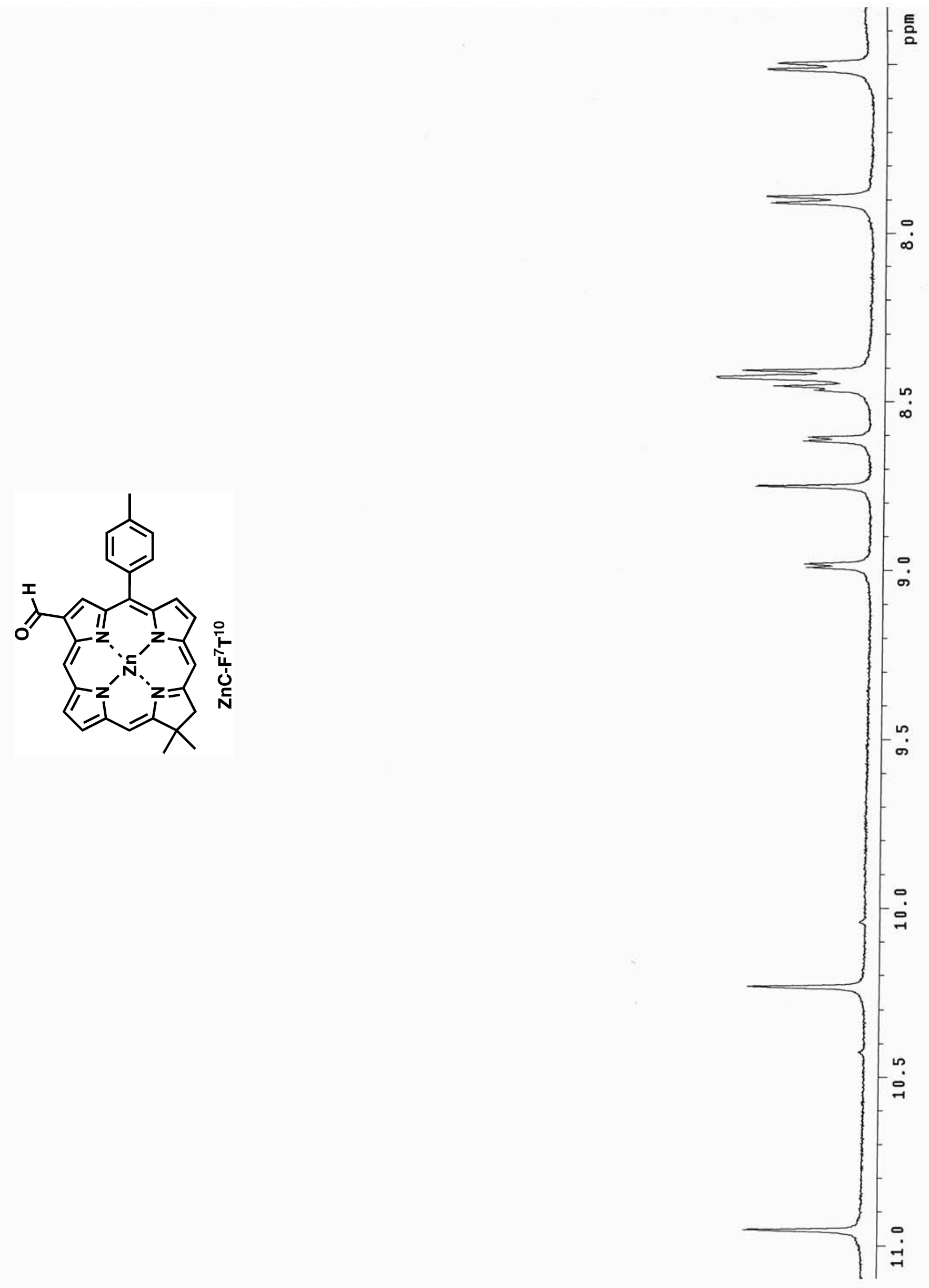




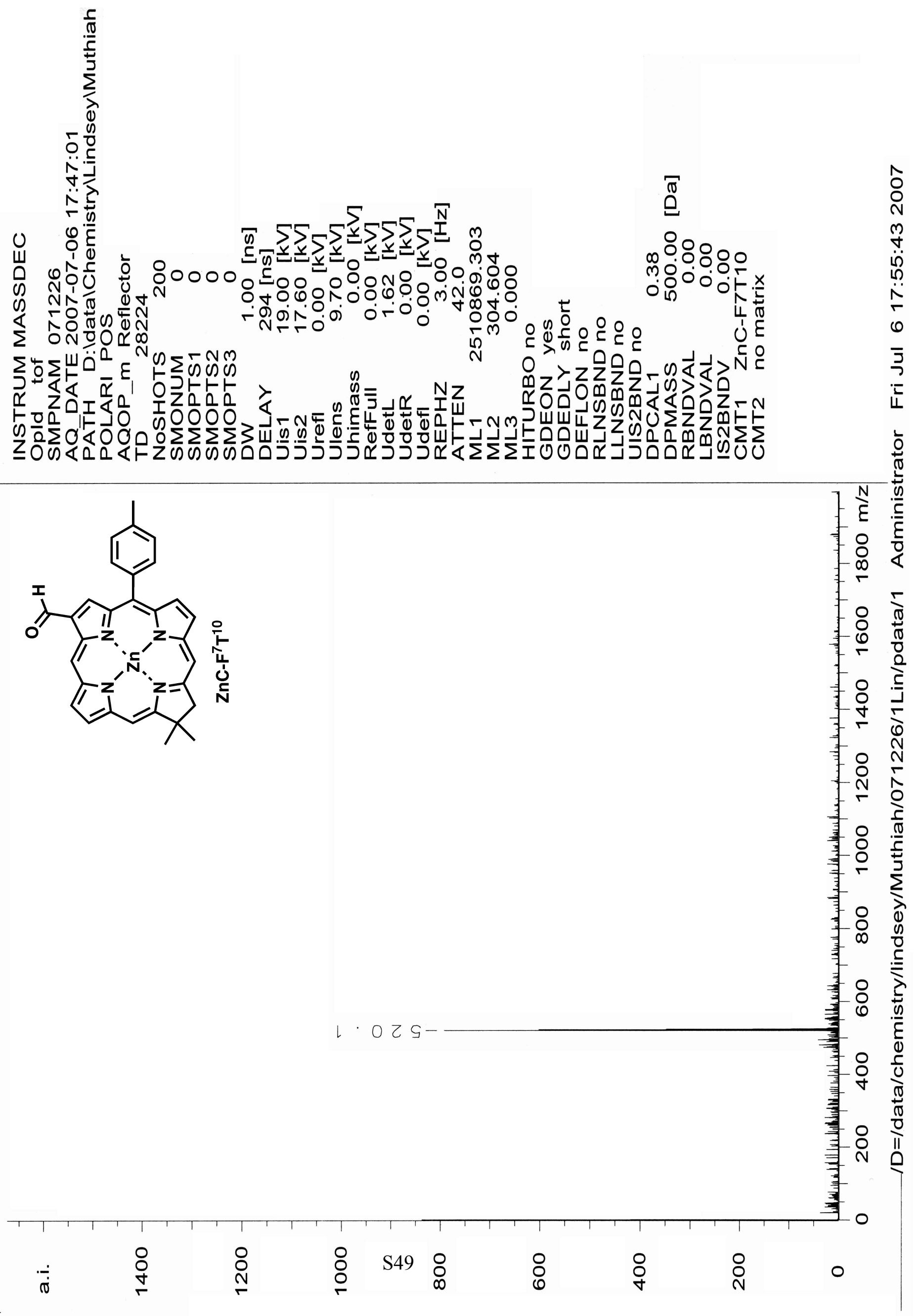



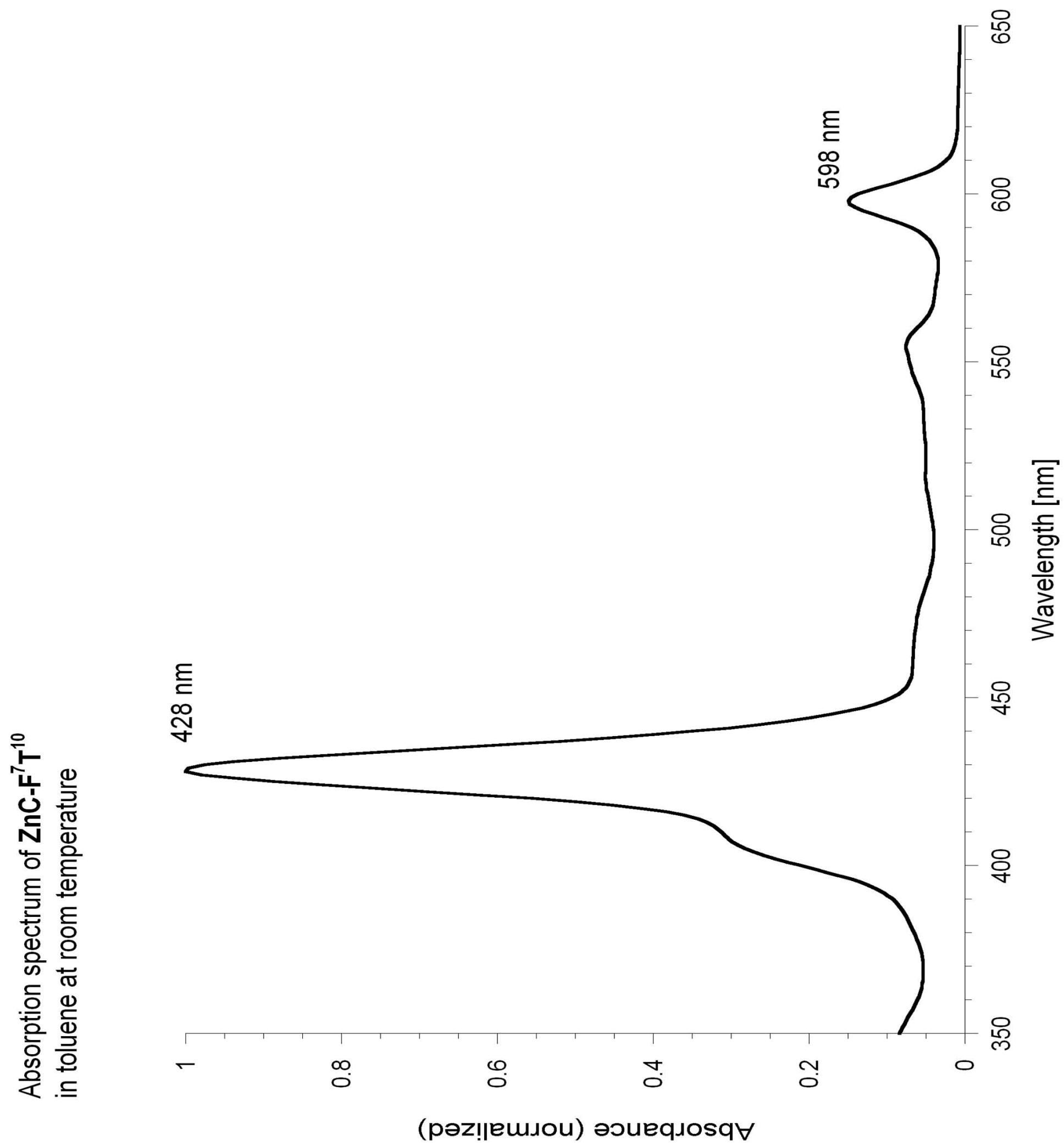


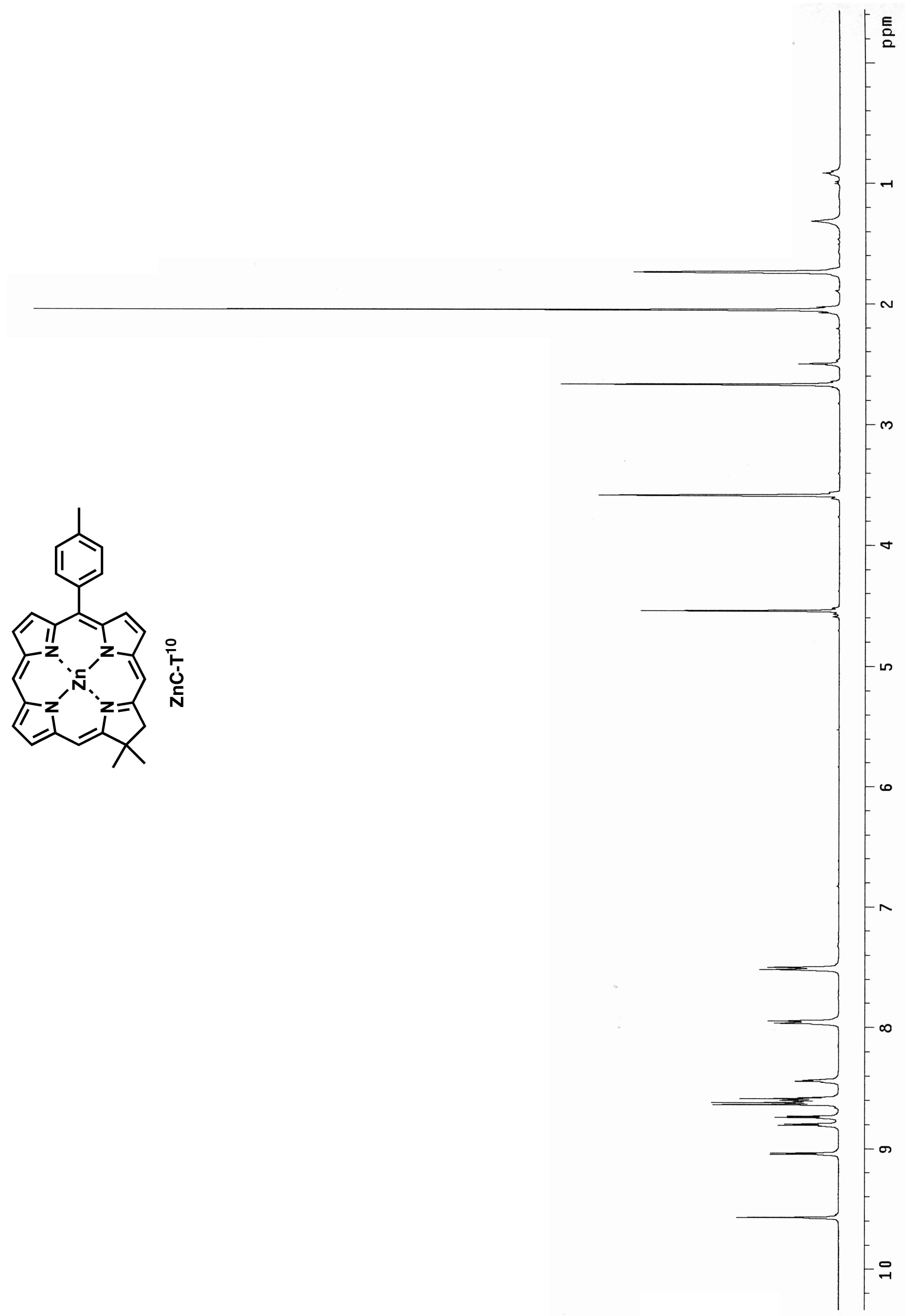




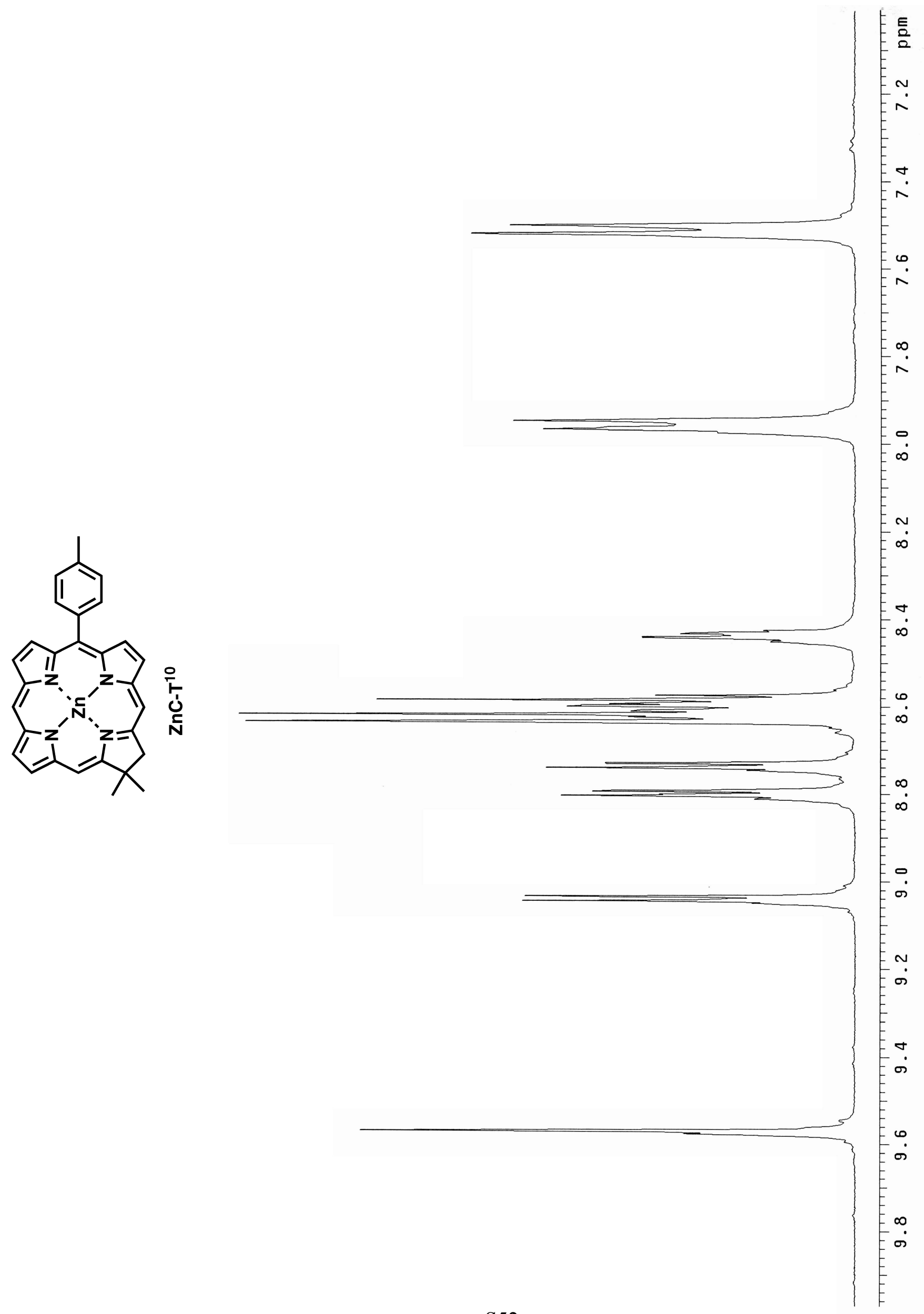




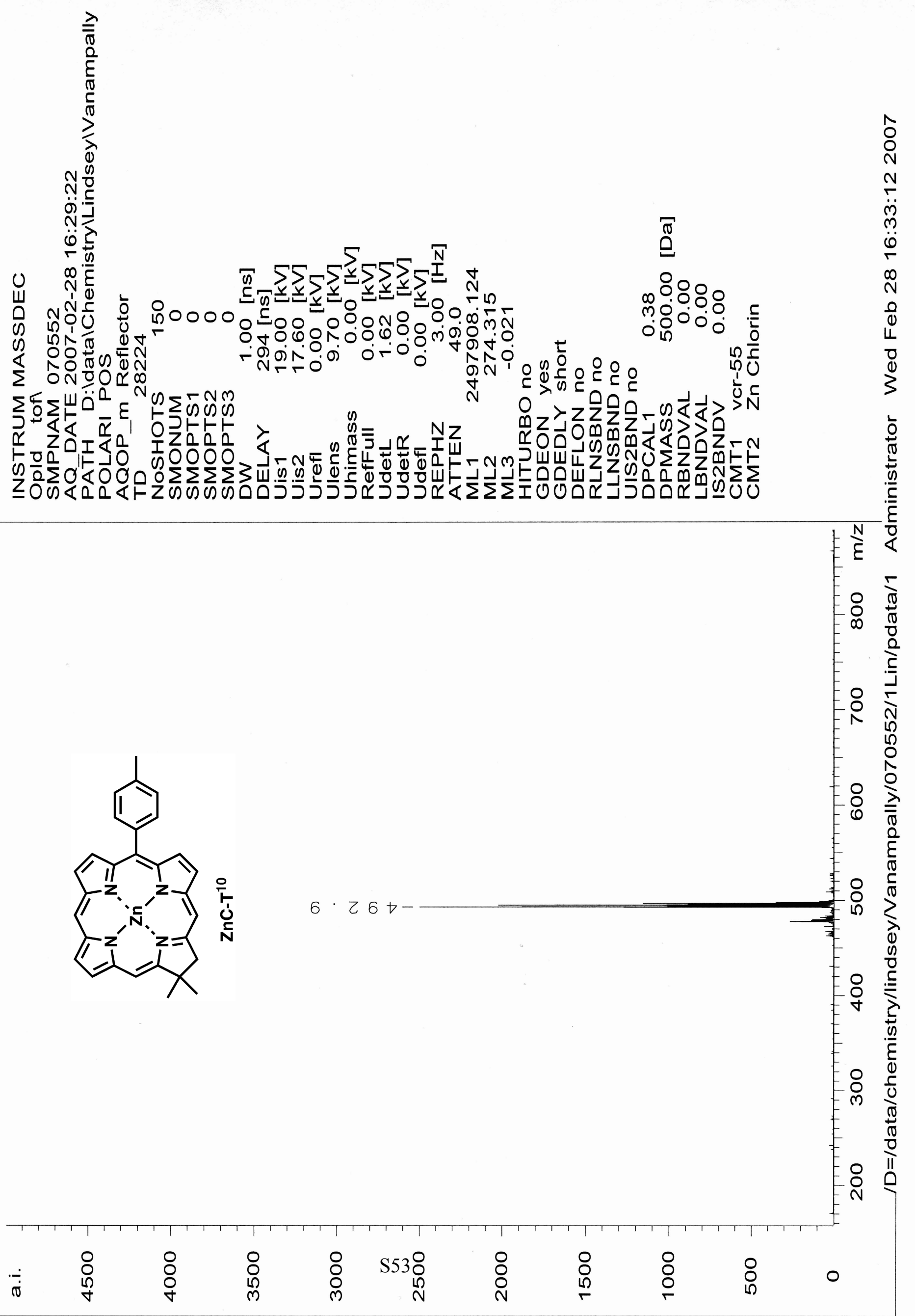




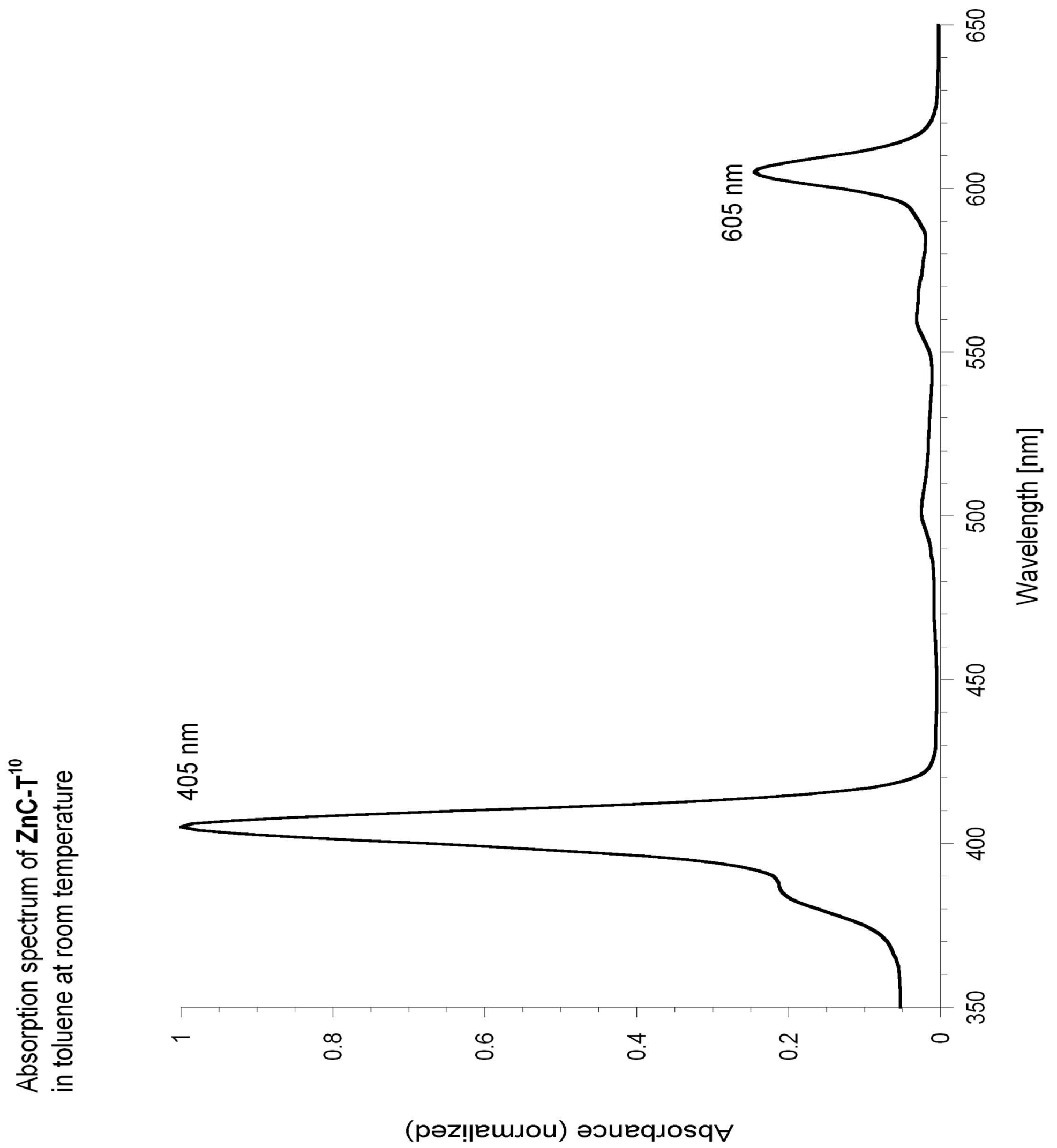




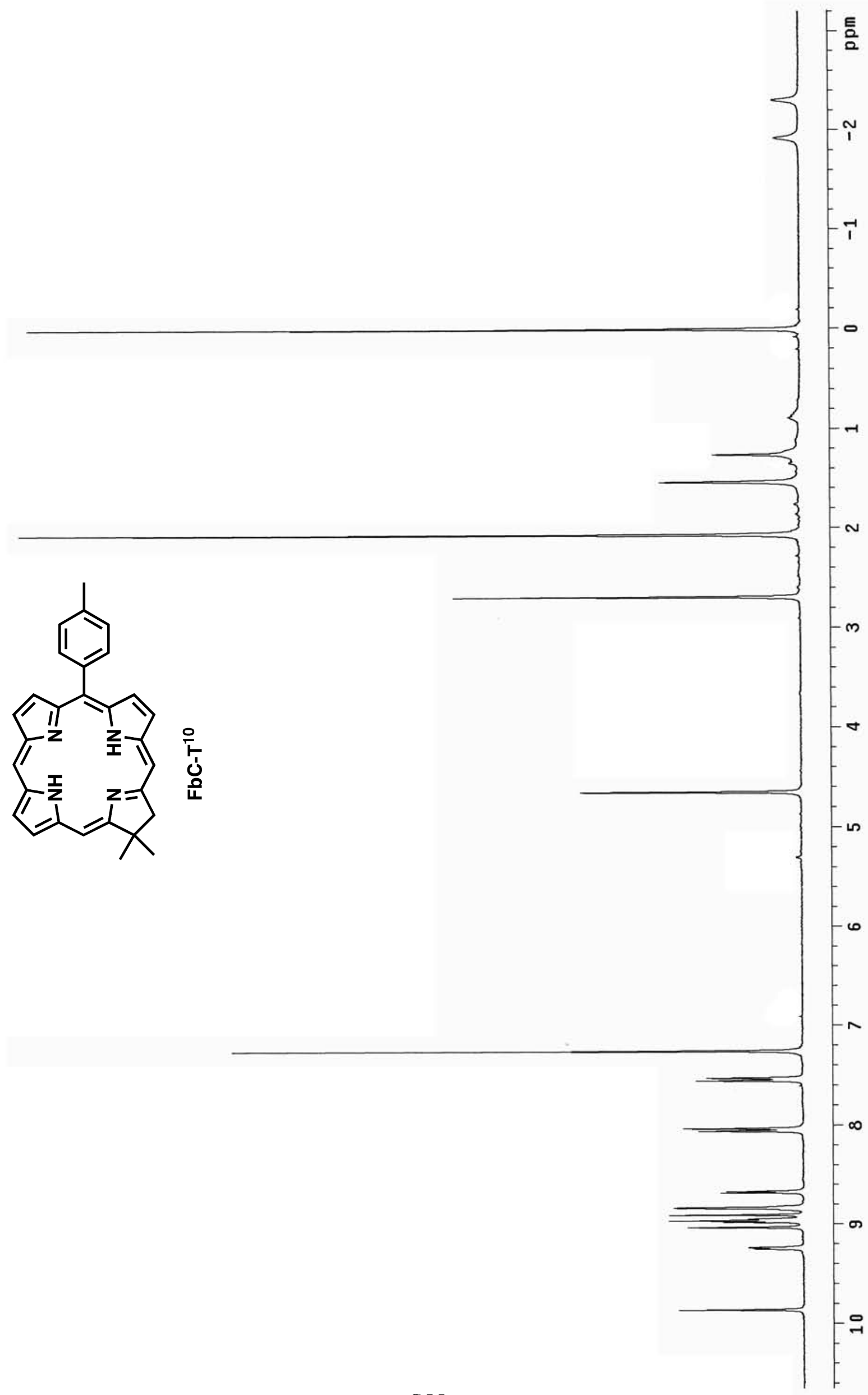




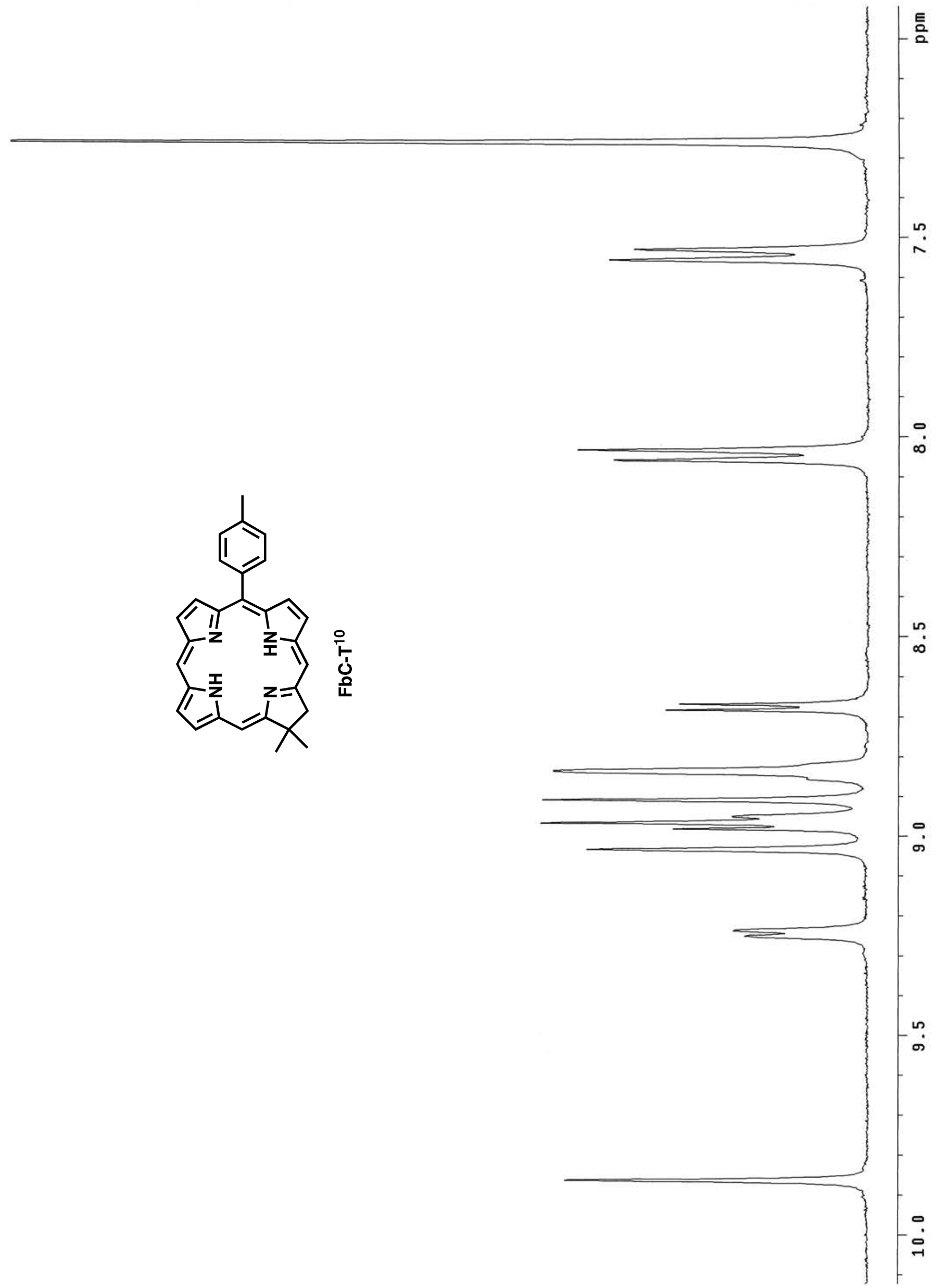




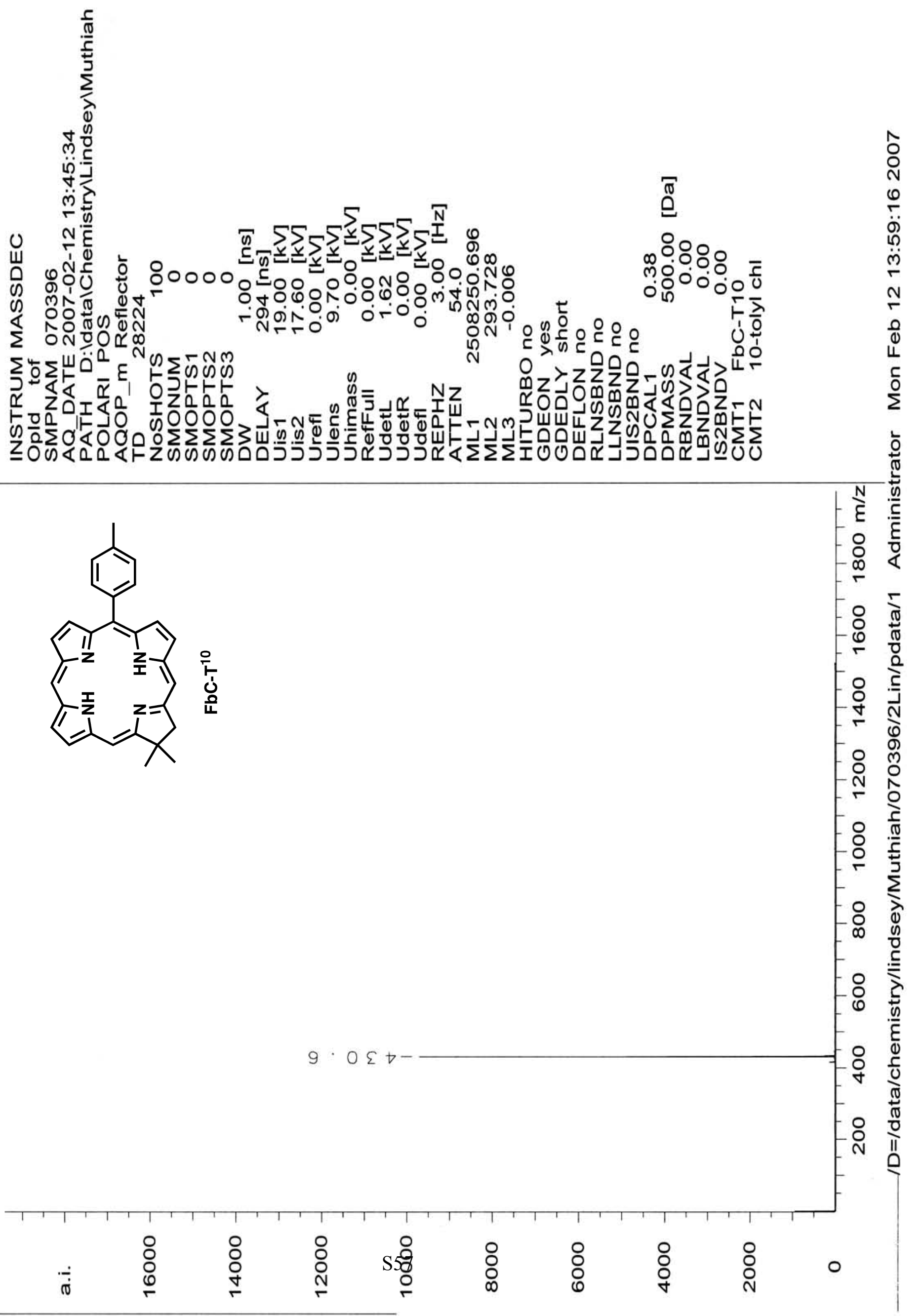



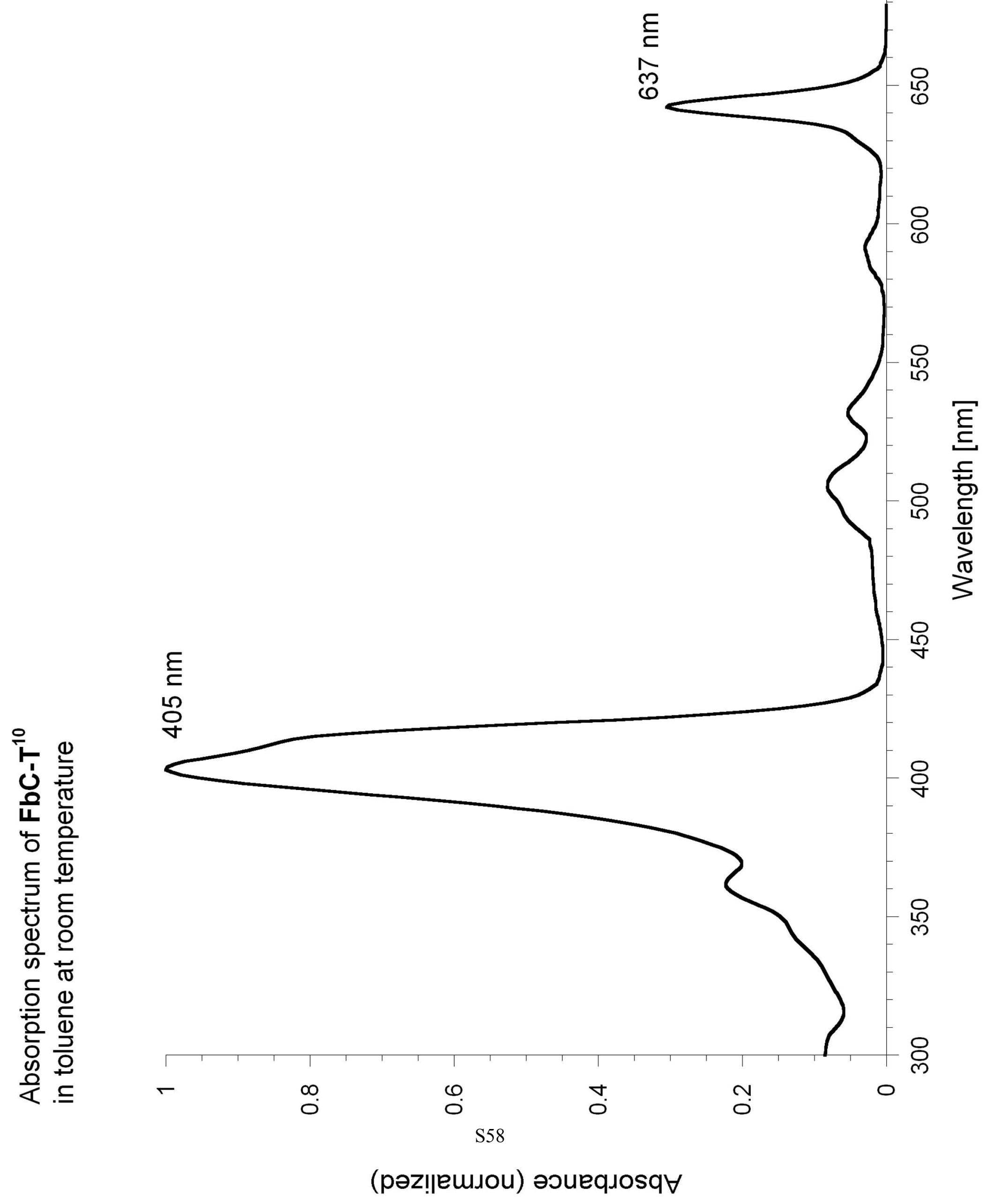


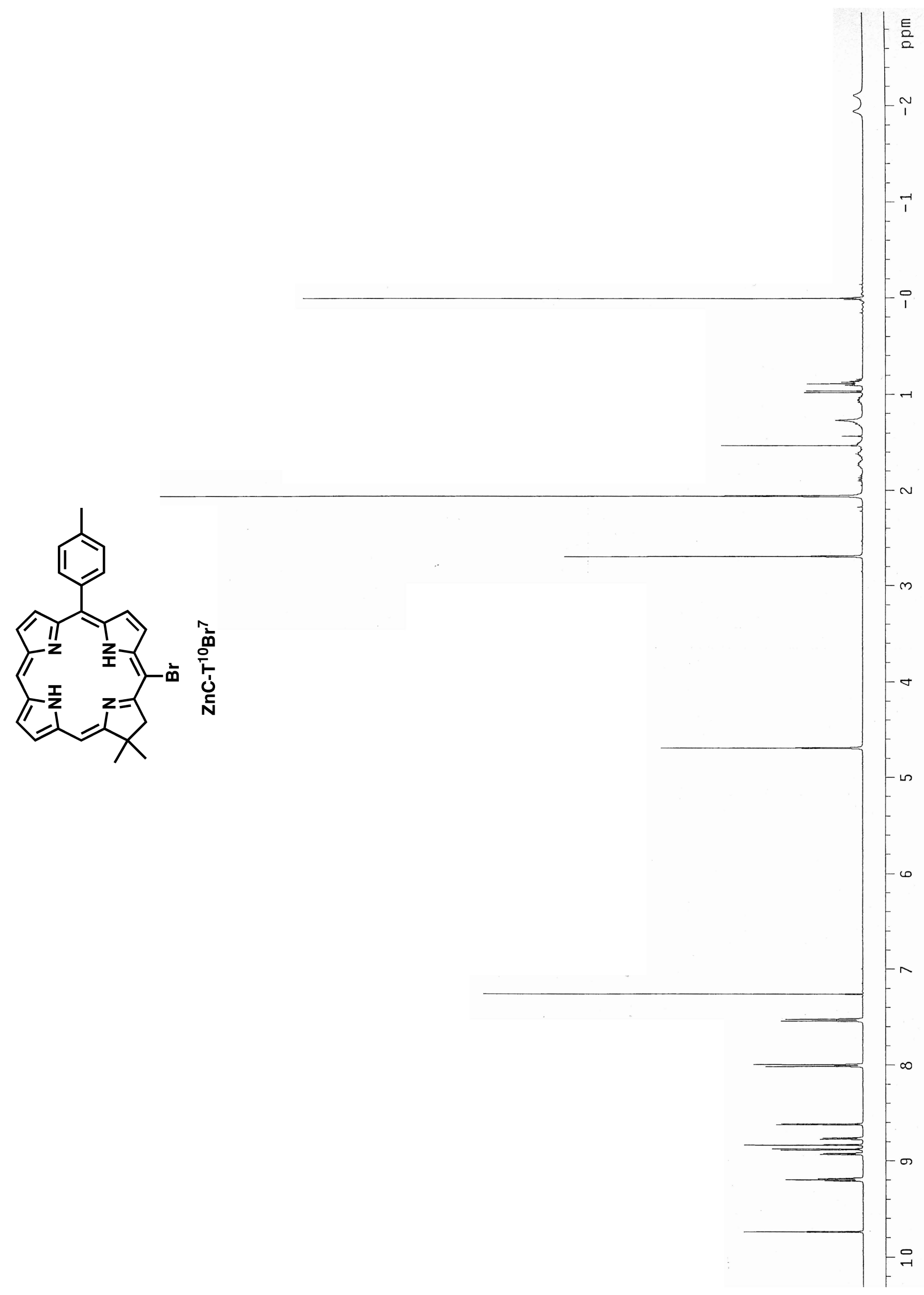




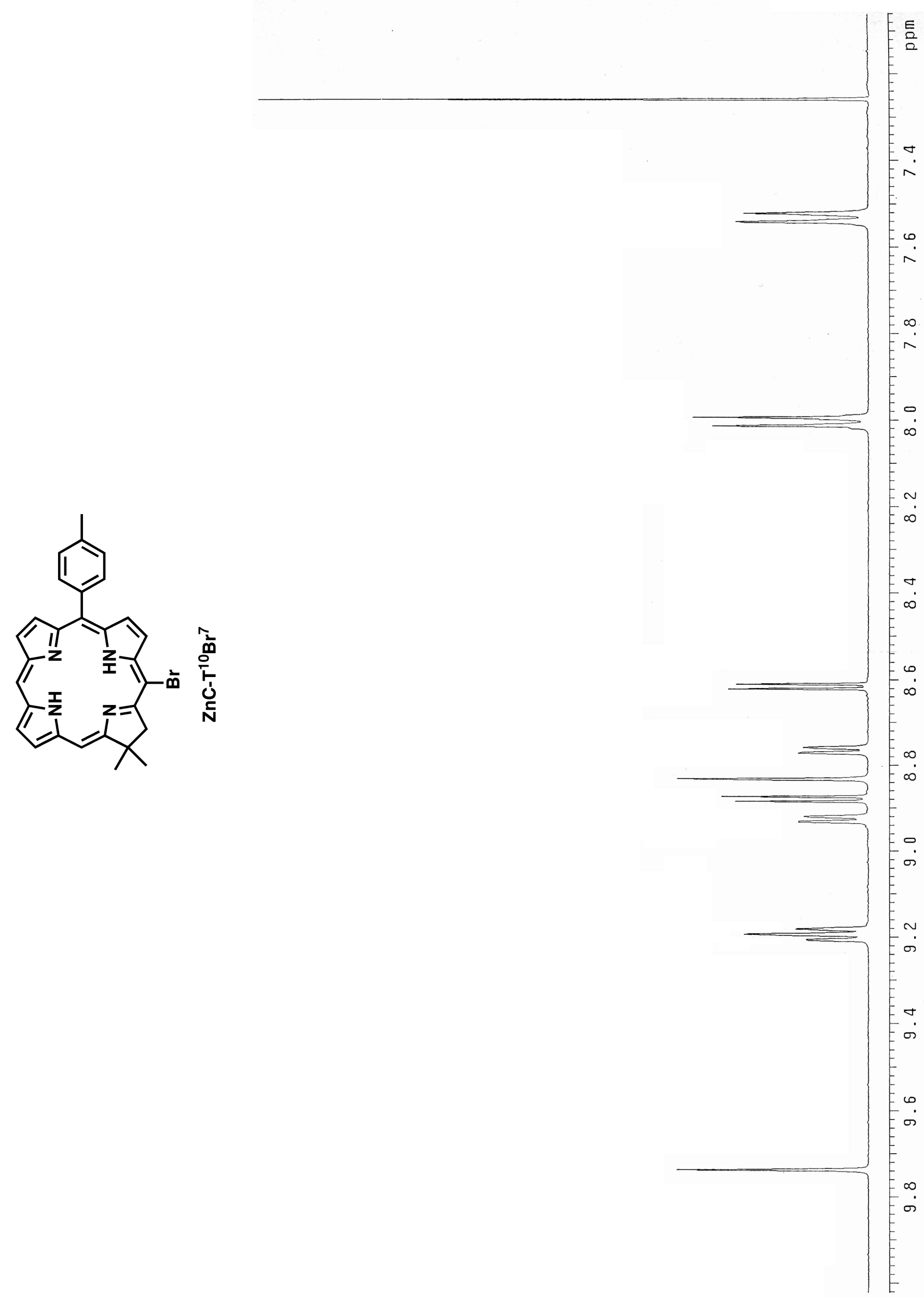




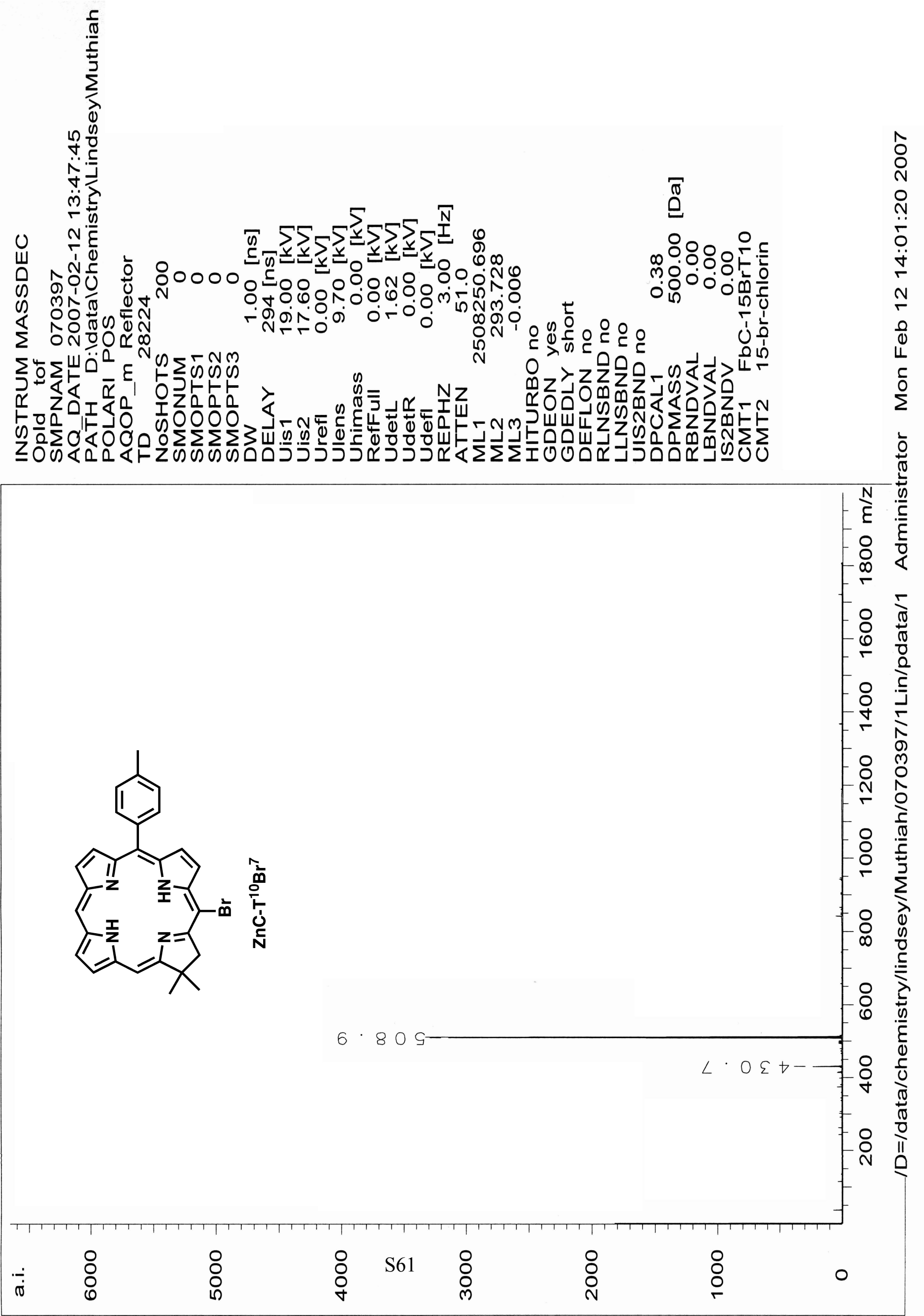




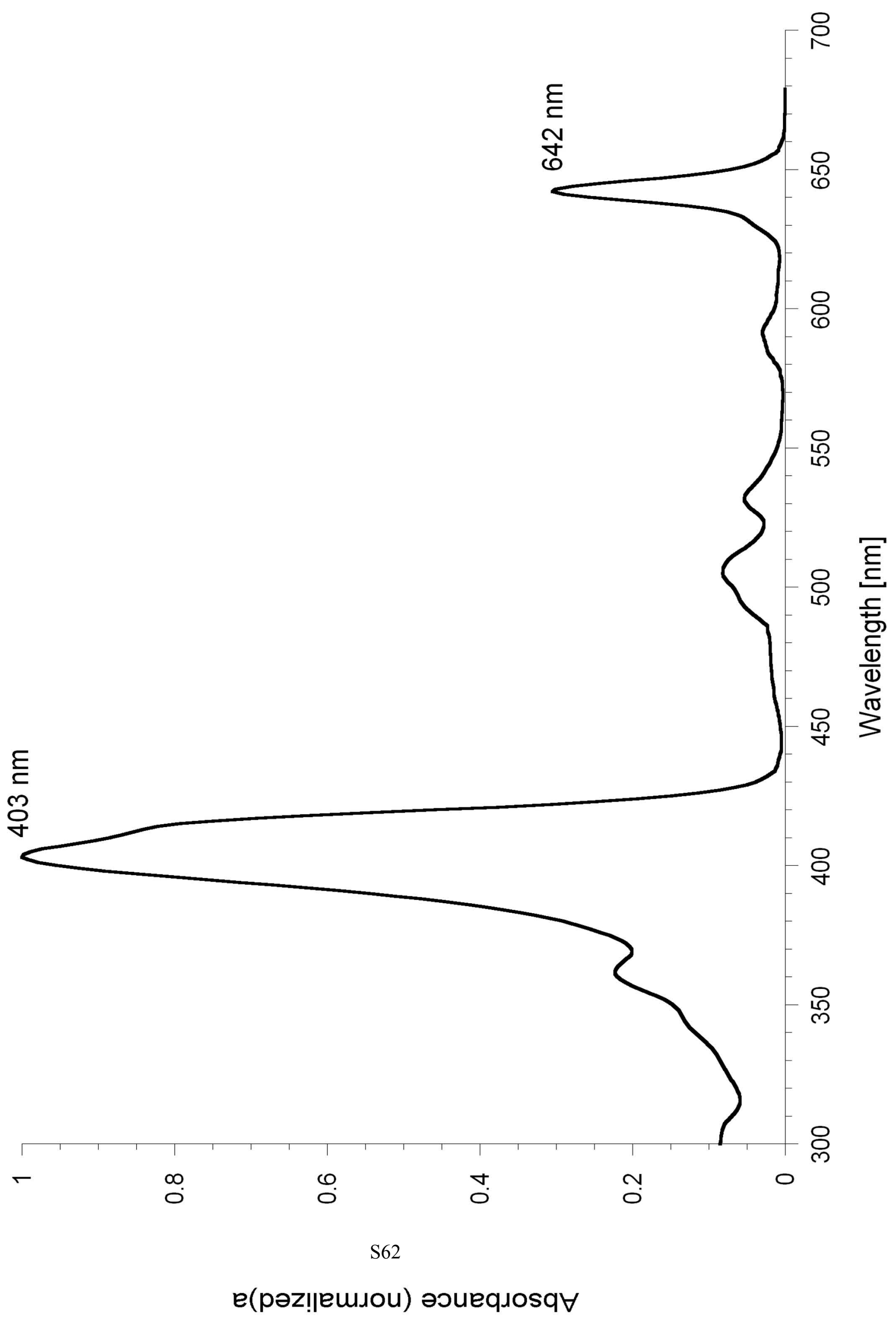




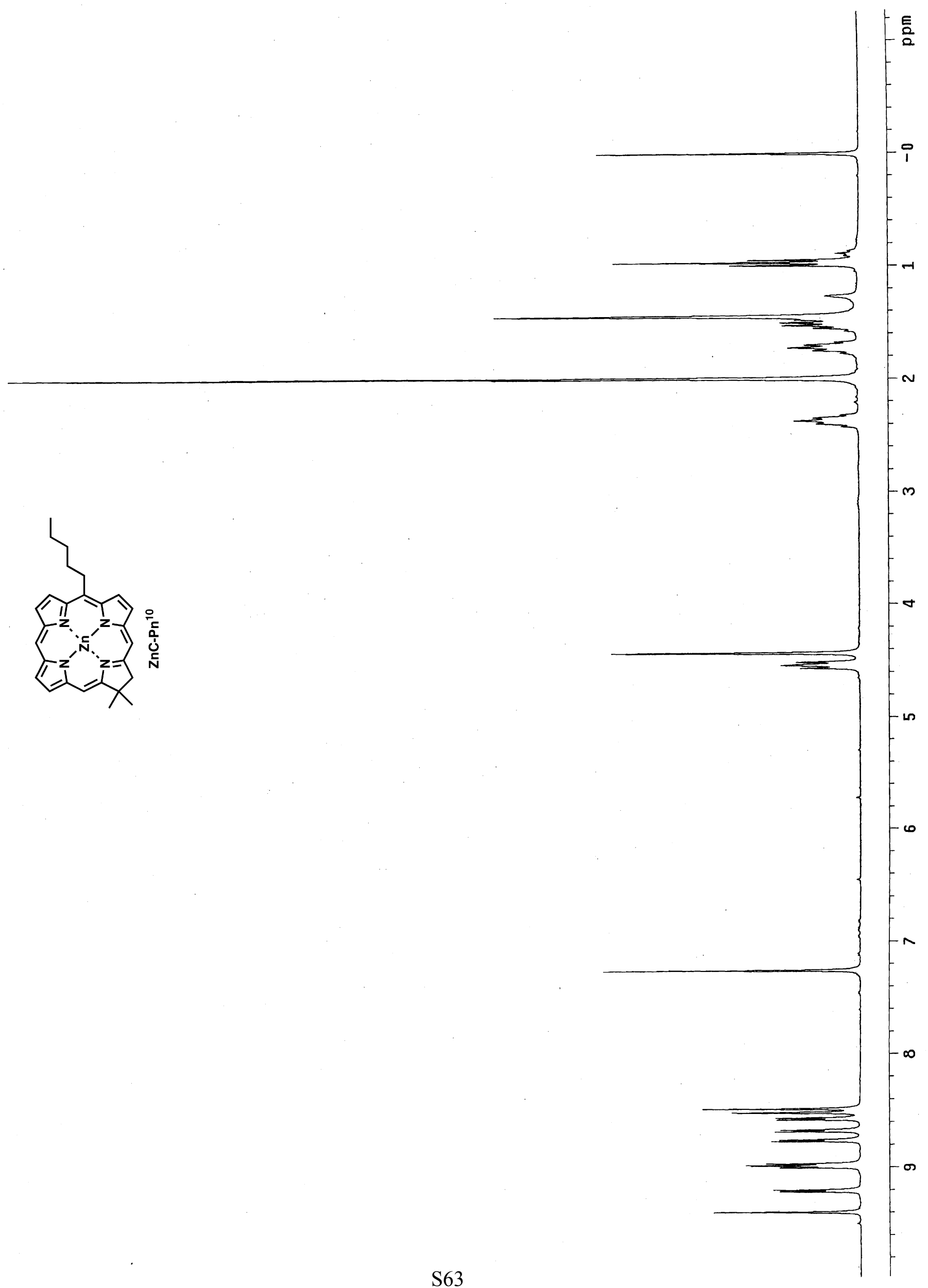




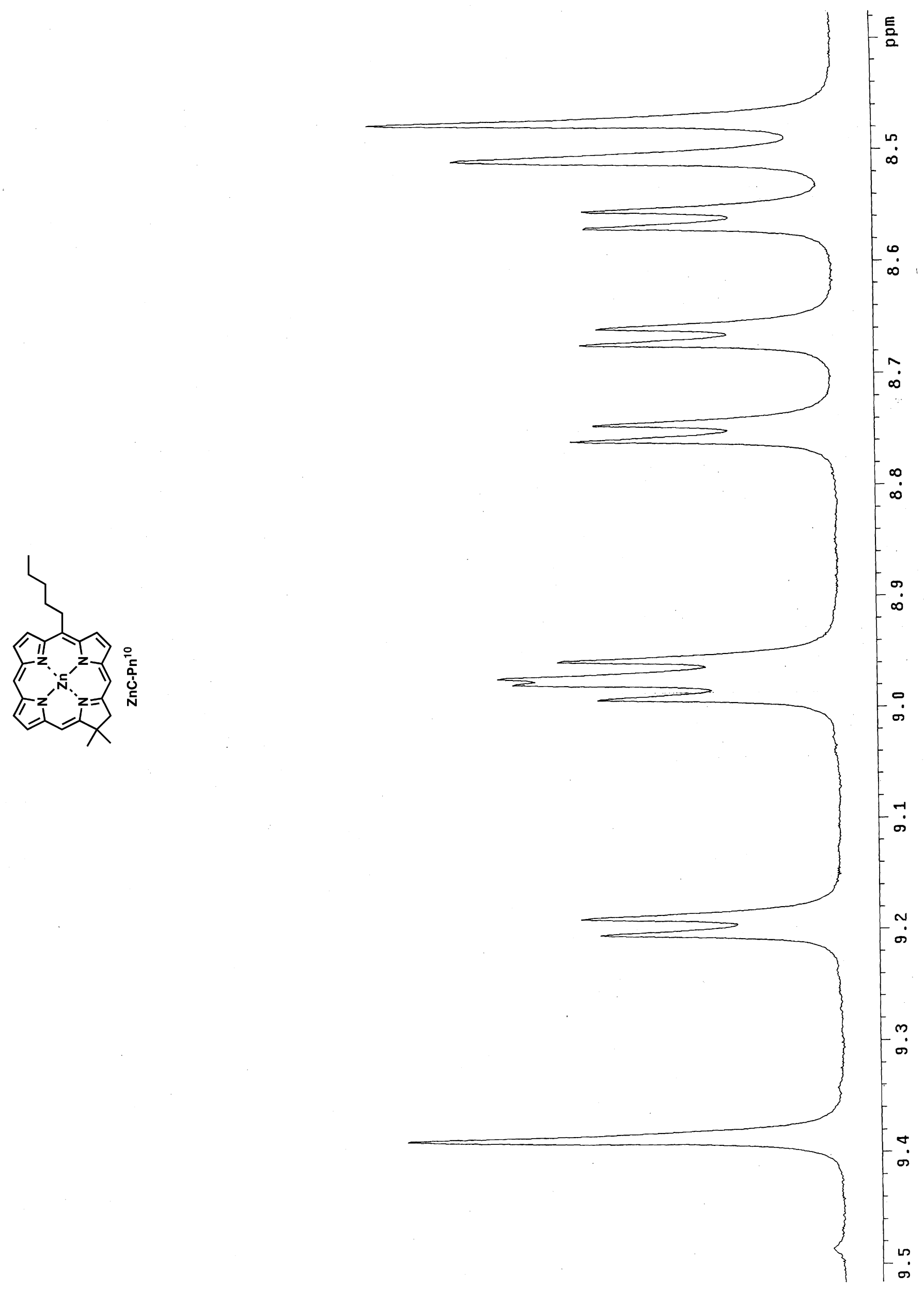




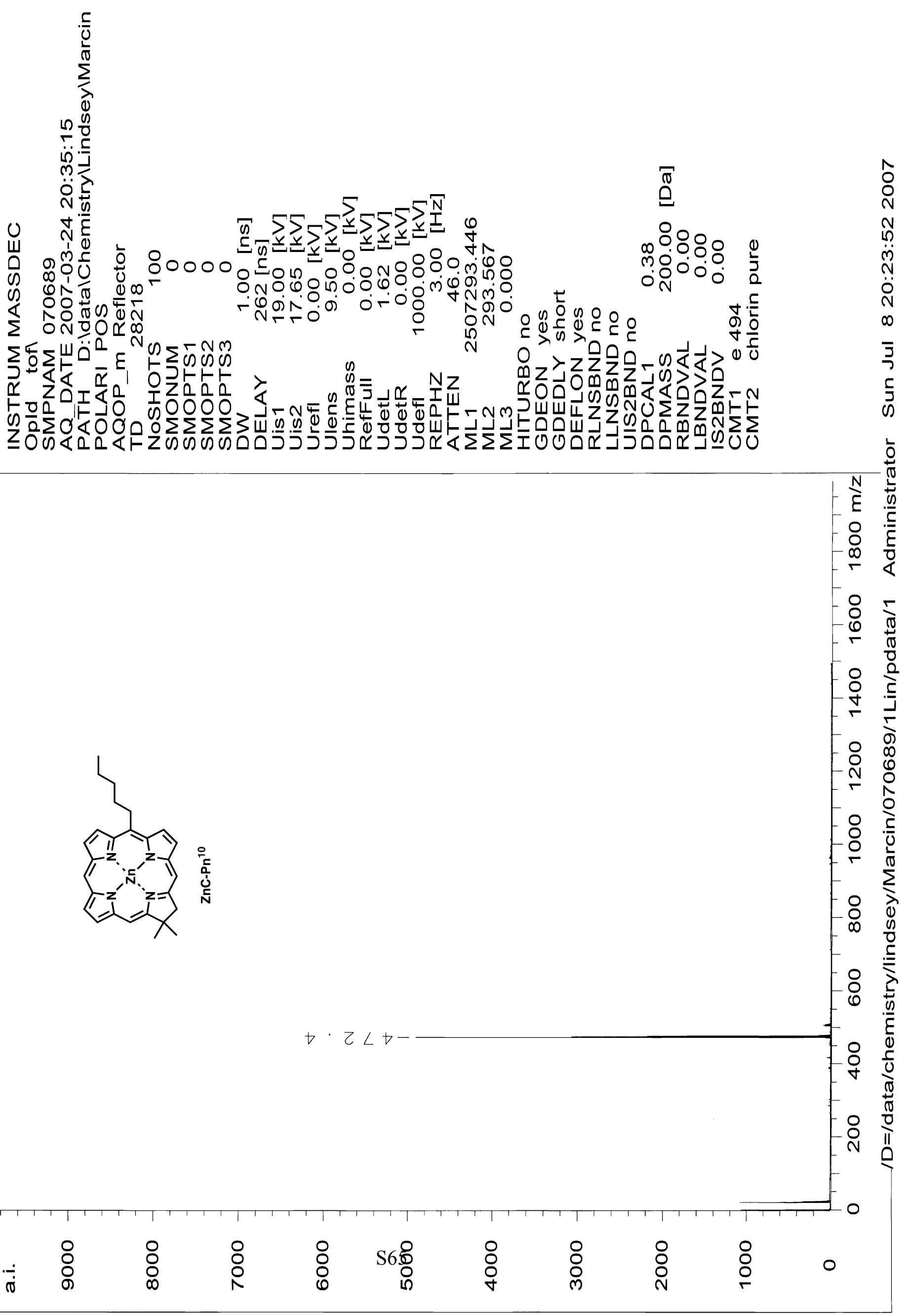




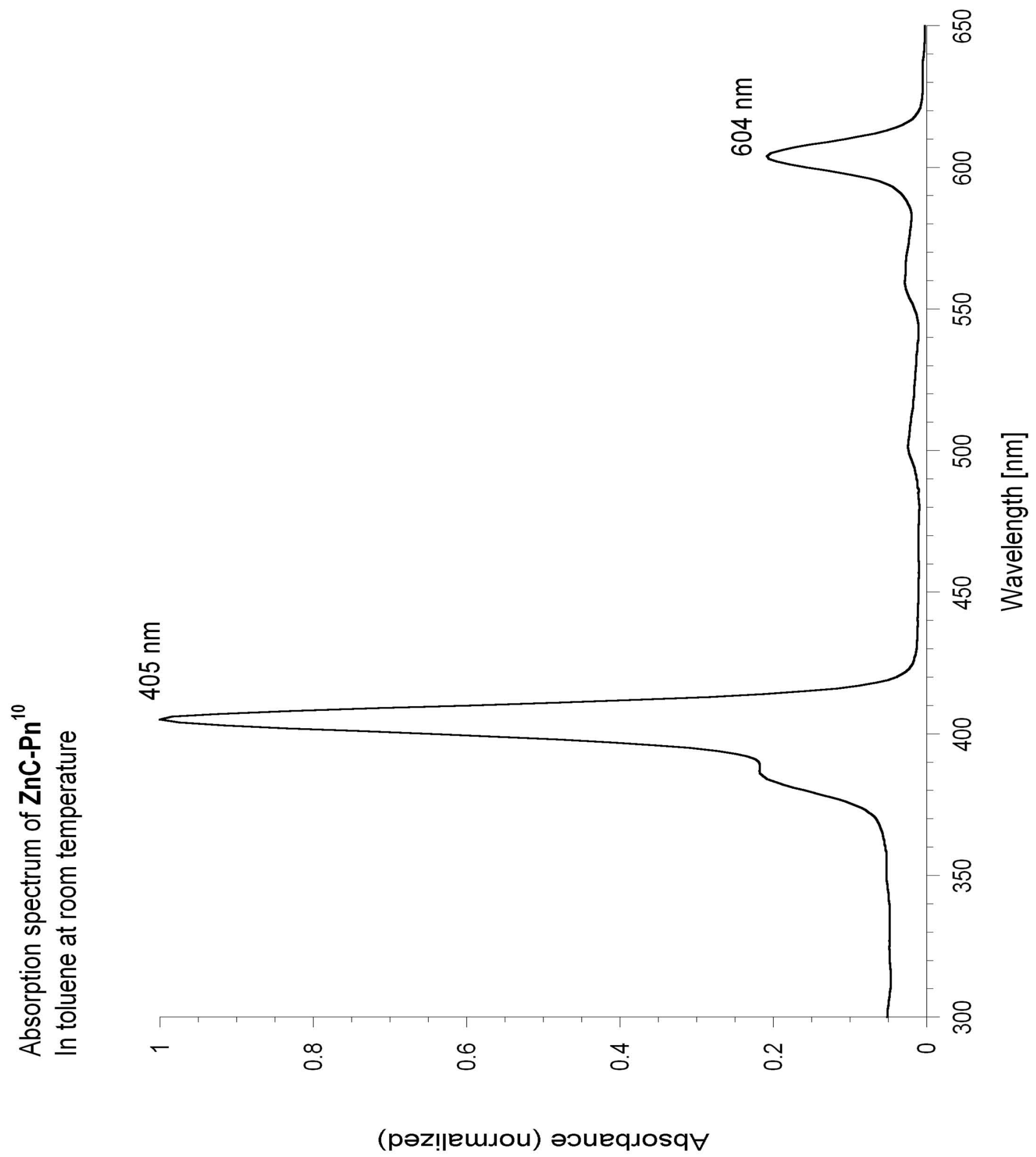




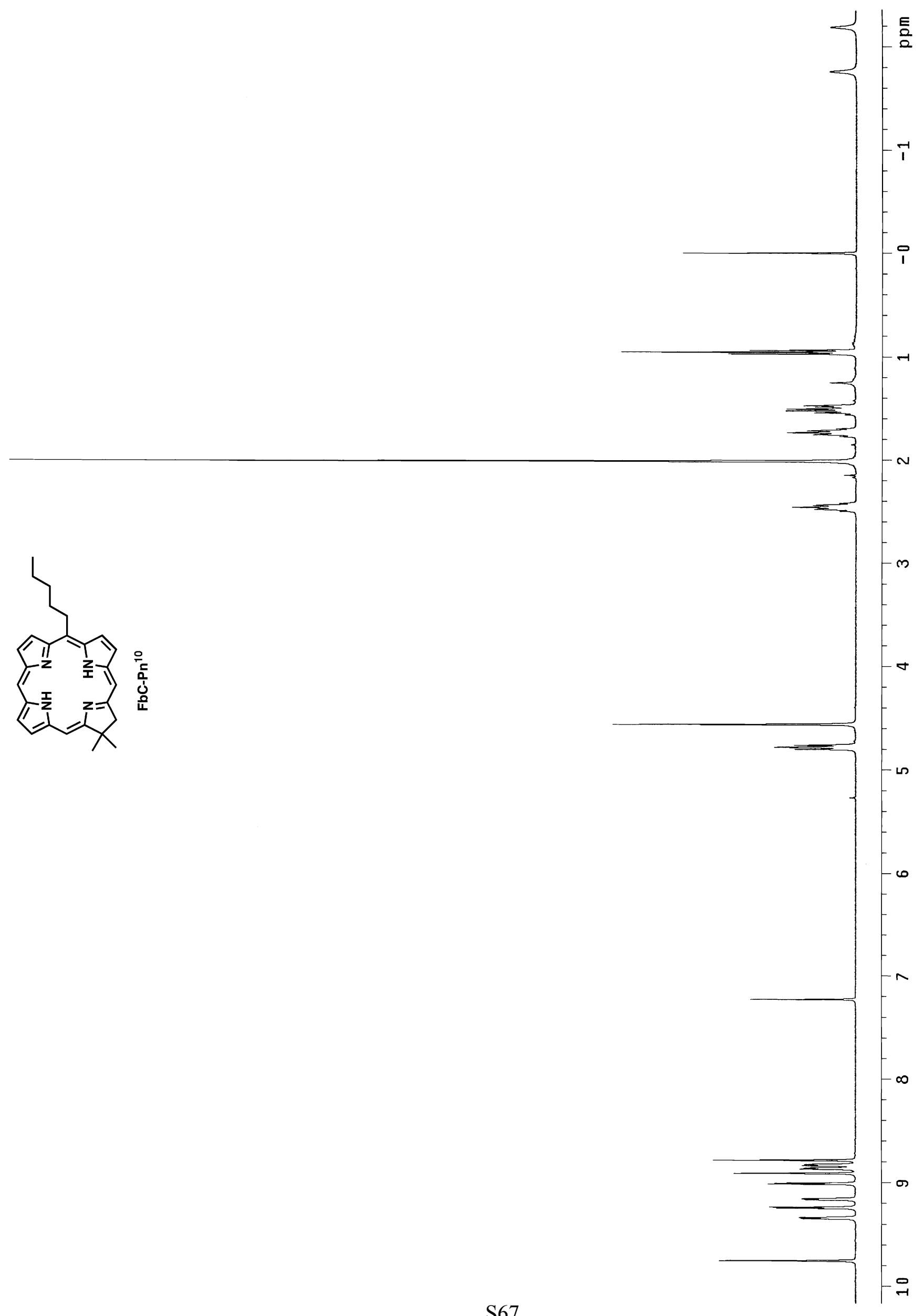




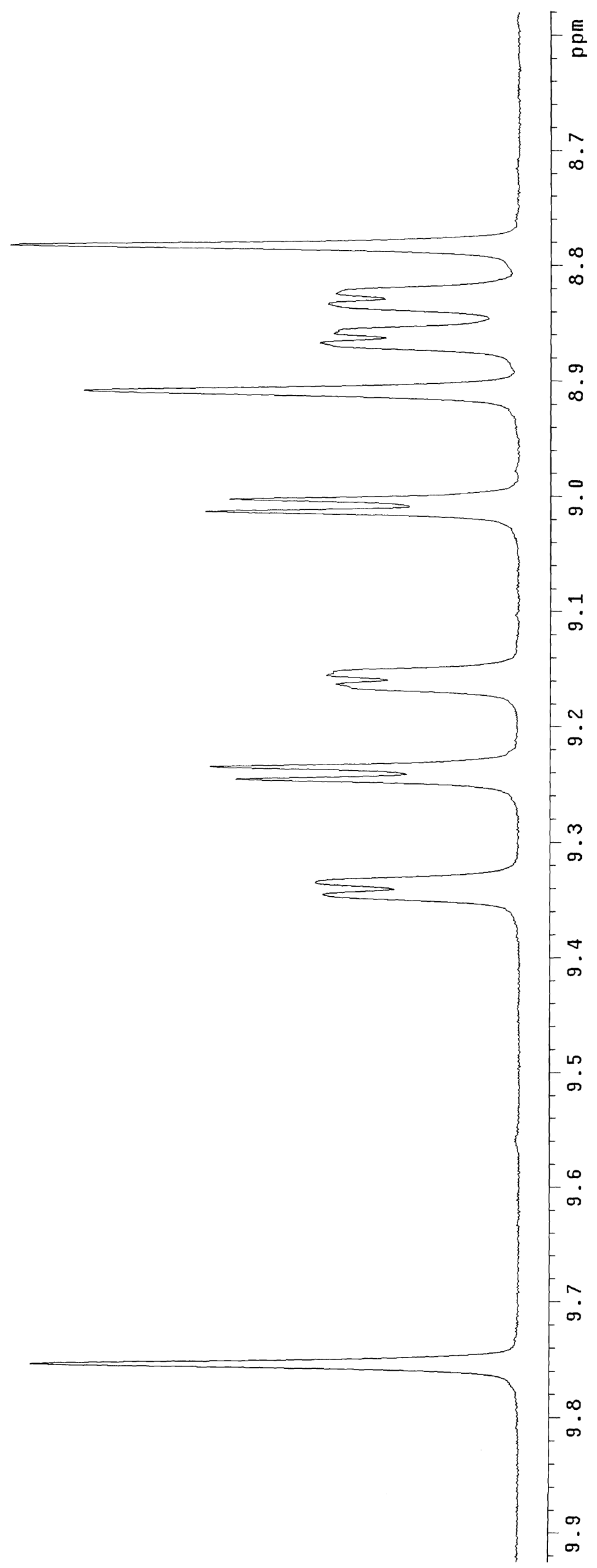

S68 


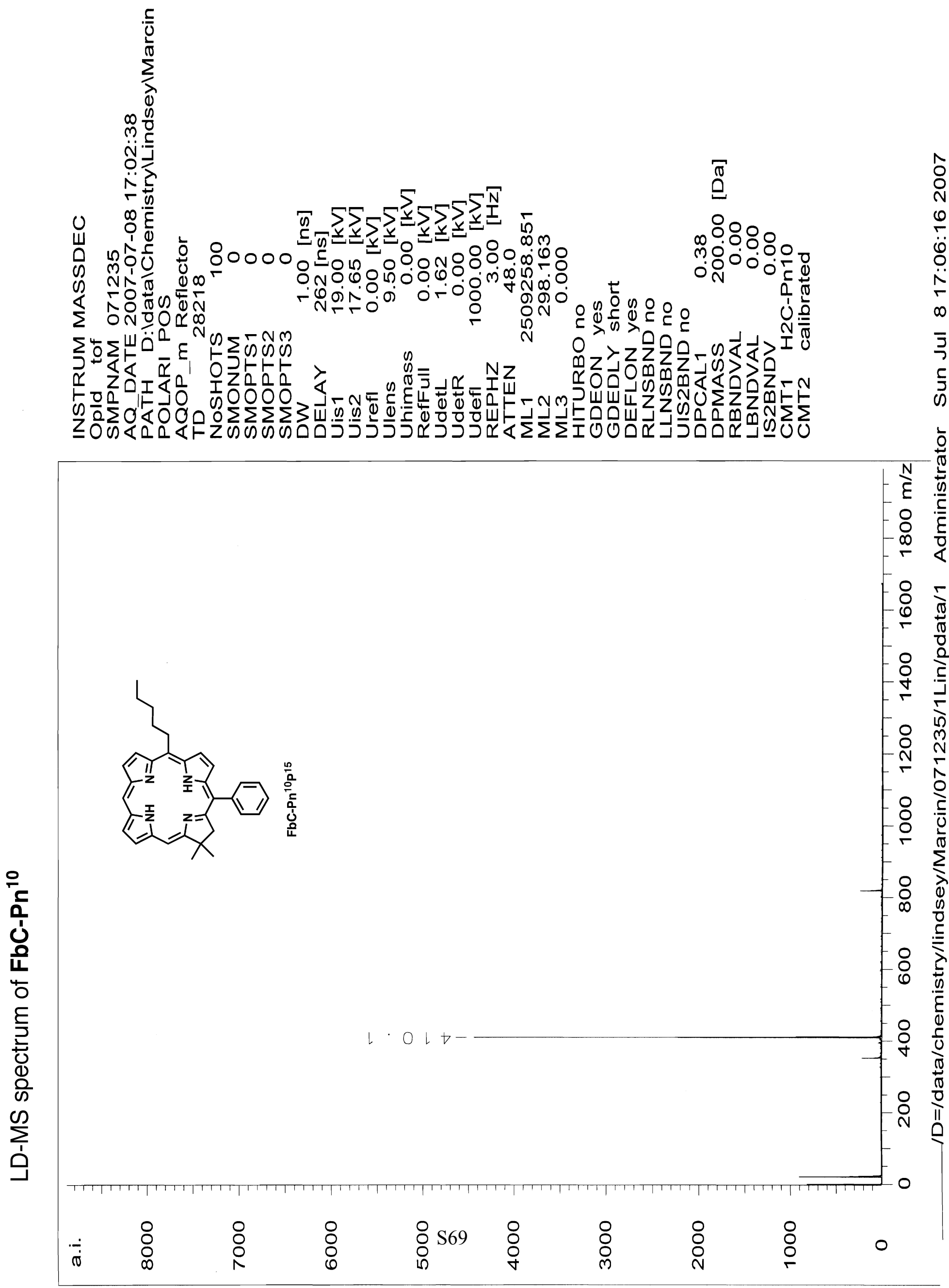



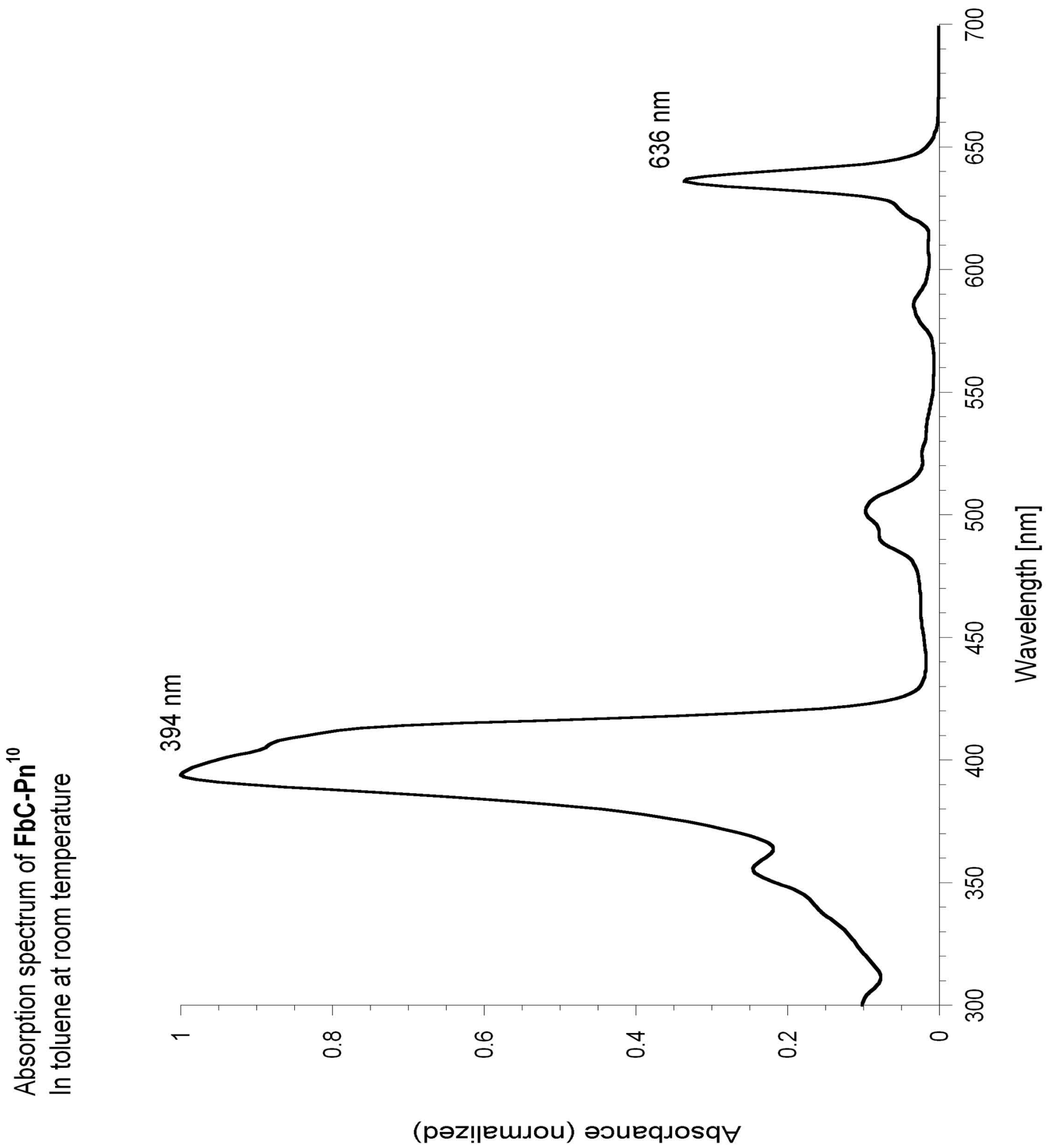


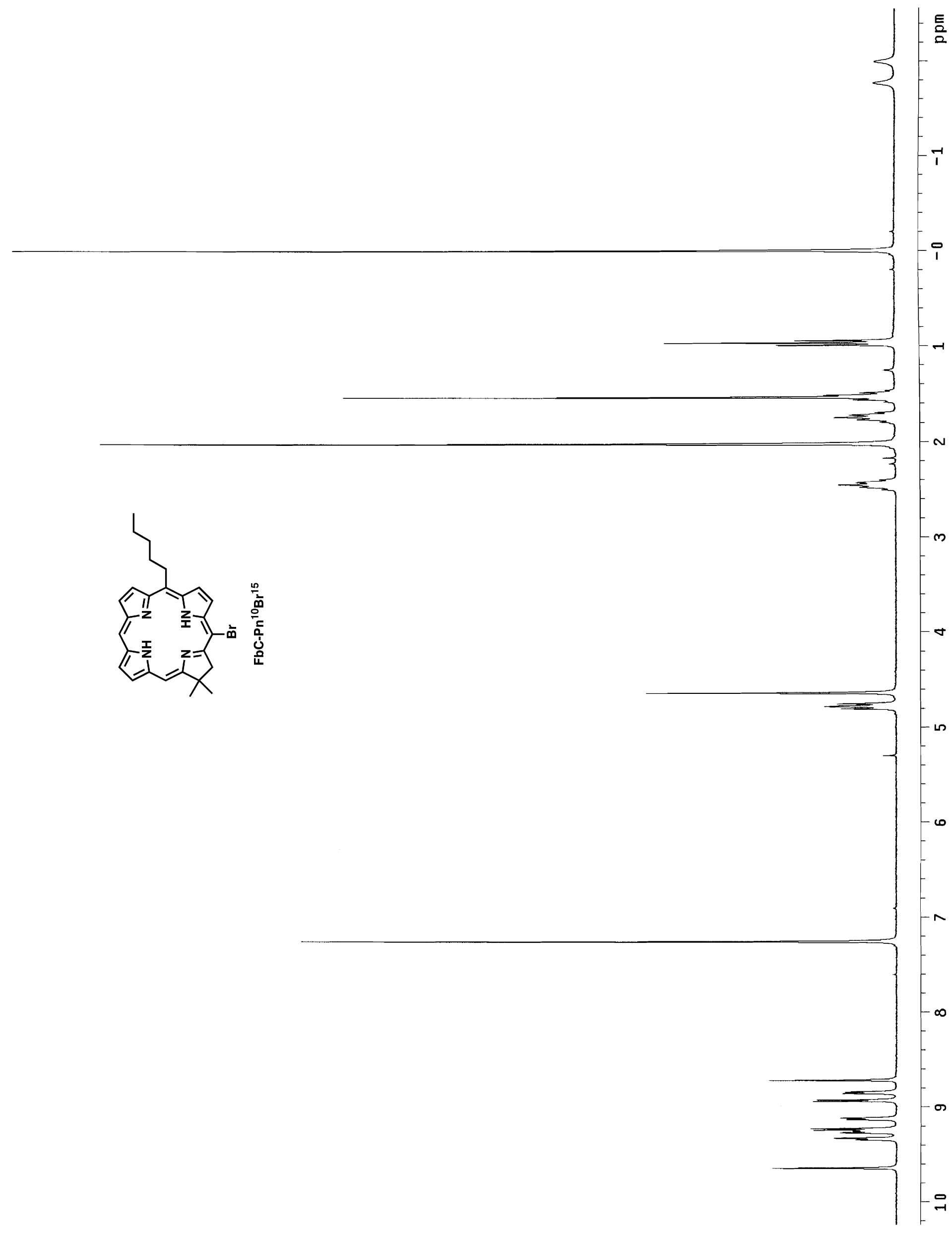




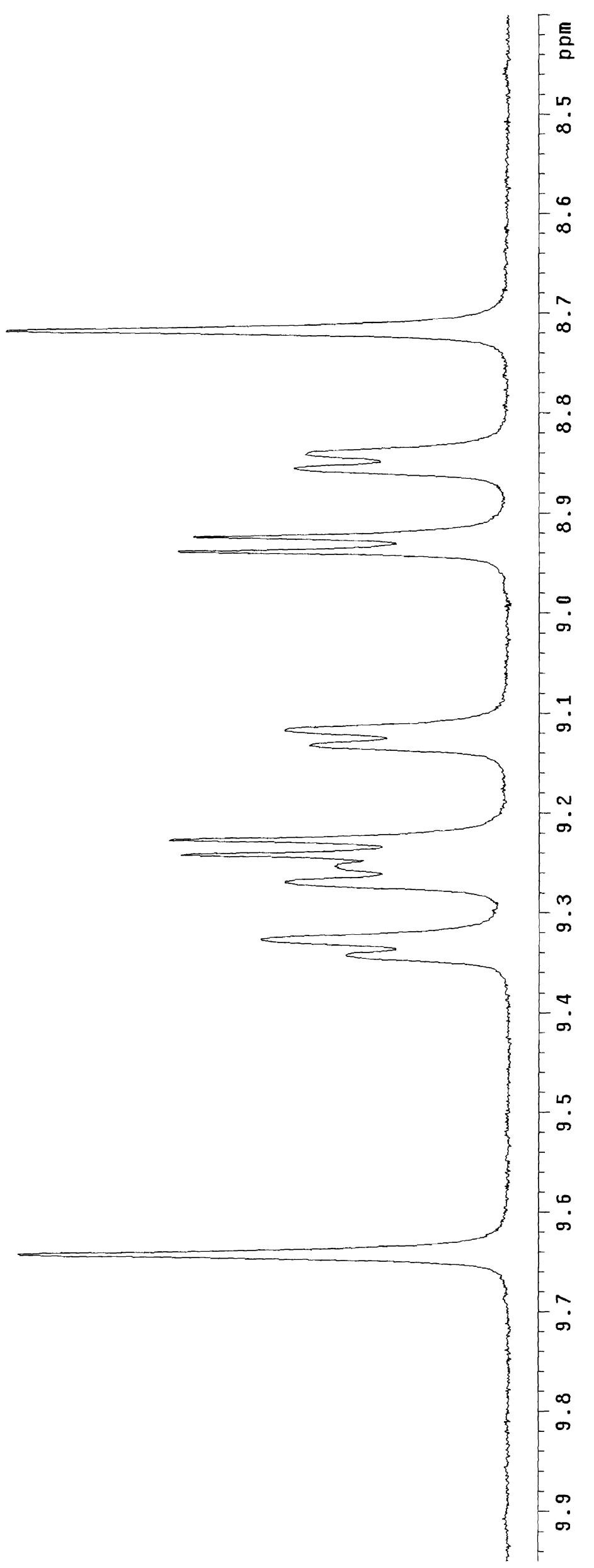




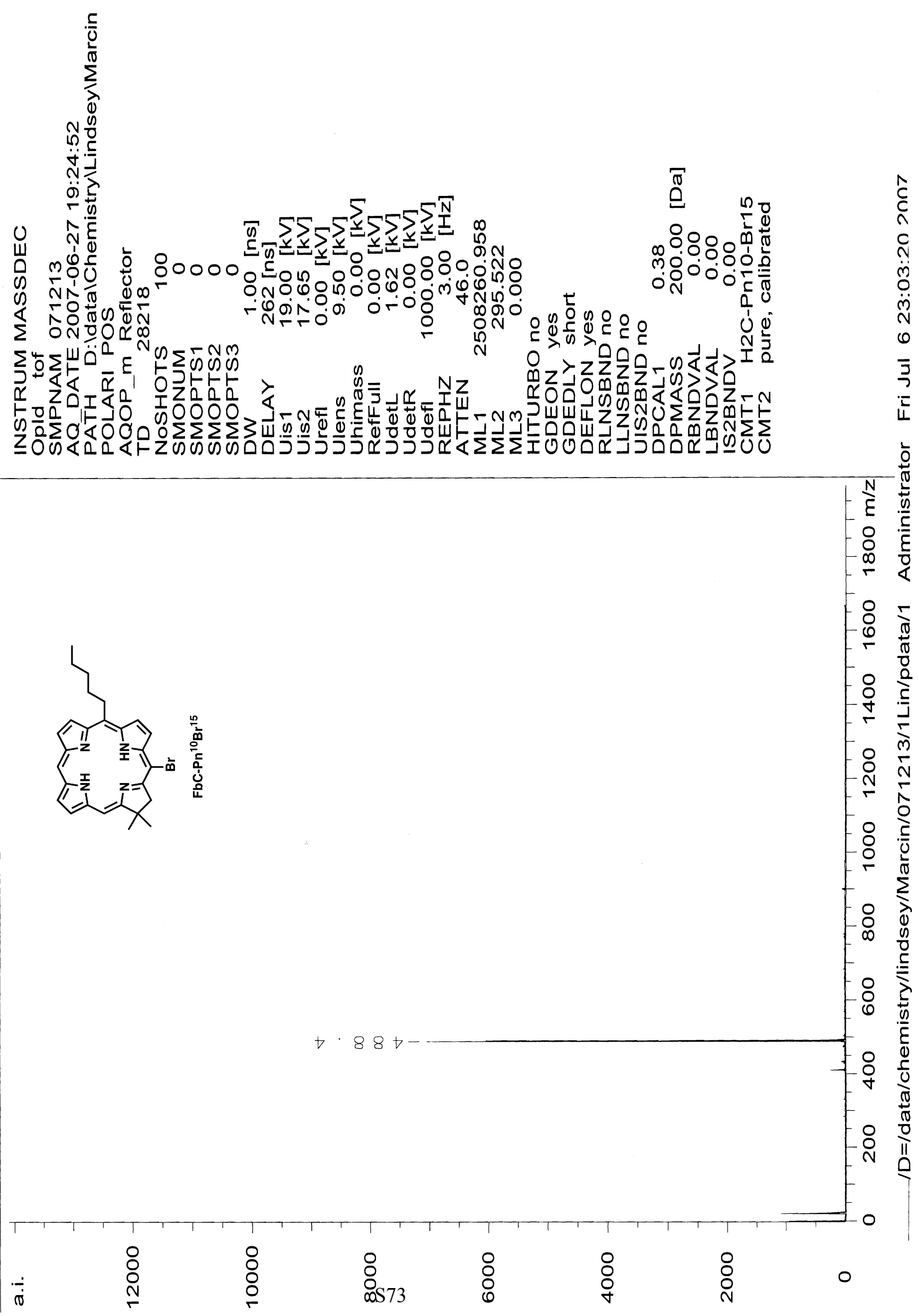



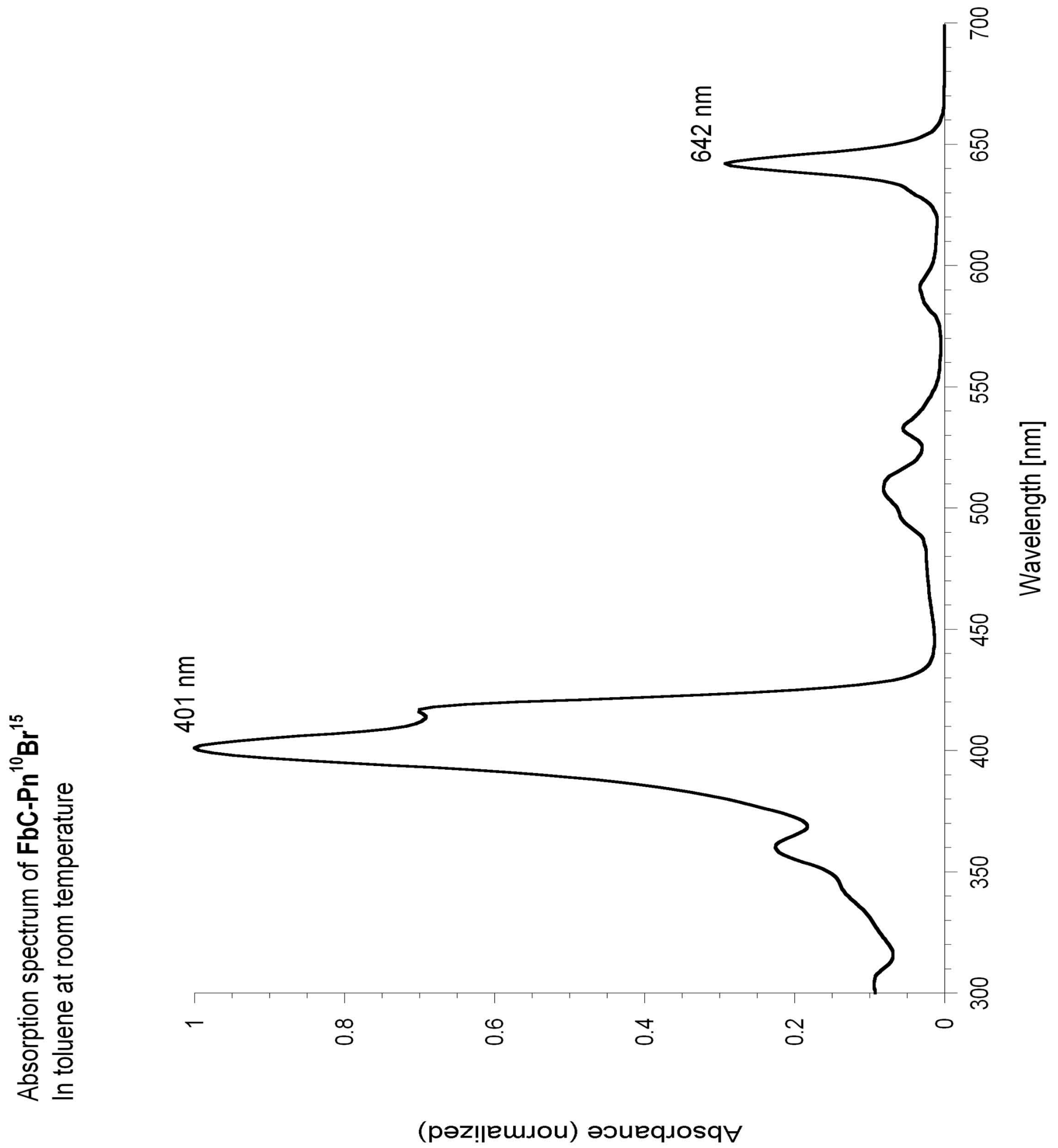


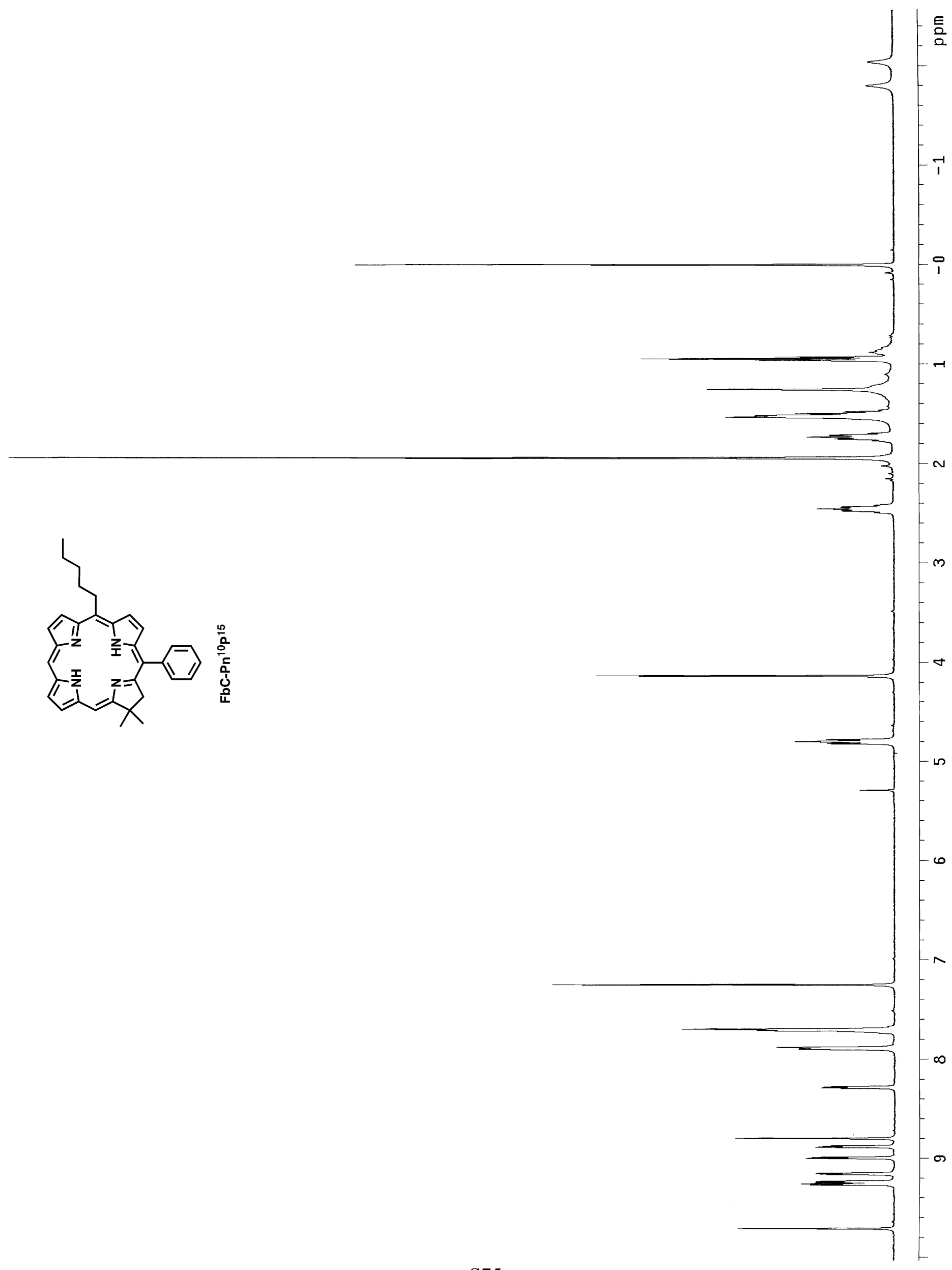




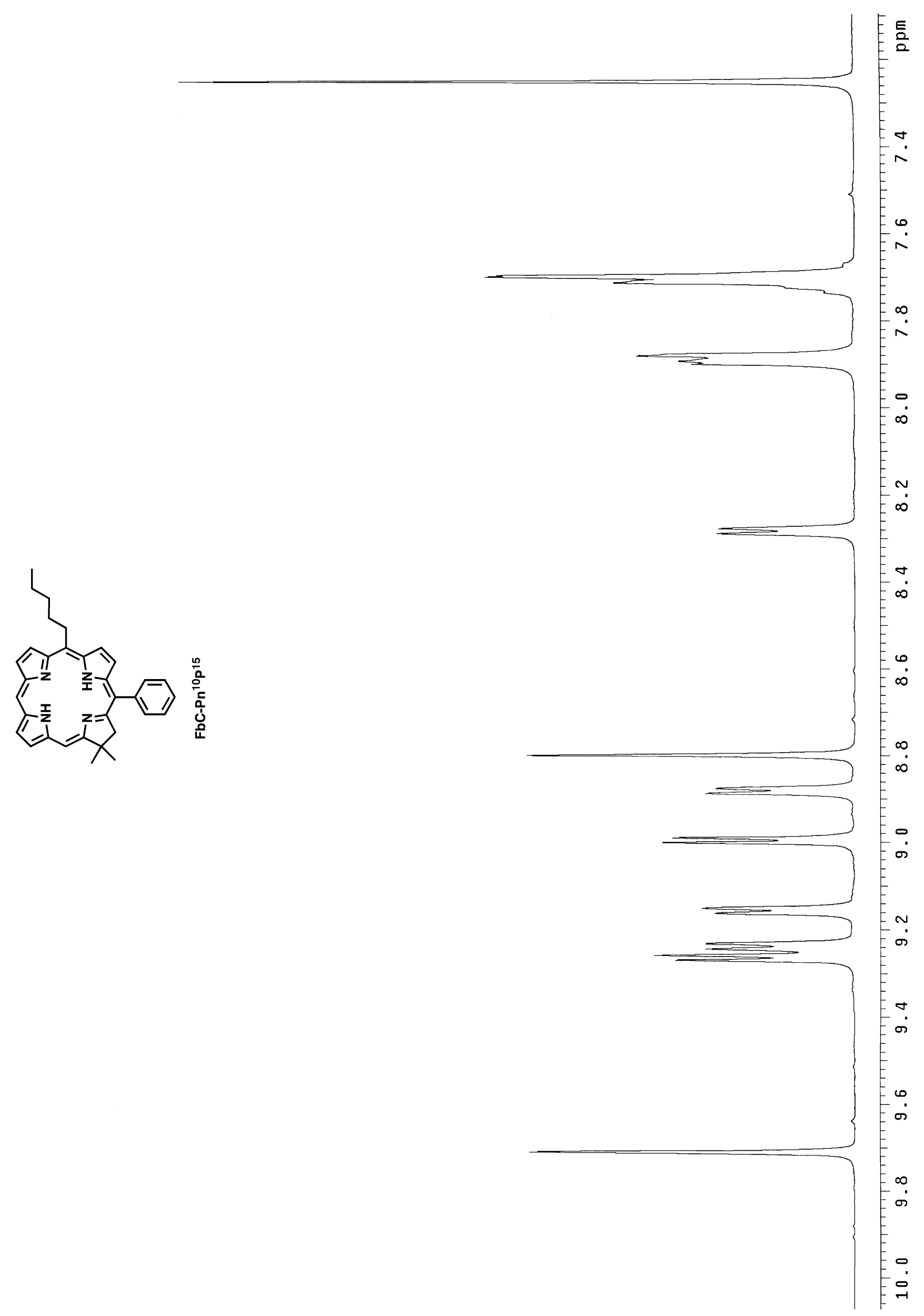




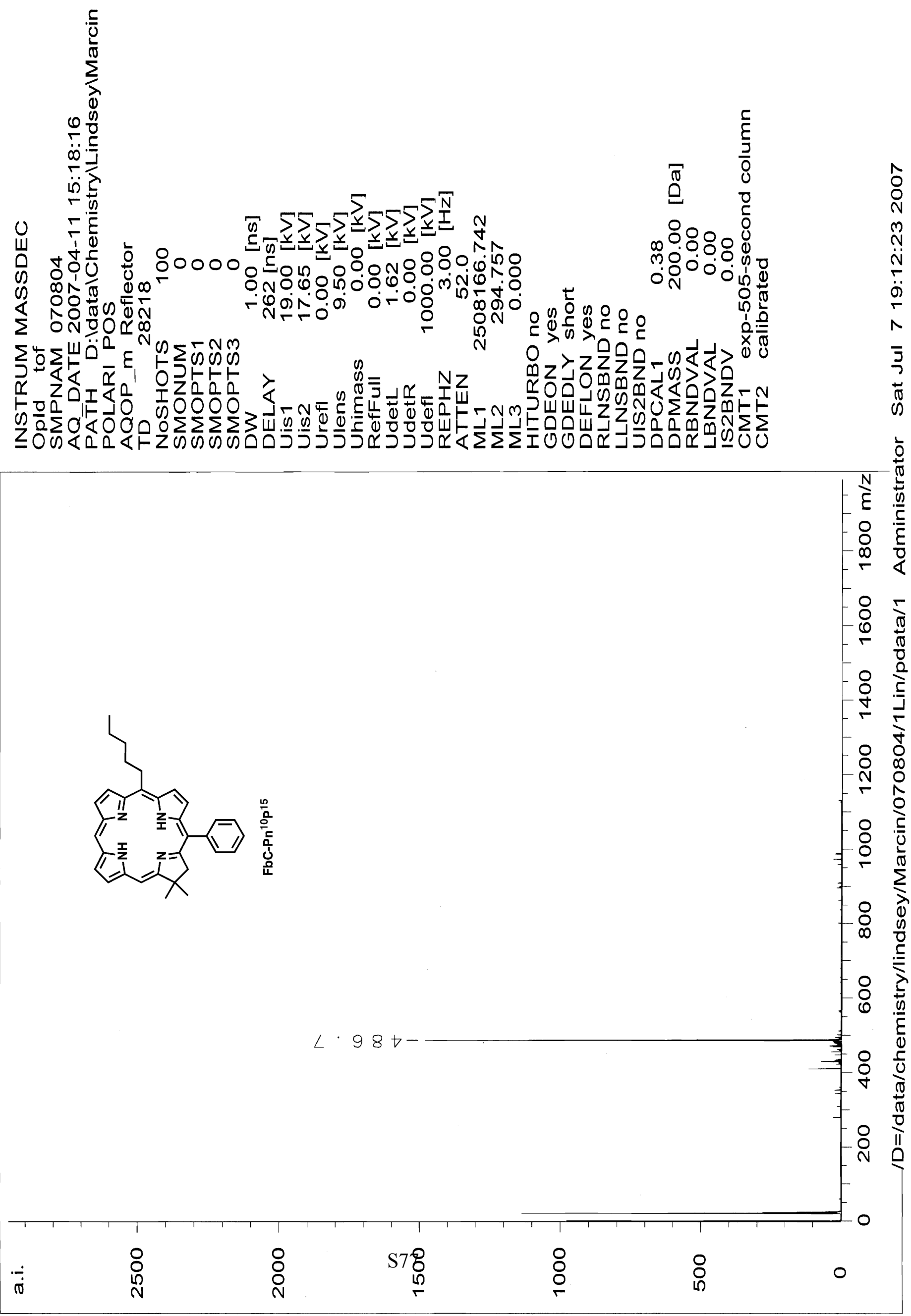



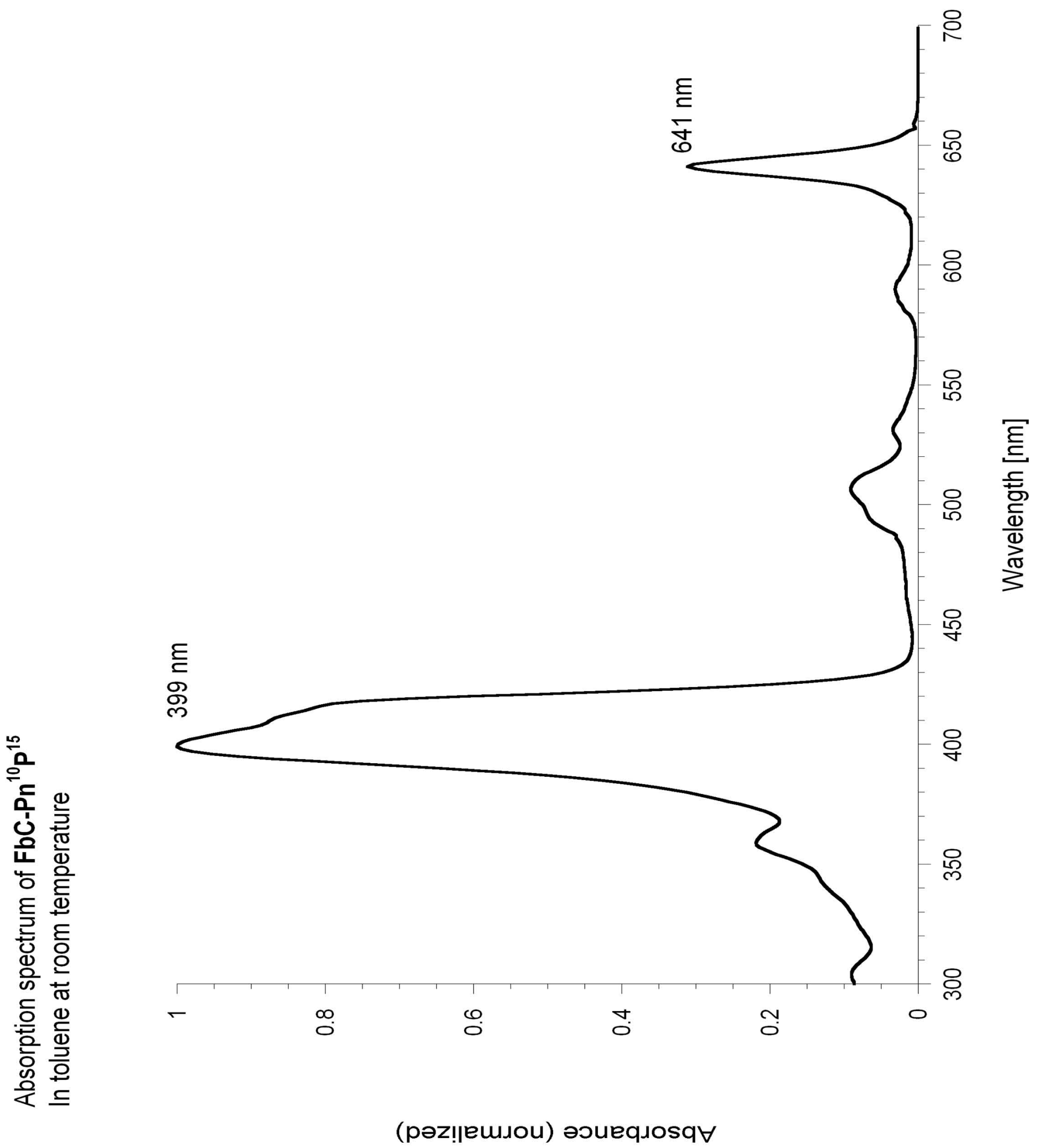


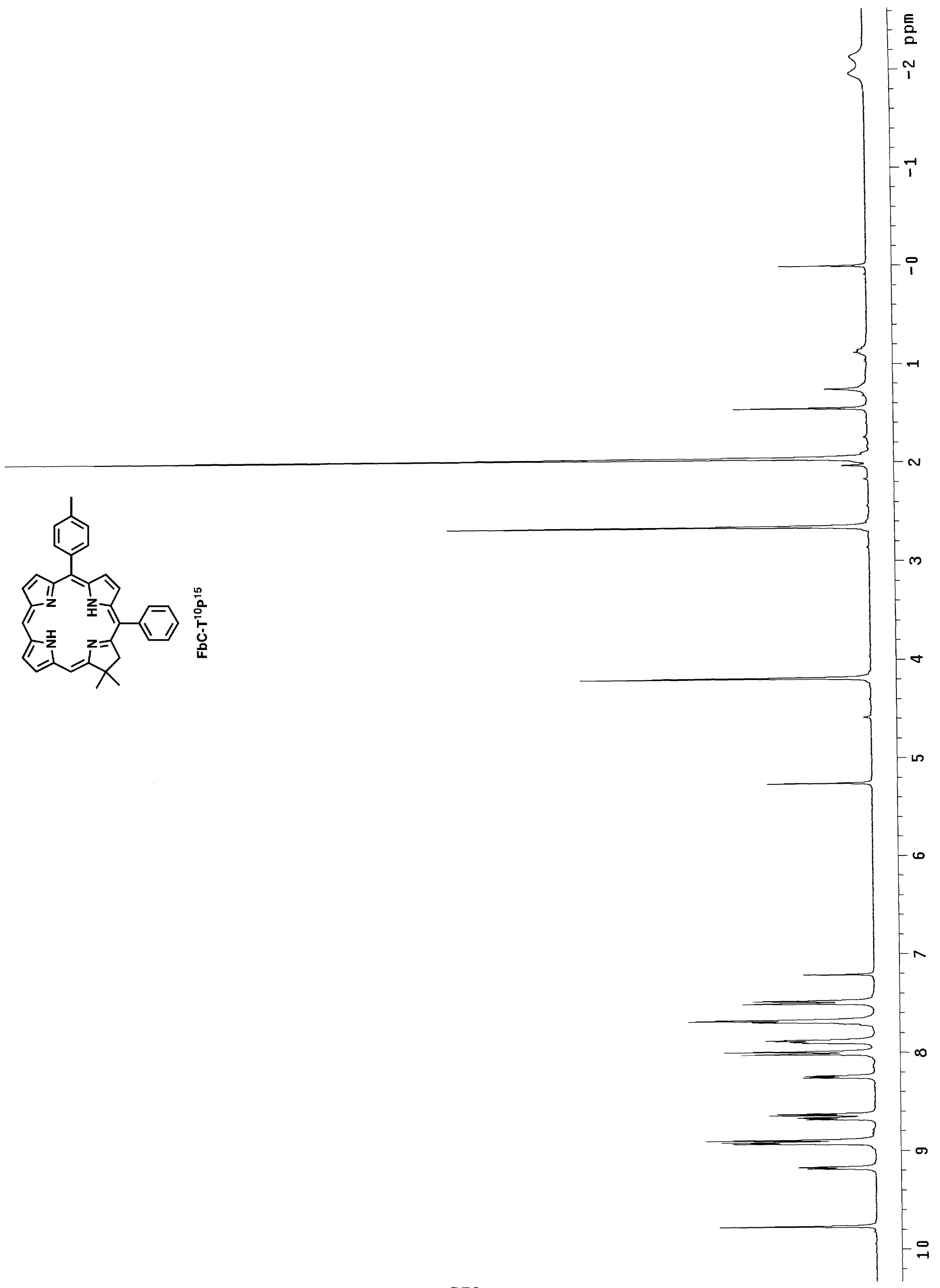




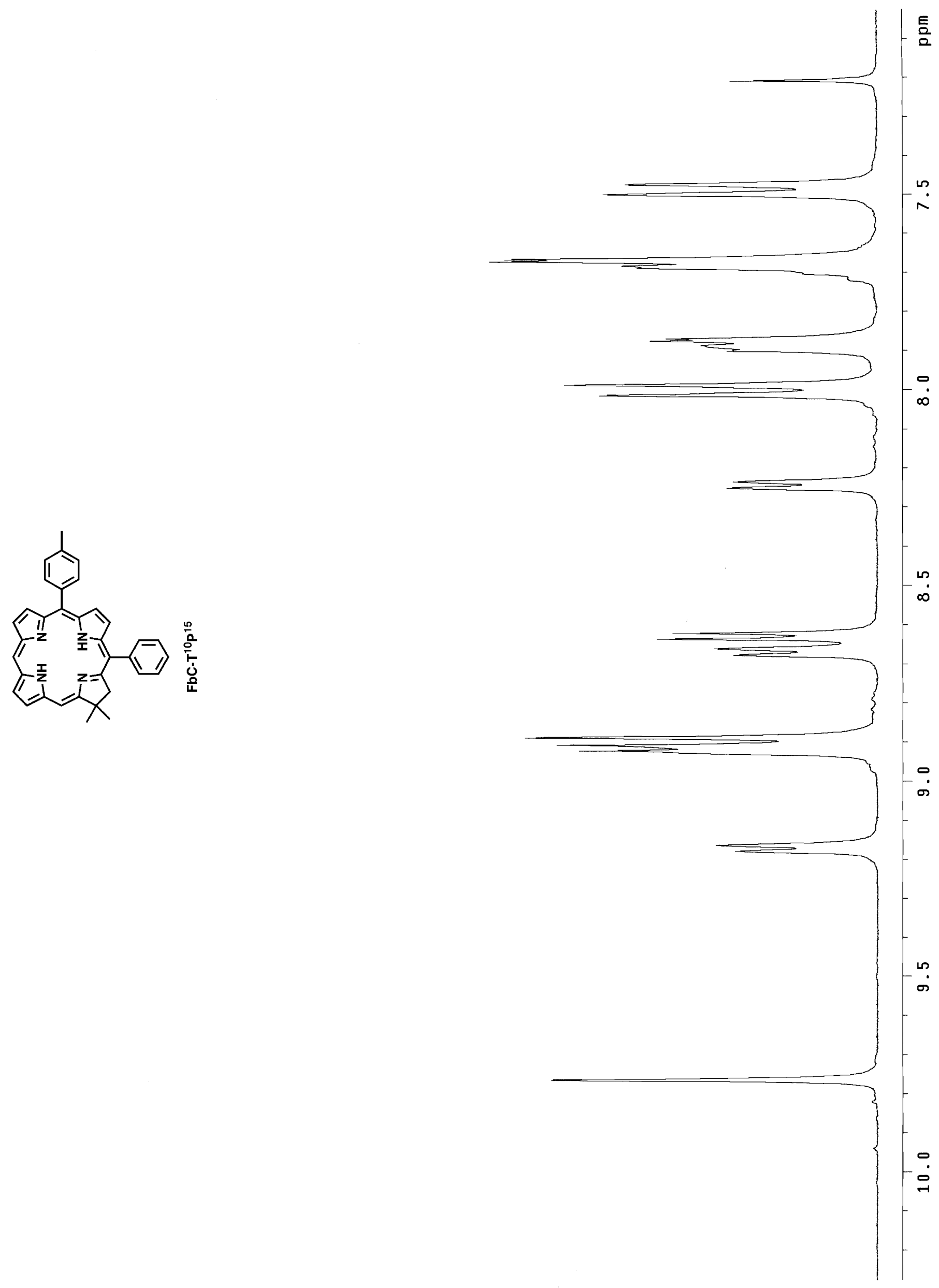



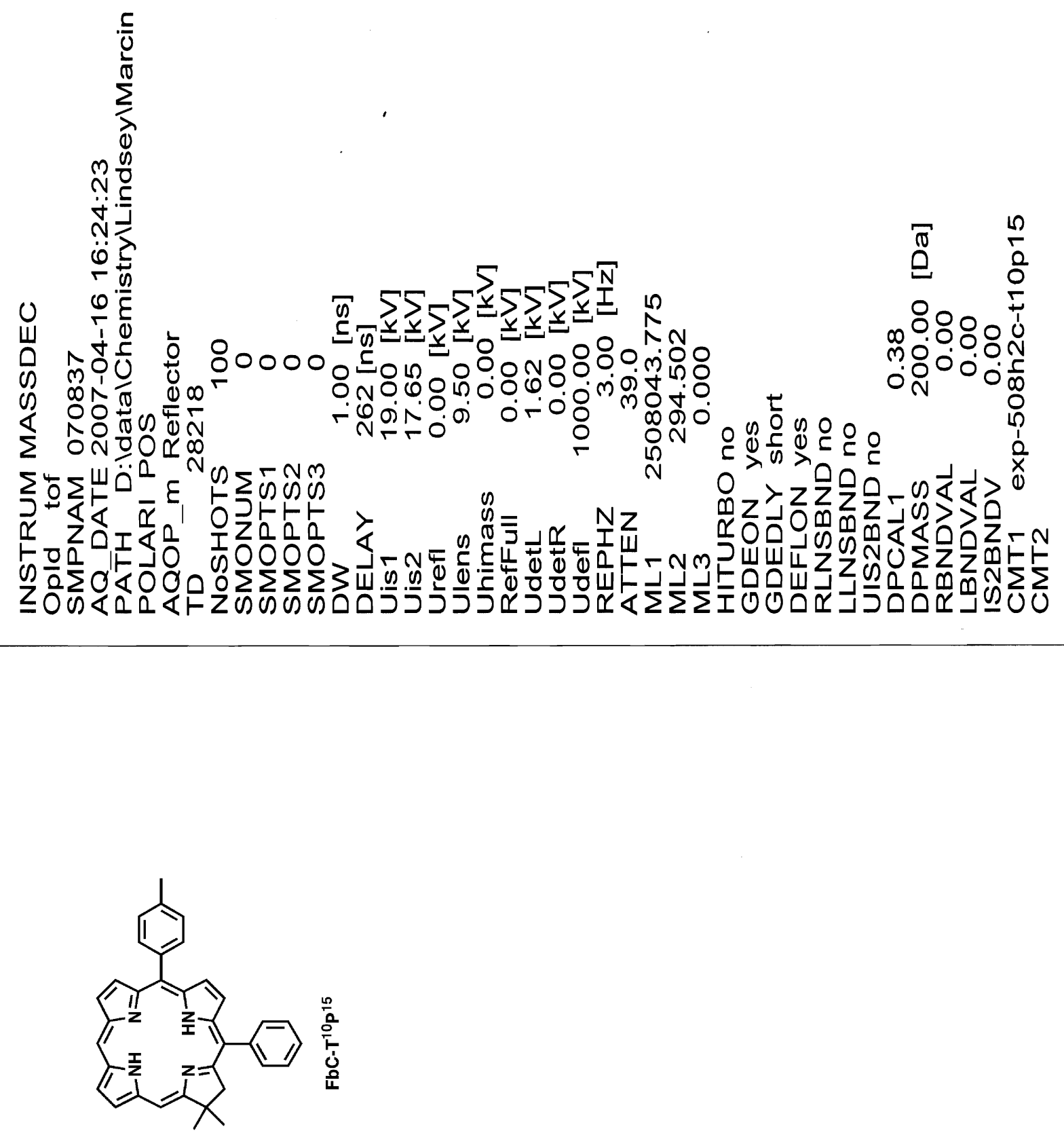

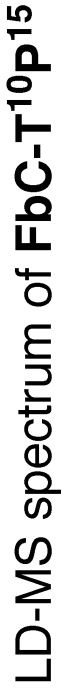




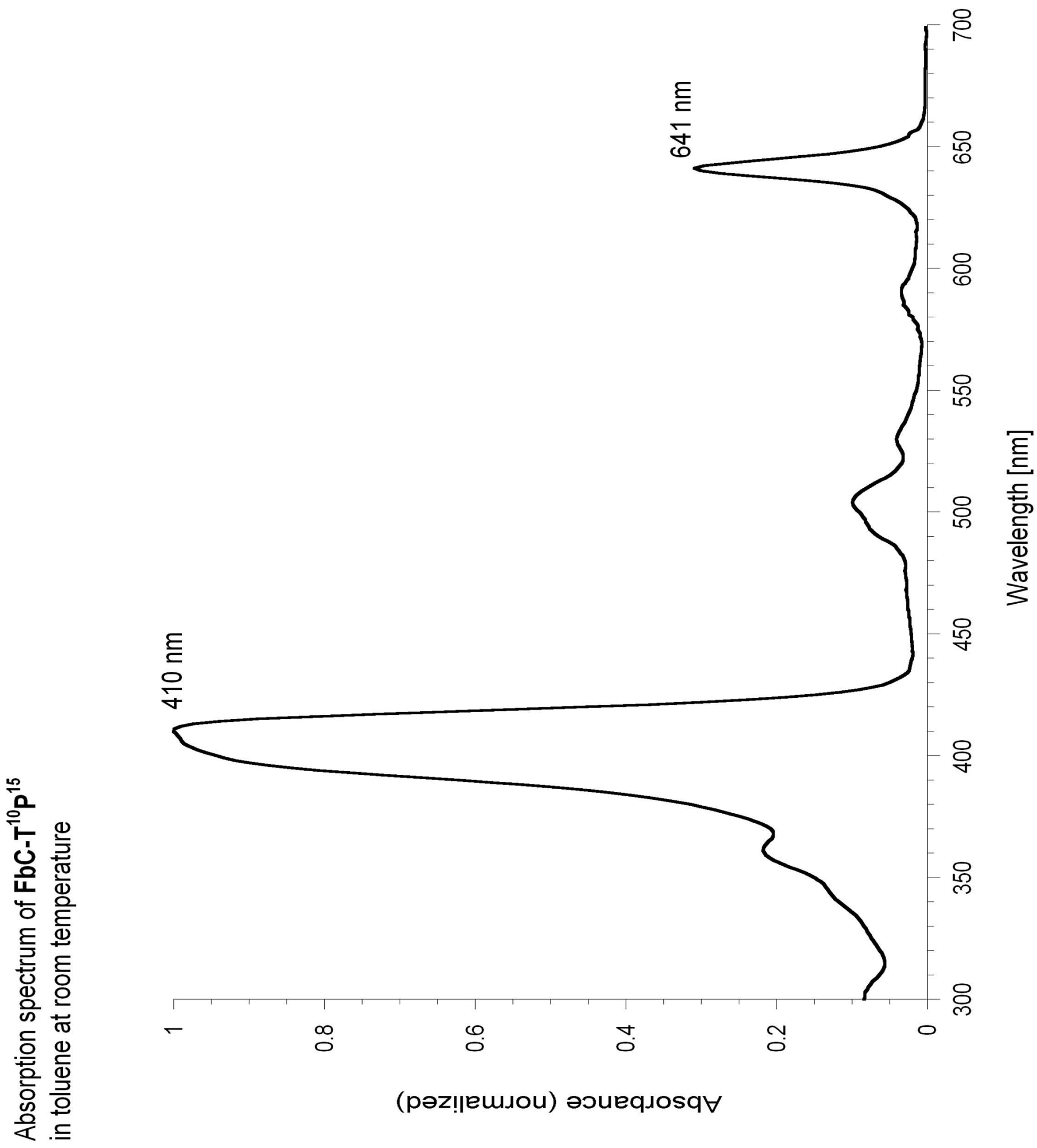




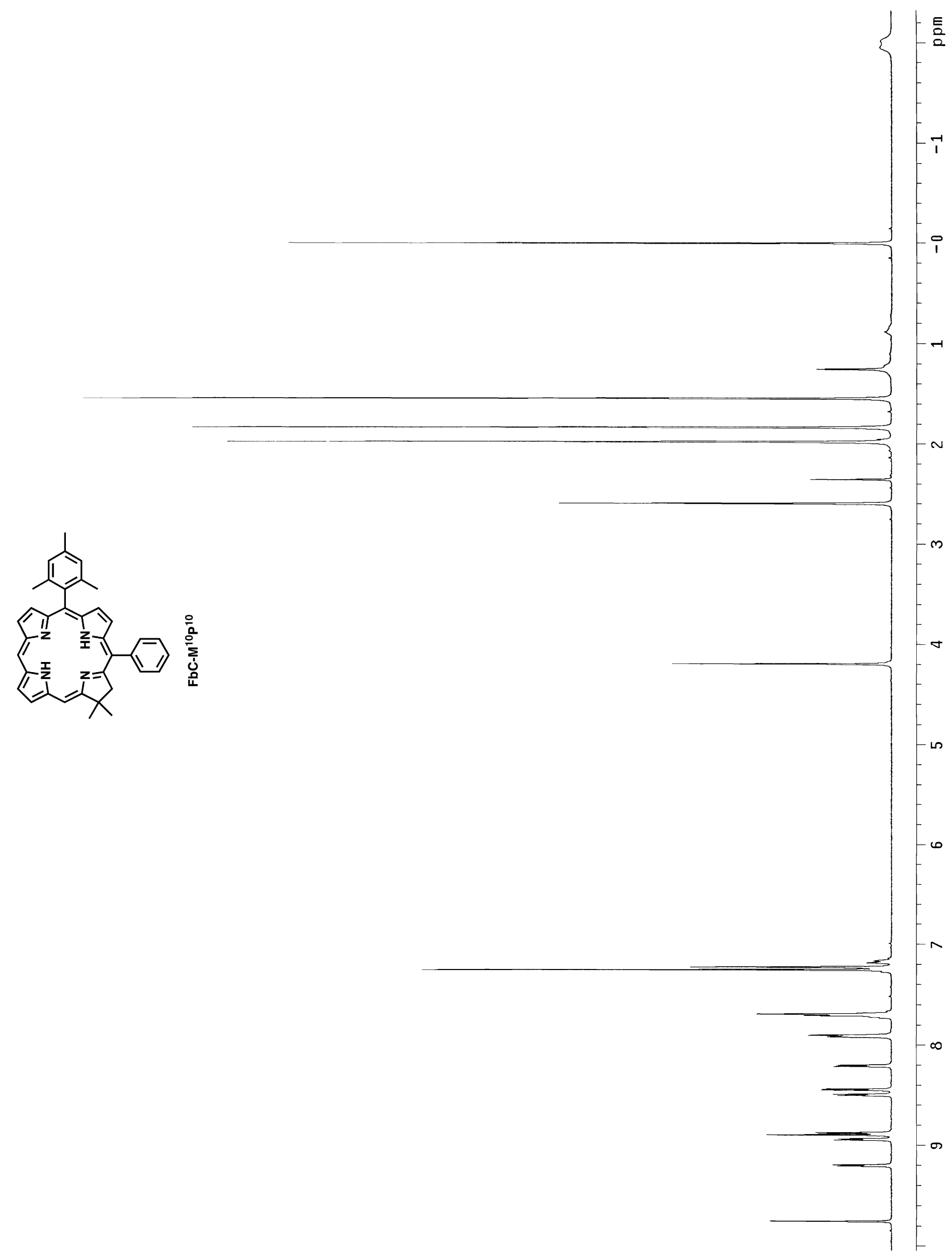




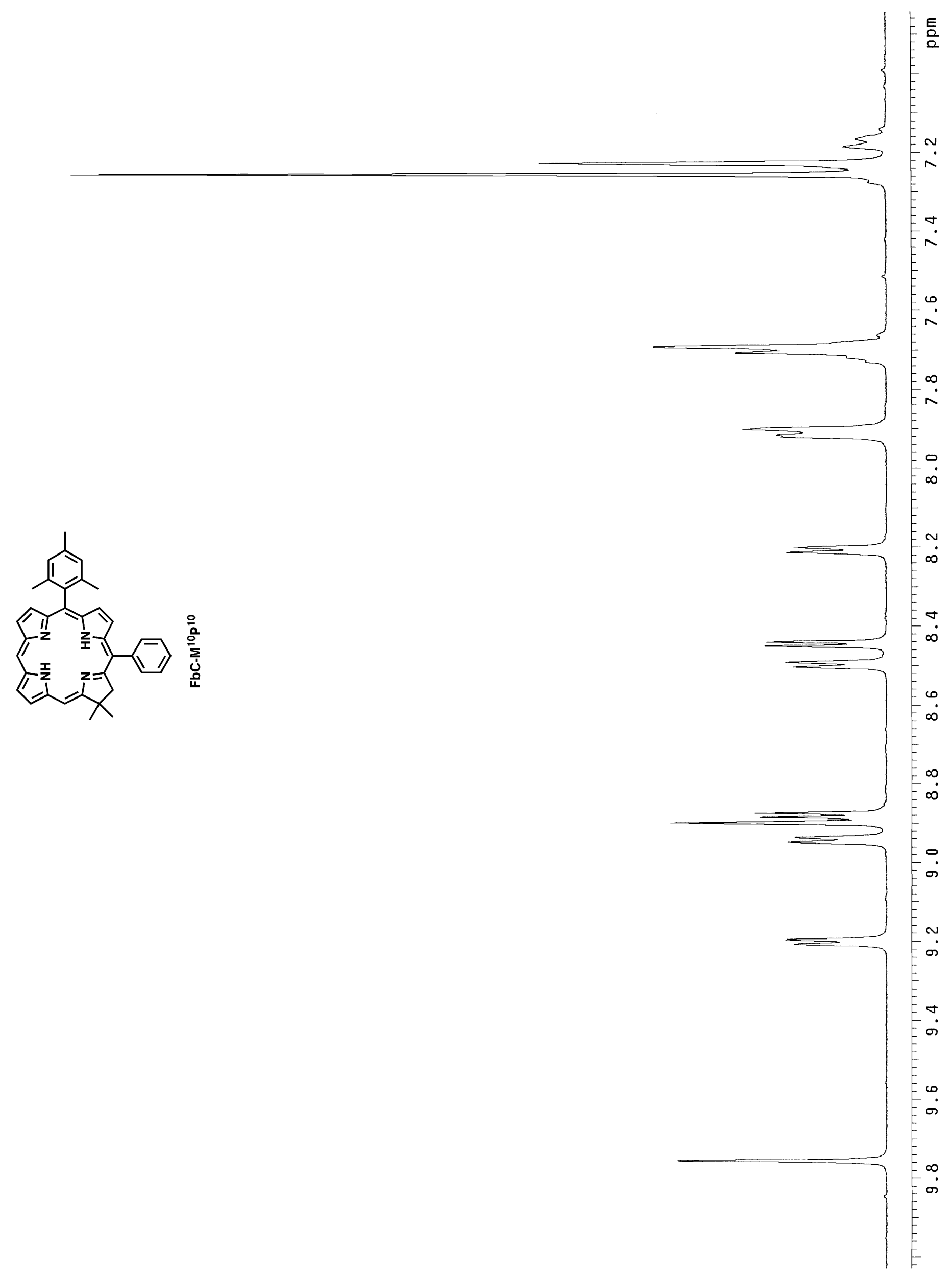




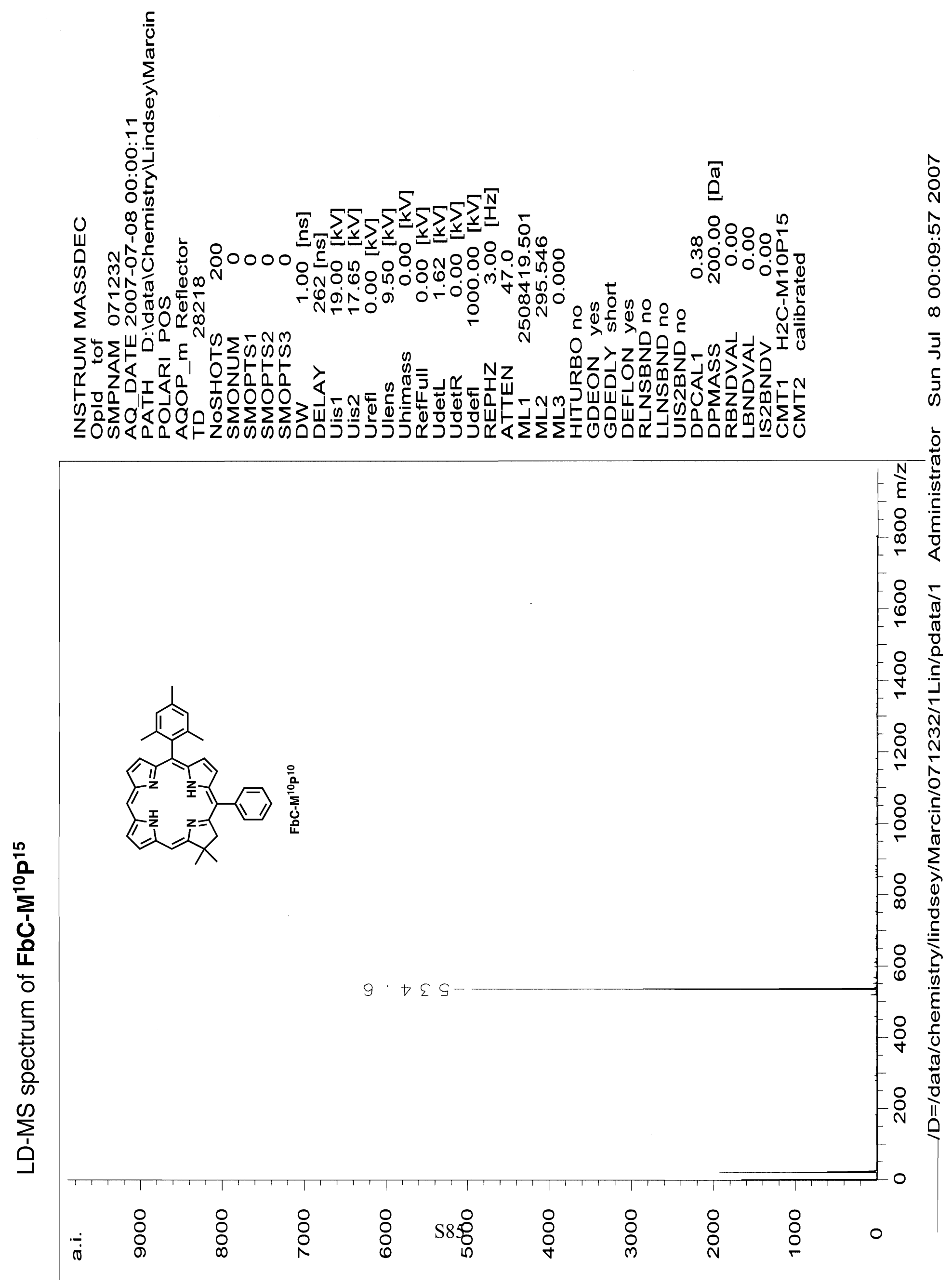




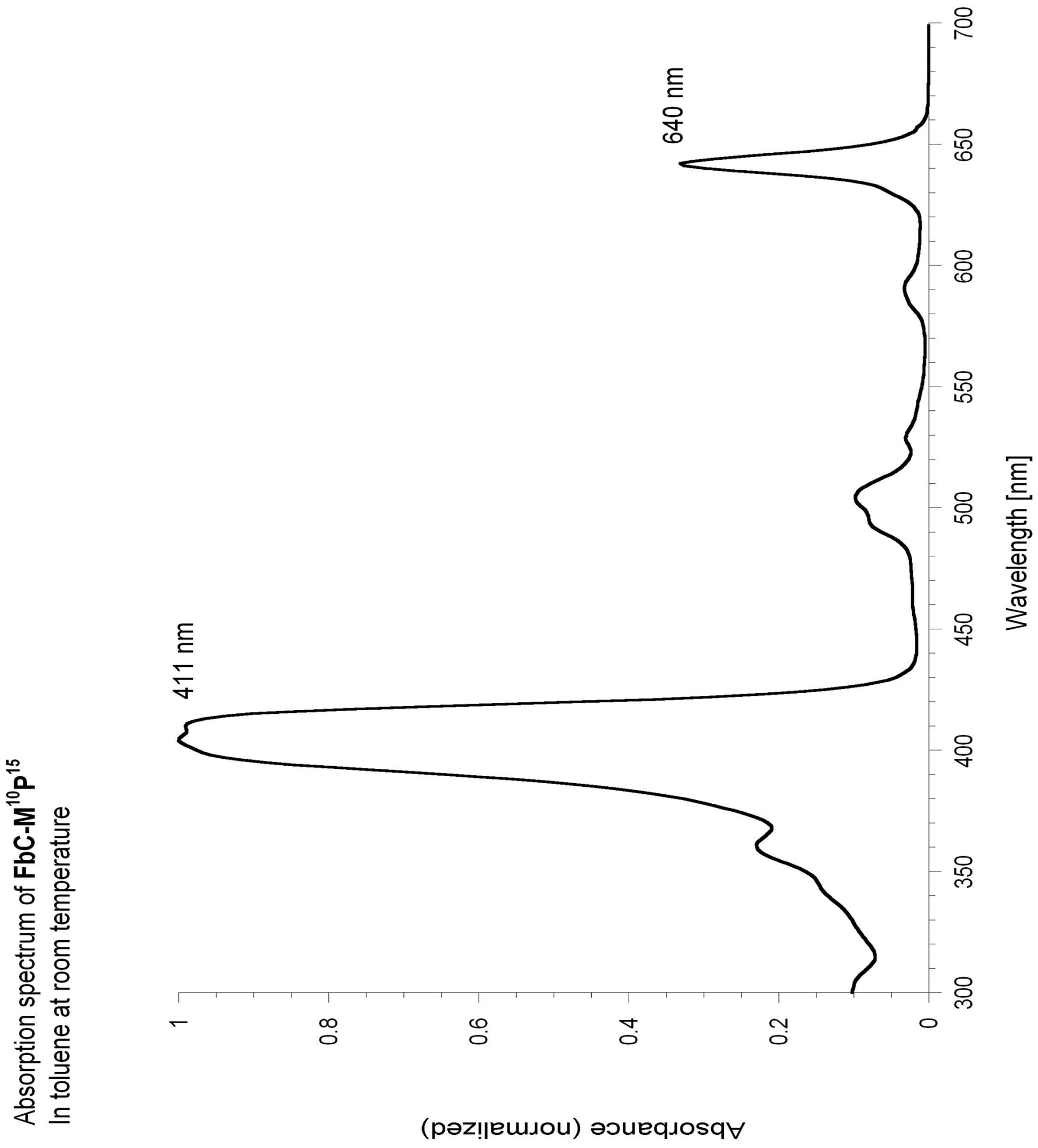




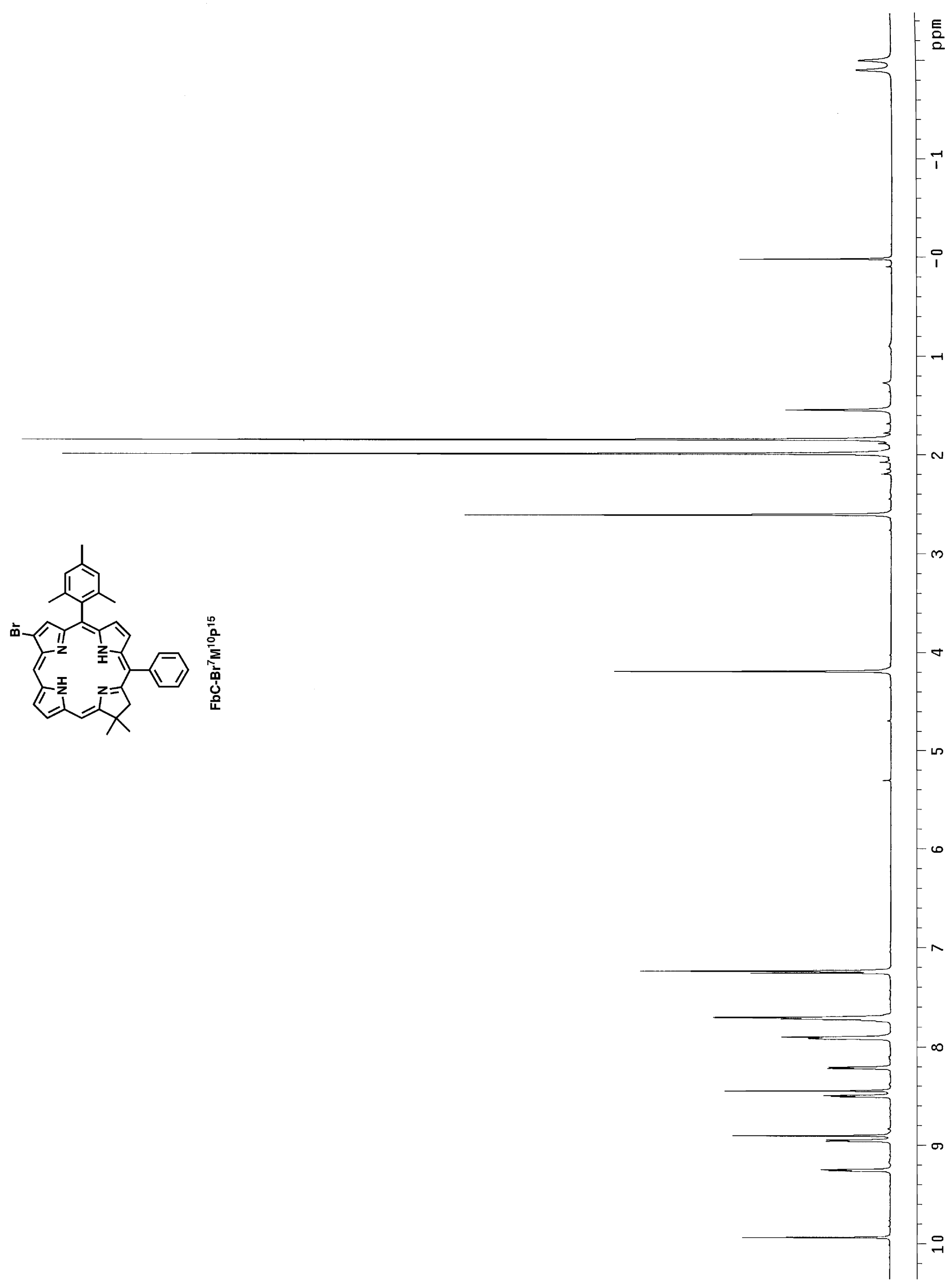




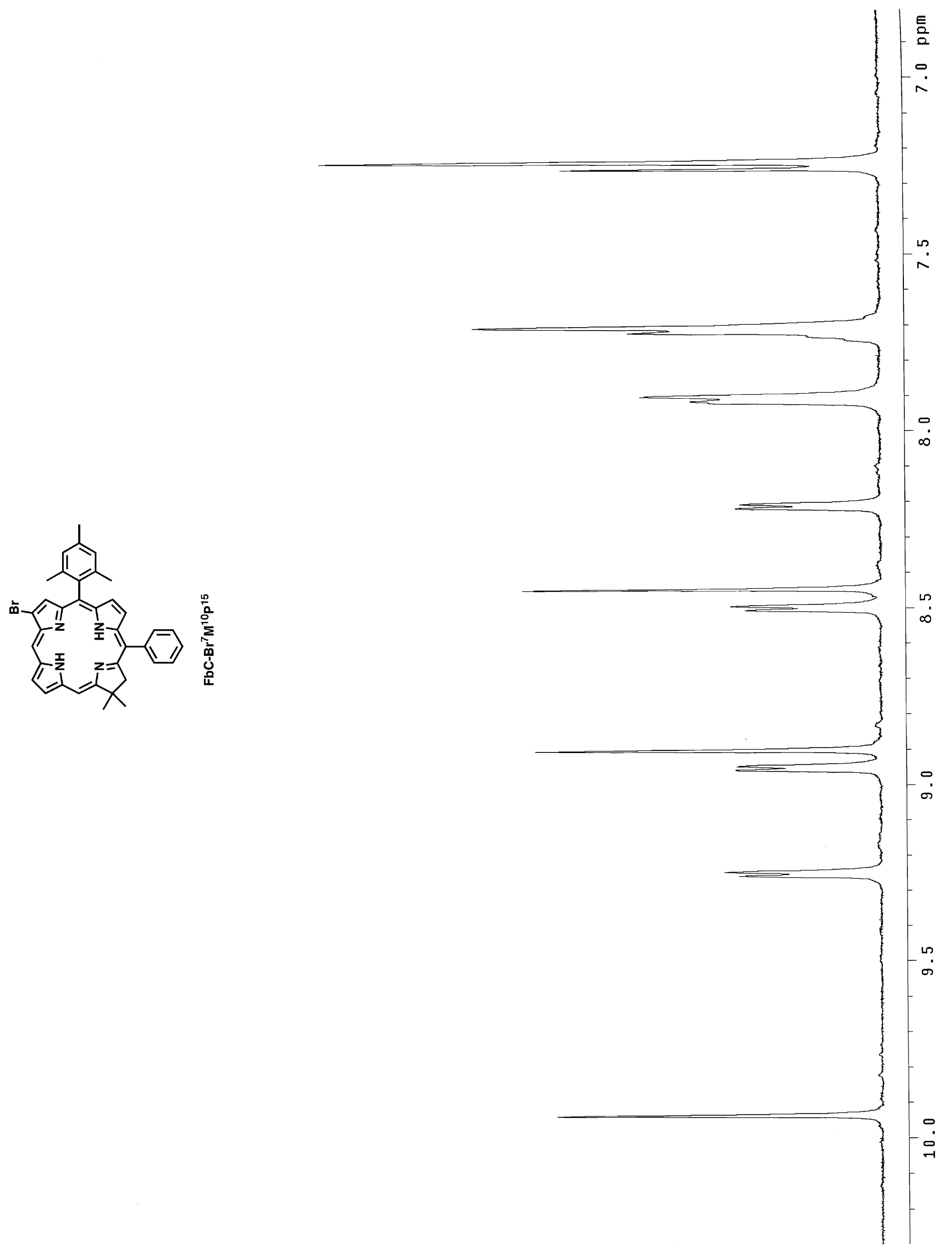




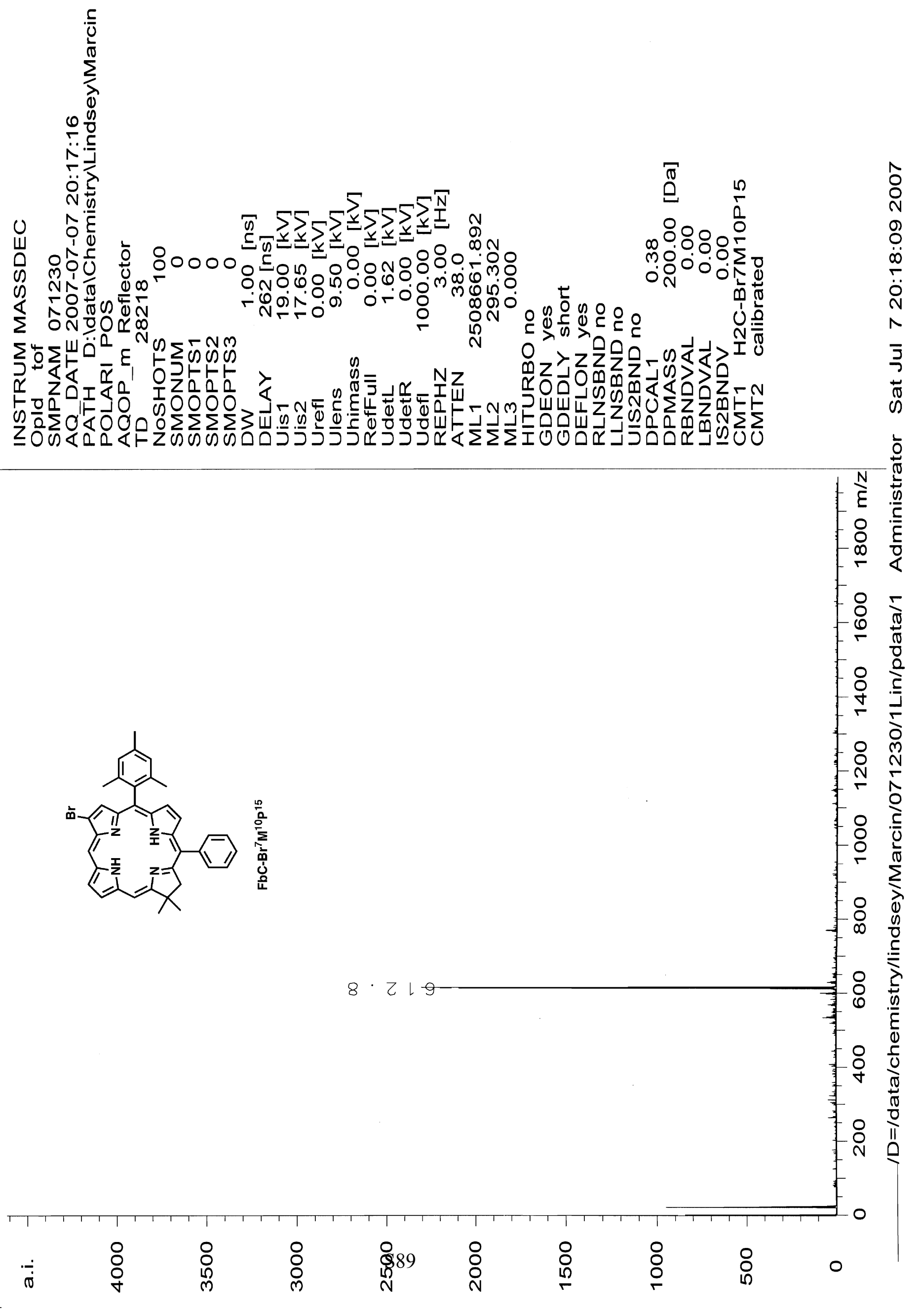




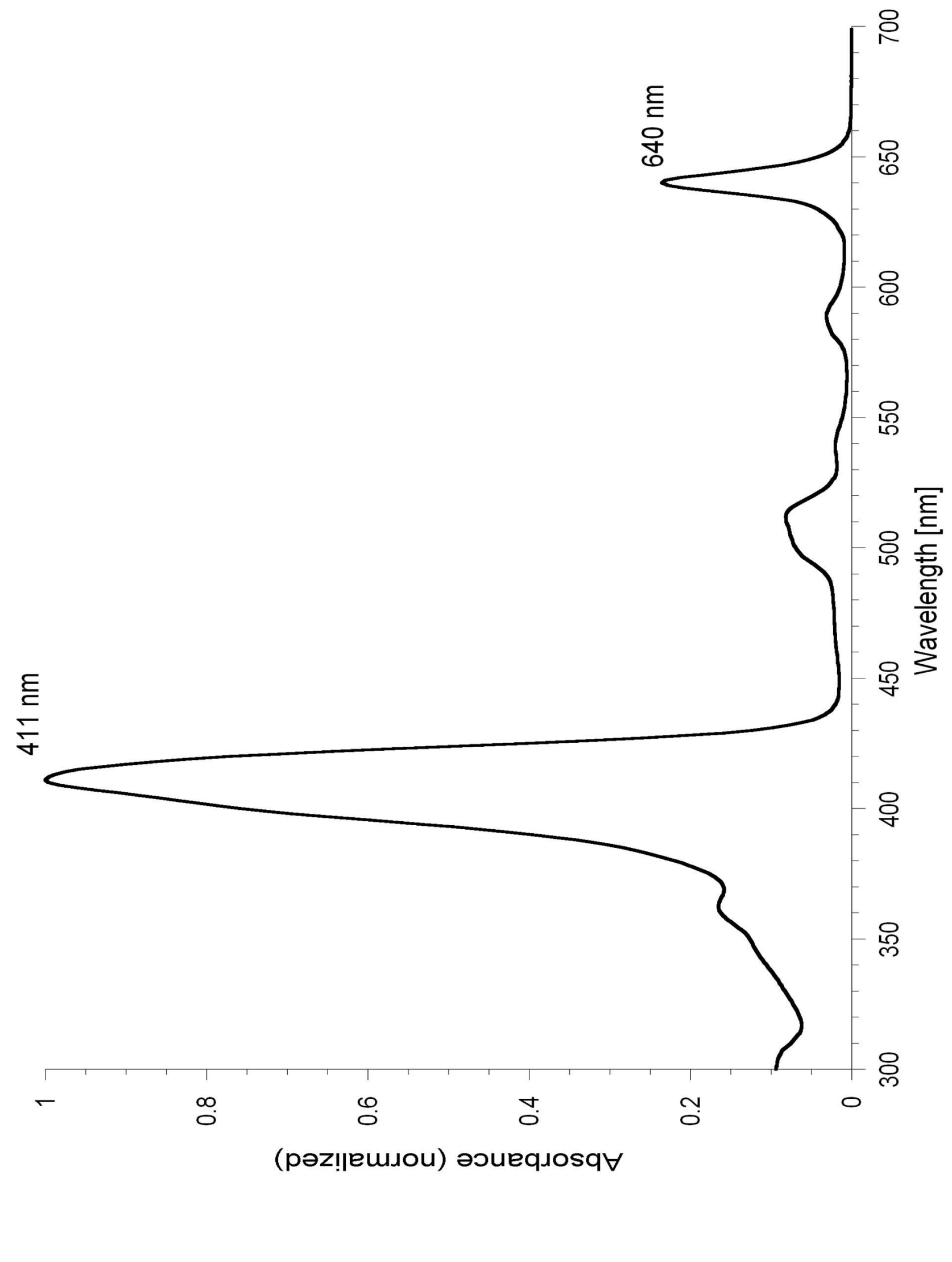




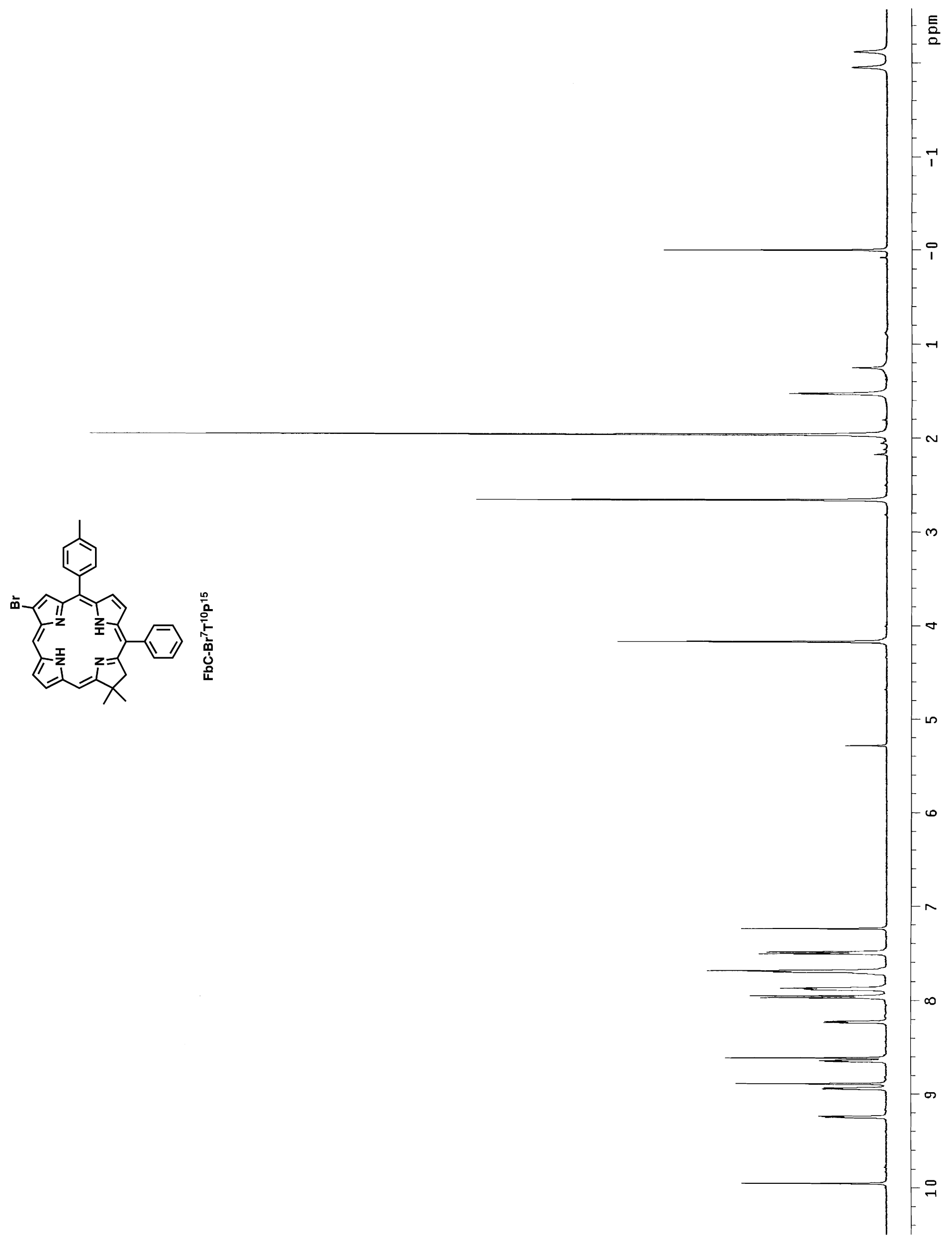




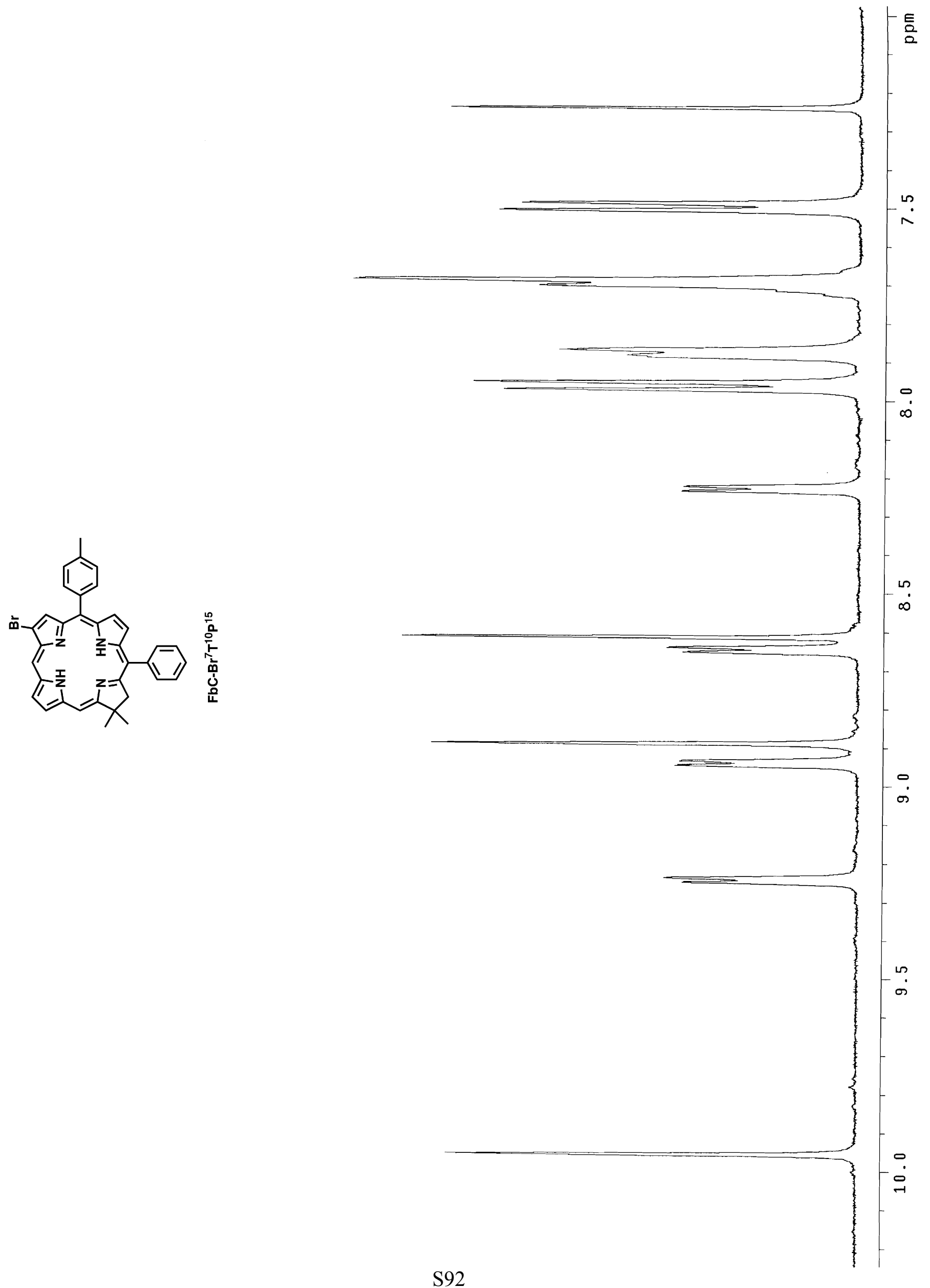




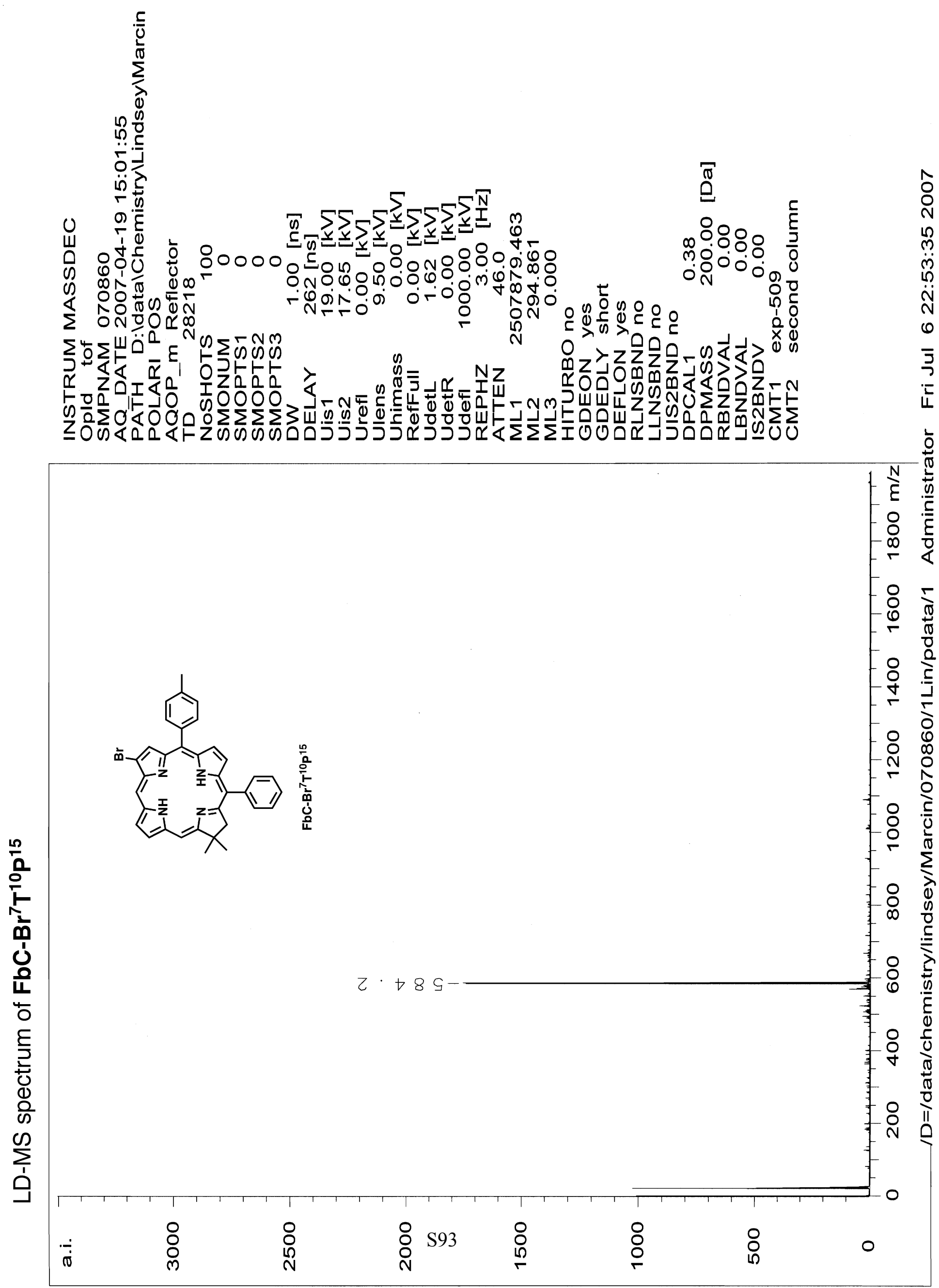



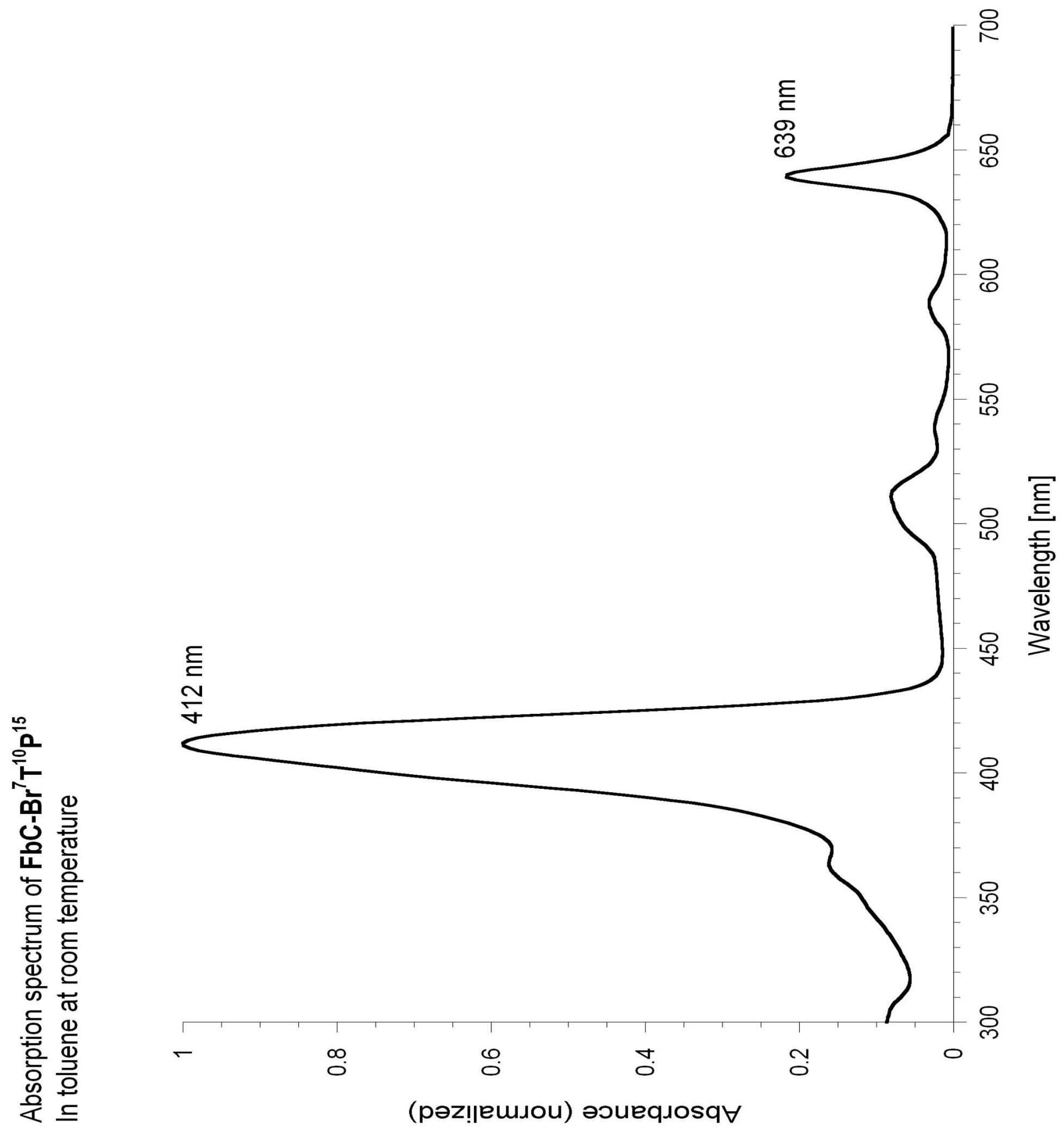


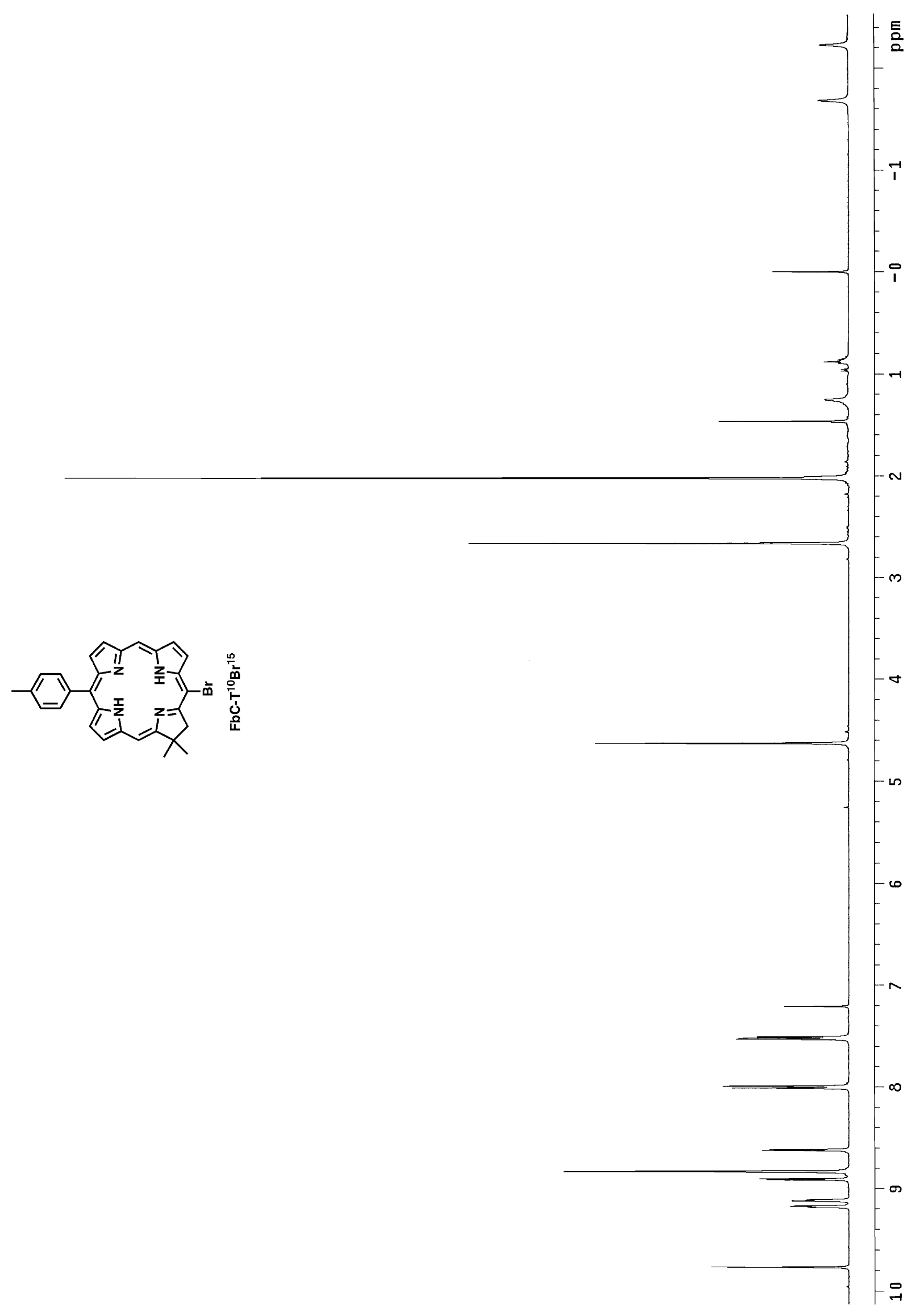




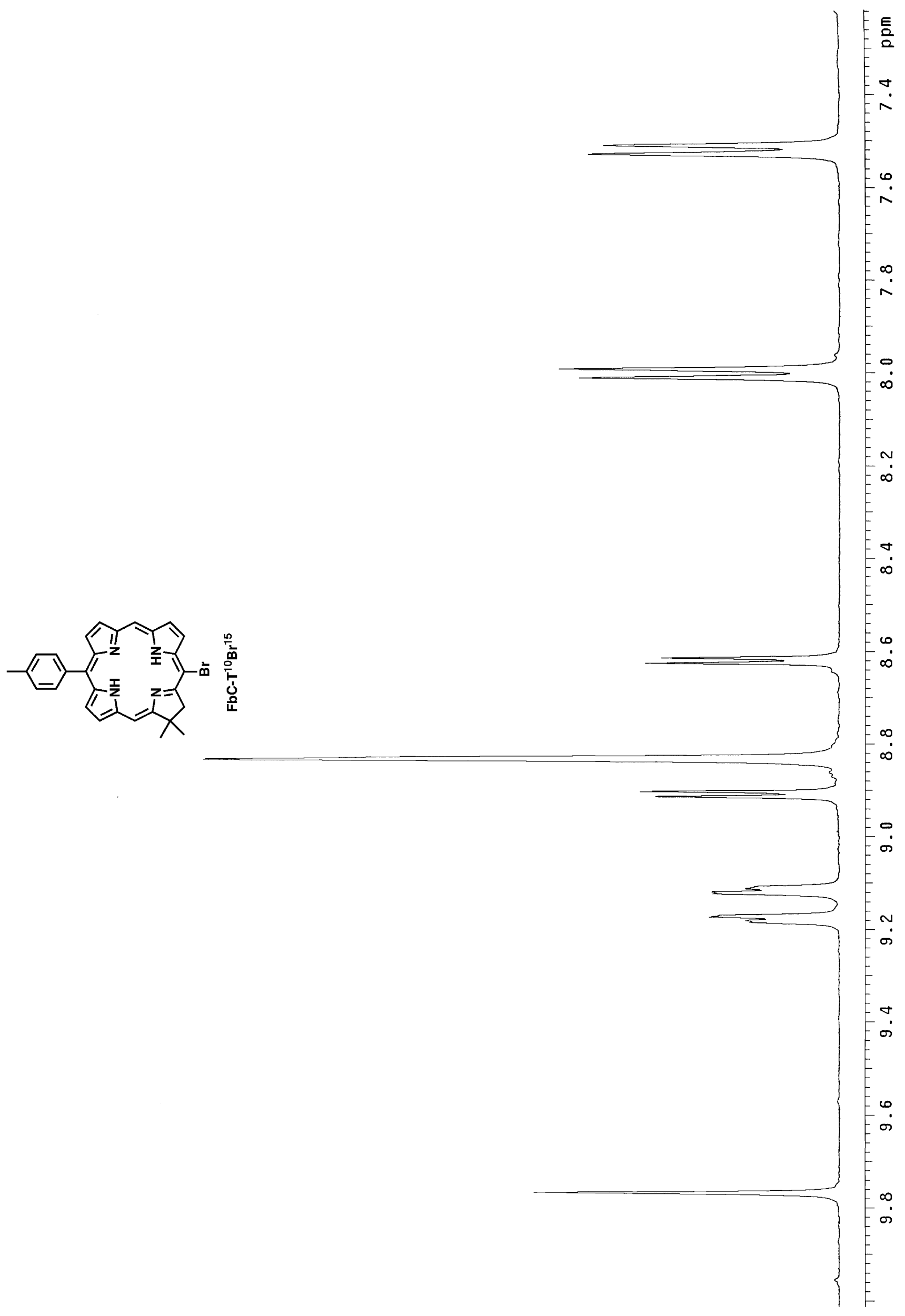




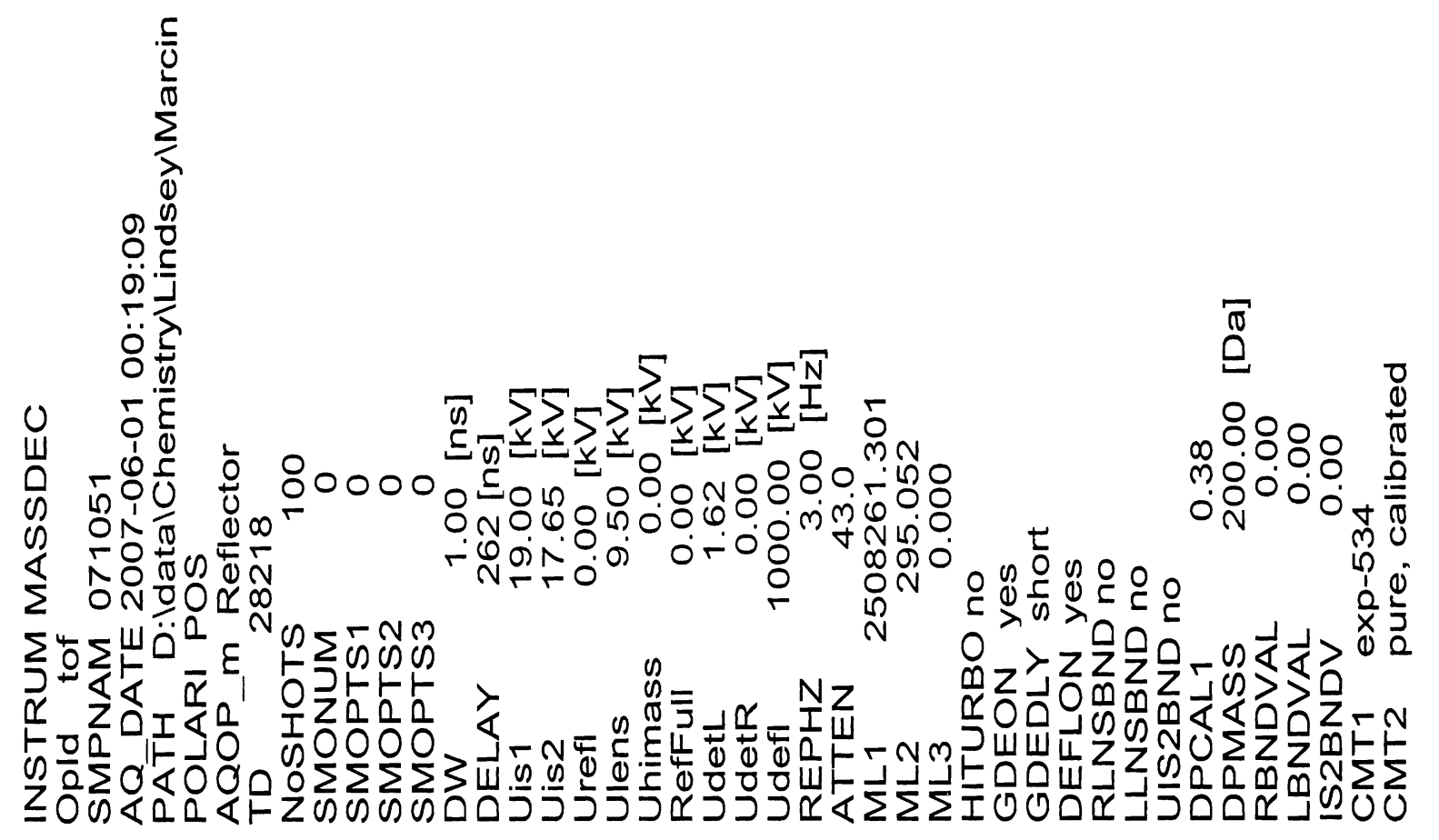

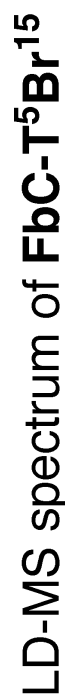

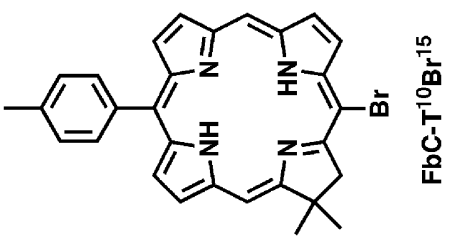

\section{5}

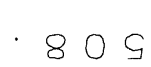



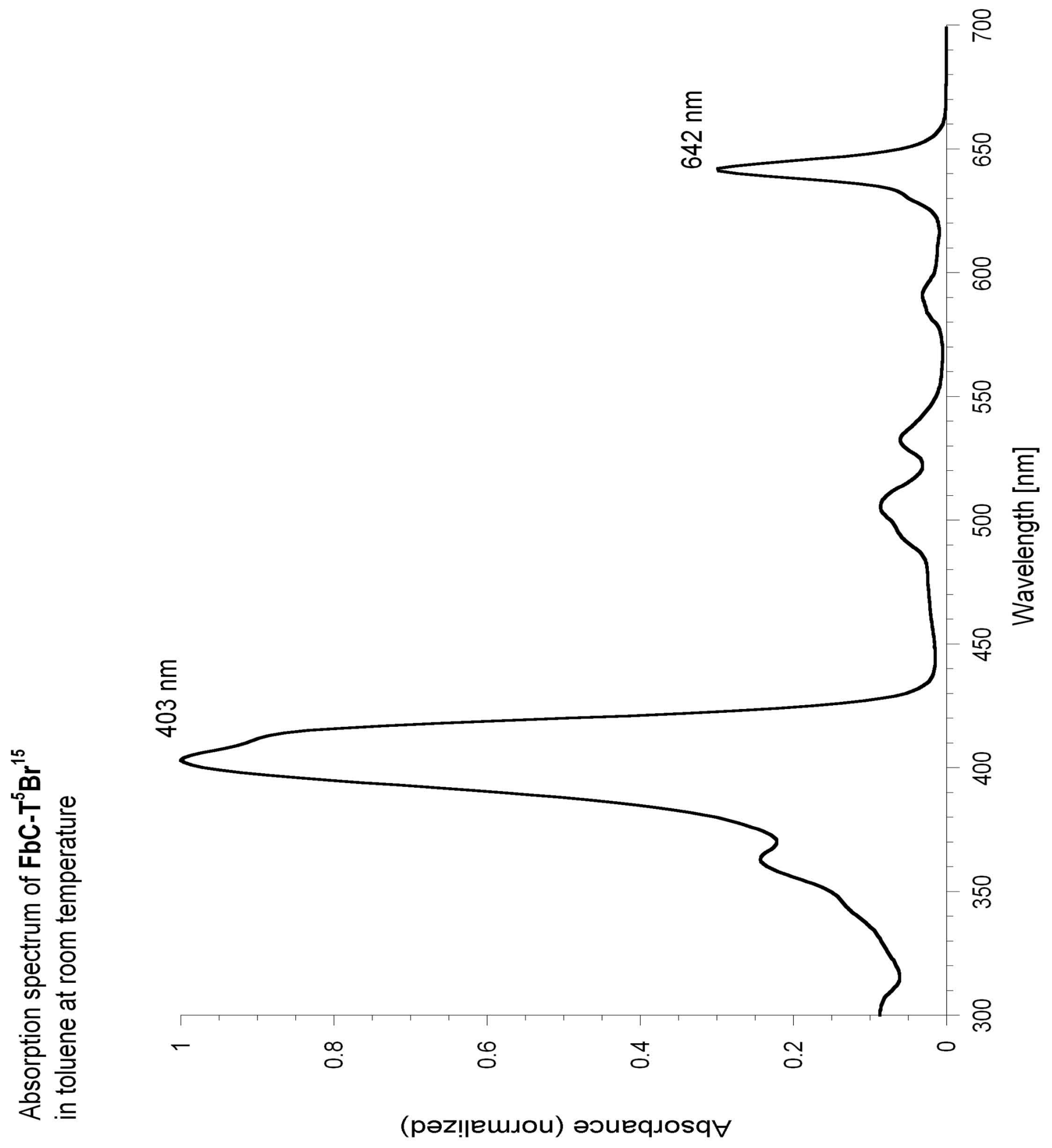


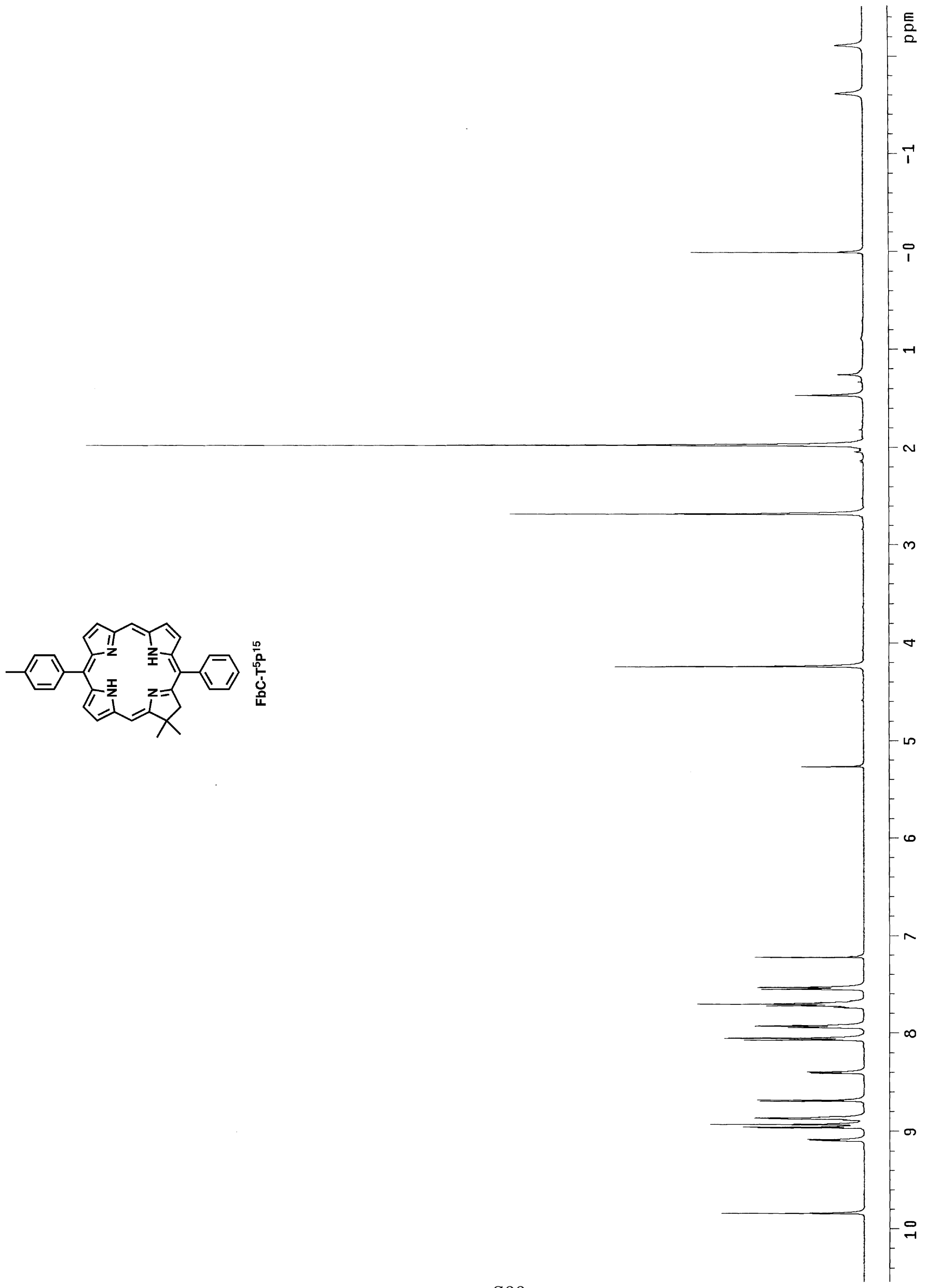




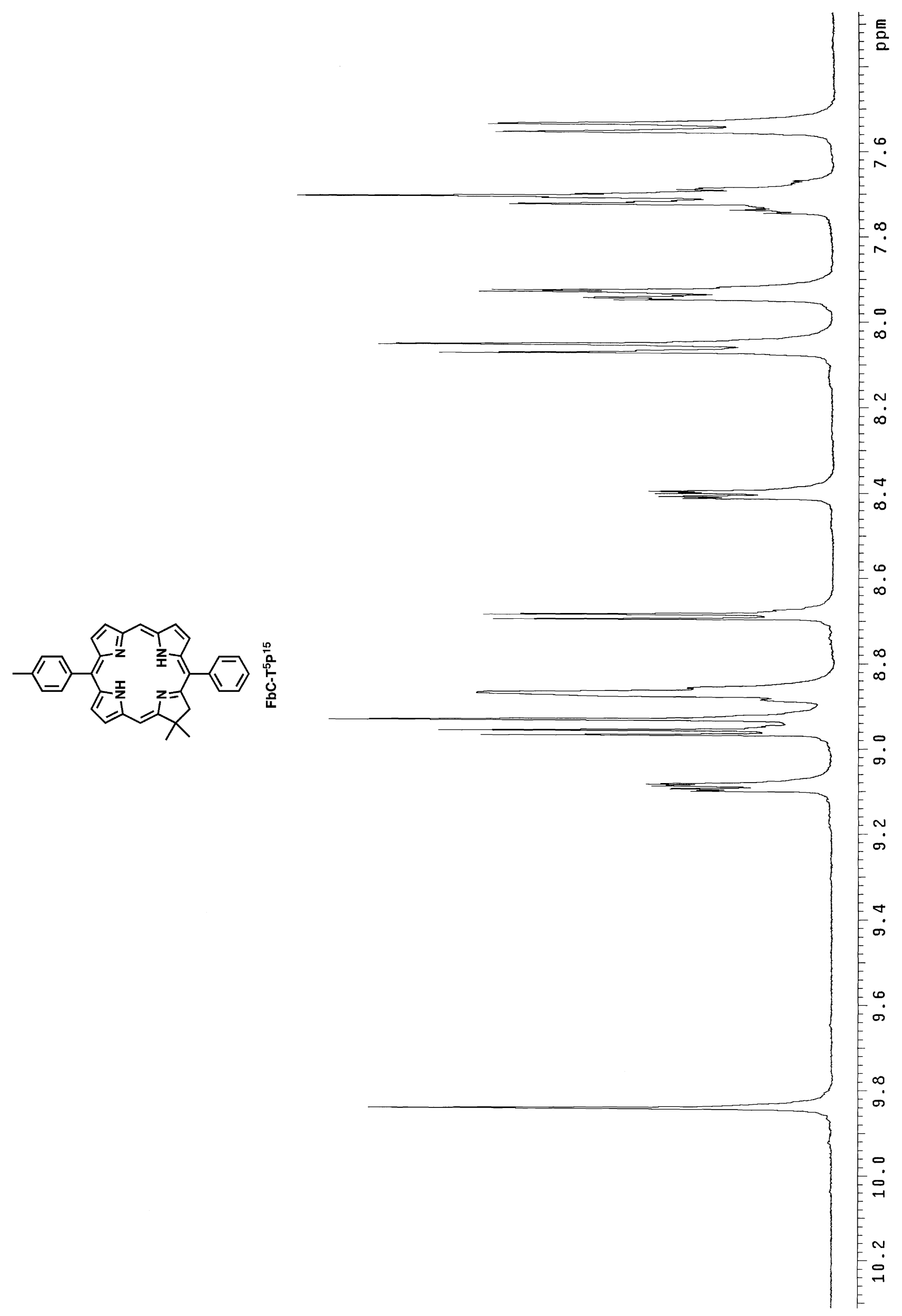




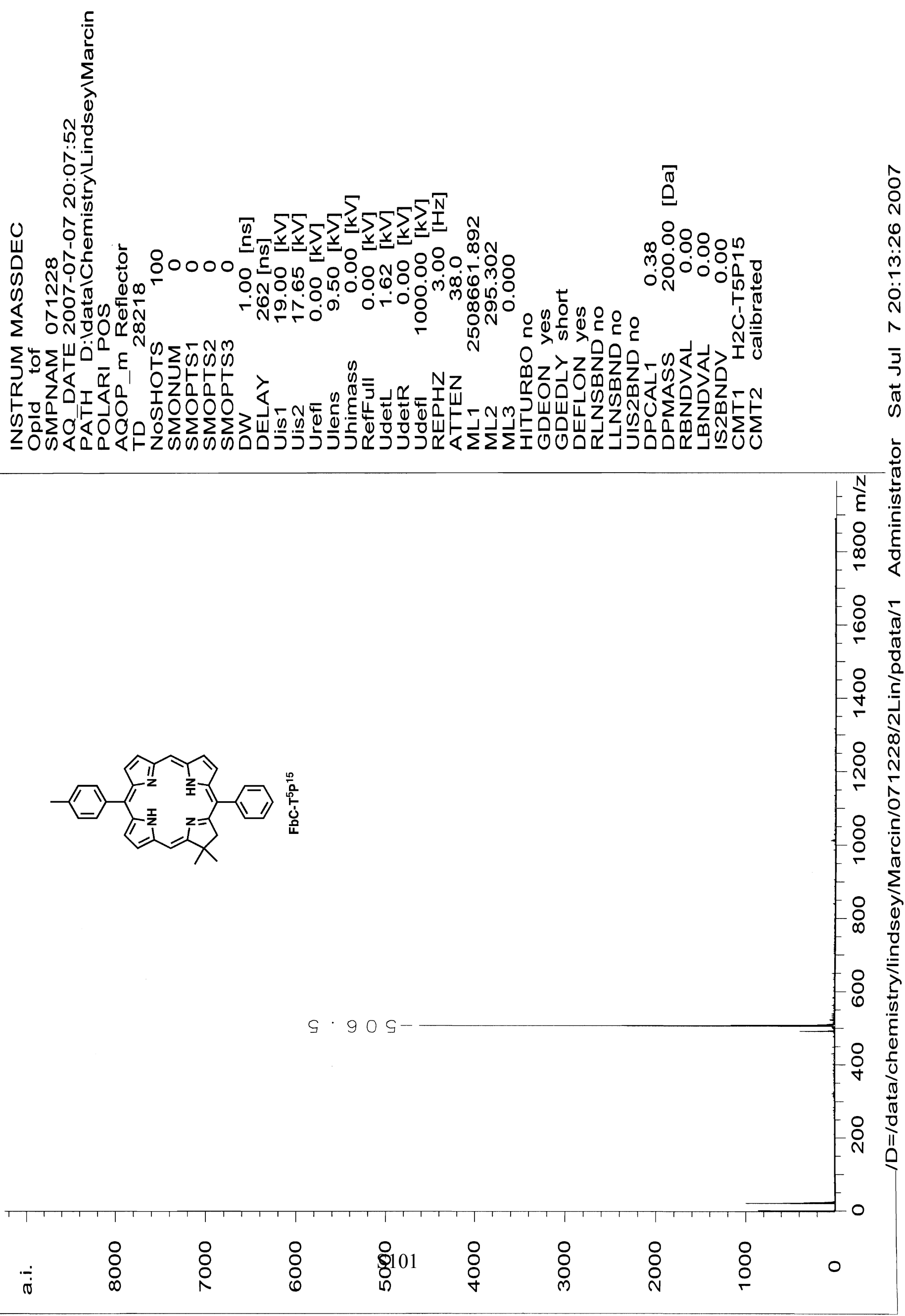




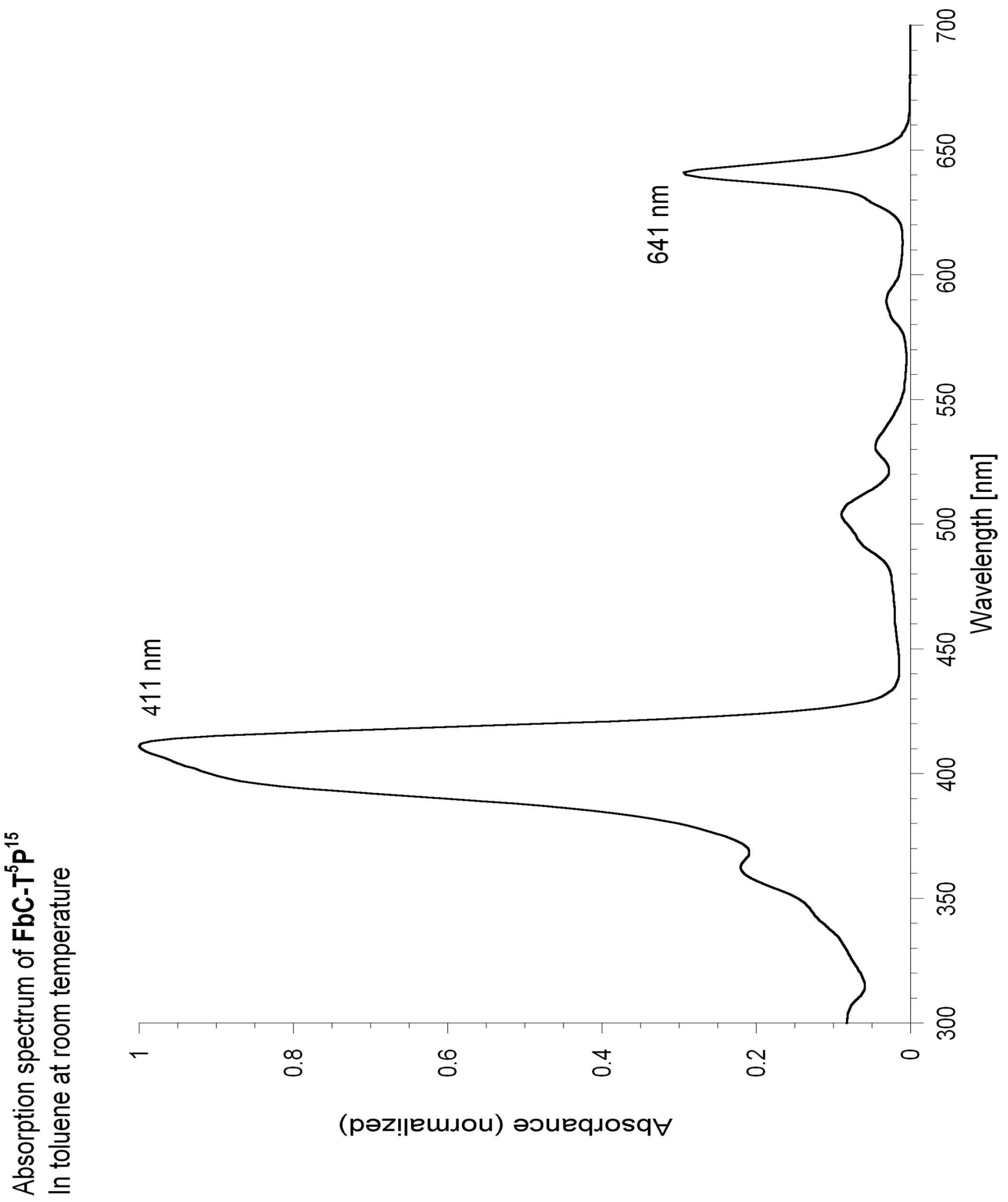




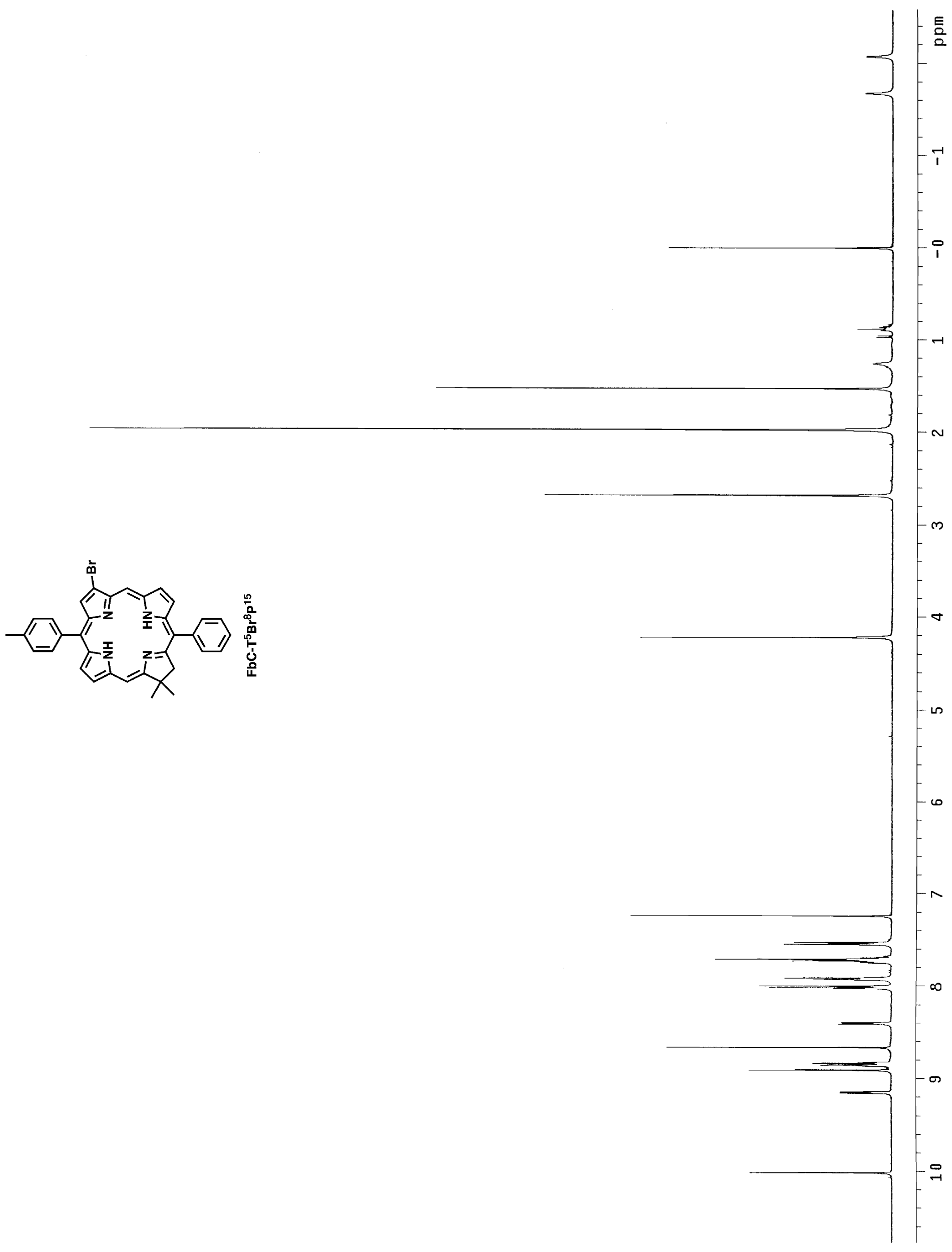




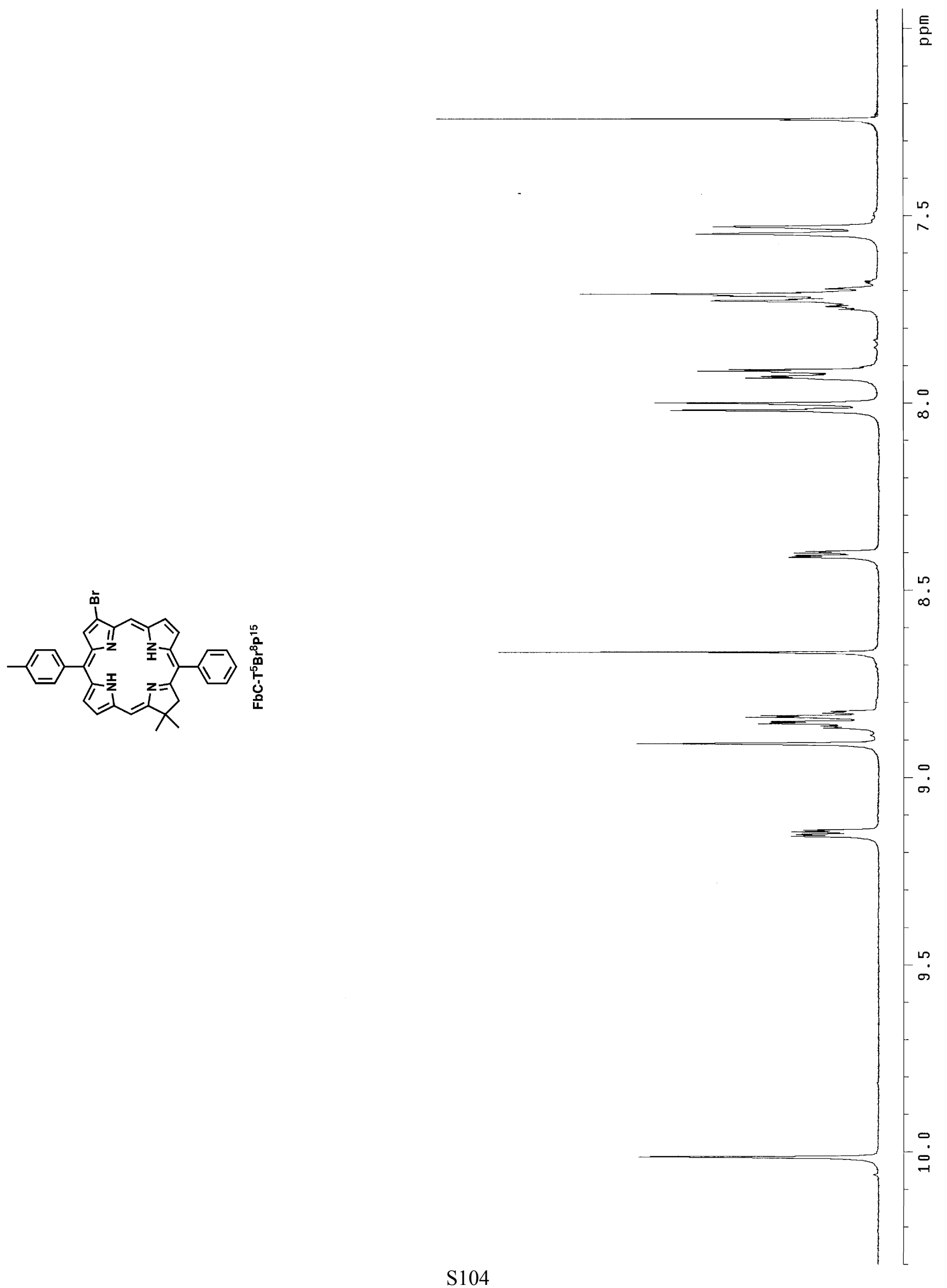




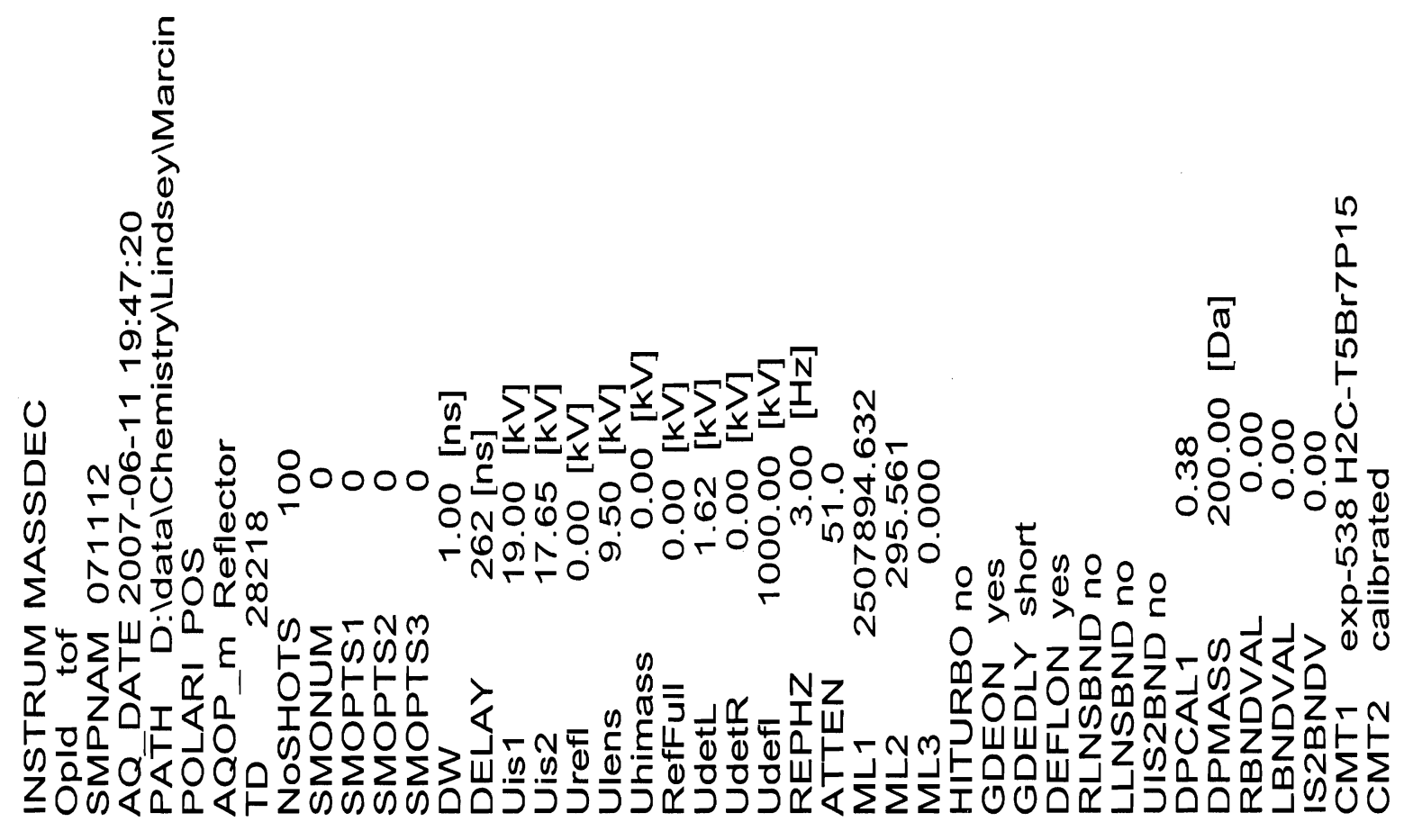

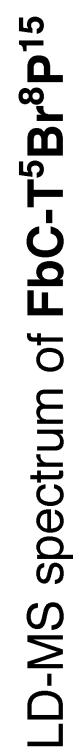

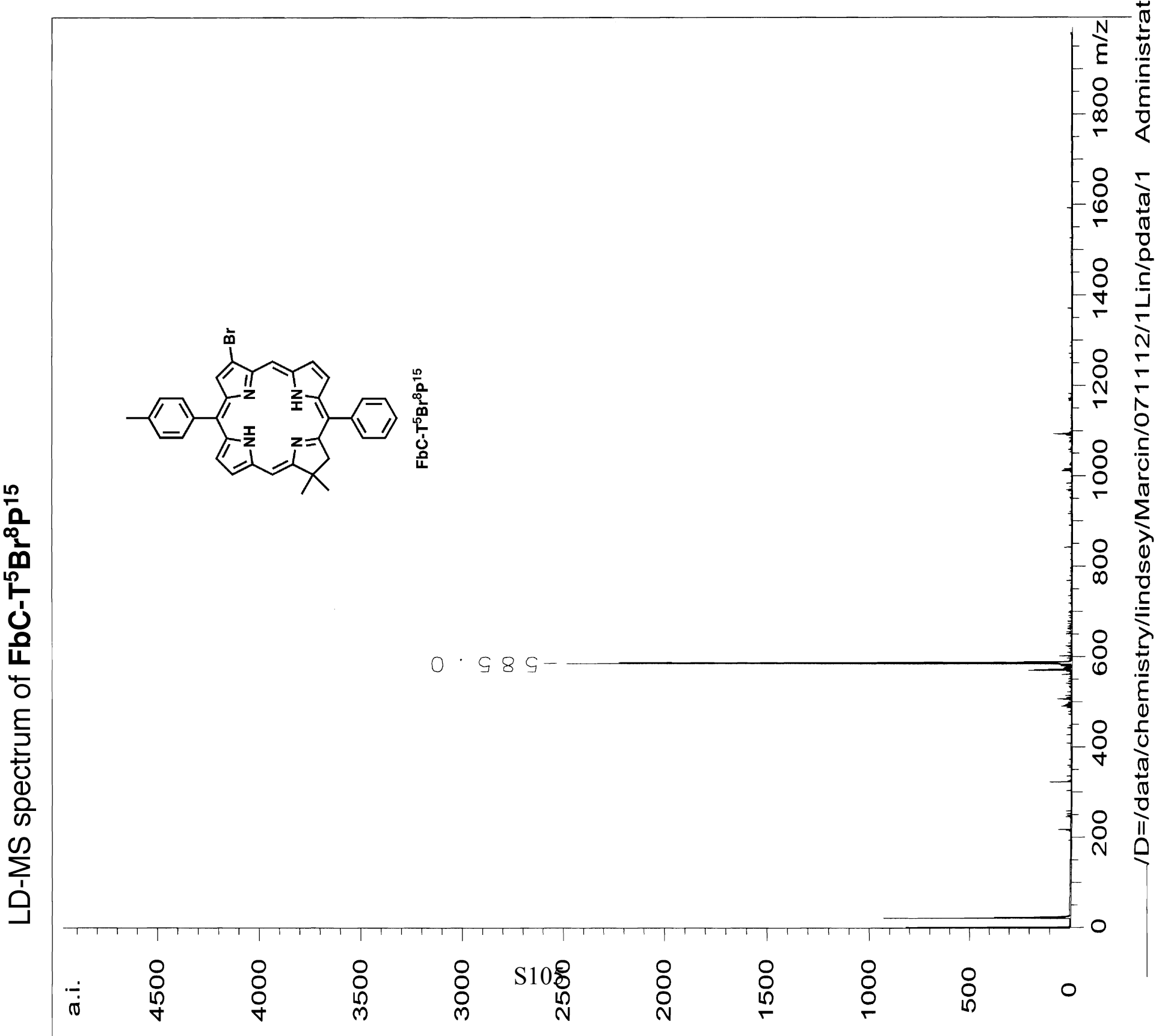




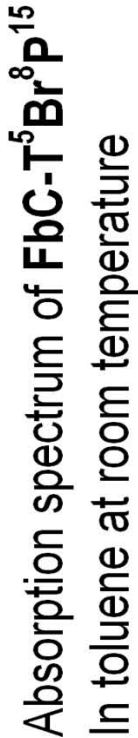

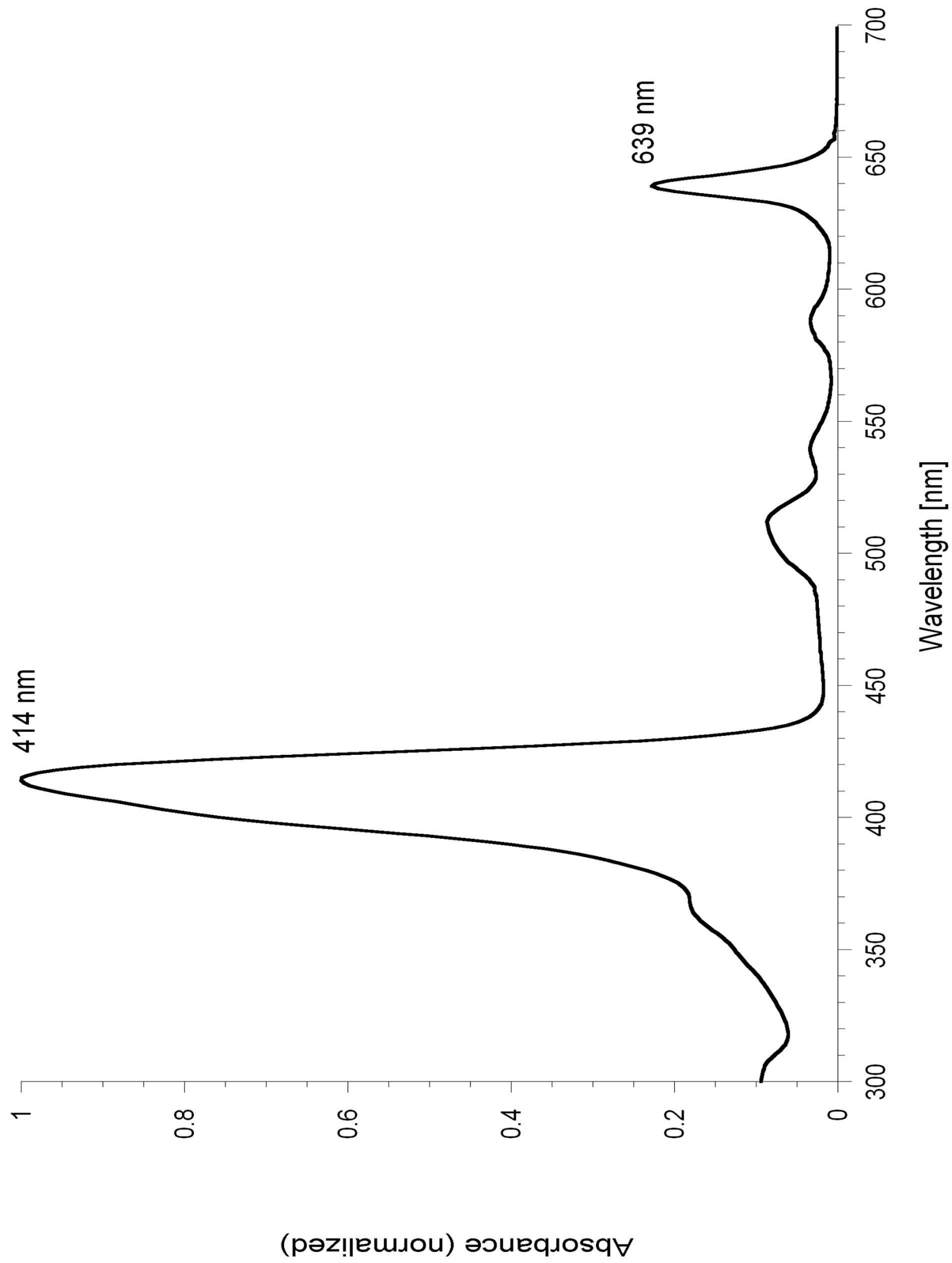




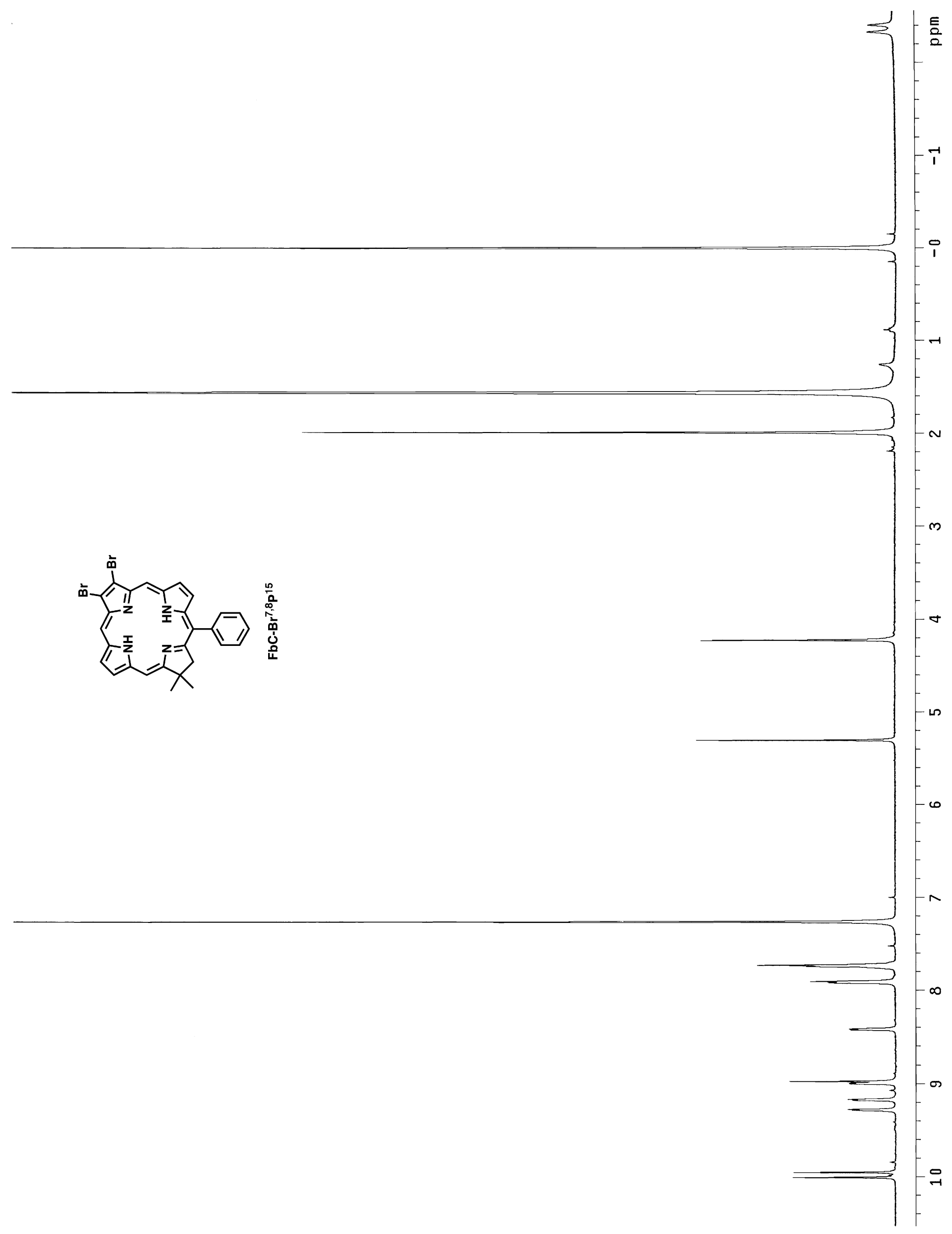




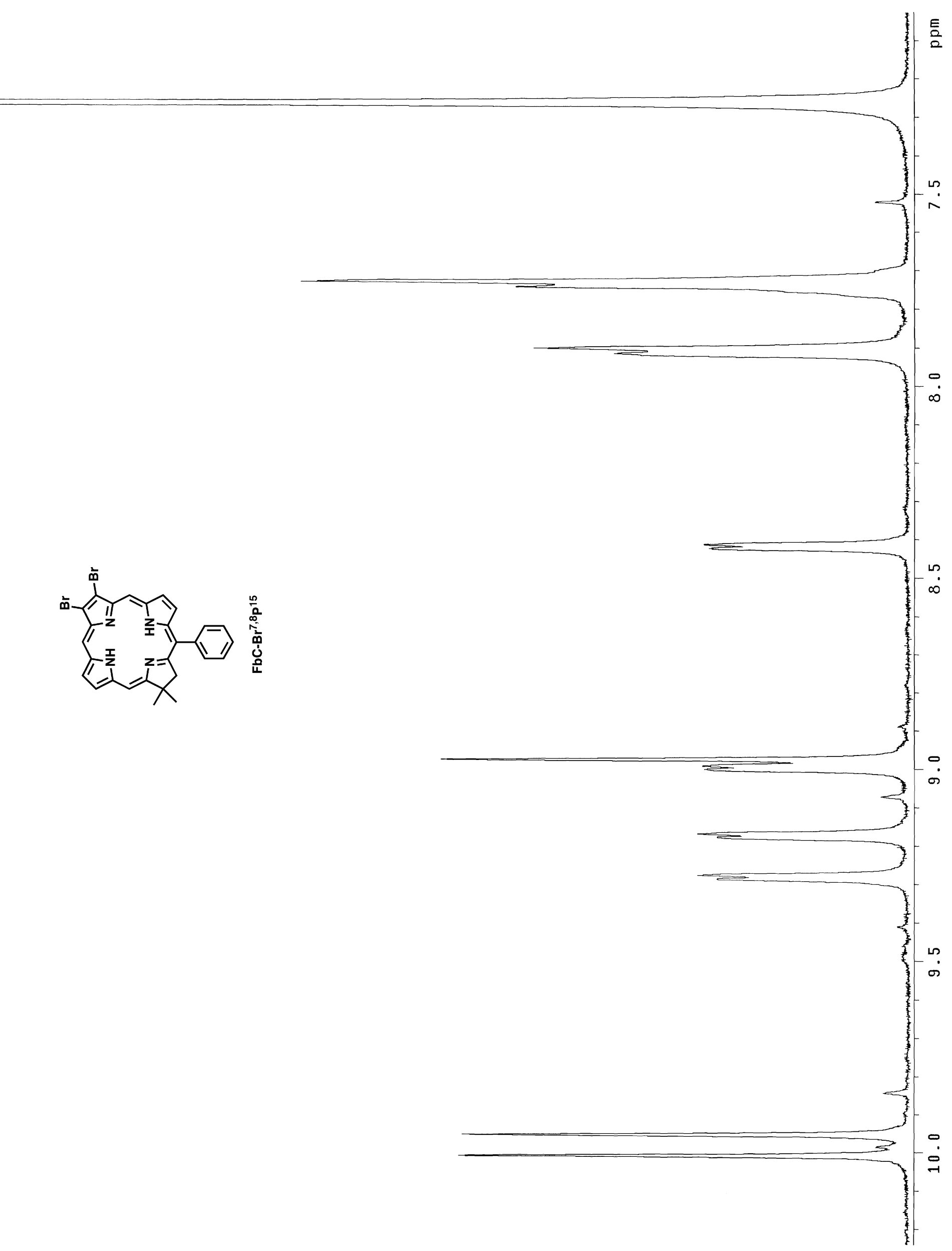




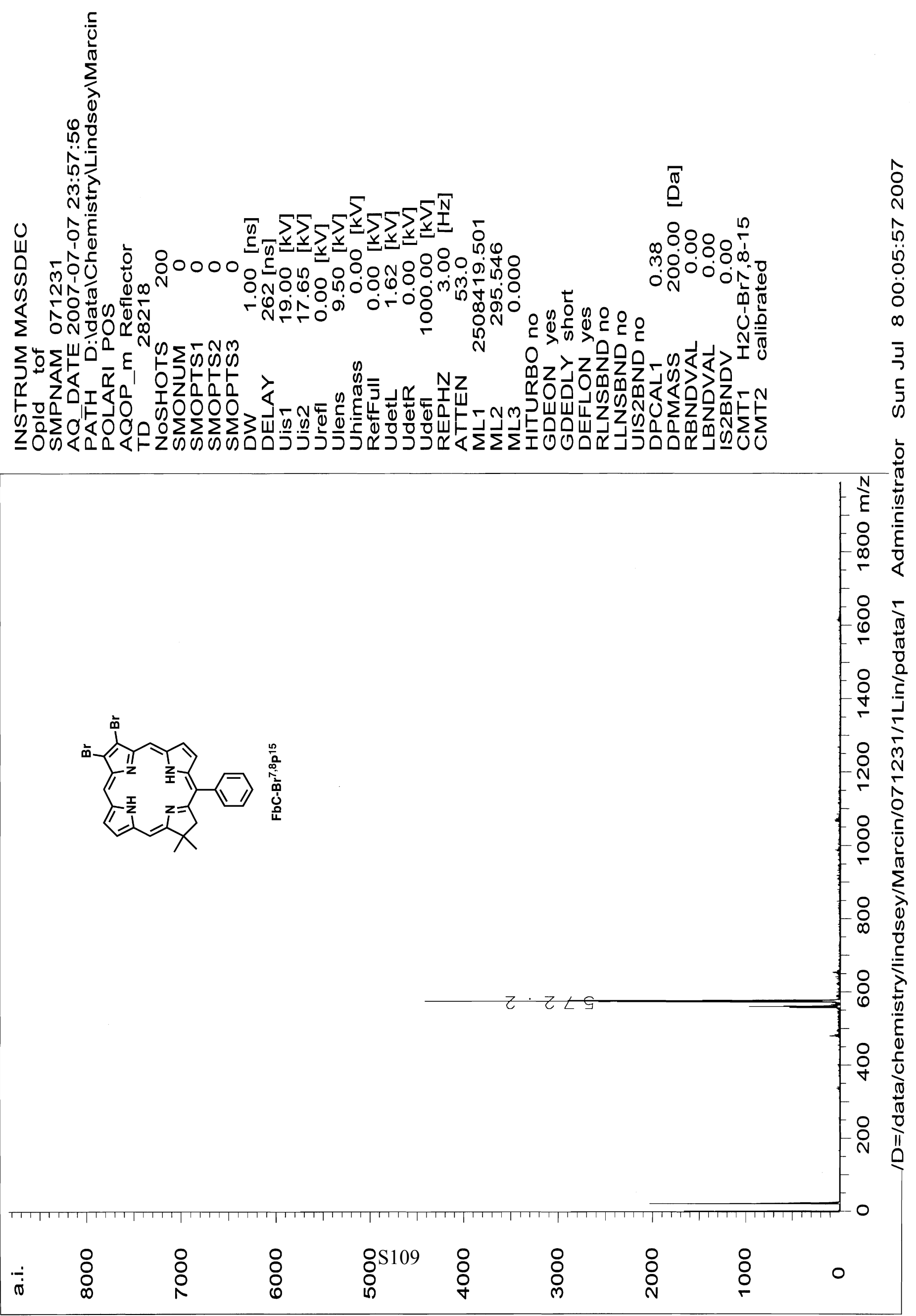




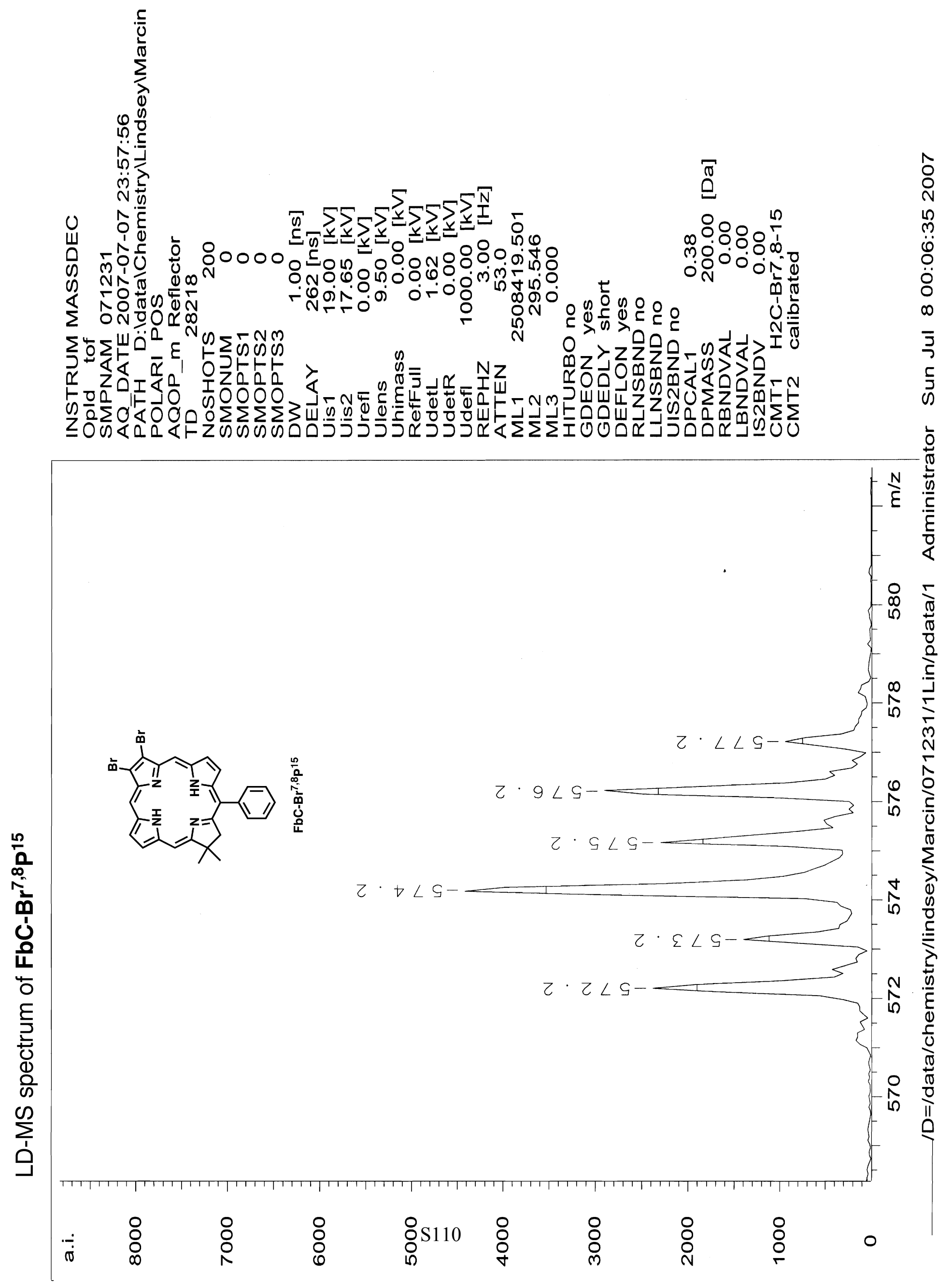



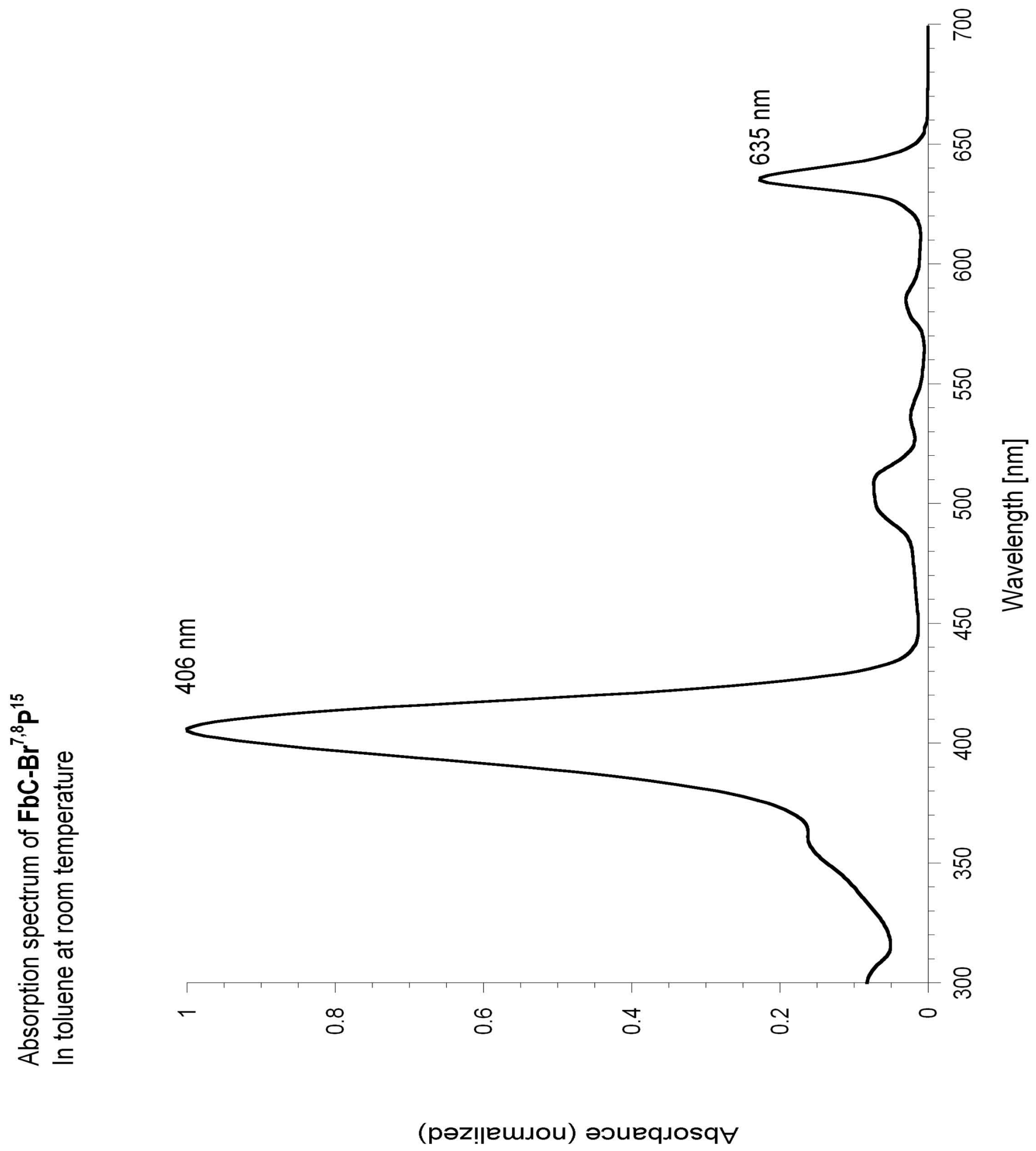